\title{
On the Utility of Genomics-Based Methods for Surveillance of Antimicrobial-Resistant Bacteria in the Food Production Continuum
}

\author{
by
}

\section{Ashley Cooper}

A thesis submitted to the Faculty of Graduate and Postdoctoral Affairs in partial fulfillment of the requirements for the degree of

\author{
Doctor of Philosophy \\ in
}

Biology

Carleton University

Ottawa, Ontario

(C) 2020

Ashley Cooper 


\section{Abstract}

Overuse of antimicrobials in medicine and agriculture are believed to be drivers of the spread of AMR among pathogenic bacteria. Antimicrobial use in agriculture and food-producing animals may facilitate spread of resistant bacteria to food products as well as dissemination into the environment. Although regulatory practices currently monitor food, feed, and fertilizer products for presence of pathogenic organisms, they do not test for AMR genes (ARGs).

My $\mathrm{Ph} . \mathrm{D}$. thesis focuses on filling current knowledge gaps for transmission and detection of AMR in food production by evaluating methodology to monitor transmission of resistance genes throughout the food production continuum. There are three main objectives of my thesis: (i) to evaluate the accuracy of genotype in predicting resistance phenotypes; (ii) to investigate the presence and mechanisms of resistance in food production; (iii) to determine whether short-read metagenomics is a suitable highthroughput method for surveillance of resistance in agri-food products. For the first objective, concordance of AMR phenotypes with whole genome sequencing (WGS) predictions of ARGs were determined in two separate studies. Requirements for sequence coverage suggest genotypic predictions of AMR are highly concordant when using a target-gene identity cutoff of $>80 \%$, assuming sufficient genome coverage.

To investigate pathogen persistence in food production, analyses were conducted using WGS data for 1279 L. monocytogenes isolated from food products. The most frequently isolated clonal complexes were significantly associated with carriage of plasmid-borne quaternary ammonium compound resistance. Additionally, conjugation 
frequencies of plasmid-borne AMR between commensal food bacteria and a foodborne pathogen were determined. High rates of both pathogen persistence and plasmid transfer in foodborne bacteria emphasize the importance of monitoring resistance determinants in commensal food bacteria as well as pathogens.

Finally, to determine the utility of shotgun metagenomics for monitoring AMR in agri-food production I conducted a study on LOD of ARGs in metagenomic sequences. This study highlights that as the number of sequence reads decreases, target organisms must comprise a larger proportion for ARG detection.

This research emphasizes the importance of monitoring resistance dissemination throughout agri-food production and has provided guidance to aid in selection of appropriate methods for surveillance of AMR in food products. 


\section{Acknowledgements}

There are many people to thank for their support. First, I would like to express gratitude and thanks to my supervisors at the Canadian Food Inspection Agency, Dr. Burton Blais and Dr. Catherine Carrillo for all of their guidance and support. Thank you for providing me this opportunity to pursue research. Thank you to Dr. Alex Wong who was always available and happy to answer questions and provided guidance on many of my statistical analyses. I am privileged to have worked with and learned from such excellent scientists.

I would like to thank my committee members, Dr. Alexandre Poulain and Dr. John Vierula who provided excellent advice on my research. I would also like to thank Dr. Sandeep Tamber for her assistance and collaboration with experiments and manuscript preparation.

In addition, thank you for all members of the OLC-CFIA and Wong labs for their collaboration, comments and feedback on my work. Thank you to the CFIA-OLC bioinformatics team, both past and present, for teaching and encouraging me to complete bioinformatic analyses myself.

Last but not least, thank you to my family - who have always been supportive of any and all of my ventures even though they may not always understand what it is I am doing. To my parents, for all of their blessings and for helping to shape me into the person I have become. My little brother Cory, for allowing me to explain my work to him (even though he never asked). Also, to my cat Gort for acting as emotional support during writing, while also attempting to add a few words herself (unfortunately I have been informed that listing your cat as a co-author is not as accepted in biology as it is in physics). And thank you to my partner Paul, for always being supportive, and for offering to review all of my work. Thank you for everything. 


\section{Statement of Contribution}

The thesis, "On the Utility of Genomics-Based Methods for Surveillance of Antimicrobial-Resistant Bacteria in the Food Production Continuum" is composed of five studies.

Chapter 2, "Systematic Evaluation of Whole Genome Sequence-Based Predictions of Salmonella Serotype and Antimicrobial Resistance", is the result of experiments designed by myself and Dr. Catherine Carrillo with contributions from Dr. Burton Blais. Experiments and data compilation were carried out by myself, with bioinformatics assistance from the team at the Canadian Food Inspection Agency Ottawa Laboratory Carling. Dr. Catherine Carrillo contributed to the conceptualization of experiments and drafting of the manuscript. Dr. Carrillo, Dr. Blais, and Dr. Wong contributed to editing of the manuscript. A version of this chapter has been published in Frontiers in Microbiology.

Chapter 3, "Detection of carbapenem-resistance genes in bacteria isolated from wastewater in Ontario", is the result of experiments primarily designed and carried out by myself in collaboration with students from the Algonquin College Biotechnology Advanced program, with guidance from Dr. Burton Blais and Dr. Catherine Carrillo. The students at Algonquin College: Cassandra Carter, Hana McLeod, Marie Wright, and Prithika Sritharan; isolated bacteria from samples I coordinated and conducted PCR analyses. Experimental analysis of bacterial isolates including PCR, DNA extractions, whole-genome sequencing, and computational analysis was completed by myself. Species identification was conducted using Matrix-assisted laser desorption/ionization at the Health Canada Bureau of Microbial Hazards with the collaboration of Dr. Sandeep Tamber. The manuscript was prepared and edited by myself, Dr. Wong, Dr. Carrillo, Dr. Blais, and Dr. Tamber. A version of this chapter has been accepted for publication in FACETS.

Chapter 4, "Genomic Markers for Quaternary Ammonium Compound Resistance as a Persistence Indicator for Listeria monocytogenes Contamination in Food Manufacturing Environments", is the result of experiments designed and conceptualized by Dr. Burton Blais. In vitro growth assays were conducted by Mylène Deschênes. Sequence data analysis and curation was conducted by Dr. Catherine Carrillo and myself, SNV-analysis was conducted by Dr. Carrillo. Computational and statistical analyses were conducted by myself. Manuscript was prepared by Dr. Blais, Dr. Carrillo, and myself. A version of this 
chapter has been published in the Journal of Food Protection with Dr. Carrillo as coauthor.

Chapter 5, "Foodborne Bacteria Facilitate Transmission and Persistence of Clinically Important Antimicrobial Resistance", is the result of experiments designed and carried out by myself. Dr. Burton Blais, Dr. Catherine Carrillo, Dr. Alex Wong, and Dr. Sandeep Tamber provided guidance for data analysis.

Chapter 6, "Modeling the Limits of Detection of Antimicrobial Resistance Genes in Agri-Food Metagenomic Samples", is the result of experiments designed and carried out by myself and Dr. Catherine Carrillo. Bioinformatic tools for metagenome synthesis were created by Andrew Low. Isolate selection, metagenome synthesis, data analysis, and writing were completed by myself. Dr. Carrillo, Dr. Blais, and Dr. Wong contributed to drafting and editing. A version of this chapter was presented in scientific poster format at the American Society for Microbiology Conference on Rapid Applied Microbial NextGeneration Sequencing and Bioinformatic Pipelines in December 2020. 


\section{Table of Contents}

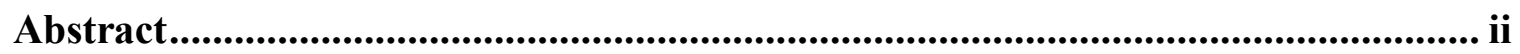

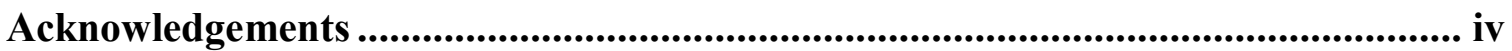

Statement of Contribution..........................................................................................

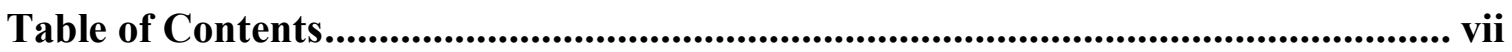

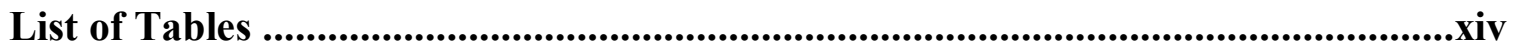

List of Illustrations ................................................................................................... xvii

List of Appendices ...............................................................................................................xix

List of Abbreviations .........................................................................................................

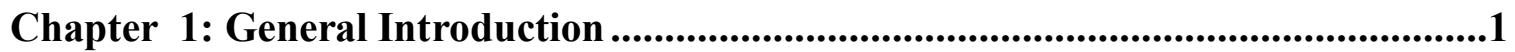

1.1 Antimicrobials and antimicrobial resistance (AMR) …..................................... 1

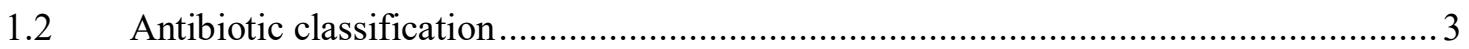

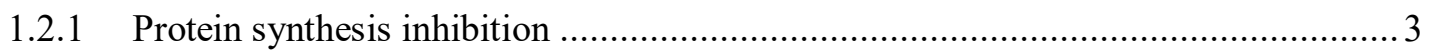

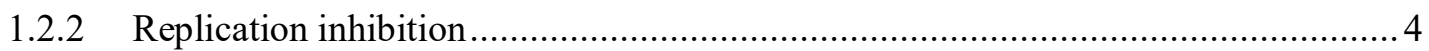

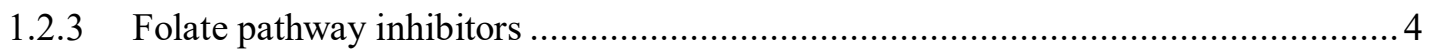

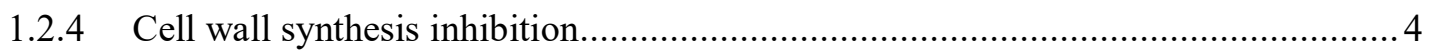

1.3 Antimicrobial classification according to importance in human medicine.................. 6

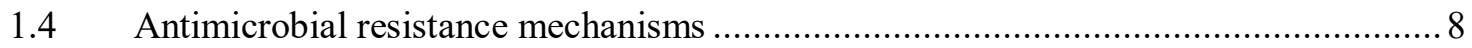

1.4.1 Diminishing access to antimicrobial target via influx/efflux modification ............... 8

1.4.2 Modification of antimicrobial target(s) .....................................................

1.4.3 Modification or inactivation of the antimicrobial ........................................... 10

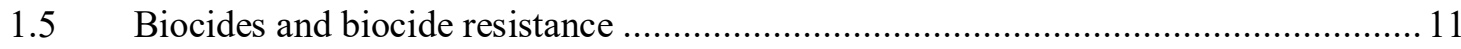

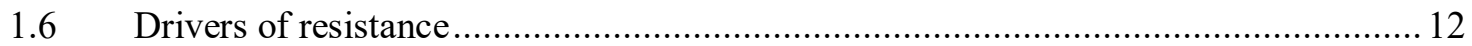

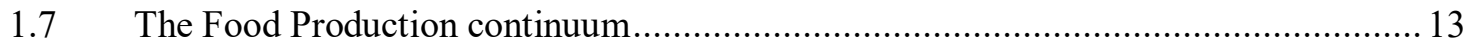


1.8 Filling the gaps in agri-food testing and resistance surveillance.

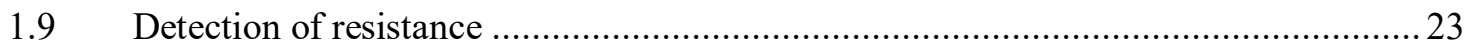

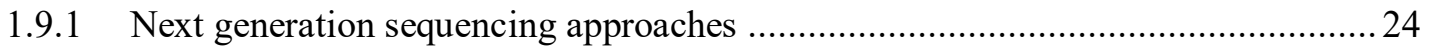

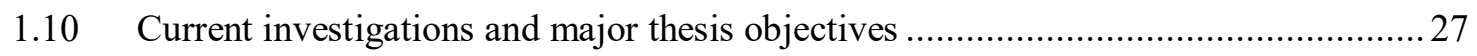

\section{Chapter 2: Systematic Evaluation of Whole Genome Sequence-Based Predictions of Salmonella Serotype and Antimicrobial Resistance .................................................31}

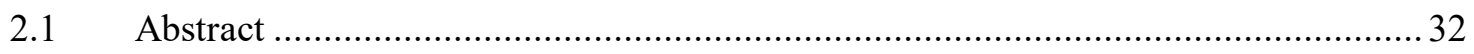

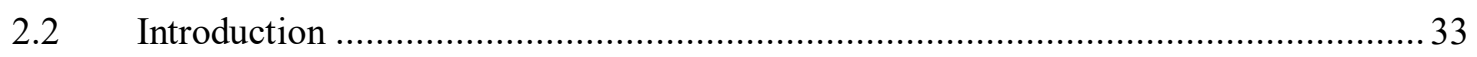

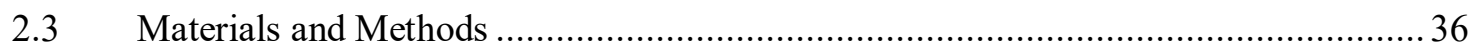

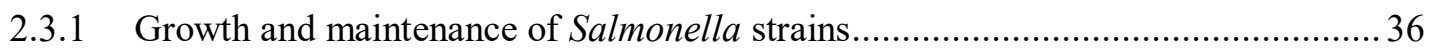

2.3.2 Traditional Serotyping and Antimicrobial susceptibility testing .......................... 37

2.3.3 gDNA isolation and whole-genome sequencing (WGS) of selected Salmonella .... 38

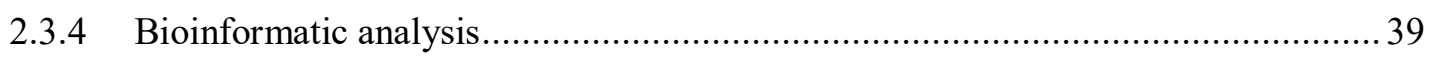

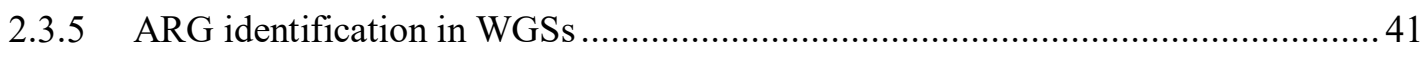

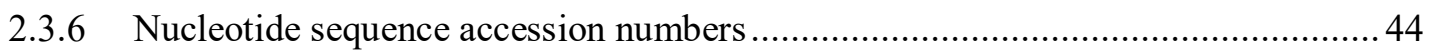

2.3.7 Raw read sampling to determine minimum coverage requirements for ARG

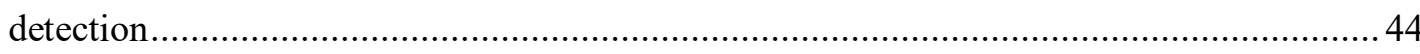

2.3.8 Analysis of ARG-detection in assembled versus raw-read sequences ................... 45

2.3.9 Resistance phenotype verification via broth microdilution ................................ 46

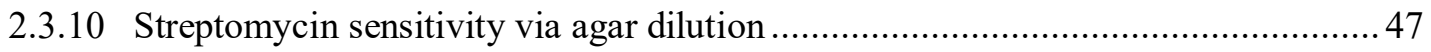

2.3.11 Activation of Cryptic Aminoglycoside Resistance in Minimal Media .................. 47

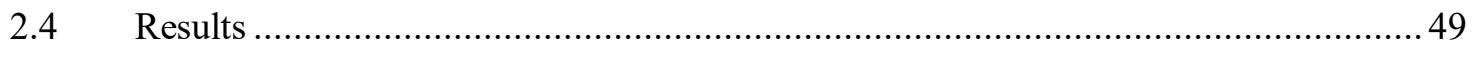

2.4.1 Determining Salmonella spp. Serotypes in silico ............................................ 49

2.4.2 Antimicrobial Resistance: Relationship of Phenotype and Genotype .................... 50

2.4.3 Minimum Coverage Requirements for Accurate ARG Determination ................... 55

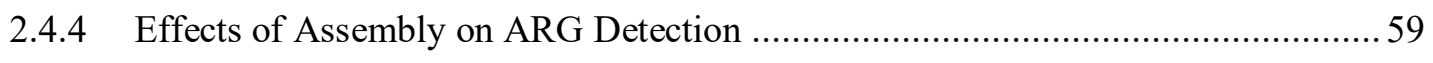


2.4.5 Single Nucleotide Polymorphisms (SNPs) Conferring AMR ..............................62 62

2.4.6 Minimal media induces cryptic aadA in Salmonella spp. isolates .......................... 64

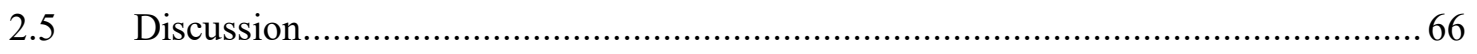

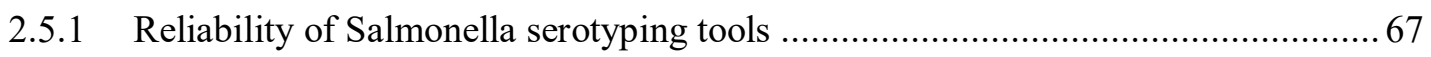

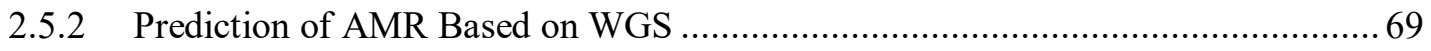

2.5.3 Requirements for WGS-Based ARG Detection.............................................. 70

2.5.4 Single nucleotide variations (SNVs) conferring AMR ….................................. 72

2.5.5 Activation of STR Resistance in Salmonella spp. Occurs in Minimal Media ......... 76

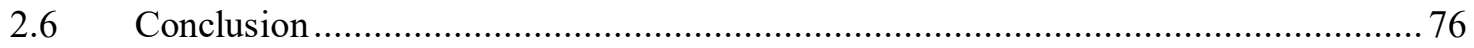

\section{Chapter 3: Detection of carbapenem-resistant genes in bacteria isolated from} wastewater in Ontario ..............................................................................................78

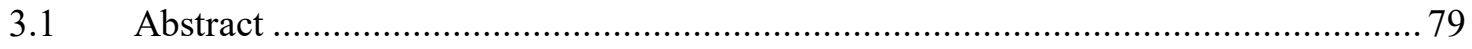

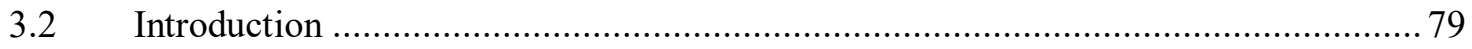

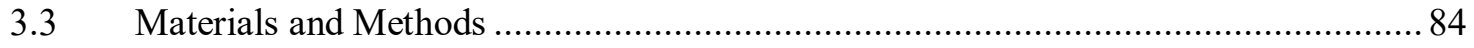

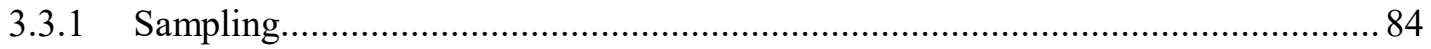

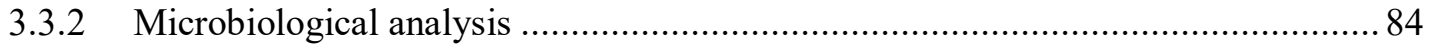

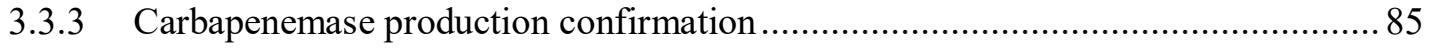

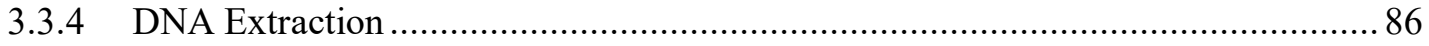

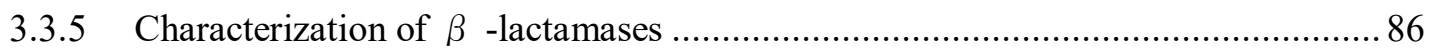

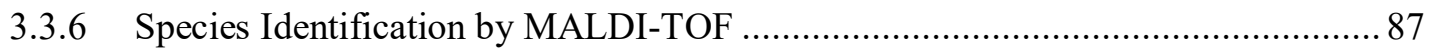

3.3.7 Whole Genome Sequencing (WGS) and in silico ARG Detection ......................... 88

3.3.8 Nucleotide sequence accession numbers ................................................... 90

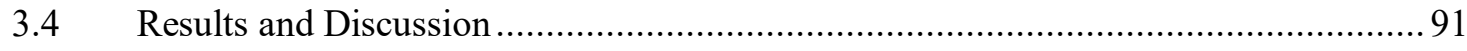

3.4.1 Characterization of carbapenem-resistant strains recovered from WWTP influents91

3.4.2 Identification of Antibiotic Resistance Genes (ARGs) in Isolates found in WWTP

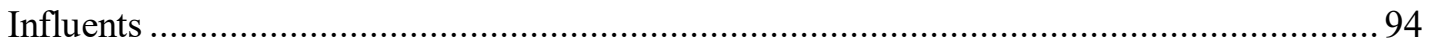




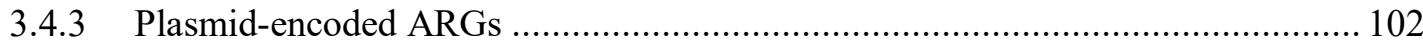

3.4.4 Use of indicator organisms for monitoring AMR in WWTPs ............................. 103

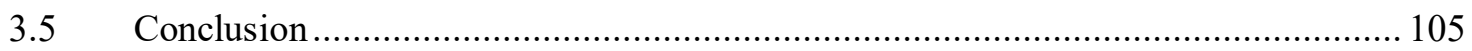

\section{Chapter 4: Genomic Markers for Quaternary Ammonium Compound Resistance as a Persistence Indicator for Listeria monocytogenes Contamination in Food} Manufacturing Environments........................................................................108

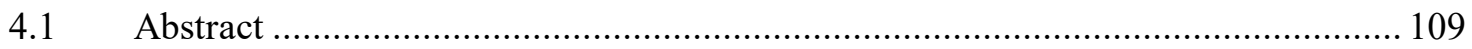

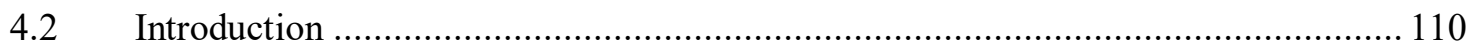

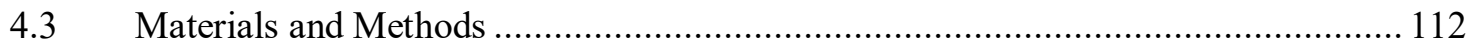

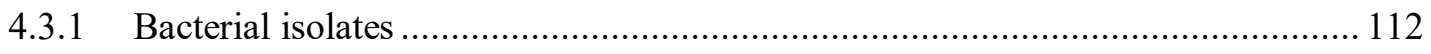

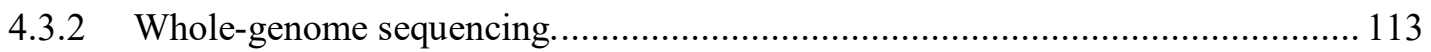

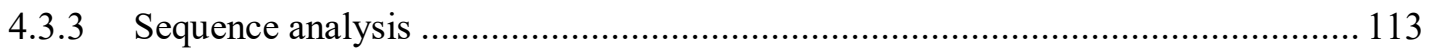

4.3.4 Antimicrobial and biocide resistance gene detection in silico ............................ 114

4.3.5 Identification and removal of duplicate isolates .......................................... 115

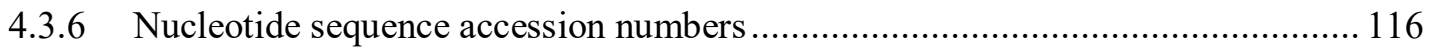

4.3.7 Multiplex $b c r A, b c r B$ and $b c r C$ Polymerase chain reaction (PCR) ...................... 116

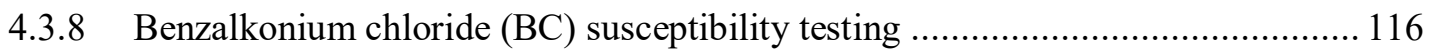

4.3.9 Statistical tests to identify associations of QAC-resistance with food sources and

CCs 117

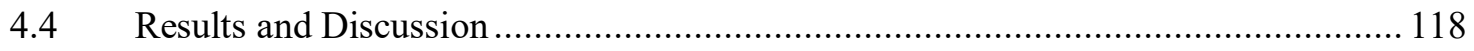

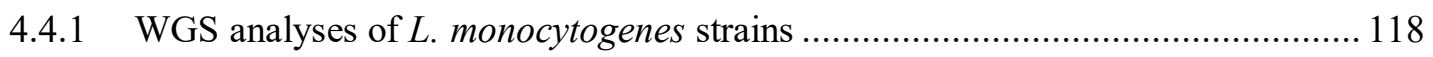

4.4.2 Correlation of expressed resistance with occurrence of the bcrABC cassette ....... 122

4.4.3 Determination of $b c r A, b c r B$ and $b c r C$ by polymerase chain reaction ................ 125

4.4.4 Stability of plasmid-encoded bcr $A B C$ in foodborne L. monocytogenes isolates ... 125

4.4.5 Carriage of QAC-resistance in clinically-relevant clonal complexes .................. 127

4.5 Implementation of testing for sanitizer resistance in food testing programs ............ 129 


\section{Chapter 5: Foodborne Bacteria Facilitate Transmission and Persistence of Clinically Important Antimicrobial Resistance....................................................131}

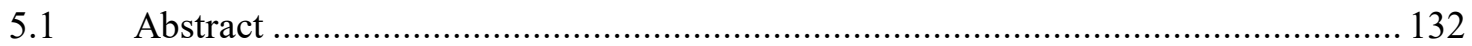

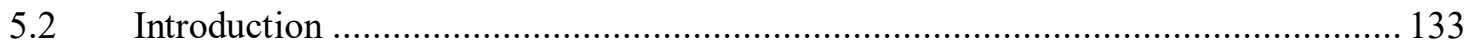

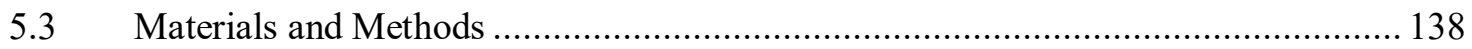

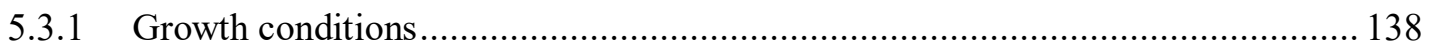

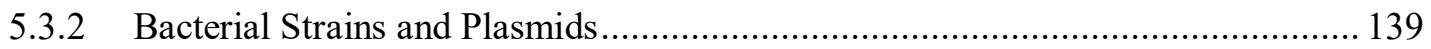

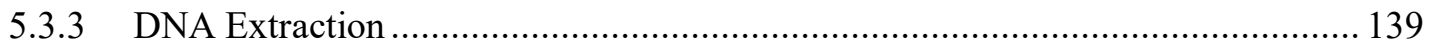

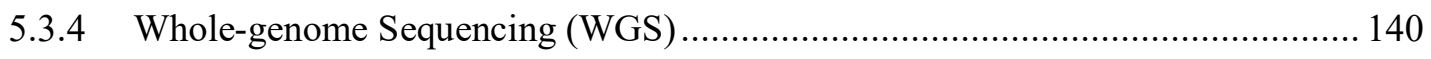

5.3.5 WGS Screening of Candidate Recipient Strains for AMR and Plasmids.............. 140

5.3.6 Creation of rifampicin-resistant mutants for conjugation experiments ................ 143

5.3.7 Evaluation of RifR Retention and Fitness ................................................... 143

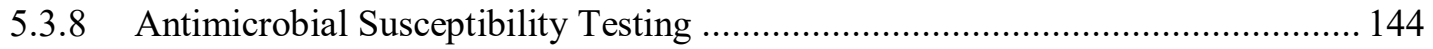

5.3.9 Multiplex PCR (mPCR) for Plasmid Recipient Verification............................... 144

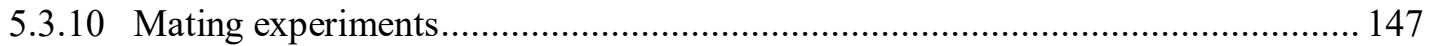

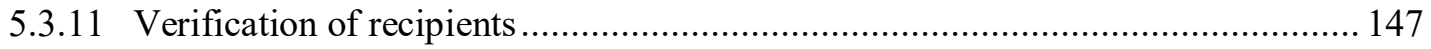

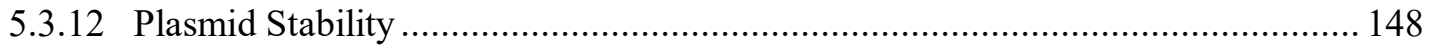

5.3.13 Phylogenetic tree construction from bacterial core genomes ............................. 149

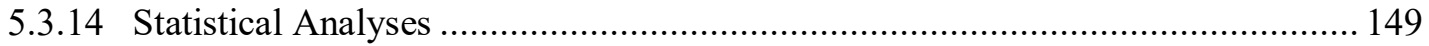

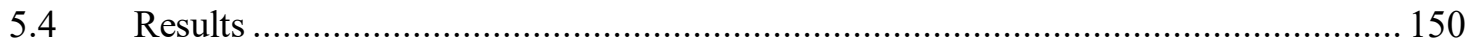

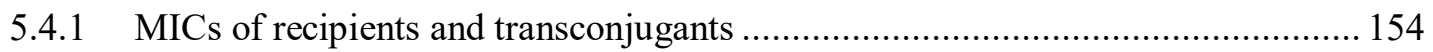

5.4.2 Impact of CTX concentration on enumeration of transconjugants ...................... 156

5.4.3 Impact of Nutrients and temperature on conjugation frequency ............................. 156

5.4.4 Between strains comparison of conjugation frequency .................................. 157

5.4.5 Transfer rate as a function of donor-recipient relatedness................................ 160

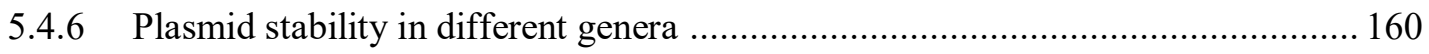




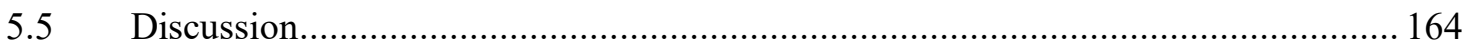

5.5.1 Production of various morphotypes by Hafnia alvei recipients ........................... 164

5.5.2 Environmental conditions had minimal effect on conjugation frequency to

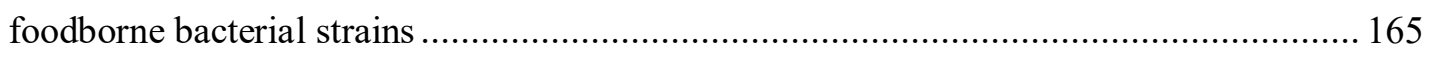

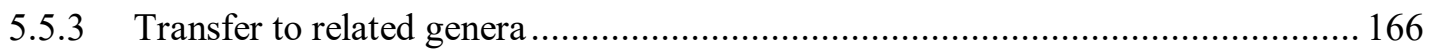

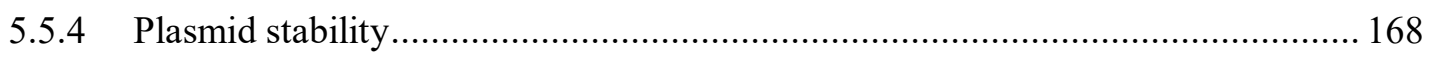

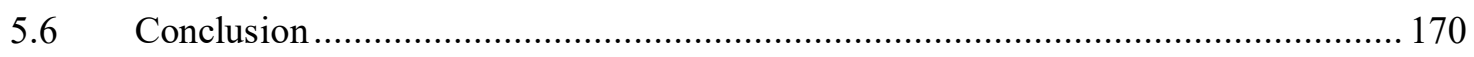

\section{Chapter 6: Modeling the Limits of Detection of Antimicrobial Resistance Genes in} Agri-Food Metagenomic Samples ......................................................................171

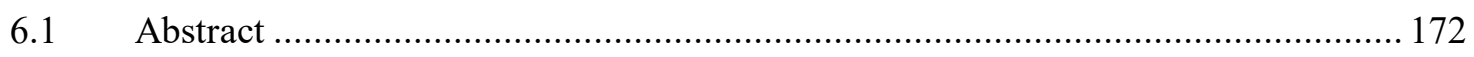

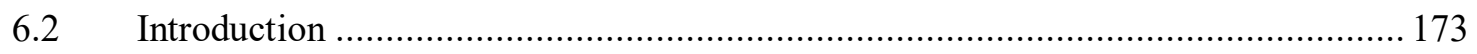

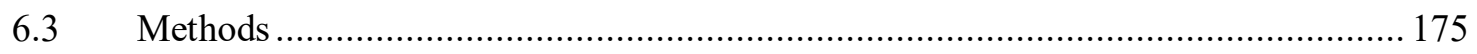

6.3.1 Sequences Used in Mock Metagenome Synthesis ....................................... 175

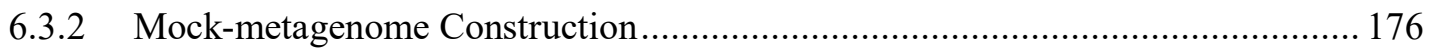

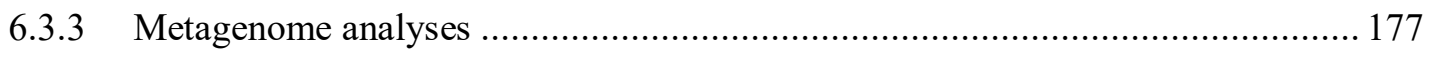

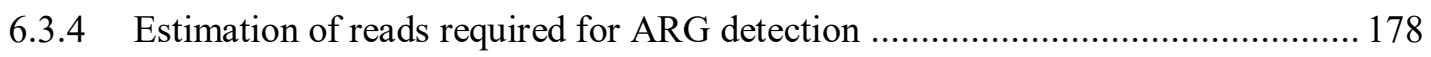

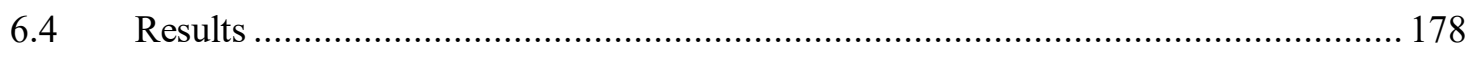

6.4.1 Coverage of organism affects ARG detection in metagenomes ........................ 178

6.4.2 Proportion of Isolate Reads in Metagenome Required for ARG Detection .......... 183

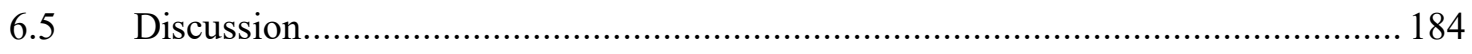

Chapter 7: Concluding Remarks ...............................................................................188

Chapter 8: Future Perspectives................................................................................193

Appendices ..........................................................................................................................199

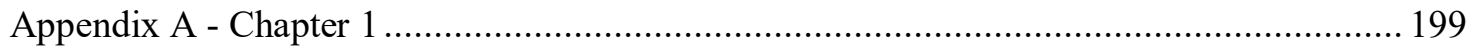

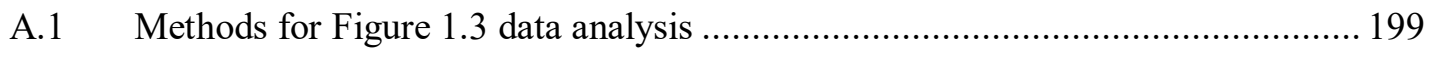

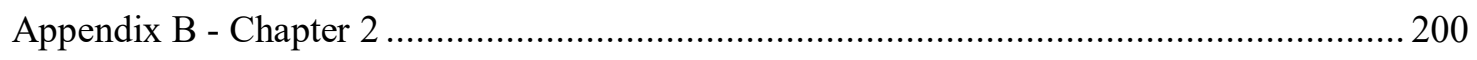




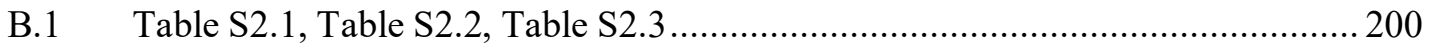

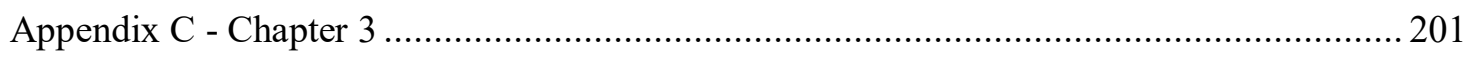

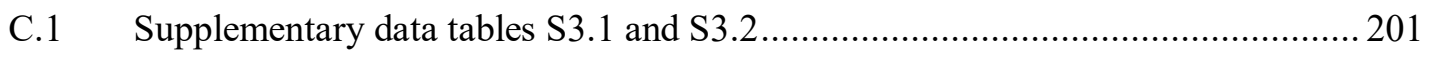

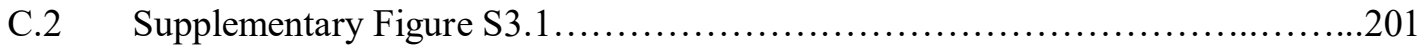

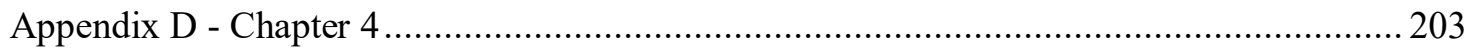

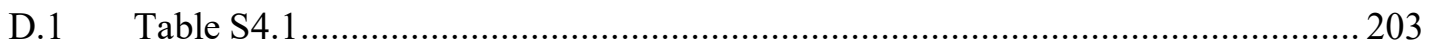

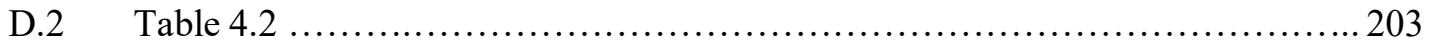

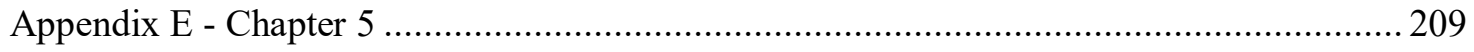

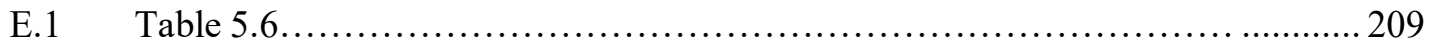

References .....................................................................................................210 


\section{List of Tables}

TABLE 1.1. ANTIMICROBIAL TARGETS AND MECHANISMS OF ACTION, AND BACTERIAL MECHANISMS OF RESISTANCE TO COMMON ANTIMICROBIALS................................5

TABLE 1.2. HEALTH CANADA AND WHO CATEGORIZATION OF ANTIMICROBIAL CLASSES ......7

TABLE 2.1. CHARACTERISTICS OF ARG DETECTION TOOLS. ...........................................42

TABLE 2.2. IDENTIFICATION OF SINGLE NUCLEOTIDE VARIATIONS RESULTING IN NON-

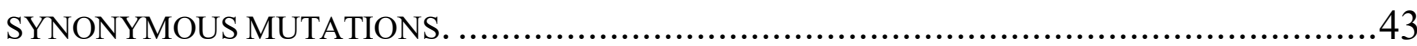

TABLE 2.3. ARG PROFILES AND LOCATIONS IN SUBSET OF S. ENTERICA SUBSPECIES ENTERICA ISOLATES USED FOR ASSEMBLY VERSUS RAW-READ ANALYSES. ............................45

TABLE 2.4. PERFORMANCE OF IN SILICO TOOLS FOR DETECTING SALMONELLA SEROTYPE ....50

TABLE 2.5. PREDICTING SALMONELLA SEROTYPES USING WGS DATA .52

TABLE 2.6. ACCURACY OF AMR PHENOTYPE PREDICTIONS IN SALMONELLA BY AMR-GENE PREDICTION TOOLS

TABLE 2.7. BROTH MICRODILUTION TESTING OF ISOLATES WITH GENOTYPE-PHENOTYPE DISCREPANCIES .56

TABLE 2.8. STR MINIMUM INHIBITORY CONCENTRATION OF SALMONELLA ISOLATES IN MH, LB, AND M9 AGAR AND BROTH

TABLE 3.1. PRIMERS USED FOR B-LACTAMASE, ESBL, AND CARBAPENEMASE GENE

DETECTION .88

TABLE 3.2. NUMBER OF ISOLATES CONFIRMED BY MODIFIED HODGE TEST (MHT) TO BE CARBAPENEMASE PRODUCERS 
TABLE 3.3. SUMMARY OF MOLECULAR AND FUNCTIONAL CHARACTERISTICS OF B-

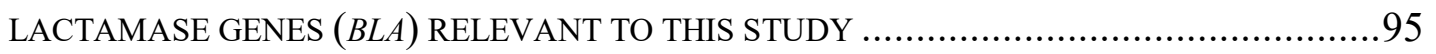

TABLE 4.1. LISTERIA MONOCYTOGENES BCRABC PROFILES BY CLONAL COMPLEX AND SOURCE

TABLE 4.3. Clusters OF L. MONOCYTOGENES WITH VARIABLE PRESENCE OF BCRABC AMONG ISOLATES

TABLE 5.1. BACTERIAL GENERA, SOURCE(S) AND LOCATION(S) WHERE BLA $A_{\mathrm{CMY}-2}$ HAS BEEN REPORTED 136

TABLE 5.2. BACTERIAL DONOR STRAIN, PLASMID TYPES, AND ANTIMICROBIAL RESISTANCE GENES 139

TABLE 5.3. BACTERIAL DONOR STRAIN, PLASMID TYPES, AND ANTIMICROBIAL RESISTANCE GENE(S) 141

TABLE 5.4. PRIMERS USED FOR TRANSCONJUGANT CONFIRMATION 146

TABLE 5.5. MINIMUM INHIBITORY CONCENTRATIONS (MIC) OF CEFOTAXIME (CTX) FOR RECIPIENTS AND TRANSCONJUGANTS ON MHA, UCHROM, AND LBA-LENNOX. 155

TABLE 6.1. CHARACTERISTICS OF SEQUENCES USED FOR MOCK-METAGENOME SYNTHESIS

TABLE 6.2. REQUIRED ISOLATE ABUNDANCE (\%) IN METAGENOMES OF VARYING SIZES FOR DETECTION OF ANTIMICROBIAL RESISTANCE GENES IN ISOLATES WITH 3, 4, OR 5 MBP GENOMES. 178

TABLE 6.3. NuMBER OF CMY-GENE(S) AND ALLELE(S) DETECTED BY KMA AND SRST2 IN BEEF METAGENOMES CONTAINING S. SER. HEIDELBERG ISOLATE PRESENT AT 1X GENOME COVERAGE. 
APPENDIX D.1 - TABLE 4.2. GENETIC PROFILES OF L. MONOCYTOGENES USED IN PHENOTYPIC RESISTANCE

STUDIES..........................................................204

APPENDIX E.1 - TABLE 5.6. PAIRWISE PHYLOGENETIC DISTANCE OF STRAIN RELATEDNESS

COMPARED TO DONOR SALMONELLA ENTERICA SER. HEIDELBERG DONOR.................209 


\section{List of Illustrations}

FiguRE 1.1. POTENTIAL ROUTES OF BACTERIA AND RESISTANCE MOVEMENT AND DISSEMINATION THROUGHOUT THE ENVIRONMENT AND FOOD PRODUCTION. 16

FIGURE 1.2. ANTIMICROBIAL RESISTANCE OBSERVED IN BACTERIA ISOLATED THROUGH VARIOUS PROJECTS AT THE OTTAWA LABORATORY CARLING CANADIAN FOOD INSPECTION AGENCY (OLC-CFIA). 19

FIGURE 1.3. SELECT ANTIMICROBIAL RESISTANCE GENES OBSERVED IN BACTERIA COMMONLY FOUND IN FOOD PRODUCTS AS A FUNCTION OF ISOLATION SOURCE. .21

FIGURE 2.1. GENOME COVERAGE REQUIRED TO DETECT ARGS. ....................................57

FiguRE 2.2. EFFECTS OF SEQUENCE COVERAGE AND ASSEMBLY ON ARG DETECTION. ......60

FIGURE 3.1. IDENTIFIED RESISTANCE GENES AND GENERA....................................92

FIGURE 3.2. RESULTS OF PCR AND WHOLE GENOME SEQUENCE ANALYSES FOR B-LACTAM RELATED ANTIBIOTIC RESISTANCE GENES (ARGS) IN SEQUENCED ISOLATES FROM WASTEWATER TREATMENT PLANTS IN ONTARIO CANADA. 97

FIGURE 4.1. QUATERNARY AMMONIUM COMPOUND (QAC) RESISTANCE GENES ARE COMMON IN LISTERIA MONOCYTOGENES ISOLATES RECOVERED FROM FOOD SAMPLES.

FIGURE 4.2. PHENOTYPIC RESISTANCE TO BENZALKONIUM CHLORIDE (BC) AS A FUNCTION OF SANITIZER RESISTANCE GENE CARRIAGE. .124

FIGURE 5.1. FOODBORNE BACTERIA READILY CONJUGATE WITH A FOODBORNE PATHOGEN UNDER DIFFERENT NUTRIENT-TEMPERATURE CONDITIONS. 152 
FIGURE 5.2. CONJUGATION FREQUENCIES ARE SIGNIFICANTLY DIFFERENT BETWEEN

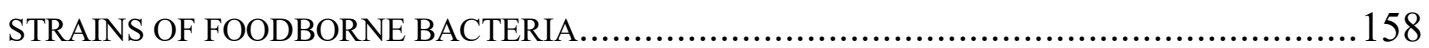

FIGURE 5.3. RATE OF PLASMID TRANSFER MAY BE HIGHER FOR MORE CLOSELY RELATED

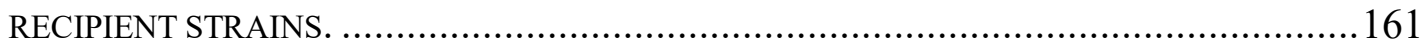

FIGURE 5.4. CONJUGATION FREQUENCY AS A FUNCTION OF DONOR-RECIPIENT RELATEDNESS. 162

FIGURE 5.5. FOODBORNE BACTERIA STABLY MAINTAIN PLASMID ENCODING BLACMY-2 FOR 100 GENERATIONS. 163

FIGURE 6.1. ACCURATE ARG DETECTION IS DEPENDENT ON ISOLATE COVERAGE IN METAGENOME

FIGURE 6.2. THE FEWER THE NUMBER OF BACTERIAL READS IN A METAGENOME, THE HIGHER THE PROPORTION THE TARGET BACTERIA MUST CONSTITUTE IN ORDER TO ACCURATELY DETECT ARGS. 


\section{List of Appendices}

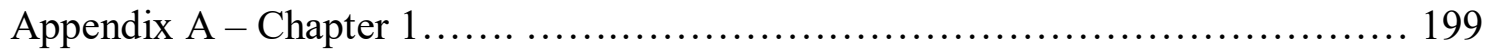

A.1 Methods for Figure 1.3 data analysis................................ 199

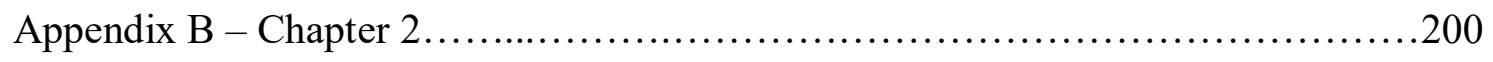

B.1 Supplementary Data File Table S2.1, Table S2.2, Table S2.3 ...........200

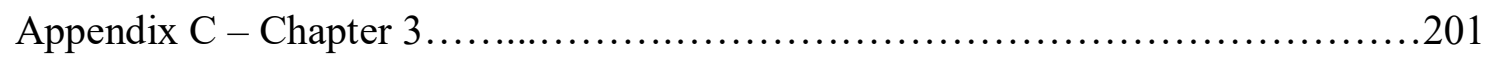

C.1 Supplementary Data Tables S3.1 and S3.2........................201

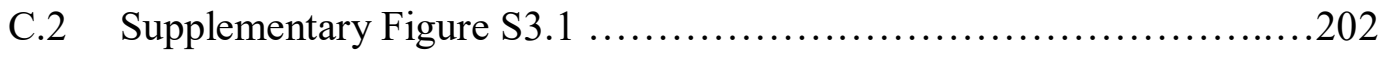

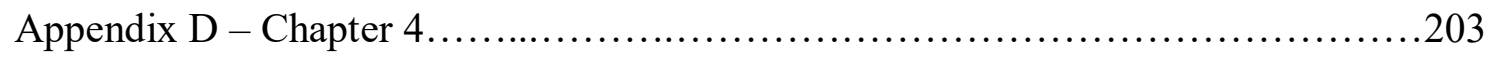

D.1 Supplementary Table S4.1 ......................................203

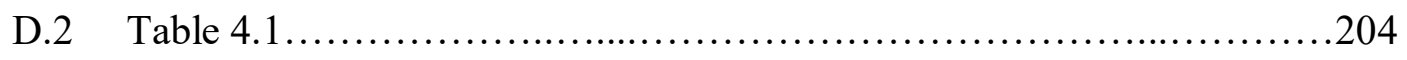

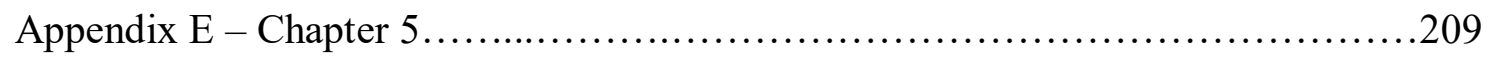

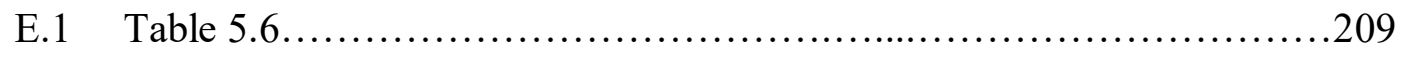




\title{
List of Abbreviations
}

\author{
$\mu \mathrm{g} \quad$ Microgram \\ $\mu 1 \quad$ Microlitre
}

AMR Antimicrobial resistance

ARG Antimicrobial resistance gene

ARO Antimicrobial resistant organism

$\beta \quad$ Beta

bp Base pair

C Celsius

CC Clonal complex

CRE Carbapenem-resistant Enterobacteriaceae

CTX cefotaxime

DNA Deoxyribonucleic acid

g Gram

HGT Horizontal gene transfer

L Litre

LOD Limit of detection

LPS Lipopolysaccharide

M Million

MGE Mobile genetic element

Mbp Million base pairs

$\mathrm{mL} \quad$ Millilitre

Nal Nalidixic acid 
NGS Next generation sequencing

OD Optical density

PCR Polymerase chain reaction

QAC Quaternary ammonium compound

Rif Rifampicin

RNA Ribonucleic acid

rRNA Ribosomal RNA

rpm Revolutions per minute

WGS Whole genome sequence 


\section{Chapter 1: General Introduction}

\subsection{Antimicrobials and antimicrobial resistance (AMR)}

Antibiotics are arguably one of the most important discoveries in the history of medicine. The discovery of sulphonamides and penicillin, and subsequent introduction of antibiotics to the field of medicine resulted in a shift in the leading causes of death from infectious diseases including pneumonia, tuberculosis, and gastroenteritis to noncommunicable diseases such as heart disease, cancer, and stroke (CDC, 2016a). In addition to treatment of infectious disease, antibiotics are also used prophylactically preand post-operatively for many surgical procedures to help decrease the risk of postoperative infections (Crader and Varacallo, 2020). In conjunction with improved hygienic practices the use of antibiotics for treatment of infectious disease led to a reduction in morbidity and mortality worldwide (Davies, 2006; CDC, 2016a) while also permitting the implementation of routine and elective surgeries, chemotherapy for cancer treatments, and transplantations (Brown and Wright, 2016). The effectiveness of antibiotics to treat and prevent life-threatening infections is critical to modern medicine (Banin et al., 2017).

Antibiotics are the largest therapeutic category used in the treatment of bacterial disease (Schmieder and Edwards, 2011). The discovery of penicillin and streptomycin in 1929 and 1943, respectively, launched "the golden era" of antibiotic discovery where many of the major drug classes currently in use were discovered (Brown and Wright, 2016). Early discovery of antimicrobials was conducted by screening libraries of chemicals and soil-dwelling bacteria for the production of metabolites that blocked or 
inhibited bacterial growth (Brown and Wright, 2016; Gould, 2016). This was followed by the synthetic era, where medicinal chemistry techniques were used to develop improved synthetic derivatives of the natural antibiotics discovered in the golden era (Brown and Wright, 2016; Hutchings et al., 2019). These synthetic derivatives had the benefit(s) of lower effective doses and broader spectra of activity. Unfortunately the distribution and use of any antibiotic has been followed by development and emergence of antimicrobial resistance (AMR), rendering some infections now untreatable (Brown and Wright, 2016; Hutchings et al., 2019). As AMR has continued to increase, the industry has been mostly unsuccessful in developing new, useful, antibiotics (Geneva: World Health Organization, 2019; Beyer and Paulin, 2020).

The frequency of AMR in clinical infections has been increasing (Andersson and Hughes, 2014; von Wintersdorff et al., 2016; Government of Canada, 2020). The World Health Organization (WHO) and the Centers for Disease Control (CDC) have each listed AMR as one of the biggest threats to global health, food security, and development (Blair et al., 2015; CDC, 2019a; WHO, 2020b). As such, many countries have developed programs for surveillance and identification of emerging AMR threats including the National Antimicrobial Resistance Monitoring System (NARMS) in the USA (CDC, 2019b), the Canadian Integrated Program for Antimicrobial Resistance (CIPARS) in Canada (Government of Canada, 2007), and the European Antimicrobial Resistance Surveillance Network (EARS-Net) in Europe (EARS-Net, 2020). These organizations monitor trends in antimicrobial use and AMR in select bacteria from human, animal and food sources to identify relationships between antimicrobials used in agriculture and medicine and health impacts associated with use (Government of Canada, 2007). 
These organizations not only monitor AMR, but also provide updates and recommendations to combat the spread of AMR both nationally and internationally.

\subsection{Antibiotic classification}

Antibiotics can be either bacteriostatic or bactericidal depending on whether they inhibit bacterial cell growth and multiplication or result in bacterial cell death. They may also be classified based on their spectrum of activity, structure, and mode of action. Broad-spectrum antibiotics are effective against a wide range of both Gram-positive and Gram-negative organisms, whereas narrow-spectrum antibiotics only have activity against a limited range of organisms. Generally, antibiotics will target cellular functions or components that are unique to prokaryotes and essential to bacterial growth including protein synthesis, replication and transcription pathways, folate synthesis, and cell wall synthesis (Table 1.1).

\subsubsection{Protein synthesis inhibition}

Protein synthesis is an essential cellular process (Lin et al., 2018). Antimicrobials that act by inhibiting protein synthesis include aminoglycosides, macrolides, phenicols, and tetracyclines, among others. These antimicrobial classes target and bind various ribosomal subunits and/or regions in such a manner as to inhibit protein translation (Lin et al., 2018; Schaenzer and Wright, 2020). 


\subsubsection{Replication inhibition}

Inhibition of replication may occur via interference with nucleic acid synthesis. Quinolones inhibit the bacterial DNA gyrase enzyme required for transcription and replication (Kapoor et al., 2017). Rifampicin inhibits the bacterial DNA-dependent RNA polymerase, preventing elongation and synthesis of bacterial proteins (Campbell et al., 2001).

\subsubsection{Folate pathway inhibitors}

Folic acid is a precursor required for nucleotide synthesis, and therefor synthesis of DNA and RNA (Kalkut, 1998; Shaikh et al., 2015). Sulphonamides and trimethoprim inhibit dihydropteroate synthetase (DHPS) and dihydrofolate reductase (DHFR), respectively, thereby blocking steps of the bacterial folate synthesis pathway (Kalkut, 1998).

\subsubsection{Cell wall synthesis inhibition}

The bacterial cell wall separates the interior of the bacterial cell from the environment, enables transport of substances in and out of the cell, and provides structural rigidity to the cell (Seltmann and Holst, 2013). Antimicrobials affecting cell wall synthesis primarily target the cytoplasmic membrane of Gram-positive bacteria, or the inner membrane of Gram-negatives (Shaikh et al., 2015). $\beta$-lactam antimicrobials inhibit cell wall synthesis by impeding enzymes required for formation of the peptidoglycan layer of the bacterial cell wall (Shaikh et al., 2015). Additional cell-wall synthesis inhibitors include glycopeptides which block the synthesis of peptidoglycan, and polymyxins which bind and destabilize LPS (Schaenzer and Wright, 2020). 
Table 1.1. Antimicrobial targets and mechanisms of action, and bacterial mechanisms of resistance to common antimicrobials

\begin{tabular}{ccccc}
\hline $\begin{array}{c}\text { Antimicrobial } \\
\begin{array}{c}\text { Mechanism of } \\
\text { Action }\end{array}\end{array}$ & $\begin{array}{c}\text { Antimicrobial } \\
\text { Class }\end{array}$ & $\begin{array}{c}\text { Example } \\
\text { Antimicrobials }\end{array}$ & $\begin{array}{c}\text { Bacterio- } \\
\text { static } / \\
\text { cidal }\end{array}$ & $\begin{array}{c}\text { Bacterial Resistance } \\
\text { Mechanism(s) }\end{array}$ \\
\hline
\end{tabular}

\begin{tabular}{|c|c|c|c|c|}
\hline \multirow{4}{*}{ Protein synthesis } & Aminoglycosides & $\begin{array}{l}\text { Streptomycin } \\
\text { Gentamicin } \\
\text { Kanamycin }\end{array}$ & Cidal & $\begin{array}{l}\text { Enzymatic modification via } \\
\text { kinases, acetyltransferases, and } \\
\text { nucleotidyltransferases; reduced } \\
\text { uptake; alteration of binding site }\end{array}$ \\
\hline & Macrolides & $\begin{array}{l}\text { Azythromycin } \\
\text { Erythromycin } \\
\text { Tylosin tartrate }\end{array}$ & Static & rRNA methylation, efflux \\
\hline & Phenicols & $\begin{array}{l}\text { Chloramphenicol } \\
\text { Florfenicol }\end{array}$ & Static & $\begin{array}{l}\text { Enzymatic inactivation via } \\
\text { acetyltransferases }\end{array}$ \\
\hline & Tetracyclines & $\begin{array}{l}\text { Tetracycline } \\
\text { Tigecycline }\end{array}$ & Static & $\begin{array}{l}\text { Efflux (tet(A)), ribosomal } \\
\text { protection (tet(M)) (Speer et al., } \\
\text { 1992) }\end{array}$ \\
\hline \multirow{3}{*}{$\begin{array}{l}\text { Cell-wall } \\
\text { synthesis }\end{array}$} & $\beta$-Lactams & $\begin{array}{l}\text { Penicillins } \\
\text { Cephalosporins } \\
\text { Carbapenems }\end{array}$ & Cidal & $\begin{array}{l}\text { Inactivating enzymes ( } \beta \text { - } \\
\text { Lactamases), PBP mutation, } \\
\text { inhibition of autolysis }\end{array}$ \\
\hline & Glycopeptides & $\begin{array}{l}\text { Vancomycin } \\
\text { Teicoplanin }\end{array}$ & Cidal & Reduced binding affinity \\
\hline & Polymyxins & $\begin{array}{l}\text { Colistin } \\
\text { Polymyxin B }\end{array}$ & Cidal & $\begin{array}{l}\text { LPS modification, increased } \\
\text { efflux, overexpression of OprH, } \\
\text { capsule formation (Olaitan et } \\
\text { al., 2014) }\end{array}$ \\
\hline DNA synthesis & Quinolones & $\begin{array}{l}\text { Nalidixic acid } \\
\text { Fluoroquinolones } \\
\text { (Ciprofloxacin) }\end{array}$ & Cidal & $\begin{array}{l}\text { Gyrase mutations, modification } \\
\text { of peptidoglycan precursors }\end{array}$ \\
\hline \multirow{2}{*}{$\begin{array}{l}\text { Inhibition of } \\
\text { folate synthesis }\end{array}$} & Sulfonamides & $\begin{array}{l}\text { Sulfasoxazole } \\
\text { Sulfadiazine } \\
\text { Sulfamethazine }\end{array}$ & Static & $\begin{array}{l}\text { Gene(s) encoding variant DHPS } \\
\text { enzymes }\end{array}$ \\
\hline & Trimethoprim & Trimethoprim & Static & $\begin{array}{l}\text { Gene(s) encoding variant DHFR } \\
\text { enzymes }\end{array}$ \\
\hline
\end{tabular}

Abbreviations: rRNA, ribosomal RNA; PBP, penicillin binding protein; LPS, lipopolysaccharide; OprH, outer membrane protein H; DHPS, dihydropteroate synthetase; DHFR, dihydrofolate reductase.

*Suffix for bacteriostatic or bacteriocidal: refers to the antimicrobial mechanism resulting in either inhibition of cell growth and multiplication (static) or bacterial cell death (cidal). 


\subsection{Antimicrobial classification according to importance in human medicine}

Although all antimicrobials are valuable and important, some are considered more important than others for clinical use. In order to assist in risk management of AMR due to non-human use, both Health Canada and the WHO have created categories based on pre-defined criteria to group antimicrobials dependent on the implications of resistance to human medicine (Government of Canada, 2009b; WHO, 2019). For example, a drug which is preferred for treatment of a serious bacterial infections (if left untreated would lead to significant morbidity), and for which there are limited or no alternatives for treatment, would be considered highly important.

Although there are overlaps between Health Canada and the WHO in the criteria and classification of antimicrobials, the category labels differ slightly. Health Canada categories consist of four levels indicated by roman numerals: I, very high importance, is a preferred option for treatment and there are limited or no alternatives available; II, high importance, is a preferred option for treatment but there are alternatives; III, medium importance, is not a preferred option, and depending on the drug there may or may not be alternative options available; IV, low importance, are not currently used in human medicine (Government of Canada, 2009b). Similarly, the WHO uses two criteria to categorize antimicrobials: criteria $1(\mathrm{C} 1)$, the antimicrobial class is one of limited available therapies to treat bacterial infection; criteria $2(\mathrm{C} 2)$, the antimicrobial class is used to treat infections in humans caused by bacteria that may be transmitted to humans from non-human sources or bacteria that may acquire ARGs from non-human sources (WHO, 2019). The WHO categorization consists of three categories: CI, critically important, meets both $\mathrm{C} 1$ and $\mathrm{C} 2$; HI, highly important, meets either $\mathrm{C} 1$ or $\mathrm{C} 2$; I, 
important, meets neither $\mathrm{C} 1$ nor $\mathrm{C} 2$. Select antimicrobial classes and their categorization

by Health Canada and WHO are compared in Table 1.2.

Table 1.2. Health Canada and WHO categorization of antimicrobial classes

\begin{tabular}{|c|c|c|c|c|c|c|}
\hline & & & & Cate & & Spectrum \\
\hline Ant & nicrobial C & & Antimicrobials & $\begin{array}{c}\text { Health } \\
\text { Canada }^{\text {a }}\end{array}$ & $\mathbf{W H O}^{b}$ & $\begin{array}{c}\text { of } \\
\text { Activity }\end{array}$ \\
\hline Aminoglyc & ides & & Gentamicin & II & $\mathrm{CI}$ & Broad \\
\hline & & & Streptomycin & II & CI & Broad \\
\hline Aminocycli & & & Spectinomycin & III & I & Broad \\
\hline Ansamycin & & & Rifampicin & I & $\mathrm{CI}$ & Broad \\
\hline$\beta$-lactams & Penicillins & & $\begin{array}{l}\text { Amoxicillin- } \\
\text { clavulanic acid }^{\mathrm{c}}\end{array}$ & I & $\mathrm{CI}^{*}$ & Broad \\
\hline & & & Ampicillin & II & $\mathrm{CI}$ & Broad \\
\hline & Cephems & $1^{\text {st }}$ gen. & Cefazolin & II & HI & Broad \\
\hline & & $2^{\text {nd }}$ gen. & Cefoxitin & II & HI & Broad \\
\hline & & $3^{\text {rd }}$ gen. & Cefotaxime & I & $\mathrm{CI}^{*}$ & Broad \\
\hline & & & Ceftriaxone & I & CI* & Broad \\
\hline & & $4^{\text {th }}$ gen. & Cefepime & I & $\mathrm{CI}^{*}$ & Broad \\
\hline & & $5^{\text {th }}$ gen. & Ceftolozane & I & CI* & Broad \\
\hline & Carbapene & & Meropenem & I & $\mathrm{CI}$ & Broad \\
\hline & & & Imipenem & I & $\mathrm{CI}$ & Broad \\
\hline & & & Ertapenem & I & $\mathrm{CI}$ & Broad \\
\hline Glycopeptic & & & Vancomycin & $\mathrm{I}$ & $\mathrm{CI}^{*}$ & Gram + \\
\hline Ketolides & & & Telithromycin & $\mathrm{I}$ & $\mathrm{CI}^{*}$ & Gram + \\
\hline Lincosamid & & & Clindamycin & II & $\mathrm{HI}$ & Gram + \\
\hline Phenicols & & & Chloramphenicol & III & HI & Broad \\
\hline Polymyxins & & & $\begin{array}{l}\text { Colistin } \\
\text { (polymyxin E) }\end{array}$ & I & $\mathrm{CI}^{*}$ & Broad \\
\hline Quinolones & & & Nalidixic acid & II & CI & Gram - \\
\hline & Fluoroquin & lones & Ciprofloxacin & I & CI* & Broad \\
\hline Macrolides & & & Azithromycin & II & $\mathrm{CI}^{*}$ & Broad \\
\hline & & & Erythromycin & II & $\mathrm{CI}^{*}$ & Broad \\
\hline Tetracyclin & & & Chlortetracycline & III & HI & Broad \\
\hline & Glycycline & & Tigecycline & I & CI & Broad \\
\hline
\end{tabular}


Abbreviations: WHO, World Health Organization; Cephems, cephalosporins; gen., generation; Gram +, Gram-positive; Gram -, Gram-negative.

${ }^{a}$ Health Canada categorization based on importance in human medicine: I, extremely high importance; II, high importance; III, medium importance.

${ }^{b}$ WHO categorization based on importance in human medicine: CI, critically important; HI, highly important; I, important.

${ }^{\mathrm{c}}$ Amoxicillin Is a beta-lactam, potassium clavulanate is a beta-lactamase inhibitor.

*WHO has categorized these as being the highest priority critically important antimicrobials.

\subsection{Antimicrobial resistance mechanisms}

Bacteria have evolved a variety of strategies to avoid the effects of antibiotics. These AMR mechanisms can be either intrinsic or acquired. Intrinsic AMR refers to natural mechanisms inherent to a microorganism, for example due to absence of an antimicrobial target. Acquired AMR occurs when a microorganism evolves or obtains resistance mechanisms that include resistance genes on plasmids, mutations in regulatory elements and housekeeping genes, and insertion of active promoters upstream of a resistance gene (Anjum et al., 2017). Generally, known resistance mechanisms include minimizing intracellular concentrations of the antibiotic, modification of antimicrobial target(s), and antimicrobial inactivation (Table 1.1).

\subsubsection{Diminishing access to antimicrobial target via influx/efflux modification}

Increased efflux of the antimicrobial from the cell or decreased influx of the antimicrobial into the cell may be used as bacterial resistance mechanisms. Bacterial efflux pumps are transport proteins that move toxic compounds from the bacterial cell out into the environment. Overexpression of efflux pumps can confer resistance by faster more effective export of antimicrobials out of the cell (Webber and Piddock, 2003; Blair et al., 2015). Efflux pumps may be specific to antimicrobials, however most of them are 
multidrug transporters able to transport many different antimicrobials and conferring MDR (Kapoor et al., 2017). As porins are required by some hydrophilic antimicrobials, such as $\beta$-lactams and quinolones, to cross the outer bacterial membrane, decreasing the number of porins also leads to decreased entry of the antimicrobial into the cell (Kapoor et al., 2017).

Lázár et al. (2013) reported that evolutionary adaptation resulting in aminoglycoside resistance was often accompanied by hypersensitivity to other antimicrobials. Proton motive force (PMF) is important for cellular uptake of aminoglycosides which contrasts with other antimicrobials which have PMF-dependent efflux. These resistance conferring mutations may currently be more difficult to detect as they are often conferred by single nucleotide variants (SNVs) in the bacterial genome.

\subsubsection{Modification of antimicrobial target(s)}

Bacterial modification of the antimicrobial target is becoming increasingly prominent in clinical isolates (Schaenzer and Wright, 2020). This mechanism has been observed as a bacterial response to glycopeptides, polymyxin, rifamycins, and ribosome targeting antimicrobials including aminoglycosides and phenicols (Table 1.1) (Reviewed by Schaenzer and Wright, 2020). Resistance to polymyxins and glycopeptides is achieved by altering the cellular envelope to prevent binding and entry of the antimicrobial. Glycopeptides modify the terminal D-alanine residue of the pentapeptide stem in peptidoglycan precursors to alter hydrogen bonding, thereby preventing the glycopeptide from blocking peptidoglycan synthesis (Schaenzer and Wright, 2020). Similarly, polymyxin resistance may be conferred via LPS modification via addition of functional 
groups that mask Lipid A with positive charges thereby repelling polymyxins (which are cationic) (Schaenzer and Wright, 2020). Bacteria may also produce methyltransferases that modify the ribosome to prevent binding of antimicrobials such as aminoglycosides and macrolides. Methylation results in weakened interactions between the antimicrobial and ribosome by modification of the antimicrobial binding site. In contrast loss of methylation is a resistance mechanism employed by Mycobacterium tuberculosis and against the aminoglycoside kasugamycin (Johansen et al., 2006; Schaenzer and Wright, 2020). As with methylation, loss of methylation results in ribosome modification at the antimicrobial binding site.

Overproduction of the antimicrobial target is a response characteristic of the folate pathway inhibitor sulfonamides and trimethoprim. Resistant bacteria may have compensatory mutations in DHPS or have acquired drug-resistant forms of DHPS (Sköld, 2000).

\subsubsection{Modification or inactivation of the antimicrobial}

Bacteria may produce proteins or enzymes that modify or inactivate antimicrobials. This mechanism has been documented as a response to aminoglycosides, phenicols, $\beta$-lactams, glycopeptides, and quinolones (Table 1.1). One of the most prevalent and well-studied bacterial resistance mechanisms is inactivation of $\beta$-lactam antimicrobials via production of $\beta$-lactamases. $\beta$-lactamases are hydrolases including cephalosporinases, penicillinases, and carbapenemases, that inactivate $\beta$-lactam antibiotics via hydrolysis of the $\beta$-lactam ring structure. Similarly, resistance to aminoglycosides may occur through generation of aminoglycoside modifying enzymes 
such as acetyltransferases (AACs), adenyltransferases or nucleotidyltransferases (ANTs), and phosphotransferases (APHs). These aminoglycoside modifying enzymes catalyze modification at $-\mathrm{OH}$ or $-\mathrm{NH} 2$ groups of the aminocyclitol nucleus or sugar moieties of aminoglycoside antimicrobials (Ramirez and Tolmasky, 2010).

\subsection{Biocides and biocide resistance}

Biocides are sanitizers that contain chemical agents used for a variety of purposes including to make water and foods safe, treat wounds, and disinfect. They are used in many industries including food production, water treatment, farming, and health care. Like antibiotics, biocides have different modes of action against different organisms (Gupta et al., 2018). Recent increases in reduced bacterial susceptibility to biocides has been observed (Dutta et al., 2013; Wales and Davies, 2015; Ortiz et al., 2016). Genes encoding resistance to biocides, including quaternary ammonium compounds (QACs), have been observed in Gram-negative and Gram-positive bacteria (Kücken et al., 2000; Xu et al., 2016; Romero et al., 2017; Gupta et al., 2018; Vijayakumar and Sandle, 2019). As biocide resistance may develop through increased efflux, or acquisition of mobile genetic elements (MGEs) encoding resistance genes, there is concern that development of bacterial biocide resistance may also result in increased bacterial AMR. Co-selection of biocide, metal, and antimicrobial resistance has been observed among pathogens and other bacteria (Wales and Davies, 2015). 


\subsection{Drivers of resistance}

Acquisition of ARGs mainly occurs through horizontal gene transfer (HGT). The main mechanisms of HGT include conjugation, transformation, and transduction (von Wintersdorff et al., 2016). ARG transfer between bacterial genera and species often occurs via conjugation, as mobile genetic elements (plasmids, phages, and transposons) often encode genes that confer a selective advantage such as resistance genes. The presence of low or subinhibitory levels of antibiotic in the environment may promote selection and gene transfer, and recent studies have suggested that infection and conditions mimicking the mammalian gut may enhance spread of plasmid-encoded resistance (Stecher et al., 2012; Van Meervenne et al., 2012; Aviv et al., 2016).

Overuse of antimicrobials in both medicine and agriculture are proposed drivers of the increase in antimicrobial resistance as subinhibitory concentrations of antimicrobials may create a selective pressure for resistant bacteria (Holmes et al., 2016). However, studies have contested agriculture's contribution to AMR (Phillips et al., 2004; Kirchhelle, 2018). Thomas et al. (2017) did not observe any correlation between administration of antibiotic feed and presence of ARGs in the cattle gut microbiome. Duffy et al. (2014) reported that streptomycin use in apple orchards did not increase the abundance of mobile streptomycin $(\operatorname{str} A, \operatorname{str} B)$ and tetracycline (tet $B$, tet $M$, tet $W)$ ARGs. In addition, a study by Udikovic-Kolic et al. (2014) reported that blooms of AMR bacteria in soil following manure fertilization was independent of antimicrobial exposure. In contrast, Brown et al. (2019) demonstrated that medicated animal feeds could select for AMR bacteria, and even resulted in co-selection of a multi-drug resistant (MDR) Klebsiella pneumoniae which encoded the $b l a_{\mathrm{KPC}-3}$ allele for carbapenem resistance. 
Pornsukarom and Thakur (2017) also found different AMR carrying plasmids persisted and transferred between bacteria following swine manure application. Conflicting reports regarding the development and transfer of bacterial resistance, regardless of presence/absence of antimicrobials, highlights the importance of studying the spread of resistance within the food production continuum to help provide clues as to how it can be controlled.

\subsection{The Food Production continuum}

Food production is conducted along a continuum from processes involving manufacture and production to consumption (farm-to-fork or farm-to-table). The amount of processing depends on the product where those that require little processing such as vegetables are labelled minimally processed and others may be subject to varying levels of processing including addition of preservatives, sweeteners, spices, and coloring, to "ready-to-eat" foods which need minimal or no preparation prior to consumption (such as breakfast cereal, yogurt, and deli-meats). Along the continuum there are many points for possible microbial contamination of both agri-food products and processing equipment, as well as the potential for cross-contamination between products (e.g. improper food hygiene where the same knife is used to cut raw-chicken and vegetables).

Food crops and animals harbor bacteria that are pathogenic to humans (Riley, 2020), and the spread of bacteria from these sources to food products is extensively documented and monitored for pathogens including Salmonella spp., Escherichia coli, and Listeria monocytogenes (Wegener, 2003; Cantas et al., 2013; Bintsis, 2017; Riley, 2020). These pathogens as well as commensal bacteria of food microbiota(s) can inhabit 
and spread between multiple environments including agricultural, aquatic, and clinical settings, where they could potentially acquire and transmit AMR (Figure 1.1). For example, soil is used to grow food crops to feed both humans and animals. Soils may be amended with fertilizers to supply nutrients required for plant growth. These fertilizers may be obtained from a variety of sources including composted wastes such as manure or biosolids, which are organic materials derived from wastewater treatment plant sewage sludge. Many of the fertilizers used in agriculture are derived from human and animal waste products and are potential sources of bacterial contamination. Bacteria in soil and plants may disseminate to crops growing in fertilizer-amended soils and to water sources via agricultural runoff (Figure 1.1). Potentially contaminated crops may be consumed directly by humans, be incorporated into other food products, or be used in feed for food producing animals (Figure 1.1). Subsequently, bacteria can then travel further along the food production continuum to consumers as well as to the gut of animals and possibly to meat products and production lines.

Antimicrobials are used to treat disease in agriculture and food animal production, to increase feed efficacy, as growth promoters, and prophylactically to prevent disease circulation (Landers et al., 2012; Kirchhelle, 2018; Kimera et al., 2020). Antimicrobial use in agriculture is necessary for plant, animal, and human health, as livestock production often requires high population densities. Practical administration of antimicrobials to large animal populations is usually achieved through either water or animal feed (Wegener, 2003).

In addition to antimicrobial use, biocides, disinfectants or sanitizers are often utilized during food production. Generally, biocides are defined as substances formulated 
to be harmful to living organisms (Wales and Davies, 2015). For the purposes of this thesis biocides will refer to disinfectants and sanitizer products with more varied applications, which are not selective enough to be used within body tissues, but will not include antimicrobials (many of which are technically harmful to living microorganisms). Biocides are used to clean and disinfect equipment and surfaces during health care, farming, and food production; as decontaminants on carcass surfaces and medical equipment; and as preservatives in cosmetics, pharmaceuticals, and foods in order to control pathogenic and spoilage microorganisms (Holah et al., 2002; Wales and Davies, 2015).

With the development of compounds with limited toxicity for humans and animal tissues, such as quaternary ammonium compounds (QACs), biocides are increasingly used for disinfection (Wales and Davies, 2015). As with AMR, spread of resistance to sanitizers used in food production has also been observed (Oniciuc et al., 2019). Use of biocides and preservatives may increase ARG transfer among bacteria as well as coselect for MDR strains (Futoma-Kołoch et al., 2015; Wales and Davies, 2015; Webber et al., 2015; Monte et al., 2019; Oniciuc et al., 2019). Studies have reported an association between biocide use in poultry and egg production and isolation of biocide tolerant and AMR Salmonella spp. (Fernández Márquez et al., 2016; Márquez et al., 2018; Rhouma et al., 2020). Nonetheless, as with AMR, some research suggests repeated disinfectant use in food processing and agricultural environments does not select for biocide nor antimicrobial resistance (Gantzhorn et al., 2014; Maertens et al., 2020). 


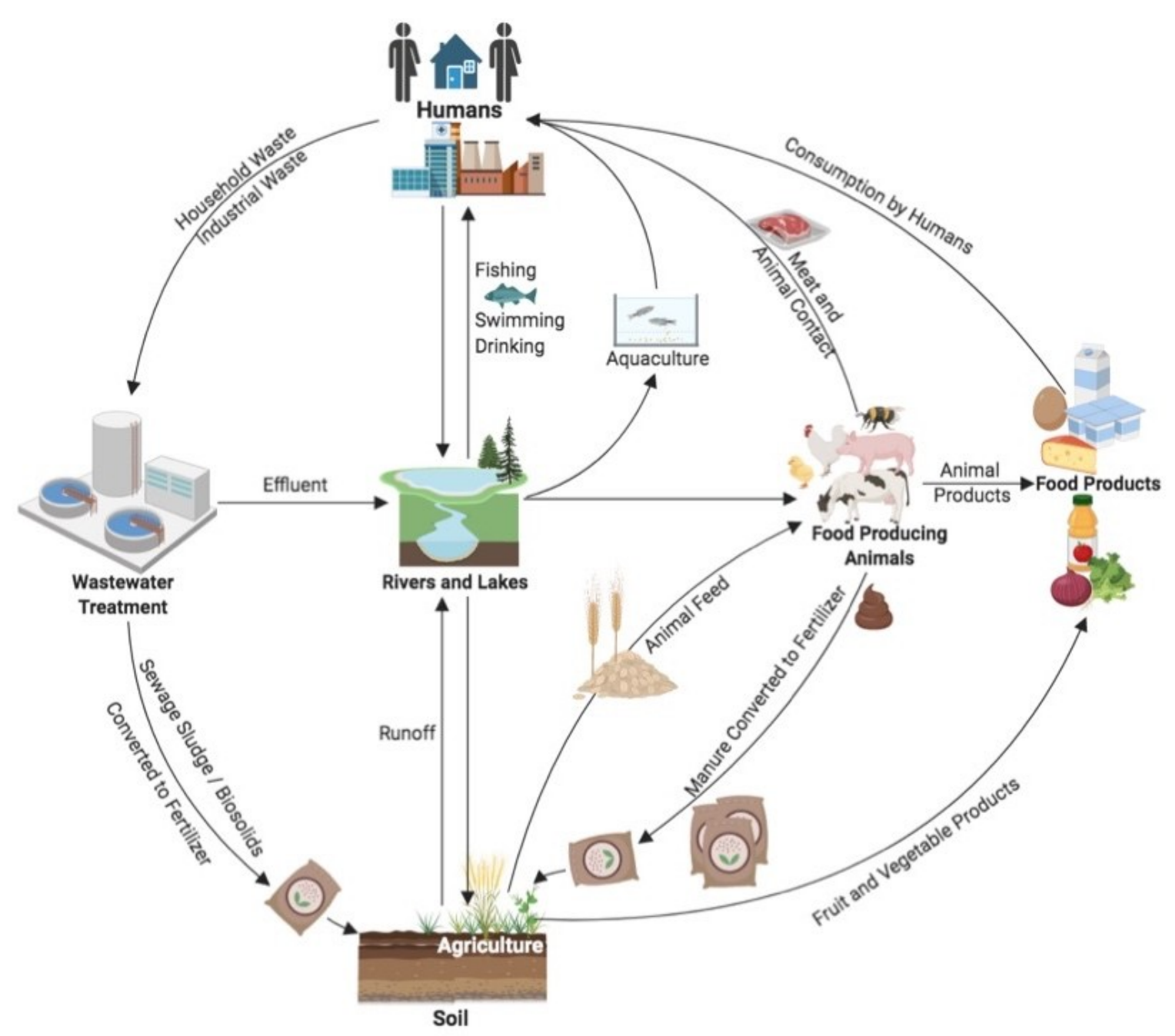

Figure 1.1. Potential routes of bacteria and resistance movement and dissemination

throughout the environment and food production. Arrows indicate points of

dissemination among different environments. Humans represents all human-related activities including clinical, industrial, and household. Intricacies of food production processes including processing, pasteurization, slaughter, sanitization, packaging, preparation, etc., are not displayed but are inferred by arrows from agriculture to food products, from aquaculture to humans, and from animals to both food products and humans. (Figure is adapted from Schmieder and Edwards, 2011. Created using BioRender.com) 


\subsection{Filling the gaps in agri-food testing and resistance surveillance}

The Canadian Food Inspection Agency (CFIA) is the regulatory agency responsible for safeguarding and monitoring foods, animals, and plants in Canada. Current regulations monitored and enforced by the CFIA only require testing for the presence of select clinically relevant pathogens within products (e.g. Salmonella spp., verotoxigenic E. coli, and Listeria monocytogenes). As it is likely that non-pathogens within animal, plant, and food product microbiomes also carry resistance determinants, policies and methods that only address a small number of pathogenic bacteria may not be sufficient to accurately detect the total resistance burden.

The WHO and CDC list carbapenem and ESBL resistant Enterobacteriaceae and drug-resistant Salmonella as AMR priority pathogens (CDC, 2019a; WHO, 2020). Food products, facilities, and food producing animals contain a variety of Enterobacteriaceae and Listeria monocytogenes, among other bacteria, and antimicrobial use in agriculture has been alleged as a driver for increasing AMR. It is possible resistance surveillance in the food production continuum could leverage current isolation activities already conducted by the CFIA, as food inspection activities already target Salmonella and E. coli (other tested genera are not discussed here). Yet, a very low percentage of the Salmonella and $E$. coli recovered during routine regulatory food testing encode AMR genes (Figure 1.2). In contrast, other genera such as Citrobacter, Enterobacter, Hafnia, Klebsiella, and Proteus which are commonly isolated from agri-food samples more often exhibit AMR (Figure 1.2). However, not all ARGs are of equal importance. For example, chromosomal $\beta$-lactamase genes are encoded by Citrobacter $\left(\right.$ bla $\left._{\mathrm{CMY}}\right)$, Enterobacter $\left(\right.$ bla $\left._{\mathrm{ACT}}\right)$, and Hafnia $\left(\right.$ bla $\left.a_{\mathrm{ACC}}\right)$ which may confer resistance to $1^{\text {st }}$ - and $2^{\text {nd }}$ - generation cephalosporins 
( $\beta$-lactams), but not to $3^{\text {rd }}$ - and $4^{\text {th }}$ - generation cephalosporins which are on the list of critical importance (Table 1.2, Figure 1.2).

Analysis of AMR in WGS data from the National Center for Biotechnology Information (NCBI) Pathogen Detection database (available at https://www.ncbi.nlm.nih.gov/pathogens/), which contains far more data than the OLCCFIA collection, also found a lower percentage of Salmonella and E. coli from food products encoded some critically important ARGs in comparison to other foodborne genera (Figure 1.3). Interestingly, for some antibiotic classes many foodborne isolates in NCBI Pathogens exhibited elevated levels of resistance (Figure 1.3, sul and tet). In addition, higher rates of streptomycin resistance were observed in Salmonella isolated from meat sources (Figure 1.3, $a a d A$, $a p h(3$ ') $)-I b$, and $a p h(6)-I d)$. Yet, high rates of resistance observed in clinical isolates of some genera do not correlate with rates of resistance from other sources (Figure 1.3). As we do not see an abundance of critical AMR in routinely monitored foodborne pathogens, it is important to also investigate other foodborne bacteria to determine possible AMR burdens and dissemination from the food production continuum. 
Figure 1.2. Antimicrobial resistance observed in bacteria isolated through various projects at the Ottawa Laboratory Carling Canadian Food Inspection Agency (OLC-CFIA). (Page 20) For each genus listed on the y-axis, percentage of isolates from each project with resistance to antimicrobial class (panel heading) is displayed (x-axis). Sizing of points corresponds to the total number of isolates of that genus sampled for corresponding project (Number of Isolates). Results for Regulatory, F-1701, and F-1407 projects are from analysis of whole genome sequence data, whereas results for F-1600 are from phenotyping of isolates. Projects are differentiated by color: blue) CFIA Regulatory, agri-food samples are only tested for verotoxigenic E. coli and Salmonella; orange) Project F-1701, bacteria isolated from various foods using non-selective culture methods then analysed for AMR following whole genome sequencing; green) Project F1407, bacteria isolated from foods following mTSB enrichment then analysed for AMR following whole genome sequencing; red) Project F-1600, bacteria isolated from agrifood products and phenotyped (for AMR) following mTSB enrichment. 


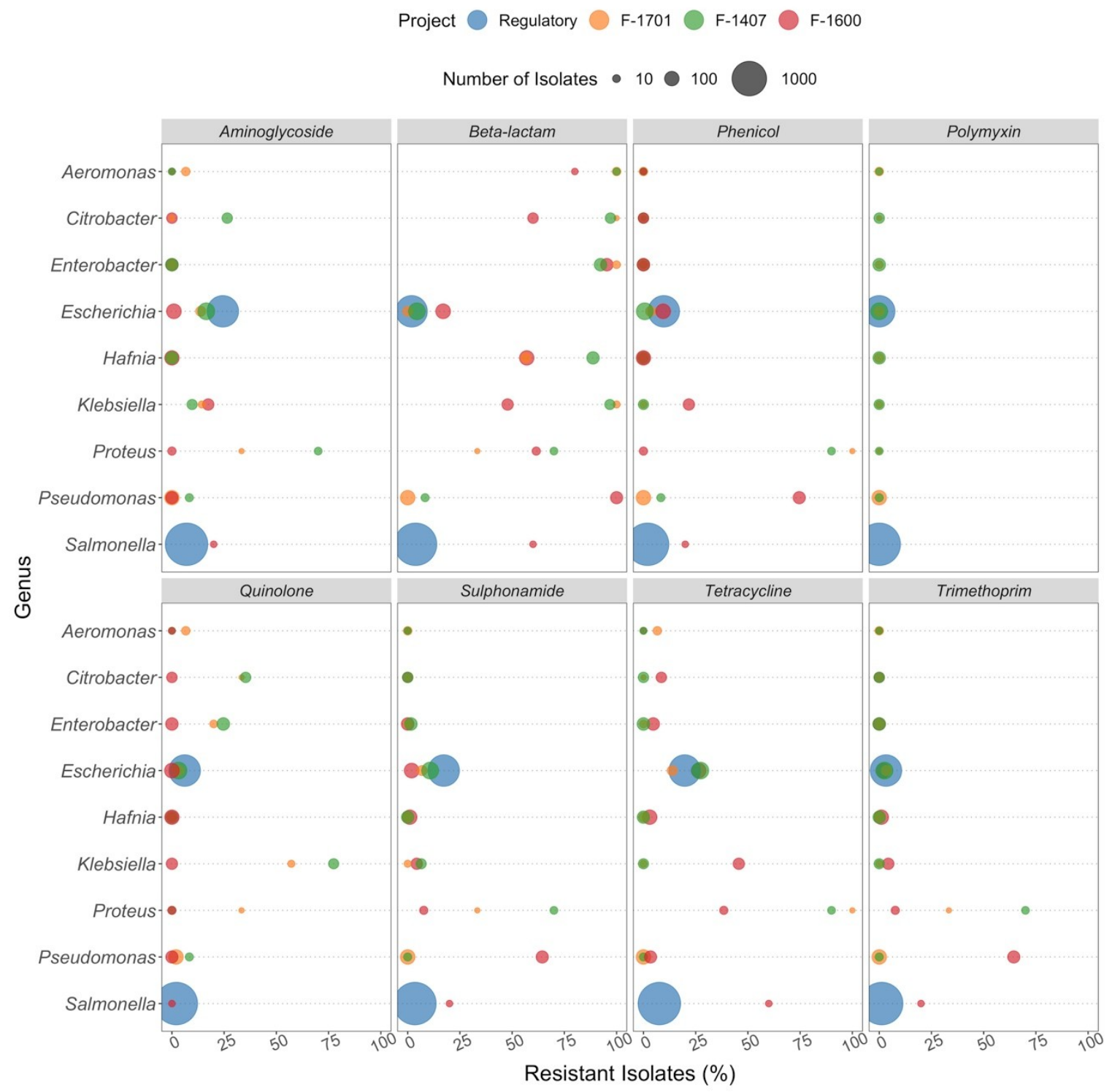


Figure 1.3. Select antimicrobial resistance genes observed in bacteria commonly found in food products as a function of isolation source. (Page 22) For each genus listed on the $y$-axis, percentage of isolates from each source (see legend) with each respective ARG (panel headings) is displayed (x-axis). Sizing of points corresponds to the total number of isolates of that genus for which isolation source could be identified (Number of Isolates). Metadata files for whole-genome sequences were downloaded from the NCBI Pathogen Detection database on August 26, 2020 (https://www.ncbi.nlm.nih.gov/pathogens/). Datasets were analysed and source categories were manually assigned based on provided sequence submission information. Data was excluded from analysis where source information was missing or incomplete. Methods for data analysis are in Appendix A.1. 


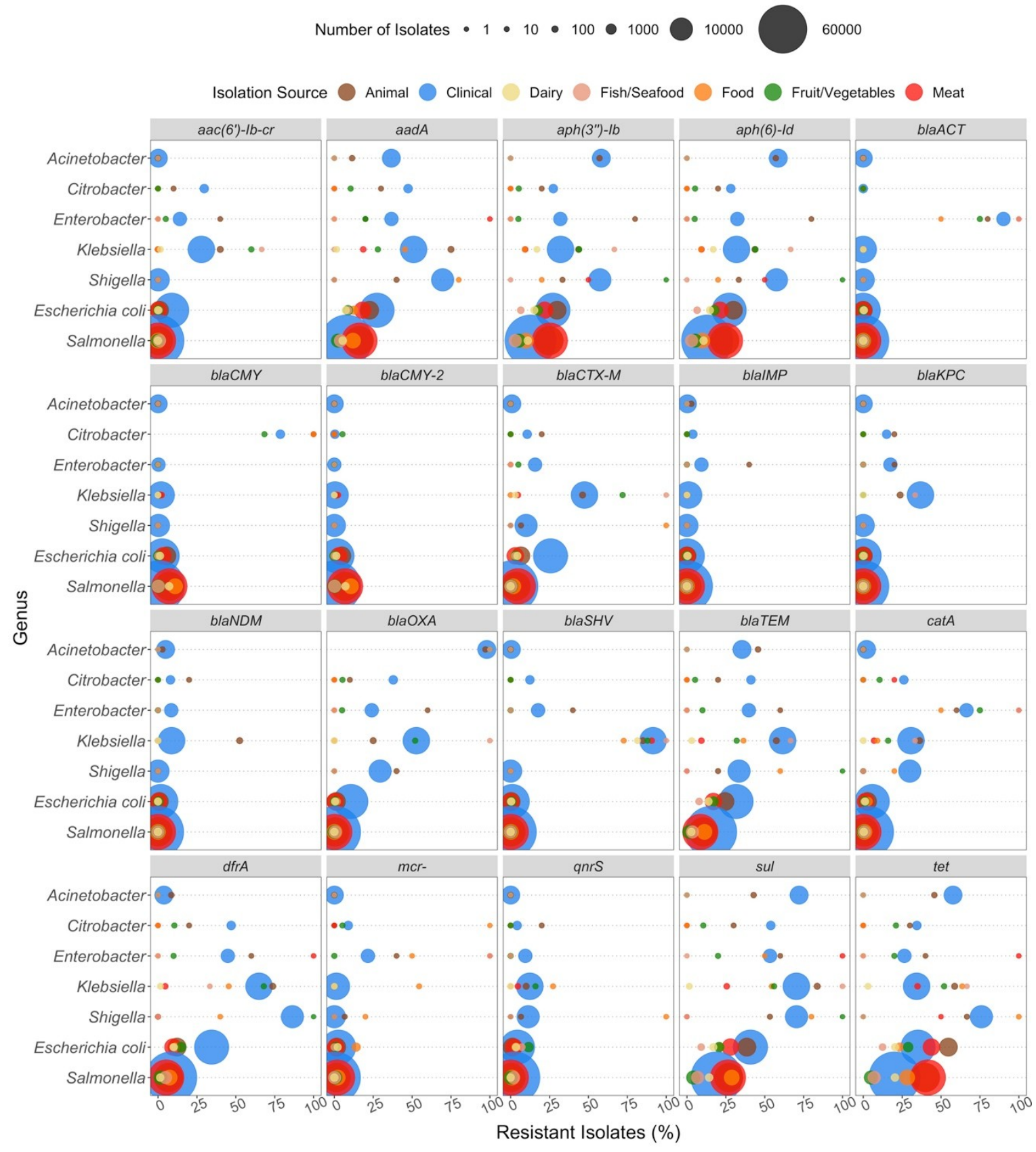




\subsection{Detection of resistance}

ARG and biocide-resistance detection is achievable using a variety of different phenotypic and molecular methods. Phenotyping methods for AMR include a number of published standardized protocols utilizing growth inhibition assays (Qi et al., 2006;

Wiegand et al., 2008). These methods are used to determine drug susceptibility, or the minimum inhibitory concentration (MIC) of antimicrobial or biocide required to inhibit bacterial growth and detect enzymatic activity in tested isolates (e.g. carbapenemase production). Interpretive standards exist for AMR to classify organisms as clinically susceptible, intermediate, or resistant, and are published by the Clinical Laboratory Standards Institute (CLSI) in North America, and the European Committee on Antimicrobial Susceptibility Testing (EUCAST) in Europe (Wiegand et al., 2008; Clinical and Laboratory Standards Institute (CLSI, 2020; EUCAST, 2020). Unfortunately, culture-based approaches exclude unculturable isolates and are laborious.

Molecular techniques often investigate bacterial resistance by targeting known resistance genes. Common techniques include PCR, quantitative or real-time PCR (qPCR), hybridization techniques, high resolution melting curve analysis, and matrixassisted laser desorption ionization-time of flight mass spectrometry (MALDI-TOF MS) (Fluit et al., 2001; Rosengren et al., 2009; Licker et al., 2015; Anjum et al., 2017; Sirous et al., 2018). However, these approaches are also limited to analysis of well-studied organisms or resistance-causing genes and are not always useful for screening a large number of targets. Additional limitations arise due to the large number of variable genealleles of known ARGs, making development of all-encompassing assays for a single 
gene almost impossible. In addition, discovery of novel ARGs may result in the need to design additional assays and re-analyse samples.

Whole-genome sequencing (WGS) is another method employed to detect genetic determinants including genes conferring virulence and AMR. Studies conducted by NARMS evaluated the ability of WGS to predict AMR phenotypes in Salmonella and $E$. coli using an in-house ARG database found AMR genotypes and phenotypes correlated 99\% (Tyson et al., 2015, 2016; McDermott et al., 2016). The high concordance between phenotype and genotype has led to production of machine learning algorithms that correlate phenotypes with genomic variations (Macesic et al., 2017; Drouin et al., 2019; Liu et al., 2020; Van Camp et al., 2020).

\subsubsection{Next generation sequencing approaches}

As mentioned, a major disadvantage of culture-based techniques is that there is still a substantial number of microorganisms that have not yet been cultured (Jernberg et al., 2010; Hug, 2018; Steen et al., 2019). Though culture-based techniques are useful, high-throughput sequencing-based approaches have the potential to bypass some of the limitations of culturing by allowing simultaneous community fingerprinting and resistance gene detection of the microorganisms in complex sample matrices (Nayfach and Pollard, 2016).

High-throughput 16S (rRNA) and cpn60 (type I chaperonin) sequencing may be used to infer taxonomy of a sample's microbial population (Links et al., 2012; Kryachko et al., 2017; Johnson et al., 2019). Whereas $16 \mathrm{~S}$ techniques allow community profiling by targeting and amplifying a specific gene within a sample, shotgun metagenomics (the 
untargeted sequencing of everything in a sample) is advantageous as it "ideally" enables genomic analyses and identification of all microorganisms in a sample including resistance genes. However, when a sample of metagenomic DNA is sequenced that sample is assumed to be a random fraction of the total sample's microbial community. This is a limitation of shotgun metagenomics, as differences in species composition and abundance could be present (Sogin et al., 2006; Hugerth and Andersson, 2017) and therefore ARG abundance within a metagenomic sequence may be limited. Fitzpatrick and Walsh (Fitzpatrick and Walsh, 2016) investigated the distribution of ARGs across soil, water, animal, plant, and human microbiomes using metagenomic sequence data available in the Metagenomics Rapid Annotation using Subsystem Technology (MGRAST) database (Keegan et al., 2016). Though they found that human and mammalian gut microbiomes contained the widest diversity of clinically relevant ARGs, they caution that other ecological niches may harbour ARGs at levels below the limits of current techniques (Fitzpatrick and Walsh, 2016). Yet, we still understand little about the distribution of ARGs throughout different environments (Singer et al., 2016; Stanton et al., 2020).

Some work has been conducted to investigate the plasmidome, or entire plasmid content, of complex sample matrices (Zhang et al., 2011; Brown Kav et al., 2012; Dib et al., 2015; Kothari et al., 2017; Perez et al., 2020). When environmental changes induce stress, bacteria may benefit more quickly from horizontal gene transfer of resistance than mutation and selection (Kothari et al., 2017). Perez et al. (2020) reported that genetic information from sequencing of the plasmidome was novel in comparison to shotgun metagenomics of the same environment (only $30 \%$ of the plasmidome reads aligned with 
metagenomic reads). As plasmids provide a mechanism to distribute genes beneficial for survival the plasmidome is likely to contain an abundance of genes encoding virulence factors; antimicrobial-, biocide-, and metal-resistance; and functions involved in hostbacterial interactions (Dib et al., 2015). Therefore, the plasmidome may (arguably) contain resistance genes of highest risk and clinical relevance in food production samples.

Advancements in next generation sequencing (NGS) technologies has enabled many laboratories to conduct genomic analyses in-house. As with any laboratory method, NGS methods also have principles and guidelines for quality control (QC) to ensure accuracy of results reported based on NGS (Gargis et al., 2012). Three metrics used to evaluate sequence quality include depth of coverage, base quality, and mapping quality. Depth of coverage refers to how many unique sequence reads align to, or "cover", a given nucleotide when making a base call (Sims et al., 2014). Base quality is whether the correct bases have been called in a sequence read, and mapping quality is whether the reads have been mapped to the correct position in the genome (Gargis et al., 2012; Münz et al., 2018). Although these metrics are often used to evaluate WGS data, metagenomes do not have a "fixed end point" (Kunin et al., 2008), as in not all organisms in the sequence are known and assembly from the fragments of each of the organism genomes that may be present in the metagenome is extremely difficult. Therefore, metagenomes do not always necessarily follow guidelines for depth of coverage and mapping quality, as sequencing coverage and mapping quality could vary widely within and between metagenomic sequences. 


\subsection{Current investigations and major thesis objectives}

As samples found throughout the food continuum are often compositionally complex, methods that will enable evaluation of resistance burden in the food chain are required. The major objectives of this thesis were to fill in current knowledge gaps through evaluating methods, testing, and evaluating resistance relevant to food production. This will enable contributions to current surveillance by enabling evaluation of potential health risks caused by bacterial resistance in the food production continuum. The main focus of this work was on ARGs that may be acquired or transferred throughout the food production continuum. The hypotheses and specific goals of this thesis are summarized below.

\section{Objective 1: Testing the concordance between genotype and phenotype in predicting}

\section{$A M R$}

Hypotheses for this objective include:

1) AMR phenotype can be accurately predicted using isolate WGS data, granted sufficient sequence coverage is achieved.

2) AMR genes are better detected in raw-reads than assembled sequence data.

Chapter 2 investigates the concordance of genotypic predictions of phenotypic AMR in the foodborne pathogen Salmonella enterica, evaluating the performance a variety of publicly available bioinformatic tools and ARG databases. Although a number of studies exist investigating the utility of WGS to predict AMR, not all of them fully explored potential causes of observed discrepancies in genotype-phenotype predictions 
which may be due to SNVs. This study also investigated the sequencing requirements (depth of coverage, assembly versus raw-read sequence data) for accurate ARG detection using the Salmonella WGS data. The findings from this chapter have implications for strategies to detect AMR using sequencing technologies, as certain coverage thresholds should be met for accurate predictions.

Chapter 3 is similar to Chapter 2 in that it compares phenotypic carbapenem resistance with PCR and WGS techniques for gene detection. This study also compared two publicly available ARG detection tools, ResFinder and CARD-RGI. Both tools were unable to detect carbapenem-resistance genes in a number of isolates when using the default target gene-identity threshold of $\geq 90 \%$. Lowering the target-identity to $\geq 80 \%$ allowed detection of closely related alleles in the ARG-database for some of the tested isolates. This study highlights the utility of WGS over PCR for ARG detection where resistance may be the result of a multitude of genes (e.g. $\beta$-lactam resistance genes) that would require many multiplex PCRs. It also confirms that genotypic predictions of AMR phenotype are accurate for well-studied organisms, such as the Enterobacteriaceae.

\section{Objective 2: Investigate the mechanisms of bacterial resistance in food production}

Hypotheses for objective 2 include:

1) Use of sanitizers in food production select for resistance which leads to persistence of foodborne pathogens.

2) Foodborne bacteria, including non-pathogens, facilitate transfer of ARGs 
Chapter 4 is an investigation of the correlation of bacterial persistence of the foodborne pathogen L. monocytogenes in food production due to biocide resistance. This study analysed sequence data of 1279 L. monocytogenes isolated from a variety of food products and manufacturing environments as part of routine testing at the CFIA. This chapter highlights that extensive use of antimicrobial compounds such as biocides during food processing may select for persistence of bacteria encoding resistance genes. It confirms that $L$. monocytogenes frequently isolated from food production sources where sanitizers are frequently utilized for disinfection are statistically more likely to encode biocide resistance genes. It suggests the possibility that overuse and subinhibitory QAC concentrations create a selective pressure for this resistance in some food commodities.

Chapter 5 investigates the role of foodborne bacteria in facilitating transfer of AMR via conjugative transfer of a plasmid encoding resistance to $3^{\text {rd }}$ generation cephalosporins under different conditions. Although studies investigating conjugation are plentiful, most utilize clinical or laboratory bacterial strains as donors and recipients. This chapter has confirmed that different genera of foodborne Enterobacteriaceae can receive and stably maintain clinically relevant $\mathrm{ARGs}$ in both minimal and rich media at $25^{\circ} \mathrm{C}$ and $37^{\circ} \mathrm{C}$. Observed conjugation frequencies of some strains were quite high and comparable to that of the control laboratory E. coli. Also of note was the effect of the antimicrobial concentration, where if the concentration used to select transconjugants is too high (e.g., $4 \mu \mathrm{g} / \mathrm{ml}$ cefotaxime, which is the MIC) it may misrepresent the actual rate of plasmid transfer occurring between foodborne isolates. This study confirms that foodborne bacteria conjugate with foodborne pathogens, sometimes at elevated rates, and reinforces that foodborne microbiota should also be studied and monitored for resistance genes. 


\section{Objective 3: Determine suitable methods to monitor resistance in agri-food production}

The hypothesis tested for this objective is:

1) ARGs in agri-food samples are present below the limit of detection of metagenomics.

Chapter 6 is a pilot study examining the limit of detection for ARGs in short-read shotgun metagenomic sequence data. This chapter leverages the knowledge from chapter 2 to investigate the limit of detection of antimicrobial resistance in agri-food metagenomic samples. Although metagenomic sequencing is increasingly being used to investigate AMR in different environments it is important to understand and acknowledge the limitations of this technology. Whereas many clinical specimens, such as blood and urine, are relatively "clean", samples from agri-food production are often complex in nature, potentially containing many different eukaryotic and prokaryotic cells. This chapter shows that metagenomic sequence data requires an organism to be present at approximately 10X genome coverage if ARGs are to be accurately detected.

Understanding the limit of detection of ARG detection methods is crucial if these methods are to be used for surveillance and risk assessment. This study highlights that the lower the number of sample sequence reads, the larger percentage of the sample the target organism(s) must comprise if ARGs they harbour are to be detected.

Chapters 7 and 8 are the concluding chapters that summarizes the principal findings of this thesis and ties together the importance of the results of previous chapters. 


\section{Chapter 2: Systematic Evaluation of Whole Genome Sequence-Based \\ Predictions of Salmonella Serotype and Antimicrobial Resistance}

This chapter is an adaptation of the following publication:

Cooper, A.L., Low, A.J., Koziol, A.G., Thomas, M.C., Leclair, D., Tamber, S., Wong, A., Blais, B.W. \& Carrillo, C.D. (2020). Systematic Evaluation of Whole Genome Sequence-Based Predictions of Salmonella Serotype and Antimicrobial Resistance. Frontiers in Microbiology, 11. 


\subsection{Abstract}

Whole-genome sequencing (WGS) is used increasingly in public-health laboratories for typing and characterizing foodborne pathogens. To evaluate the performance of existing bioinformatic tools for in silico prediction of antimicrobial resistance (AMR) and serotypes of Salmonella enterica, WGS-based genotype predictions were compared with the results of traditional phenotyping assays. A total of 111 Salmonella enterica isolates recovered from a Canadian baseline study on broiler chicken conducted in 2012-2013 were selected based on phenotypic resistance to 15 different antibiotics and isolates were subjected to WGS. Both SeqSero2 and SISTR accurately determined Salmonella spp. Serotypes, with full matches to laboratory results for 86.5 And $88.3 \%$ of isolates, respectively. Antimicrobial resistance genes (ARGs) were identified using several bioinformatics tools including the Comprehensive Antibiotic Resistance Database - Resistance Gene Identifier (CARD-RGI), Center for Genomic Epidemiology (CGE) ResFinder web tool, short read sequence typer v 0.2.0 (SRST2), and $k$-mer alignment method (KMA v 1.17). All ARG identification tools had $\geq 99 \%$ accuracy for predicting resistance to all antibiotics tested except streptomycin (accuracy 94.6\%). Evaluation of ARG detection in assembled versus raw-read WGS data found minimal observable differences that were gene- and coverage- dependent. Where initial phenotypic results indicated isolates were sensitive, yet ARGs were detected, repeat AMR testing corrected discrepancies. All tools failed to find resistancedetermining genes for one gentamicin- and two streptomycin-resistant isolates. Further investigation found a single nucleotide polymorphism (SNP) in the nuoF coding region of one of the isolates which may be responsible for the observed streptomycin-resistant 
phenotype. Overall, WGS-based predictions of AMR and serotype were highly concordant with phenotype determination regardless of computational approach used.

\subsection{Introduction}

The overuse of antibiotics in hospitals, the community, and agriculture is believed to have accelerated the emergence of multi-drug resistant microorganisms (WHO, 2017a). This has resulted in increasing rates of antimicrobial resistance (AMR) globally posing a serious threat to public health. Without effective antibiotics to treat infectious diseases, healthcare costs, illness and mortality rates will rise. AMR surveillance programs provide data on the presence and emergence of AMR in the food production continuum (Dutil et al., 2010). In Canada, the Canadian Integrated Program for Antimicrobial Resistance Surveillance (CIPARS) monitors trends in antimicrobial use and resistance in selected bacterial organisms isolated from human, animal, and food sources across Canada (Government of Canada, 2007). Isolated organisms are tested for antibiotic susceptibility using phenotypic tests to determine the minimum inhibitory concentrations (MICs) of antimicrobials that are significant to public health.

A variety of AMR mechanisms have been characterized, including production of proteins or enzymes that inactivate or modify the antimicrobial, alteration of the antimicrobial target, reduced uptake, increased efflux, and overproduction of the target (Blair et al., 2015; Chan, 2016). Some bacteria are intrinsically resistant to certain antimicrobials through functional or structural characteristics (e.g. absence of target) (Blair et al., 2015). Alternatively, AMR can be acquired or developed through spontaneous mutation, horizontal gene transfer, and genetic recombination, all of which 
can provide a competitive advantage (Laxminarayan et al., 2013; Blair et al., 2015; Chan, 2016). Recent studies have identified a large number of genes responsible for intrinsic and/or acquired AMR in microorganisms (van Hoek et al., 2011; Blair et al., 2015).

The increasing affordability of whole genome sequencing (WGS) has resulted in the feasibility of whole genome-bacterial sequencing in clinical and food testing laboratories. Prediction of bacterial phenotypes based on WGS is convenient, rapid, and has many beneficial applications including use in outbreak investigations, diagnostics, and epidemiological surveillance (Zankari, 2014; Knowles et al., 2016; Edirmanasinghe et al., 2017; Carrillo et al., 2019). This has led to the development of a number of bioinformatic tools for predicting bacterial phenotypes, including AMR profiles and serotype (Zankari et al., 2012; McArthur et al., 2013; Gupta et al., 2014; Inouye et al., 2014; Zhang et al., 2015, 2019; Yoshida et al., 2016). WGS analysis for AMR has the advantage of providing the full complement of resistance genes present in an isolate as well as characterization of mutations that might confer resistance. Additional benefits include the ability to analyse a larger number of strains, as well as retrieve and re-analyse existing sequences, when new bioinformatics tools are developed and new genes are discovered, without time consuming culturing as is required for phenotypic testing.

There have been several investigations conducted to establish the concordance of AMR prediction based on detection of genetic markers and phenotypic resistance (Randall et al., 2004; Boerlin et al., 2005; Rosengren et al., 2009; Licker et al., 2015; Tyson et al., 2015, 2016; McDermott et al., 2016). WGS-based AMR prediction has been shown to be highly accurate for Salmonella and other organisms using custom AMR gene (ARG) databases (Tyson et al., 2015, 2016; McDermott et al., 2016; Zhao et al., 2016). 
Recognizing the need for common AMR prediction tools, a number of gene prediction databases are now available to the scientific community (Zankari et al., 2012; McArthur et al., 2013; Gupta et al., 2014; Inouye et al., 2014; Clausen et al., 2018; Feldgarden et al., 2019). However, a comprehensive comparison of multiple tools has not been reported.

Although surveillance studies have shown an increase in overall Salmonella antimicrobial resistance over time (Su et al., 2004), the resistance rate varies between different Salmonella serotypes, with different antimicrobials, and with variations in phage presence (Zhao et al., 2007; Hong et al., 2016; Yoon et al., 2017). Whereas clinical isolates of S. enterica ser. Typhimurium and S. enterica ser. Heidelberg from 2004-2012 were found to have the highest levels of clinically important resistance $(29.1$ and $24.8 \%$ respectively), analyses of veterinary Salmonella isolates in the United States from 2002 to 2003 found S. enterica ser. Uganda, S. enterica ser. Agona, and S. enterica ser. Newport commonly exhibited multidrug resistance (MDR) (Zhao et al., 2007). The correlation between AMR and certain Salmonella serotypes highlights the importance of monitoring and tracking in order to detect trends and inform policy for mitigating the impact of AMR.

Salmonella isolates are classified by serological reaction-based detection of somatic $\mathrm{O}$ antigens and phase variable flagellar $\mathrm{H}$ antigens $\mathrm{H} 1$ and $\mathrm{H} 2$ (Grimont and Weill, 2007; Zhang et al., 2015, 2019; Yoshida et al., 2016; Yachison et al., 2017). The combination or formula of expressed antigens is then used to identify a serotype based on the White-Kauffmann-Le Minor scheme (Grimont and Weill, 2007). As serology-based serotyping is expensive, labor intensive, and time consuming, molecular methods and 
bead-based array assays have been developed (Muñoz et al., 2010; Bopp et al., 2016;

Yoshida et al., 2016). Yet these techniques are still limited to identification of a portion of the approximately 2,500 Salmonella serotypes (Grimont and Weill, 2007; Zhang et al., 2015, 2019; Yoshida et al., 2016). WGS has the potential to allow rapid cost-effective identification of Salmonella isolates. The applications SeqSero and Salmonella in silico Typing Resource (SISTR) have recently been developed and evaluated for in silico determination of Salmonella serotypes using WGS data (Zhang et al., 2015, 2019; Yoshida et al., 2016; Yachison et al., 2017).

As the use of WGS-based analytical approaches for the characterization of bacterial pathogens to support public health investigations increases, it is critical to assess the reliability of tools developed for this purpose. This study provides a comparative analysis of the performance of publicly available bioinformatics tools to accurately predict serotype and antimicrobial resistance of 111 Salmonella isolated in Canada using assembled genomes and raw sequence reads. The sequence coverage requirements for the accurate detection of AMR are also investigated.

\subsection{Materials and Methods}

\subsubsection{Growth and maintenance of Salmonella strains}

The Salmonella spp. isolates $(\mathrm{n}=111)$ used in this study were selected from 2554 Salmonella collected between December 2012 and December 2013 by the Canadian Food Inspection Agency (CFIA) in collaboration with industry, federal and provincial partners as part of the national Microbiological Baseline Study (MBS) in Broiler Chickens 
(Government of Canada, 2016). Isolates were recovered in accordance with the Food Safety and Inspection Service (FSIS) method MLG 4.05 as described in detail in the MBS report (Government of Canada, 2016). Salmonella spp. isolates were submitted to the Public Health Agency of Canada (PHAC) - Salmonella Reference Laboratory in Guelph Ontario for serotyping and antimicrobial susceptibility testing. A total of 58 phenotypically resistant Salmonella and 53 phenotypically sensitive Salmonella, including 42 different serotypes, were selected for WGS based on differing resistance profiles (resistant to different antimicrobials in different combinations). Where possible an attempt was made to match a resistant strain with a sensitive strain of the same serotype. All strains were stored at $-80^{\circ} \mathrm{C}$ in $25 \%$ glycerol and were plated on Brain-Heart Infusion agar (BHI) (Oxoid, Nepean, ON) and incubated overnight (14-16 hours) at $37^{\circ} \mathrm{C}$ prior to use.

\subsubsection{Traditional Serotyping and Antimicrobial susceptibility testing}

All strains used in this study were previously serotyped using traditional methods at the PHAC Salmonella Reference Laboratory (Guelph, Ontario). Standard methods were used to determine antigenic formula of each strain (Shipp and Rowe, 1980), and serotypes were assigned based on the White-Kauffmann-Le Minor scheme (Grimont and Weill, 2007).

Strains had also been previously tested for antimicrobial resistance by means of the broth microdilution method using the Sensititre Vizion ${ }^{\mathrm{TM}}$ automated system (Trek Diagnostic Systems, Cleveland, $\mathrm{OH}$ ) at PHAC as described by the Canadian Integrated Program for Antimicrobial Resistance Surveillance (CIPARS) (Government of Canada, 
2013). Briefly, the CMV2AGNF plate was used to test for resistance to 15

antimicrobials: gentamicin, GEN; kanamycin, KAN; streptomycin, STR; amoxicillinclavulanic acid, AMC; cefoxitin, FOX; ceftiofur, TIO; ceftriaxone, CRO; ampicillin, AMP; chloramphenicol, CHL; sulfisoxazole, SOX; trimethoprim-sulfamethoxazole, SXT; tetracycline, TCY; nalidixic acid, NAL; and ciprofloxacin, CIP. Isolates were streaked on Mueller Hinton (MH) or MacConkey agar and incubated at $36^{\circ} \mathrm{C}$ for 18 to 24 hours. One colony was selected from each plate, re-streaked for purification, and incubated; a 0.5 -McFarland suspension was prepared by transferring growth from the agar plates to $5.0 \mathrm{~mL}$ of sterile, demineralized water. Ten microliters of suspension were transferred to $10 \mathrm{~mL}$ of $\mathrm{MH}$ broth (MHB) and dispensed onto CMV2AGNF testing plates at $50 \mu \mathrm{L}$ per well and sealed. Plates were read automatically with the plate reading system after $18 \mathrm{hrs}$ incubation at $36^{\circ} \mathrm{C}$. Breakpoints for resistance determination were determined according to CLSI guidelines M100-S23 and M31-A3 unless stated otherwise (Clinical and Laboratory Standards Institute, 2008, 2013).

\subsection{3 gDNA isolation and whole-genome sequencing (WGS) of selected Salmonella}

For each isolate a single colony was transferred from BHI agar to $800 \mu \mathrm{L}$ of BHI broth (Oxoid, Ottawa, Canada) and incubated at $37^{\circ} \mathrm{C}$ for $3 \mathrm{~h}$ following which genomic DNA was isolated from $400 \mu \mathrm{L}$ of broth culture using the Promega Maxwell@ 16 Cell DNA purification kit (Promega, Madison, WI). Double-stranded genomic DNA was quantified using the Quant-iT TM High Sensitivity Assay kit (Life Technologies Inc., Burlington, ON) according to the manufacturers' recommendations. Sequencing libraries were constructed using the Nextera XT DNA sample preparation and the Nextera XT 
Index Kits (Illumina, Inc., San Diego, CA) and paired-end sequencing was performed on the Illumina MiSeq platform, using 600-cycle MiSeq reagent kits (v3) with 5\% PhiX control (Illumina Inc.).

\subsubsection{Bioinformatic analysis}

Quality of raw sequencing reads was assessed with FastQC version 0.11.8 (Andrews, 2010). Quality trimming was performed with BBDuk from BBTools version 38.22 (Bushnell, 2014) with the following parameters: trim quality of 10 and removal of reads below 50 bp long. Error correction was performed using tadpole version 8.22 (Bushnell, 2014) in 'correct' mode with default parameters. Sequences were checked for contamination using ConFindr 0.5.0 with default parameters (Low et al., 2019). Contigs were assembled from the trimmed and error-corrected reads using SKESA version 2.3.0 with the vector percent argument disabled (Souvorov et al., 2018). For assembled versus raw-read analyses where SPAdes assemblies were used, the same trimming and error correction steps were performed, and assemblies were created using SPAdes version 3.12.0 on default settings with the -only-assembler option (Bankevich et al., 2012). Pilon version 1.22 (Walker et al., 2014) was used to perform one round of automatic assembly improvement, and quality was assessed with Qualimap version 2.2.2 (García-Alcalde et al., 2012; Okonechnikov et al., 2016). A targeted minimum sequence coverage of 20X and minimum Phred quality score of 10 was used for sequence data. Plasmids were predicted and reconstructed from assembled genomes using the MOB-recon tool from MOB-suite v 1.4.1 (Robertson and Nash, 2018). 
Serotyping of Salmonella spp. in silico was conducted using both raw reads and assembled genomes with SeqSero version 2 (SeqSero2), and with assemblies using the Salmonella In Silico Typing Resource (SISTR) developed by Zhang et al. $(2015,2019)$ and Yoshida et al. (2016), respectively. SISTR "overall" serovar predictions were used, as described by Yoshida et al. (2016). Analysis of separated paired end raw reads with SeqSero2 was conducted using both raw reads allele micro-assembly mode and k-mer mode, while assemblies were analysed using the k-mer mode. Serotype predictions were compared to laboratory results and results were interpreted according to categories described by Yachison et al. (2017). Briefly, matches that were concordant with laboratory results were categorized as "full". In cases where multiple serotypes were predicted (including the laboratory result), matches were categorized as "inconclusive", and in cases where results differed because one or more of the antigen genes were not expressed and therefore not detected by laboratory methods, results were categorized as "incongruent". Results were considered "incorrect" in cases where serovar predictions were different from the laboratory results.

Single nucleotide variant (SNV) analysis of phenotypically resistant strains OLC2536, OLC2644, and OLC2626 with closely related sensitive strains was conducted using the Single Nucleotide Variant PHYLogenomics (SNVPhyl) pipeline version 1.0.1 (Petkau et al., 2017) with the reference set as the sensitive strain. High-quality SNVs had a minimum coverage of 5 reads, with $75 \%$ of reads supporting the SNV identification, and a filter density window of 500 with a density threshold of 2 . 


\subsubsection{ARG identification in WGSs}

Resistance genes were identified using each of the tools described in Table 2.1. The CARD-RGI tool was installed using bioconda from https://card.mcmaster.ca/download (Grüning et al., 2018). CGE’s PointFinder and ResFinder v2.1 web tools with default settings, threshold for \%ID 90\% and minimum length $60 \%$, were used for analyses. The NCBI Antimicrobial Resistance Reference Gene Database (Bioproject PRJNA313047) (NCBI-AMR db) was downloaded from NCBI on May 29, 2018. The ARG-Annot and ResFinder databases for use with SRST2 (v 0.2.0) were downloaded from the SRST2 github (https://github.com/katholt/srst2). The ResFinder database for use with KMA was installed via bitbucket with the KMA v1.0 tool as per author's instructions. For all tools, ARGs were identified using a minimum cutoff of $90 \%$ nucleotide identity over a minimum length of $60 \%$ except for investigations of genotype-phenotype discrepancies where the select minimum length was lowered from $60 \%$ to $40 \%$, and where stated otherwise. 
Table 2.1. Characteristics of ARG detection tools.

\begin{tabular}{|c|c|c|c|c|c|}
\hline Tool & Database $^{a}$ & $\begin{array}{l}\text { Last Update } \\
\text { prior this } \\
\text { publication }^{\text {b }}\end{array}$ & $\begin{array}{l}\text { Supported } \\
\text { Sequence } \\
\text { Format }\end{array}$ & Originator & Reference \\
\hline $\begin{array}{l}\text { ResFinder v2. } \\
\text { (web tool) }\end{array}$ & & 2019 & Fasta & CGE & $\begin{array}{l}\text { Zankari et al., } \\
2012\end{array}$ \\
\hline $\begin{array}{l}\text { KMA } \\
\text { v1.17 }\end{array}$ & $\begin{array}{l}\text { ResFinder } \\
\text { NCBI }\end{array}$ & $\begin{array}{l}2018 \\
2018\end{array}$ & Fasta, Fastq & CGE & $\begin{array}{l}\text { Clausen et al., } \\
2018\end{array}$ \\
\hline SRST2 & $\begin{array}{l}\text { ResFinder } \\
\text { ARG-Annot } \\
\text { NCBI }\end{array}$ & $\begin{array}{l}2014 \\
2016-07 \\
2018\end{array}$ & Fastq & $\begin{array}{l}\text { University of } \\
\text { Melbourne }\end{array}$ & $\begin{array}{l}\text { Gupta et al., } \\
\text { 2014; Inouye et } \\
\text { al., } 2014\end{array}$ \\
\hline CARD-RGI & & 2019 & Fasta & $\begin{array}{l}\text { McMaster } \\
\text { University }\end{array}$ & $\begin{array}{l}\text { McArthur et al. } \\
2013\end{array}$ \\
\hline
\end{tabular}

Abbreviations: ARG, antimicrobial resistance gene; ResFinder, Resistance Finder; CGE, Center for Genomic Epidemiology; KMA, $k$-mer alignment method; SRST2, short read sequence typer v.0.2.0; ARG-Annot, antimicrobial resistance gene annotation; NCBI, National Center for Biotechnology Information Bacterial Antimicrobial Resistance Reference Gene Database; CARD-RGI, comprehensive Antibiotic Resistance Database Resistance Gene Identifier

${ }^{\text {a }}$ Database(s) used with tool (if multiple options were possible).

${ }^{\mathrm{b}}$ Year provided is for the database version used in this study

For chromosomal structural gene and single nucleotide variant (SNV) mutations CGE's PointFinder program for identifying chromosomal mutations (now part of ResFinder) was used to investigate known mutations, while BLAST was used to investigate genes where SNVs were found in resistant strains compared to sensitive strains, and possibly conferred resistance (Camacho et al., 2009; Zankari et al., 2017). Sequences with $<100 \%$ amino acid identity to the DNA gyrase subunit A gene (gyrA) were reviewed to determine whether they matched known nalidixic acid-resistant (NalR) mutations (Table 2.2). This analysis was also conducted for the 111 Salmonella strains to search for mutations in aroD, $c y o B, c y o C, f u s A, \operatorname{gln} A, \operatorname{gid} B$, isp $A, n u o E, n u o F, \operatorname{prf} B$, rpsL, trkH, $u b i A, u b i E$, and $u b i F$ genes which have a reported role in conversion of 
Salmonella to a small colony variant (SCV) phenotype and/or confer streptomycin resistance (Soballe and Poole, 1999; Springer et al., 2001; Cano et al., 2003; Okamoto et al., 2007; Koskiniemi et al., 2011; Lázár et al., 2013; Li et al., 2016; Aurass et al., 2017).

Table 2.2. Identification of single nucleotide variations resulting in non-synonymous mutations.

\begin{tabular}{|c|c|c|c|c|c|c|}
\hline Gene & Isolate & $\begin{array}{l}\text { Nucleotide } \\
\text { Identity } \\
(\%)\end{array}$ & $\begin{array}{l}\text { Amino } \\
\text { Acid } \\
\text { Identity } \\
(\%) \\
\end{array}$ & Mutation & $\begin{array}{l}\text { Mutation } \\
\text { Type }^{\mathrm{a}}\end{array}$ & $\begin{array}{l}\text { Product or } \\
\text { Function }\end{array}$ \\
\hline \multirow{2}{*}{ gyrA } & OLC2588 & 99.73 & 99.17 & 83- TCC (Ser) $\rightarrow$ TTC (Phe) & $\mathrm{NC}$ & \multirow{2}{*}{$\begin{array}{l}\text { DNA gyrase } \\
\text { subunit A }\end{array}$} \\
\hline & OLC2622 & 99.73 & 99.17 & 83- TCC (Ser) $\rightarrow$ TTC (Phe) & $\mathrm{NC}$ & \\
\hline \multirow{4}{*}{ nиоF } & OLC2536 & 99.93 & 99.78 & 45- CTG (Leu) $\rightarrow$ CGC (Arg) & $\mathrm{SC}$ & \multirow{4}{*}{$\begin{array}{l}\text { NADH: } \\
\text { ubiquinone } \\
\text { oxidoreductase } \\
\text { (subunit F: the } \\
\text { binding site) }\end{array}$} \\
\hline & OLC2556 & 98.51 & 99.78 & 378- CCG (Pro) $\rightarrow$ TCG (Ser) & $\mathrm{SC}$ & \\
\hline & OLC2562 & 98.43 & 99.78 & 257- AAG (Lys) $\rightarrow$ AGG (Arg) & $\mathrm{C}$ & \\
\hline & OLC2563 & 98.43 & 99.78 & 257- AAG (Lys) $\rightarrow$ AGG (Arg) & $\mathrm{C}$ & \\
\hline \multirow{2}{*}{$\operatorname{prfB}$} & OLC2619 & 98.87 & 99.66 & 93- GTC (Val) $\rightarrow$ ATC (Ile) & $\mathrm{C}$ & \multirow{2}{*}{$\begin{array}{l}\text { Peptide chain } \\
\text { release factor } 2\end{array}$} \\
\hline & OLC2642 & 98.87 & 99.66 & 93- GTC (Val) $\rightarrow$ ATC (Ile) & $\mathrm{C}$ & \\
\hline \multirow{6}{*}{ ubiA } & OLC2587 & 99.31 & 99.66 & 242- GCT (Ala) $\rightarrow$ TCT (Ser) & $\mathrm{C}$ & \multirow{6}{*}{$\begin{array}{l}\text { Ubiquinone } \\
\text { biosynthesis } \\
\text { Parahydroxyben- } \\
\text { zoate } \\
\text { ocatprenyltransf- } \\
\text { erase }\end{array}$} \\
\hline & OLC2591 & 99.2 & 99.66 & 221- GGC (Gly) $\rightarrow$ GCC (Ala) & $\mathrm{SC}$ & \\
\hline & OLC2593 & 99.2 & 99.66 & 41- CCG (Pro) $\rightarrow$ TCG (Ser) & $\mathrm{NC}$ & \\
\hline & OLC2612 & 99.2 & 99.66 & 41- CCG (Pro) $\rightarrow$ TCG (Ser) & $\mathrm{NC}$ & \\
\hline & OLC2613 & 99.2 & 99.66 & 41- CCG (Pro) $\rightarrow$ TCG (Ser) & $\mathrm{NC}$ & \\
\hline & OLC2625 & 99.31 & 99.66 & 220-CTT (Leu) $\rightarrow$ TTT(Phe) & $\mathrm{C}$ & \\
\hline
\end{tabular}

\footnotetext{
${ }^{a}$ Refers to the type of amino acid substitution: NC, non-conservative, residues are not similar; SC, semiconservative, residues have weakly similar properties; C, conservative, residues have strongly similar properties.
}

The performance of AMR detection tools was evaluated by assessing the accuracy of WGS-based predictions relative to the Sensititre Vizion ${ }^{\mathrm{TM}}$ phenotype results for each antibiotic. A true positive (TP) was defined as a result where the WGS analysis of an 
isolate predicted a resistance gene and the strain displayed a resistant phenotype. A false positive (FP) was defined as a result where WGS analysis predicted a resistance gene but the isolate was phenotypically sensitive. A true negative (TN) was defined as a result where WGS analysis predicted no ARGs and the isolate was phenotypically sensitive. A false negative (FN) was defined as a result where WGS analysis did not detect an ARG but the isolate was phenotypically resistant. The accuracy of each tool for each antibiotic was calculated by dividing the sum of TP and TN by the total population $(n=111)$ and multiplied by 100 . The overall accuracy for each tool was determined by dividing the sum of TP and TN for all resistances combined divided by the combined number of predictions $(\mathrm{n}=1332)$.

\subsubsection{Nucleotide sequence accession numbers}

Whole-genome sequences have been deposited at DDBJ/EMBL/GenBank in bioproject PRJNA417863. Sequence read archive (SRA) accession numbers and phenotype data are listed in supplementary Table S2.1.

\subsubsection{Raw read sampling to determine minimum coverage requirements for ARG detection}

To determine the minimum genome coverage required for accurate ARG detection, the raw reads for each isolate were randomly subsampled to coverage levels of $1 \mathrm{X}, 2.5 \mathrm{X}, 5 \mathrm{X}, 10 \mathrm{X}, 15 \mathrm{X}$ and $20 \mathrm{X}$ (100 replicates per isolate at each coverage level) using the reformat.sh script (version 37.61) provided with the BBMap suite (Bushnell, 2014). Subsampled reads were analysed for the presence of AMR genes using the $k$-mer 
alignment method (KMA v 1.17) (Clausen et al., 2018) with the NCBI-AMR db and default settings. For each isolate, 100 replicates were sampled at each coverage level $(\mathrm{n}=$ 111 isolates, six coverage levels).

\subsubsection{Analysis of ARG-detection in assembled versus raw-read sequences}

Additional subsampling was conducted in order to test the effects of assembly on ARG detection. Raw reads for a subset of seven isolates (Table 2.3) were randomly subsampled to levels of $5 \mathrm{X}, 10 \mathrm{X}, 15 \mathrm{X}$, and 20X coverage as described above (20 replicates per isolate at each coverage level). For each isolate, all replicates at each coverage level were then assembled as described above. ARG detection was conducted using KMA v1.17 with the NCBI-AMR db and default values for both assembled and raw-read sequences. To evaluate statistical significance, comparison of gene-detection in assembled and raw-read sequences was conducted for each gene at each coverage level using the Fisher's exact test in R version 3.6.1(R Core Team, 2014).

Table 2.3. ARG profiles and locations in subset of $S$. enterica subspecies enterica isolates used for assembly versus raw-read analyses.

\begin{tabular}{|c|c|c|c|}
\hline Isolate & Location & Plasmid Inc Type $^{a}$ & ARG(s) ${ }^{b}$ \\
\hline \multirow[t]{2}{*}{ OLC2545 } & Chr & & aac(6')-Iaa \\
\hline & Pmd 960 & $\mathrm{Inc} \mathrm{A} / \mathrm{C} 2$ & 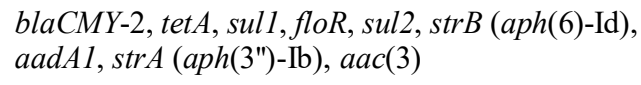 \\
\hline \multirow[t]{4}{*}{ OLC2552 } & Chr & & fos $A 7, \operatorname{aac}\left(6^{\prime}\right)$-Iaa \\
\hline & Pmd 53 & IncX1 & - \\
\hline & Pmd 95 & ColRNAI_rep_cluster_1987 & - \\
\hline & Pmd 292 & ColRNAI_rep_cluster_1857 & - \\
\hline \multirow[t]{3}{*}{ OLC2564 } & $\mathrm{Chr}$ & & fos $A 7, \operatorname{aac}\left(6^{\prime}\right)$-Iaa \\
\hline & Pmd 473 & IncI1 & sull, aac(3), aadA1 \\
\hline & Pmd 695 & & tet $A$ \\
\hline
\end{tabular}




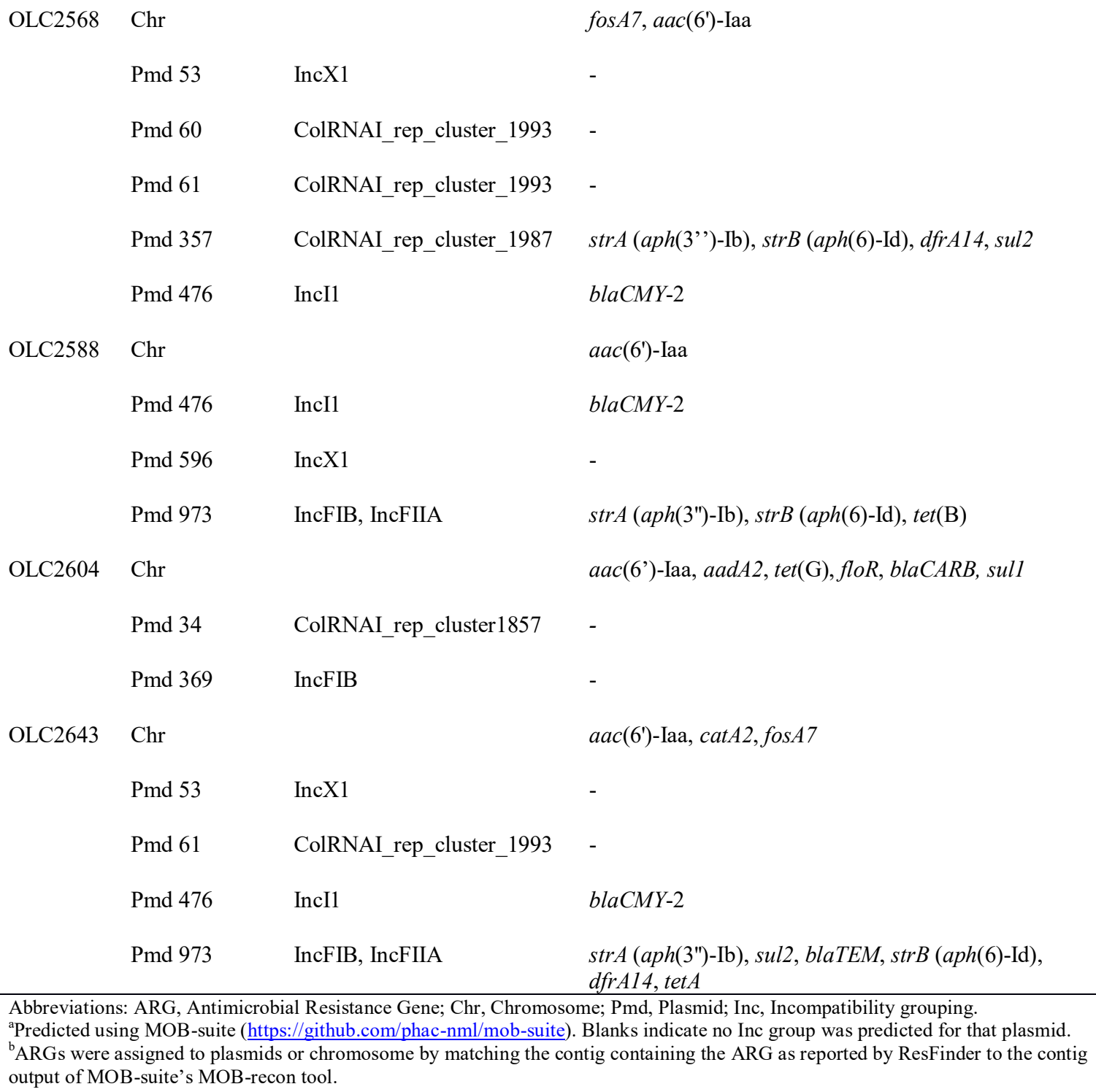

\subsubsection{Resistance phenotype verification via broth microdilution}

Discrepancies observed between original AMR genotypes and phenotypes were retested using the broth microdilution method as described by Wiegand et al. (2008). Eleven strains including 4 strains with genotypic resistance and phenotypic sensitivity, 4 control strains with genotypic and phenotypic resistance, 2 strains with a sensitive genotype and phenotypic resistance, and the type strain ATCC 25922 Escherichia coli 
(sensitive control) were tested in sterile 96-well microtiter plates. Antimicrobial concentrations tested included GEN $(0.25-16 \mu \mathrm{g} / \mathrm{ml})$, FOX $(0.5-32 \mu \mathrm{g} / \mathrm{ml})$, AMC (1/0.5 - 32/16 $\mu \mathrm{g} / \mathrm{ml})$, TCY $(1-64 \mu \mathrm{g} / \mathrm{ml})$, and STR $(2-128 \mu \mathrm{g} / \mathrm{ml})$. Uninoculated MHB (Oxoid, Nepean, ON) wells were included as a contamination control. Each of the 11 isolates was inoculated at concentration of approximately $5 \times 10^{5} \mathrm{CFU} / \mathrm{mL}$ and incubated at $37^{\circ} \mathrm{C}$ for $24 \mathrm{~h}$. All strains were tested for all antibiotics.

\subsubsection{Streptomycin sensitivity via agar dilution}

STR phenotypic resistance was re-evaluated using the agar dilution method using protocols adapted from Wiegand et al. (2008). Briefly, isolates were streaked for single colonies onto MH agar (MHA) and incubated overnight at $37^{\circ} \mathrm{C}$. STR was diluted in MHA at concentrations of $0,2,4,8,16,32$, and $64 \mu \mathrm{g} / \mathrm{mL}$. A 0.5 McFarland suspension of each isolate was made then diluted 1:10 in MHB. A 48-pin replicator was used to spot $1 \mu \mathrm{L}$ aliquots on dried MHA containing STR in duplicate moving from lowest to highest STR concentration in duplicate $(0 \mu \mathrm{g} / \mathrm{mL}$ being first as a viability control). E. coli ATCC 25922 was included as a sensitive control. All plates were incubated overnight at $37^{\circ} \mathrm{C}$.

The MIC for each isolate was recorded as the lowest concentration of STR that completely inhibited growth.

\subsubsection{Activation of Cryptic Aminoglycoside Resistance in Minimal Media}

To further investigate possible resistance mechanisms for 16 isolates that were resistant to STR, but with no identified ARGs, MICs for STR were evaluated using a method adapted from Koskiniemi et al. (2011). Briefly, MH (Oxoid, Nepean, ON), Luria- 
Bertani - Lennox (LB) (Sigma-Aldrich, Oakville, ON), and M9 minimal salts 5X powder (Sigma-Aldrich, Oakville, ON) supplemented with $2 \mathrm{mM} \mathrm{MgSO}_{4}, 0.1 \mathrm{mM} \mathrm{CaCl}_{2}$, and $0.4 \%$ glucose were prepared in both broth and agar forms. Agars contained 0, 2, 4, 8, 16, 32, 64 , or $128 \mu \mathrm{g} / \mathrm{ml}$ STR. A $0.5 \mathrm{McFarland}$ suspension was prepared for each isolate in 0.9\% saline using a fresh overnight culture, including E. coli ATCC 25922 as a control.

For broth microdilution testing using the media described above, the CMV4AGNF Sensititre plate (Trek Diagnostic Systems, Cleveland, OH; Thermo Fisher Scientific, USA) was used to test for resistance to streptomycin (STR) at concentrations of $2,4,8,16,32$, and $64 \mu \mathrm{g} / \mathrm{mL}$. A $1 \%$ stock of TTC (2,3,5-triphenyl-tetrazolium chloride) (Sigma-Aldrich, Oakville, ON) was mixed with each broth type (M9B, MHB, LB) to create a $0.005 \%$ TTC-broth solution. McFarland suspensions were then diluted 1:1000 in each $0.005 \%$ TTC-broth type, vortexed, and distributed into the wells of a CMV4AGNF Sensititre plate.

For agar dilution testing, 0.5 McFarland suspensions were diluted 1:10 in $0.9 \%$ saline and $2 \mu 1$ was spotted onto each agar type in duplicate as described above. All agar and broth microtiter plates were incubated at $37^{\circ} \mathrm{C}$ for 20 hours. MIC for each mediaantimicrobial combination was recorded as the lowest concentration of antibiotic that led to complete growth inhibition. 


\subsection{Results}

\subsubsection{Determining Salmonella spp. Serotypes in silico}

Both SeqSero and SISTR correctly identified most of the isolates, and no incorrect serotype predictions were observed (Table 2.4). For SeqSero2 used with either raw reads or assembled genomes, full matches were observed for 96 serotype predictions $(86.5 \%)$. SISTR was slightly more accurate, with full matches observed for $98(88.3 \%)$ of the isolates tested. No incorrect results were observed in the dataset used in this study. Neither tool was able to accurately predict the serotype Othmarschen, however SISTR did report these three isolates as "Haelsingborg|Moers|Oranienburg|Othmarschen" (inconclusive) whereas SeqSero identified these as the closely related Oranienberg serovar. In the latter case results were classified as inconclusive rather than incorrect due to the close relationship of these serovars (Robertson et. al, 2018). SeqSero2 generated inconclusive results for Albany and Molade whereas SISTR was able to accurately assign these serovars (Table 2.5). Incongruent matches were observed for 9 SeqSero and SISTR predictions (Table 2.4). For four of the strains identified as serovar Kentucky by both SISTR and SeqSero (OL2571, OLC2572, OLC2573, OLC2621) only some of the antigens were expressed based on traditional serotyping results, even though genes encoding the antigens were detected in the genomes (Table S2.1). A similar situation was observed for a Hadar isolate (OLC2574), a Mbandaka isolate (OLC2641) and 2 Senftenberg isolates (OLC2640), (OLC2616). One I:Rough-O:R:1,5 isolate (OLC2582), was identified as serovar Infantis by SISTR; however, genes encoding the O-antigen were not identified by SeqSero (Table S2.1). Finally, one of the monophasic variants of 
Salmonella Typhimurium (OLC2556) was identified as Typhimurium (Table 2.5).

Discrepancies between serotype prediction and conventional serotyping were not evaluated by repeating the serotyping.

Table 2.4. Performance of in silico tools for detecting Salmonella serotype

\begin{tabular}{lccc}
\hline Match Result & SeqSero2 Assembled & SeqSero2 Raw Reads & SISTR \\
\hline Full & $96(86.5 \%)$ & $96(86.5 \%)$ & $98(88.3 \%)$ \\
Inconclusive & $5(4.5 \%)$ & $5(4.5 \%)$ & $3(2.7 \%)$ \\
Incongruent & $10(9.0 \%)$ & $10(9.0 \%)$ & $10(9.0 \%)$ \\
\hline Total & 111 & 111 & 111
\end{tabular}

Abbreviations: SeqSero, sequence serotyping tool; SISTR, Salmonella in silico typing resource tool. Match Result definitions: Full, serotype prediction concordant with laboratory method; Inconclusive, multiple serotypes were predicted including the laboratory result; Incongruent, results differed because one or more of the antigen genes were not expressed and therefore not detected by laboratory methods.

\subsubsection{Antimicrobial Resistance: Relationship of Phenotype and Genotype}

Four ARG detection tools and four ARG databases were used in seven different combinations (Table 2.1) to identify at total of 178 ARGs in the 111 Salmonella isolates included in this study. With only two exceptions, the ARG tools generated equivalent results (Table 2.6). KMA analysis of isolate assemblies failed to detect the $d f r A 15$ gene for trimethoprim resistance in two isolates using the ResFinder database supplied with the tool. However, KMA analysis of these isolates using the ResFinder database with rawreads, as well as the NCBI database with assemblies, accurately detected the $d f r A 15$ gene. The ResFinder tool failed to detect the sul 1 gene for resistance to SOX in a single isolate 
resulting in $99.1 \%$ predictive accuracy. However, the tool was able to detect sul1 when the select minimum length was lowered to $40 \%$. Further examination revealed that the gene was split between two contigs. This analysis has since been repeated with ResFinder version 3.1 where the gene is reported as a $>99 \%$ identity for $535 / 867$ bases of Query/Template Length. Further analysis found the sull gene was split over two contigs in this assembly. 
Table 2.5. Predicting Salmonella serotypes using WGS data

\begin{tabular}{|c|c|c|c|c|c|c|c|c|c|c|c|}
\hline \multirow{2}{*}{ Group } & \multirow{2}{*}{ Serotype $^{a}$} & \multirow{2}{*}{$\begin{array}{l}\text { Subspecie } \\
\text { s }\end{array}$} & \multirow{2}{*}{$\begin{array}{l}\text { Somatic }(O) \\
\text { antigens }^{b}\end{array}$} & \multicolumn{3}{|c|}{ Flagellar $(\mathrm{H})$ antigens } & \multirow[t]{2}{*}{ No. of Isolates } & \multicolumn{2}{|c|}{ SISTR ${ }^{*}$} & \multicolumn{2}{|c|}{ SeqSero } \\
\hline & & & & Phase 1 & Phase 2 & Other $^{\mathrm{c}}$ & & $\overline{T P}$ & FP & TP & FP \\
\hline \multirow[t]{3}{*}{$\bar{O}: 1,3,19\left(\mathrm{E}_{4}\right)$} & Senftenberg & $\mathrm{I}$ & $1,3,19$ & $\mathrm{~g},[\mathrm{~s}], \mathrm{t}$ & - & $\begin{array}{l}{\left[\mathrm{z}_{27}\right],\left[\mathrm{z}_{34}\right],\left[\mathrm{z}_{37}\right]} \\
{\left[\mathrm{z}_{43}\right],\left[\mathrm{z}_{45}\right],\left[\mathrm{z}_{46}\right]} \\
{\left[\mathrm{z}_{82}\right]}\end{array}$ & 1 & 1 & 2 & 1 & 2 \\
\hline & I:Rough-O:g,s,t:- & I & Rough & $\mathrm{g}, \mathrm{s}, \mathrm{t}$ & - & & 1 & 0 & 0 & 0 & 0 \\
\hline & I:19:-:- & $\mathrm{I}$ & 19 & - & - & & 1 & 0 & 0 & 0 & 0 \\
\hline \multirow[t]{2}{*}{$\mathrm{O}: 3,10\left(\mathrm{E}_{1}\right)$} & Anatum & $\mathrm{I}$ & $3,\{10\}\{\underline{15}\}\{\underline{15}, \underline{34}\}$ & $\mathrm{e}, \mathrm{h}$ & 1,6 & {$\left[\mathrm{z}_{64}\right]$} & 1 & 1 & 0 & 1 & 0 \\
\hline & Orion & I & $3,\{10\}\{\underline{15}\}\{\underline{15}, \underline{34}\}$ & $\mathrm{y}$ & 1,5 & & 1 & 1 & 0 & 1 & 0 \\
\hline \multirow[t]{7}{*}{$\mathrm{O}: 4(\mathrm{~B})$} & Agona & $\mathrm{I}$ & $\underline{1}, 4,[5], 12$ & $\mathrm{f}, \mathrm{g}, \mathrm{s}$ & {$[1,2]$} & {$\left[\mathrm{z}_{27}\right],\left[\mathrm{z}_{45}\right]$} & 2 & 2 & 0 & 2 & 0 \\
\hline & Heidelberg & I & $\underline{1}, 4,[5], 12$ & $\mathrm{r}$ & 1,2 & & 19 & 19 & 0 & 19 & 0 \\
\hline & Kiambu & $\mathrm{I}$ & $\underline{1}, 4,12$ & $\mathrm{z}$ & 1,5 & & 5 & 5 & 0 & 5 & 0 \\
\hline & Saintpaul & I & $\underline{1}, 4,[5], 12$ & $\mathrm{e}, \mathrm{h}$ & 1,2 & & 1 & 1 & 0 & 1 & 0 \\
\hline & Schwarzengrund & I & $\underline{1}, 4,12,27$ & $\mathrm{~d}$ & 1,7 & & 5 & 5 & 0 & 5 & 0 \\
\hline & Typhimurium & $\mathrm{I}$ & $\underline{1}, 4,[5], 12$ & $\mathrm{i}$ & 1,2 & & 12 & 12 & 1 & 12 & 1 \\
\hline & I:4,5,12:i:- & $\mathrm{I}$ & $4,5,12$ & i & - & & 4 & 3 & 0 & 3 & 0 \\
\hline \multirow[t]{10}{*}{$\mathrm{O}: 7\left(\mathrm{C}_{1}\right)$} & Braenderup & I & $6,7, \underline{14}$ & $\mathrm{e}, \mathrm{h}$ & $\mathrm{e}, \mathrm{n}, \mathrm{Z}_{15}$ & & 2 & 2 & 0 & 2 & 0 \\
\hline & Infantis & I & $6,7, \underline{14}$ & $\mathrm{r}$ & 1,5 & $\begin{array}{l}{[\mathrm{R} 1 \ldots],\left[\mathrm{z}_{37}\right],\left[\mathrm{z}_{45}\right]} \\
{\left[\mathrm{z}_{49}\right]}\end{array}$ & 6 & 6 & 1 & 6 & $0^{\mathrm{c}}$ \\
\hline & I:Rough-O:r:1,5 & $\mathrm{I}$ & Rough & $\mathrm{r}$ & 1,5 & & 1 & 0 & 0 & 0 & 0 \\
\hline & Mbandaka & I & $6,7, \underline{14}$ & $\mathrm{z}_{10}$ & e,n,z15 & {$\left[\mathrm{z}_{37}\right],\left[\mathrm{z}_{45}\right]$} & 3 & 3 & 1 & 3 & 1 \\
\hline & I:6,7:-:- & $\mathrm{I}$ & 6,7 & - & - & & 1 & 0 & 0 & 0 & 0 \\
\hline & Montevideo & $\mathrm{I}$ & $6,7, \underline{14}$ & $\mathrm{~g}, \mathrm{~m},[\mathrm{p}], \mathrm{s}$ & {$[1,2,7]$} & & 1 & 1 & 0 & 1 & 0 \\
\hline & Ohio & I & $6,7, \overline{14}$ & $\mathrm{~b}$ & $1, \mathrm{w}$ & {$\left[\mathrm{Z}_{59}\right]$} & 3 & 3 & 0 & 3 & 0 \\
\hline & Othmarschen & I & $6,7, \underline{14}$ & $\mathrm{~g}, \mathrm{~m},[\mathrm{t}]$ & - & & 3 & $3-\mathrm{I}$ & 0 & 0 & 0 \\
\hline & Oranienburg & $\mathrm{I}$ & $6,7, \underline{14}$ & $\mathrm{~m}, \mathrm{t}$ & {$\left[\mathrm{z}_{57}\right]$} & & 0 & 0 & 0 & 0 & 3 \\
\hline & Tennessee & I & $6,7, \underline{14}$ & $\mathrm{z}_{29}$ & {$[1,2,7]$} & & 1 & 1 & 0 & 1 & 0 \\
\hline \multirow{12}{*}{$\mathrm{O}: 8\left(\mathrm{C}_{2}-\mathrm{C}_{3}\right)$} & Thompson & I & $6,7, \underline{14}$ & $\mathrm{k}$ & 1,5 & {$[\mathrm{R} 1 \ldots]$} & 4 & 4 & 0 & 4 & 0 \\
\hline & Albany & $\mathrm{I}$ & $8, \underline{20}$ & $\mathrm{z}_{4}, \mathrm{z}_{24}$ & - & {$\left[\mathrm{z}_{45}\right]$} & 1 & 1 & 0 & $1-\mathrm{I}$ & 0 \\
\hline & Hadar & I & 6,8 & $\mathrm{z}_{10}$ & $e, n, x$ & & 4 & 4 & 1 & 4 & 1 \\
\hline & $\begin{array}{l}\text { I:Rough- } \\
O: z_{10}: e, n, x\end{array}$ & I & Rough & $\mathrm{z}_{10}$ & $e, n, x$ & & 1 & 0 & 0 & 0 & 0 \\
\hline & Kentucky & I & $8, \underline{20}$ & $\mathrm{i}$ & $\mathrm{z}_{6}$ & & 9 & 9 & 4 & 9 & 4 \\
\hline & I:8,20:-:- & $\mathrm{I}$ & 8,20 & - & - & & 1 & 0 & 0 & 0 & 0 \\
\hline & I:8,20:I:- & $\mathrm{I}$ & 8,20 & $\mathrm{i}$ & - & & 1 & 0 & 0 & 0 & 0 \\
\hline & $\mathrm{I}: 8,20:-: \mathrm{z}_{6}$ & $\mathrm{I}$ & 8,20 & - & $\mathrm{z}_{6}$ & & 1 & 0 & 0 & 0 & 0 \\
\hline & I:Rough-O:i: $z_{6}$ & I & Rough & i & $\mathrm{z}_{6}$ & & 1 & 0 & 0 & 0 & 0 \\
\hline & Litchfield & $\mathrm{I}$ & 6,8 & $1, \mathrm{v}$ & 1,2 & & 2 & 2 & 0 & 2 & 0 \\
\hline & Molade & I & $8, \underline{20}$ & $\mathrm{z}_{10}$ & $\mathrm{z}_{6}$ & & 1 & 1 & 0 & $1-\mathrm{I}$ & 0 \\
\hline & Muenchen & I & 6,8 & $\mathrm{~d}$ & 1,2 & {$\left[\mathrm{z}_{67}\right]$} & 2 & 2 & 0 & 2 & 0 \\
\hline $\mathrm{O}: 9\left(\mathrm{D}_{1}\right)$ & Enteritidis & I & $\underline{1}, 9,12$ & $\mathrm{~g}, \mathrm{~m}$ & - & & 2 & 2 & 0 & 2 & 0 \\
\hline $\mathrm{O}: 11(\mathrm{~F})$ & Rubislaw & I & 11 & $\mathrm{r}$ & $e, n, x$ & & 1 & 1 & 0 & 1 & 0 \\
\hline \multirow[t]{3}{*}{$\mathrm{O}: 13(\mathrm{G})$} & Cubana & $\mathrm{I}$ & $\underline{1}, 13,23$ & $\mathrm{z}_{29}$ & - & {$\left[\mathrm{z}_{37}\right],\left[\mathrm{z}_{43}\right]$} & 2 & 2 & 0 & 2 & 0 \\
\hline & Putten & I & 13,23 & $\mathrm{~d}$ & $1, \mathrm{w}$ & & 1 & 1 & 0 & 1 & 0 \\
\hline & Worthington & $\mathrm{I}$ & $\underline{1}, 13,23$ & $\mathrm{z}$ & $1, \mathrm{w}$ & {$\left[\mathrm{Z}_{43}\right]$} & 1 & 1 & 0 & 1 & 0 \\
\hline $\mathrm{O}: 35(\mathrm{O})$ & Widemarsh & I & 35 & $\mathrm{z}_{29}$ & - & & 1 & 1 & 0 & 1 & 0 \\
\hline \multirow[t]{2}{*}{ O:40 ( R) } & Johannesburg & $\mathrm{I}$ & $\underline{1,40}$ & $\mathrm{~b}$ & $\mathrm{e}, \mathrm{n}, \mathrm{x}$ & & 1 & 1 & 0 & 1 & 0 \\
\hline & & & & & & Total $^{\mathrm{d}}$ & 111 & 98 & 10 & 96 & 12 \\
\hline
\end{tabular}

Abbreviations: No., number; SeqSero, sequence serotyping tool; SISTR, Salmonella in silico typing resource tool; TP, true positive; FP, false positive.

${ }^{a}$ With the exception of serotype I:4,5,12:i:-, neither tool was able to predict the serotype of isolates designated by a formula (these isolates were missing one or more antigens according to in-lab serotyping). Instead both tools predicted a serotype closely related to the antigenic formula. Formulas are listed below their closest matching serotype.

${ }^{\mathrm{b}}$ Underlined $\mathrm{O}$ factors are phage-determined epitopes. Rough variants are isolates that do not express an $\mathrm{O}$ antigen.

${ }^{\mathrm{c}}$ Some isolates express abnormal $\mathrm{R}$ phases and/or a third $\mathrm{H}$ antigen. $\mathrm{R}$ phases agglutinable by anti-1,2 $-1,5-1,6-1,7$ sera but not by anti-2 $-5-6-7$ sera are designated by R1.

${ }^{\mathrm{d}}$ Inconclusive and incongruent results are not included in totals for TP and FP

*Numbers reported are for analysis of assembled sequences with SISTR and raw-read sequences with SeqSero. Number with adjacent -I flag indicates an inconclusive result where $>1$ serotypes were provided by tool. False positive results were incongruent results for closely related serotype with a similar antigenic formula.

[] = antigenic factors that may be present or absent

$0=$ antigenic factor(s) that are weakly agglutinable

\{\}$=$ exclusive $\mathrm{O}$-factors (cannot exist with other factors in curly brackets) 
Table 2.6. Accuracy of AMR phenotype predictions in Salmonella by AMR-gene prediction tools

\begin{tabular}{|c|c|c|c|c|c|c|c|}
\hline \multirow[b]{3}{*}{ Antibiotic } & \multirow[b]{3}{*}{$\mathrm{ECV}\left(\mu \mathrm{g} \mathrm{mL}^{-1}\right)$} & \multirow{3}{*}{$\begin{array}{l}\text { AMR Prediction } \\
\text { Tool* }\end{array}$} & \multicolumn{4}{|c|}{ No. of test results } & \multirow{3}{*}{$\begin{array}{c}\text { Accuracy } \\
(\%)\end{array}$} \\
\hline & & & \multicolumn{2}{|c|}{ Phenotype: Sensitive (S) } & \multicolumn{2}{|c|}{ Phenotype: Resistant (R) } & \\
\hline & & & Genotype:R & Genotype:S & Genotype:R & Genotype:S & \\
\hline \multicolumn{8}{|l|}{ Aminoglycosides } \\
\hline GEN & $\mathrm{S}: \leq 4 \quad \mathrm{R}:>16$ & All & 0 & 100 & 10 & 1 & 99.1 \\
\hline KAN & $\mathrm{S}: \leq 16 \quad \mathrm{R}: \geq 64$ & All & 0 & 110 & 1 & 0 & 100.0 \\
\hline STR & $\mathrm{S}: \leq 32 \quad \mathrm{R}: \geq 64$ & All & 13 & 72 & 24 & 2 & 86.5 \\
\hline $\mathrm{STR}^{\mathrm{a}}$ & $\mathrm{S}: \leq 16 \quad \mathrm{R}: \geq 32$ & All & 4 & 72 & 33 & 2 & 94.6 \\
\hline \multicolumn{8}{|l|}{ Beta-lactams } \\
\hline $\begin{array}{l}\text { AMC } \\
\text { Cenhalosnorins }\end{array}$ & $\mathrm{S}: \leq 8 \quad \mathrm{R}: \geq 32$ & All & 0 & 91 & 20 & 0 & 100.0 \\
\hline FOX & $\mathrm{S}: \leq 8 \quad \mathrm{R}: \geq 32$ & All & 0 & 91 & 20 & 0 & 1000 \\
\hline TIO & $\mathrm{S}: \leq 2 \quad \mathrm{R}: \geq 8$ & All & 0 & 91 & 20 & 0 & 100.0 \\
\hline $\mathrm{CRO}$ & $\mathrm{S}: \leq 16 \quad \mathrm{R}: \geq 4$ & All & 0 & 91 & 20 & 0 & 100.0 \\
\hline \multicolumn{8}{|l|}{ Penicillin } \\
\hline AMP & $\mathrm{S}: \leq 8 \quad \mathrm{R}: \geq 32$ & All & 0 & 84 & 27 & 0 & 100.0 \\
\hline $\begin{array}{l}\text { Phenicol } \\
\text { CHL } \\
\text { Folate Pathway In }\end{array}$ & $\mathrm{S}: \leq 8 \quad \mathrm{R}: \geq 32$ & All & 0 & 105 & 6 & 0 & 100.0 \\
\hline & & & 0 & 86 & 25 & 0 & 100.0 \\
\hline SOX & $\mathrm{S}: \leq 256 \quad \mathrm{R}: \geq 512$ & ResFinder v2.1* & 0 & 86 & 24 & 1 & 99.1 \\
\hline SXT & $\mathrm{S} \cdot<2 \quad \mathrm{R} \cdot>4$ & & 0 & 103 & 8 & 0 & 100.0 \\
\hline \multirow{2}{*}{\multicolumn{8}{|c|}{ Tetracycline }} \\
\hline & & & & & & & \\
\hline TCY & $\mathrm{S}: \leq 4 \quad \mathrm{R}: \geq 16$ & All & 0 & 80 & 31 & 0 & 100.0 \\
\hline
\end{tabular}

*Except where ResFinder v2.1 and KMA listed separately, all AMR prediction tools (CARD-RGI, srst2 -ResFinder -ARGannot -NCBI, KMA -assemblies and -raw-reads

(ResFinder database), and ResFinder-v2.1 and -v3.1 server through centre for genomic epidemiology website) performed equally.

${ }^{a}$ Streptomycin testing for $\mathrm{ECV} \leq 16\left(\mu \mathrm{g} \mathrm{mL}^{-1}\right)$ was conducted using the agar dilution method. All other resistance phenotypes were determined by broth microdilution using the Sensititre system (Trek Diagnostic Systems, Cleveland, OH, USA).

ECVs according to CLSI guidelines.

Abbreviations:ECV, epidemiological cutoff value; AMR, antimicrobial resistance; ResFinder, Resistance Finder; CARD-RGI, Comprehensive Antibiotic Resistance Database -

Resistance Gene Identifier; SRST2, short read sequence typing v0.2.0; NCBI, National Center for Biotechnology Information; KMA, $k$-mer alignment; GEN, gentamicin; KAN,

kanamycin; STR, streptomycin; AMC, amoxicillin-clavulanic acid; FOX, cefoxitin; TIO, ceftiofur; CRO, ceftriaxone; AMP, ampicillin; CHL, chloramphenicol; SOX,

sulfisoxazole; SXT, trimethoprim-sulfamethoxazole; TCY, tetracycline . 
With some exceptions, resistance to antimicrobials in the 58 resistant Salmonella strains (including 223 AMR phenotypes) was accurately predicted (>99\%) based on genotype (Table 2.6). There were originally seventeen discrepancies where an ARG was detected yet the isolate was phenotypically sensitive. Repeat testing of isolates OLC2589, OLC2594, OLC2622, and OLC2644 by broth microdilution confirmed WGS-based predictions of FOX; GEN and TCY; FOX; FOX and AMX resistance, respectively (Table 2.6, Table 2.7, Table S2.2). The remaining thirteen isolates were predicted to be STR-resistant but were deemed sensitive based on an epidemiological cutoff value (ECV) of $\geq 64 \mu \mathrm{g} / \mathrm{mL}$. Due to discrepancies in STR phenotypic and genomic resistance, Tyson et al. (2016) suggested that STR epidemiological cutoff values be lowered to resistance at $\geq 32 \mu \mathrm{g} / \mathrm{mL}$. All isolates were re-tested for STR-resistance by agar dilution and the reduced ECV $\geq 32 \mu \mathrm{g} / \mathrm{mL}$ was applied. This decreased the number of false positive STRresistant genotypes from thirteen to four (Table 2.6, Table S2.2). Three false negative genotypes in which there were no detected ARGs by any of the tools used in this study yet phenotypic resistance to GEN (OLC2626) or STR (OLC2536 and OLC2644) were observed. Broth microdilution testing of these isolates confirmed original phenotypic testing (Table 2.6, Table 2.7, Table S2.2).

Following verification of discrepancies with repeat testing, the accuracy of predicting AMR based on ARG detection was determined (Table 2.6). The accuracy for all tools was $>99 \%$ for predicting resistance to aminoglycosides GEN and KAN; $\beta$ lactams AMC, FOX, TIO, CRO, and AMP; the phenicol CHL; TCY; and the folate synthesis inhibitor SOX (Table 2.6). The accuracy of predicting phenotypic AMR to SXT was $100 \%$ for all tools except for KMA-analysis of assembled genomes (Table 2.6). The 
accuracy for genotypic prediction of phenotypic STR resistance increased from $86.5 \%$ to 94.6\% when the ECV was lowered from $64 \mu \mathrm{g} / \mathrm{mL}$ to $32 \mu \mathrm{g} / \mathrm{mL}$.

To determine if ARGs were plasmid- or chromosomally-encoded, samples were analysed with MOB-suite v. 1.4.1 (Robertson and Nash, 2018). Many of the genes were predicted to be plasmid encoded, while some genes were exclusively determined to occur within the chromosomal sequences (aac(6’)-Iaa, fosA7, Table 2.3, Table S2.3). In some cases, ARGs were predicted by MOB-suite in both locations $(s u l 1$, floR) (Table 2.3, Table S2.3) (Robertson and Nash, 2018).

\subsubsection{Minimum Coverage Requirements for Accurate ARG Determination}

To determine sequence coverage requirements for accurate ARG detection, a simulated dataset was analysed with KMA. Sequence data for each of the 111 Salmonella isolates were subsampled to generate sequence coverages ranging from $1 \mathrm{X}$ to $20 \mathrm{X}$, with 100 replicates at each coverage level (Figure 2.2 .1 ). With a $98 \%$ target-gene identity cutoff and 20X genome coverage, the percent of ARGs correctly identified was $>90 \%$ for all genes except $\operatorname{ant}\left(3^{\prime \prime}\right)$-Ia, and aadA3 which were detected $73.7 \%$ and $70.7 \%$ respectively. At $20 \mathrm{X}$ genome coverage with a $90 \%$ target-gene identity, ARG detection was $>98 \%$ for

all genes except ant(3'))-Ia, aadA3, and $d f r A 15 b$ which were detected in 94.9, 97.7, and $97.7 \%$ of the simulated datasets respectively (Figure 2.1 ). At $80 \%$ and $90 \%$ identity ARGs were accurately identified; however, occasionally alternative alleles were reported for genes $\operatorname{aad} A$, tetA, and $d f r A 14$ (data not shown). 
Table 2.7. Broth microdilution testing of isolates with genotype-phenotype discrepancies

\begin{tabular}{|c|c|c|c|c|c|c|c|c|c|c|c|c|c|}
\hline Isolate & & Neg. & $\begin{array}{l}\text { S. ser. } \\
\text { Anatum } \\
\text { var.15+ }\end{array}$ & $\begin{array}{l}\text { S. ser. } \\
\text { Hadar }\end{array}$ & $\begin{array}{l}\text { S. ser. } \\
\text { Heidelberg }\end{array}$ & $\begin{array}{l}\text { S. ser. } \\
\text { Heidelberg }\end{array}$ & S. ser. Infantis & $\begin{array}{l}\text { S. ser. } \\
\text { Kentucky }\end{array}$ & $\begin{array}{l}\text { S. ser. } \\
\text { Kentucky }\end{array}$ & $\begin{array}{l}\text { S. ser. } \\
\text { Kiambu }\end{array}$ & $\begin{array}{l}\text { S. ser. } \\
\text { Muenchen }\end{array}$ & $\begin{array}{l}\text { S. ser. } \\
\text { Saintpaul }\end{array}$ & E coli \\
\hline Antibiotic & Analysis* & & OLC2536 & OLC2537 & OLC2644 & OLC2564 & OLC2545 & OLC2589 & OLC2622 & OLC2576 & OLC2626 & OLC2594 & ATCC25922 \\
\hline \multirow{4}{*}{ TCY } & Sensititre & $\mathrm{N} / \mathrm{A}$ & $<=4$ & $>32$ & $<=4$ & $>32$ & $>32$ & $<=4$ & $>32$ & $<=4$ & $<=4$ & $<=4$ & N/A \\
\hline & Genotype & N/A & - & tet $A$ & - & tet $A$-like & tet $A$-like & - & tet $B$ & - & - & tet $A$ & - \\
\hline & BMD1 & 0 & 1 & $>64$ & 1 & $>64$ & 32 & 1 & $>64$ & 1 & 1 & $>64$ & 1 \\
\hline & BMD2 & 0 & 4 & $>64$ & 4 & $>64$ & $>64$ & 1 & $>64$ & 4 & 4 & $>64$ & 1 \\
\hline \multirow{4}{*}{ GEN } & Sensititre & N/A & 0.5 & 0.5 & $<=0.25$ & $>16$ & $>16$ & $<=0.25$ & 0.5 & $>16$ & $>16$ & 4 & N/A \\
\hline & Genotype & N/A & - & - & - & aac(3)-VIa & $a a c(3)-V I a$ & - & - & $a a c(3)-V I a$ & - & $a a c(3)-V I a$ & - \\
\hline & BMD1 & 0 & 8 & 4 & 2 & $>16$ & $>16$ & 4 & 4 & $>16$ & $>16$ & $>16$ & 2 \\
\hline & BMD2 & 0 & 2 & 2 & 4 & $>16$ & $>16$ & 2 & 2 & $>16$ & $>16$ & $>16$ & 1 \\
\hline \multirow{4}{*}{ CEF } & Sensititre & N/A & 4 & 2 & 16 & 2 & $>32$ & 16 & 16 & 4 & 4 & $>32$ & N/A \\
\hline & Genotype & N/A & - & - & blaCMY-2 & - & blaCMY-2 & blaCMY-2 & blaCMY-2 & - & - & blaCMY-2 & - \\
\hline & BMD1 & 0 & 4 & 2 & $>32$ & 2 & $>32$ & 32 & 32 & 4 & 4 & $>32$ & 2 \\
\hline & BMD2 & 0 & 4 & 2 & $>32$ & N/A & $>32$ & $>32$ & $>32$ & 4 & 4 & $>32$ & 2 \\
\hline \multirow{4}{*}{ AMC } & Sensititre & $\mathrm{N} / \mathrm{A}$ & $<=1$ & $<=1$ & 16 & $<=1$ & $>32$ & 32 & $>32$ & $<=1$ & $<=1$ & $>32$ & $\mathrm{~N} / \mathrm{A}$ \\
\hline & Genotype & N/A & - & - & blaCMY-2 & - & blaCMY-2 & blaCMY-2 & blaCMY-2 & - & - & blaCMY-2 & - \\
\hline & BMD1 & 0 & 2 & 2 & $>64$ & 2 & $>64$ & $>64$ & $>64$ & 4 & 4 & $>64$ & 32 \\
\hline & BMD2 & 0 & 4 & 4 & $>64$ & 4 & $>64$ & $>64$ & $>64$ & 4 & 4 & $>64$ & 32 \\
\hline \multirow{5}{*}{ STR } & Sensititre & N/A & 64 & $>64$ & $<=32$ & 64 & $>64$ & $<=32$ & 64 & $>64$ & $<=32$ & $<=32$ & N/A \\
\hline & Genotype & $\mathrm{N} / \mathrm{A}$ & - & $\operatorname{str} A, \operatorname{str} B$ & - & $\operatorname{aadAl}$ & aadA1,strA,strB & - & $\operatorname{str} A, s t r B$ & aadAl & - & aadAl & - \\
\hline & Agar & N/A & 32 & 64 & 32 & 64 & 64 & 4 & 64 & 64 & $<=4$ & 32 & $<=4$ \\
\hline & BMD1 & 0 & $>128$ & $>128$ & 32 & $>128$ & $>128$ & 16 & $>128$ & $>128$ & 16 & $>128$ & 8 \\
\hline & BMD2 & 0 & $>128$ & $>128$ & 32 & $>128$ & $>128$ & 16 & $>128$ & $>128$ & 8 & $>128$ & 8 \\
\hline
\end{tabular}

Abbreviations: Neg, negative control; S., Salmonella enterica; ser, serotype; TCY, tetracycline; GEN, gentamicin; CEF, cefoxitin; AMC, amoxicillin/clavulanic acid; STR, streptomycin; BMD1 and BMD2, broth microdilution replicates 1 and 2; N/A, not applicable (not conducted for this isolate in this study).

*Analysis of phenotype includes original Sensititre analysis, broth microdilution replicates 1 and 2 (BMD1 and BMD2, respectively), and the agar dilution method conducted for streptomycin (Agar).

Grey cells indicate discrepancy between genotype prediction and phenotype.

Escherichia coli ATCC25922 was included as a sensitive control 
Figure 2.1. Genome coverage required to detect ARGs. (Page 58) Various levels of sequence coverage (1X, 2.5X, 5X, 10X, 15X, 20X) were subsampled 100 times from rawreads of sequence files for each of 111 Salmonella isolates. (A) Aminoglycoside resistance genes, (B) Beta-lactamase resistance genes, (C) Phenicol, florfenicol, and tetracycline resistance genes, (D) Trimethoprim and sulphonamide resistance genes. Each of the subsampled sequences was analysed for ARGs using KMA v 1.17 and the ResFinder database. Percent correctly identified at $80 \%$ (continuous line), 90\% (dashed line) and $98 \%$ (dotted line) gene identity was determined by dividing the total number of hits by the expected number of hits. The X-axis represents the sampled fold genome coverage. Genes are differentiated by colour and shape (Gene). 

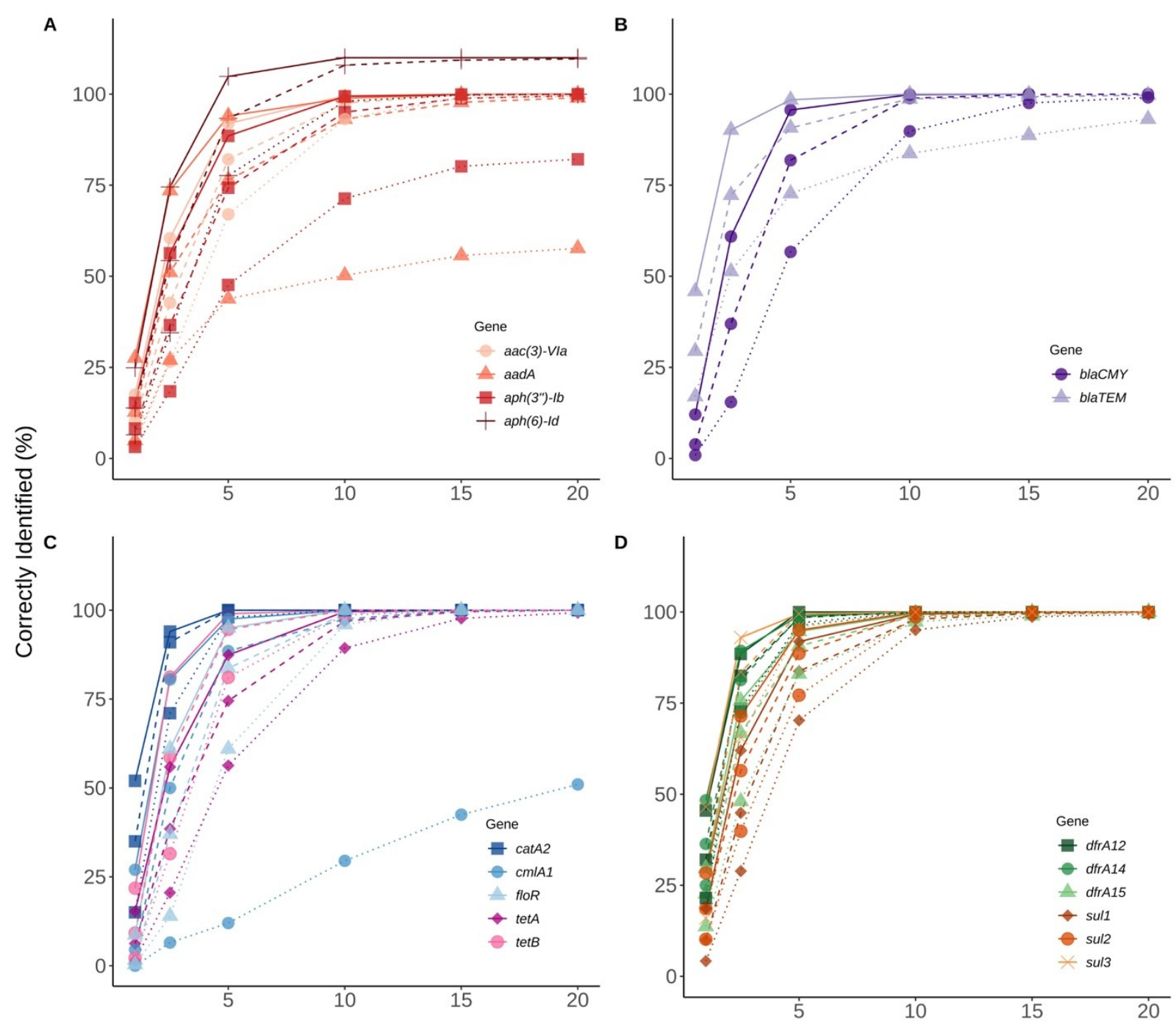

D

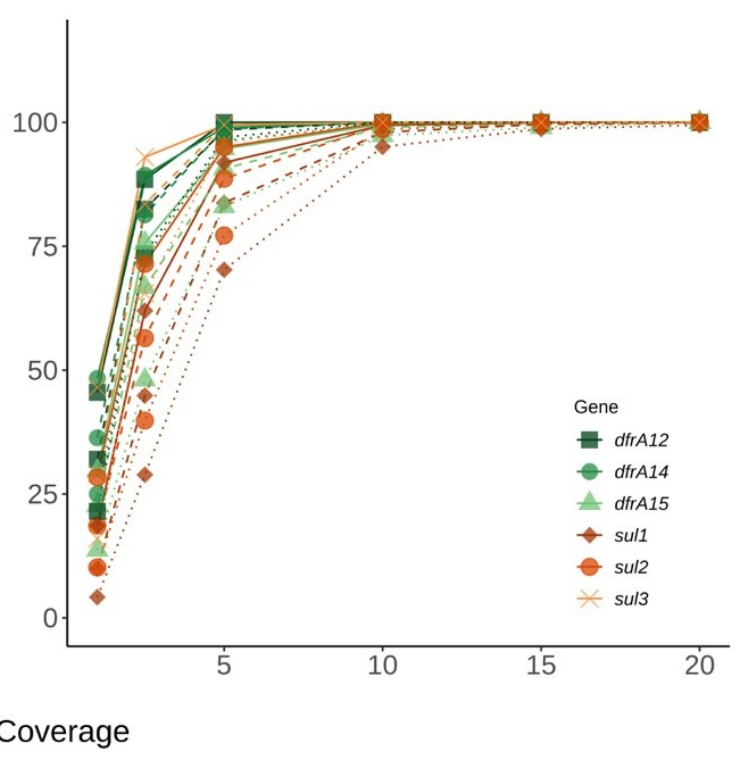

Gene Identity Cutoff $(\%)-80 \quad-\quad 90 \quad \cdots \quad 98$ 


\subsubsection{Effects of Assembly on ARG Detection}

To determine impact of genome assembly on ARG detection, raw-reads were subsampled from the WGS data of seven isolates at $5 \mathrm{X}, 10 \mathrm{X}, 15 \mathrm{X}$ and $20 \mathrm{X}$ genome coverages then assembled with both SKESA and SPAdes. Isolates were selected to include various ARG profiles, including a sensitive isolate (Table 2.3). ARG detection in sub-sampled SKESA and SPAdes assemblies was compared to detection in sub-sampled raw reads at each coverage level using the KMA tool (Table 2.1, Figure 2.2). Significant differences were observed at $5 \mathrm{X}$ genome coverage between SKESA assemblies and both SPAdes assemblies and raw-read sequences for all ARGs except $d f r A 14$ (Figure 2.2). As coverage increased AMR predictions with either assembly method and raw-reads improved. Compared to SKESA, blaCMY-2 was more reliably detected in SPAdesassembled and raw-read sequence data at $5 \mathrm{X}, 10 \mathrm{X}$, and $15 \mathrm{X}$ genome coverage (Figure 2.2). Similarly, aac(3)-Vla, floR, sull, sul2, and tetA were detected at significantly higher proportions in SPAdes assemblies and raw-reads compared to SKESA assemblies at 10X coverage. Overall, strA had a lower detection frequency than the other ARGs in assembled genomes. This gene was only detected twice out of 20 replicate assemblies in one isolate (OLC2568). Further investigation of the genome found the $\operatorname{str} A$ gene among two smaller separated fragments in the assembled genomes. Annotation of the OLC2568 sequence revealed the insertion of the dihydrofolate reductase gene $d$ frA 14 in the middle of the strA coding region. Detection of strA was significantly higher at all coverage levels using raw-read sequence data, and SPAdes outperformed SKESA at coverage levels of 5X and 10X. Table 2.3 depicts the location of the ARGs in the seven test isolates. 
Figure 2.2. Effects of sequence coverage and assembly on ARG detection. (Page 61) Levels of $5 \mathrm{X}, 10 \mathrm{X}, 15 \mathrm{X}$ and $20 \mathrm{X}$ genome coverage were subsampled 20 times from rawreads of sequence files for seven Salmonella isolates and assembled using both SPAdes and SKESA. Panels are separated by gene (listed at top of each panel). Proportion gene was identified out of $n$ trials $(n=20,40,60$, or 80 depending on gene) is plotted on $y-$ axis with upper and lower $95 \%$ confidence intervals. Significance of proportion detected between assemblies and raw-reads was determined for each gene at each coverage level using Fisher's exact test. Significance values are displayed above corresponding data points: $\mathrm{p}<0.05=* ; \mathrm{p}<0.01=* * ; \mathrm{p}<0.001=* * * ; \mathrm{p}<0.0001=* * * *$ 


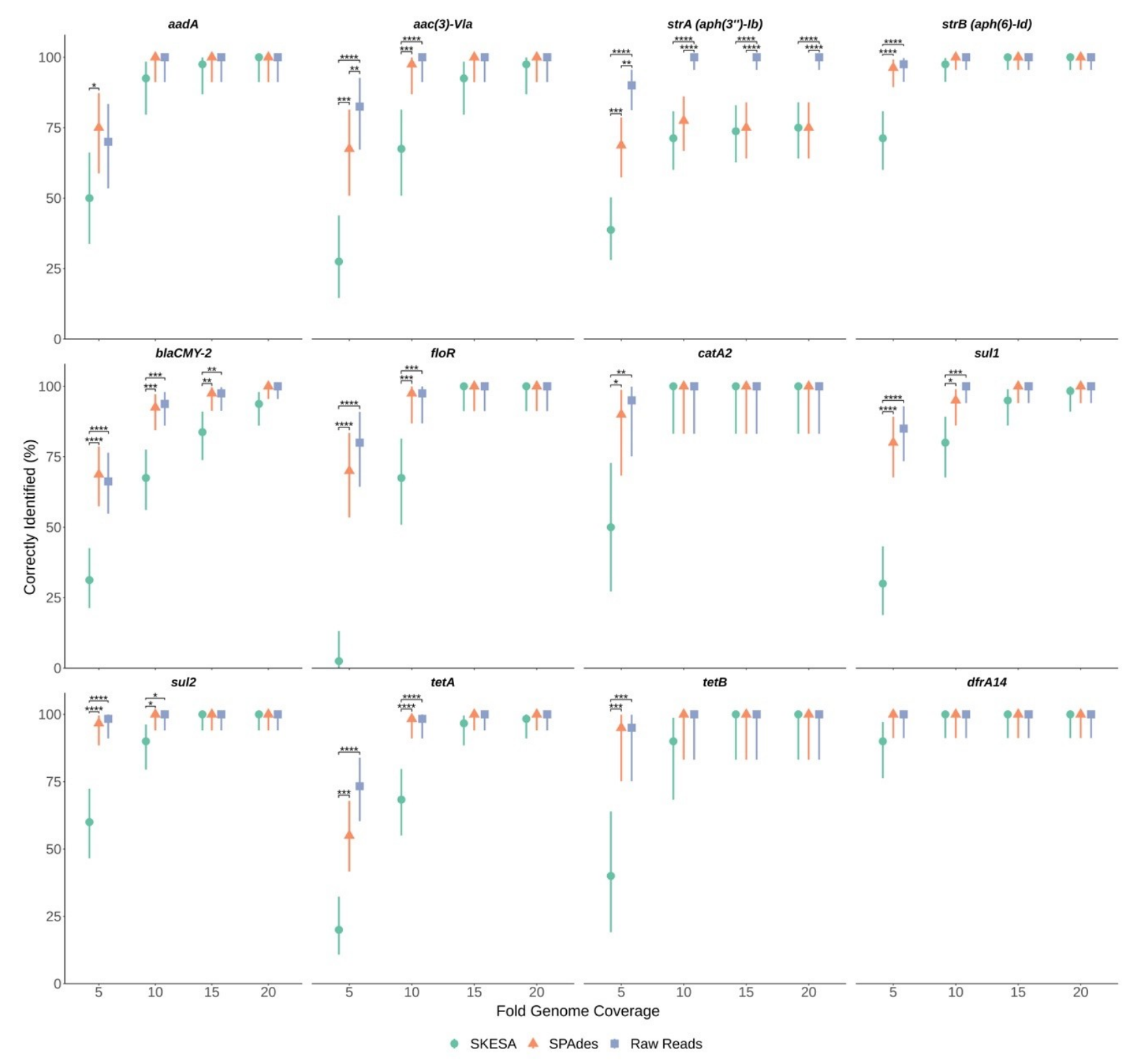




\subsubsection{Single Nucleotide Polymorphisms (SNPs) Conferring AMR}

CGE's PointFinder program was used to investigate SNVs known to confer resistance to antibiotics (Zankari et al., 2012). Two isolates (OLC2588 and OLC2622) with phenotypic NAL resistance and intermediate ciprofloxacin resistance had SNVs in gyrA resulting in non-synonymous mutations at amino acid 83 (Table 2.2, Table S2.2); mutations at this position are known to confer quinolone resistance (Ruiz et al., 1997; Piddock et al., 1998; Hakanen et al., 1999; Reche et al., 2002; Cooper et al., 2015, 2016).

To identify the genetic basis of STR and GEN resistance in the three isolates (OLC2536, OLC2644, and OLC2626) with no identified ARGs, SNV-based analyses were conducted on all isolates in this study to identify mutations in genes that have been associated with increased aminoglycoside resistance. No non-synonymous mutations or truncations were found in genes $g \ln A, u b i E$ and $r p s L$ in any of the 111 isolates. Multiple non-synonymous mutations were found in $\operatorname{gid} B, \operatorname{cyoB}, \operatorname{cyoC}$, $\operatorname{trk} H$, isp $A, n u o E, u b i F$, and $\operatorname{aroD}$ (data not shown), and one non-synonymous mutation was found for $f u s A$, yet no associated phenotypic resistance associated with these mutations were observed. A small number of non-synonymous mutations were also observed for $n u o F, p r f B$, and $u b i A$ in a few isolates, some of which could possibly alter function (Table 2.2).

Comparison of the Nuo protein complex of STR resistant OLC2536 to S. enterica ser. Anatum var. $15+$ genomes in the OLC-CFIA collection as well as a publicly available STR sensitive Salmonella ser. Anatum var. 15+ genome (Accession: NZ_CP013222) revealed a SNV in the nuoF coding region. This resulted in a nonsynonymous 45-CTG (Leu) $\rightarrow$ CGG (Arg) mutation. Further analysis of the aligned nuoF region using the NCBI blast database found the 45-CTG codon to be highly 
conserved in the $n u o F$ region of aligned Salmonella genomes (all results exhibited 99\% identity to OLC2536 in this study). This gene was highly conserved among all isolates tested with only four of 111 isolates exhibiting $<100 \%$ amino acid identity to the sensitive reference. Out of the four isolates only OLC2536 exhibited a L45R substitution while three other isolates had P378S ( $\mathrm{n}=1$ ) or K257R ( $\mathrm{n}=2)$ substitutions (Table 2.2). Of the isolates with non-synonymous mutations in $n u o F$, only OLC2536 presented with phenotypic STR resistance. Two other S. enterica ser. Anatum isolates (one confirmed var. 15+) in the CFIA collection also harbored the L45R mutation with no other ARGs and were phenotypically STR-resistant (data not shown). Two more distantly related $S$. enterica ser. Anatum isolates (not var. 15+, approximately $160 \mathrm{SNV}$ difference with OLC2536) which encoded leucine at codon 45 of nuoF and did not harbor any STRresistance genes were phenotypically STR-sensitive.

Comparison of Salmonella ser. Heidelberg isolates OLC2644 (STR resistant) and OLC2552 (STR sensitive) found ten SNVs. SNVs were located in the DUF3626 domaincontaining protein (accession: WP_000917268.1), the phosphoenolpyruvate-dependent sugar phosphotransferase system (PTS) galactitol-specific EIIC component gatC (accession: WP_000460837.1), L-cystine-binding protein fliY (accession: WP_000949370.1), tetR/acrR family transcriptional regulator (accession: WP_000208474.1), sensor kinase $d p i B$ (accession: ACF66636.1), yjj I family glycine radical enzyme (accession: WP_001111688.1), nickel/cobalt transporter (accession: WP_000111019.1), a hypothetical protein (accession: WP_107321080.1), and an uncharacterized genomic region. Nonsynonymous mutations were located in citA 
(Q324L), yjjI (L499Q), and a nickel/cobalt transporter (G51D) of OLC2644. A nonsense mutation also occurred in fliY of OLC2644 at codon 202.

Comparison of Salmonella ser. Muenchen isolates OLC2626 (GEN resistant) and OLC2592 (GEN sensitive) resulted in three SNVs. SNVs were located in the bifunctional glycosyltransferase/transpeptidase penicillin binding protein 1 gene $\operatorname{mrc} B$ (accession: WP_052934909.1), phosphate inducible starvation protein gene $p s i E$ (accession: WP_000982749.1), and a DUF1176 domain-containing protein of unknown function (accession: WP_001270678.1). None of these SNPs resulted in nonsynonymous mutations. OLC2592 also harbored a plasmid encoding resistance to sulphonamides (sul2), tetracycline (tetA), and streptomycin (strA and $\operatorname{str} B)$.

\subsubsection{Minimal media induces cryptic aadA in Salmonella spp. isolates}

To study the impact of minimal media (M9) on STR resistance, a subset including both STR sensitive and resistant isolates was tested by broth and agar microdilution (Table 2.8). Differences were observed for STR MICs in broth compared to agar for all isolates tested. All isolates grew on all agar formulations without STR, except OLC2542 which exhibited limited growth on M9. Increased STR MICs were observed both in M9 and LB agar and broth compared to MH for most isolates, even those characterized as STR sensitive (Table 2.8). With the exception of OLC2542 and E. coli ATCC 25922 (sensitive control), all isolates exhibited extremely high MICs ( $\geq 64 \mu \mathrm{g} / \mathrm{ml})$ in M9 media (Table 2.8). 
Table 2.8. STR minimum inhibitory concentration of Salmonella isolates in MH, LB, and M9 agar and broth

\begin{tabular}{|c|c|c|c|c|c|c|c|c|}
\hline \multirow{3}{*}{ Isolate } & \multirow{3}{*}{ Serotype } & \multirow{3}{*}{$\begin{array}{l}\text { Aminoglycoside } \\
\text { Resistance } \\
\text { Gene(s) }\end{array}$} & \multicolumn{6}{|c|}{ MIC Streptomycin $(\mu \mathrm{g} / \mathrm{ml})^{\mathrm{a}}$} \\
\hline & & & \multicolumn{3}{|c|}{ Agar } & \multicolumn{3}{|c|}{ Broth } \\
\hline & & & MH & LB & M9 & MH & LB & M9 \\
\hline OLC2536 & Anatum var. $15+$ & & 64 & $>128$ & $>128$ & $>64$ & $>64$ & $>64$ \\
\hline OLC2540 & Heidelberg & $\operatorname{aph}\left(3^{\prime}\right)-\mathrm{Ia}$ & 8 & 32 & $>128$ & 16 & 64 & $>64$ \\
\hline OLC2541 & Heidelberg & $\begin{array}{l}\text { aadAl-like, strA, } \\
\text { strB }\end{array}$ & $>128$ & $>128$ & $>128$ & $>64$ & $>64$ & $>64$ \\
\hline OLC2542 & Heidelberg & $\begin{array}{l}\text { aac(3)-VIa-like, } \\
\text { aadA1 }\end{array}$ & 64 & $>128$ & $\mathrm{LG}$ & $>64$ & $>64$ & LG \\
\hline OLC2548 & Ohio & $\operatorname{aadAl}$ & 32 & 128 & $>128$ & $>64$ & $>64$ & $>64$ \\
\hline OLC2560 & Ohio & & 4 & 8 & 128 & 16 & 16 & $>64$ \\
\hline OLC2561 & Ohio & & 8 & 16 & 128 & 16 & 32 & $>64$ \\
\hline OLC2568 & Heidelberg & $\begin{array}{l}\text { str } A \text {-like, } \\
\text { str } B \text {-like }\end{array}$ & 8 & 32 & $>128$ & 16 & 64 & $>64$ \\
\hline OLC2575 & Kiambu & str $A$-like, str $B$ & 8 & 32 & $>128$ & 16 & 64 & $>64$ \\
\hline OLC2576 & Kiambu & $\begin{array}{l}\text { aac(3)-VIa-like, } \\
\text { aadA1 }\end{array}$ & 64 & $>128$ & $>128$ & $>64$ & $>64$ & $>64$ \\
\hline OLC2577 & Kiambu & & 8 & 32 & $>128$ & 32 & 32 & $>64$ \\
\hline OLC2592 & Muenchen & str $A, \operatorname{str} B$-like & 32 & 128 & $>128$ & $>64$ & $>64$ & $>64$ \\
\hline OLC2596 & Thompson & aadA1, aadA2 & 16 & 64 & $>128$ & 32 & $>64$ & $>64$ \\
\hline OLC2597 & Thompson & $\operatorname{aad} A 1, \operatorname{aad} A 2$ & 16 & 64 & $>128$ & $>64$ & $>64$ & $>64$ \\
\hline OLC2626 & Muenchen & & 4 & 8 & 64 & 8 & 16 & $>64$ \\
\hline OLC2634 & Thompson & & 8 & 16 & $>128$ & NT & NT & NT \\
\hline ATCC25922 & E. coli & negative control & 4 & 8 & 4 & 8 & 16 & 8 \\
\hline
\end{tabular}

Abbreviations: STR, streptomycin; AMR, antimicrobial resistance; MIC, minimum inhibitory concentration; MH, Mueller Hinton; LB, Luria-Bertani Lennox formulation; M9, minimal salts media; NT, not tested; LG, limited growth (no growth after 24 hours).

${ }^{\mathrm{a}} \mathrm{MIC}$ of $\geq 32 \mu \mathrm{g} / \mathrm{ml}$ in $\mathrm{MH}$ is considered resistant. 


\subsection{Discussion}

Salmonella colonize a range of animal hosts; consequently, in industrialized countries, the majority of human infections are associated with contaminated animal food products (Butaye et al., 2006). Specific Serotypes and AMR profiles can be linked to food commodities which can vary depending on antimicrobial usage for food production in different countries (Butaye et al., 2006; Zhao et al., 2007; Hong et al., 2016; Yoon et al., 2017). As such, the resistance profile and serotype of a Salmonella isolate can provide clues as to the epidemiology of an infection. Some examples include MDR $S$. ser. Newport associated with exposure to dairy cattle and beef in the US (Holmberg et al., 1984; Centers for Disease Control and Prevention (CDC), 2002; Butaye et al., 2006; Schneider et al., 2011; Plumb, 2019), MDR S. ser. Heidelberg frequently associated with poultry in both Canada and the US (Dutil et al., 2010; Routh et al., 2015; Gieraltowski et al., 2016), and MDR $S$. ser. Paratyphi B variant Java has been linked to poultry in Europe (van Pelt et al., 2003; Threlfall et al., 2005; Butaye et al., 2006). The association between AMR phenotype and serotype could provide valuable clues as to the possible source of infection for risk assessment and epidemiological investigations.

Genome-based prediction of Salmonella serotype and AMR is increasingly being used by public-health organizations worldwide (Inouye et al., 2014; Bradley et al., 2015; Licker et al., 2015; Tyson et al., 2015, 2016; Clausen et al., 2016; McDermott et al., 2016; Zhao et al., 2016). These predictions are conducted using a variety of computational algorithms which rely on different databases, with few comparative analyses of approaches. We found that AMR and serotype could be accurately predicted in Salmonella from WGS data using several widely available programs with minimal 
differences. While approaches for genoserotyping rely on similar antigen markers (Yachison et al., 2017; Uelze et al., 2020), for AMR there are currently multiple databases containing lists of known resistance genes/mutations, with most databases focusing on acquired ARGs with implications in human and veterinary medicine (Inouye et al., 2014; Bradley et al., 2015; Licker et al., 2015; Tyson et al., 2015, 2016; Clausen et al., 2016; McDermott et al., 2016; Zhao et al., 2016; Feldgarden et al., 2019). The phenotype prediction tools tested in this study provided similar results with minimal variation. Variability observed in this study can be explained by differences among databases, application of computational algorithms, or difficulties in detection of AMR resulting from point mutations. Overall WGS analysis was more reliable than phenotyping as it identified several discordant results that were corrected upon retesting.

\subsubsection{Reliability of Salmonella serotyping tools}

SeqSero and SISTR determine Salmonella serotypes from WGS data based on matches to genes encoding somatic and flagellar antigens (Zhang et al., 2015; Zhang et al., 2019; Yoshida et al., 2016). The in silico tools then use the predicted antigenic formula to determine the most likely named serotype in the Kauffmann-White-Le Minor scheme (Grimont and Weill, 2007) (Table 2.5, Table S2.1). Both of these tools performed well for serotype determination for the small set of isolates included in this study, with unambiguous identification of serovars for over 88.3 and $86.5 \%$ of isolates using SISTR and SeqSero2, respectively, and no "incorrect" serotype identification (Table 2.4). "Incongruent" results were observed for ten of the isolates included in this study where genes encoding antigens were detected in WGS data, but were not expressed based on 
serotyping results. Yachison et al. (2017) suggest a need to carry out further analyses using traditional serotyping for incongruent results and to "reframe serotyping for genomics", as genes that are carried by an isolate are not necessarily expressed. As in previous studies, SISTR performed slightly better than SeqSero2 for resolving "inconclusive" results due to use of cgMLST for distinguishing serovars with the same antigenic profile (Table 2.4, Table S2.1) (Uelze et al., 2020; Yachison et al., 2017). However, both tools generated "inconclusive" results for the three Salmonella ser. Othmarshen isolates included in this study. This difficulty distinguishing S. ser Othmarshen and Oranienberg has been described by Robertson et al. (2018). The authors provide evidence that these two serovars are not genetically distinct and therefore not easily resolved using in silico serotyping tools.

The performance of tools for in silico Salmonella serotyping has been extensively evaluated elsewhere. For example, Yachison et al. (2017) and Uelze et al. (2020) evaluated SISTR, SeqSero version 1 (SeqSero1) and Multilocus Sequence Typing (MLST) with 813 and 1624 Salmonella isolates, respectively, and Robertson et al. (2018) evaluated accuracy of serotype prediction using SISTR and MLST using 42400 genomes deposited in the sequence read archive (SRA). Yachison et al. (2017) reported unambiguous serotype determination of $89.7 \%$ of isolates with SISTR, but only $54.1 \%$ of isolates using SeqSero1. In this study, authors considered "inconclusive" and "incongruent" matches to be successful increasing performance scores to 94.8, 88.2, and $88.3 \%$ of the isolates tested using SISTR, SeqSero, and MLST, respectively. Uelze et al. (2020) report accuracies for unambiguous serovar identification of $94,87,81$, and $79 \%$ for SISTR, SeqSero2, SeqSero1, and MLST, respectively. Higher accuracies in this study 
may be due to corrections due to repeated serological analyses for isolates where in silico predictions were incongruent with initial serotypes (Uelze et al., 2020). Finally, in the large-scale study, conducted by Robertson et al. (2018), unambiguous matches were found for $91.9 \%$ and $87.51 \%$ of isolates using SISTR and MLST, respectively. These studies not only used much larger data sets for their comparisons but also included a number of serotypes not found in this study, and that of Uelze et al. (2020) and Robertson et al. (2018) included S. enterica subspecies II to IV.

\subsubsection{Prediction of AMR Based on WGS}

Due to the increasing importance of AMR surveillance, numerous computational approaches and databases are currently being applied for in silico prediction of AMR based on WGS data, and these tools are continually improving and evolving. We evaluated seven combinations of tools and databases (Table 2.1) and found that all performed equally well with accuracies of $\geq 99 \%$ for most tool-database combinations for the set of Salmonella investigated in this study, except for the prediction of SXT resistance using KMA which had an accuracy of $98.2 \%$, and the prediction of streptomycin resistance that had an overall accuracy of $94.6 \%$ using all computational tools (Table 2.6). We were unable to determine why KMA with ResFinder database and assembled genomes provided a false negative result for $d f r A 15$ in these isolates as the same gene/allele is present in both the ResFinder and NCBI databases. In addition, CARD-RGI was also able to detect $d f r A 15$ in these assembled genomes. Analysis of assemblies using KMA with the NCBI AMR database detected these genes in the three isolates, as did analysis of raw-read data using KMA and SRST2. With the exception of 
$d f r A 15$, we did not observe further differences in performance among resistance gene databases, likely due to the extensive overlap among them, nor with assemblyindependent versus assembly-dependent analyses.

CARD RGI and the ResFinder WebTool were accessible through web interfaces using databases provided with the tools (McArthur et al., 2013; Zankari, 2014). Use of the SRST2 and KMA tools enabled more flexibility in database selection. Where SRST2 requires specific database formatting as per the developers' instructions, KMA allows very fast database indexing without requiring clustering and specific header reformatting. The CARD-RGI results were more extensive than the other tools as they also included multiple hits for efflux pumps and membrane channel proteins that have been found to confer resistance to some antibiotics. These proteins are often chromosomally encoded, typically involved in normal cellular functions, require additional genes and regulators to function, and may be species specific. Thus, the presence of these genes may not be informative for the surveillance of acquired ARGs and may require additional expertise for data interpretation.

\subsubsection{Requirements for WGS-Based ARG Detection}

There is limited discussion in the literature as to the sequence quality and genome coverage required to accurately detect ARGs using these tools. Poor sequence quality and low coverage could result in errors during assembly and removal of portions of sequence data. ARG-detection tools requiring assembled genomes risk missing a gene if it is split over multiple contigs (Clausen et al., 2016). Conversely, approaches using Bowtie2 for analyses of raw-read data risk reporting false positives due to contaminating agents in 
addition to cases where a gene may be fragmented due to insertion of another gene (Clausen et al., 2016).

Using a cutoff of $98 \%$ for target-gene identity, ARGs were not always detected at 20X genome coverage (Figure 2.1). However, lowering target-gene identity to the default cutoff of $90 \%$ gene identity, currently suggested for most in silico ARG detection programs, allowed for detection of closely related and novel alleles resulting in 100\% gene identification at 15 and 20X for most genes (Figure 2.1). Some of the aminoglycoside genes were correctly identified at $>100 \%$ of expected for coverage of 5 to $20 \mathrm{X}$ (Figure 2.1A). This is likely due to multiple isolates $(\mathrm{n}=10)$ encoding multiple alleles and/or copies of the gene at $\geq 80 \%$ identity, thereby resulting in a higher number of positive hits (confirmed using KMA on raw-reads, data not shown). In contrast, lower percent identification sometimes occurred for genes that matched closely to multiple alleles as KMA uses a scoring scheme in order to ensure the best matching template is selected, and prevent reporting of false positives, which may have resulted in $k$-mer matches to alternate alleles and under-reporting of genes at lower coverage levels (Figure 2.1) (Clausen et al., 2018). Overall, these results suggest a minimum coverage requirement of 15-20X for bacterial isolate WGSs for accurate AMR predictions, and that deeper sequencing in conjunction with lower gene identity cutoffs may improve ARG detection. In addition, ARG analysis of novel or rare bacterial species or strains via WGS may benefit from altering gene identity cutoffs in order to detect new alleles and closely related or novel resistance genes.

We considered the possibility that ARG detection may be more sensitive using raw-read sequences as this would alleviate errors arising from repeat regions and 
assembly of contaminating agents impacting genome assembly as has been observed in other studies (Carrillo et al., 2016; Clausen et al., 2016; Low et al., 2019). Assembly tools were found to have an impact on the ability to detect ARGs, particularly at low genome coverage where percentages of correctly identified genes were significantly lower in SKESA-assemblies. At higher coverage levels the use of raw-reads and both assembly types for ARG detection gave similar results for all genes tested (Figure 2.2). In contrast to SPAdes, SKESA is designed to be more conservative producing assemblies with high base level accuracy and avoids assembly of potentially questionable sequences (Souvorov et al., 2018).

In the coverage-sampled assembled dataset, the streptomycin resistance gene strA was only detected in $10 \%$ of OLC2568 assemblies but found in most of the raw-read files (Table 2.3, Figure 2.2). These results suggest identification of non-functional genes is more likely to occur when using raw-read sequence data for gene detection. In this case the fragmentation of strA by an inserted $d f r A 14$ gene in isolate OLC2568 did not affect AMR phenotype predictions as this isolate also harbored a full-length $\operatorname{str} B$ phosphotransferase.

Overall, ARG detection accuracy for isolate sequences appears to depend on sequence quality and the gene being investigated. Furthermore, if sequence coverage is greater than 15X, assembly-methods have minimal impact on ARG detection (Figure 2.2).

\subsubsection{Single nucleotide variations (SNVs) conferring AMR}


Antimicrobial resistance can be achieved through both acquisition of resistanceconferring genes and genetic adaptation through mutations (Nishimura et al., 2007; Okamoto et al., 2007; Wong et al., 2011; Mikheil et al., 2012; Lázár et al., 2013; Blair et al., 2015). Amino acid substitutions resulting in NAL resistance (NaIR) have been well documented in Salmonella and Escherichia coli (Ruiz et al., 1997; Piddock et al., 1998; Hakanen et al., 1999; Reche et al., 2002; Ruiz, 2003; Cooper et al., 2015, 2016; Knowles et al., 2015). Consistent with the literature, we identified two isolates with nonsynonymous mutations in the gyrA gene (Table 2.2) which correlated with observed phenotypic NAL and intermediate CIP resistance in these isolates (Table S2.2). Both isolates harbored non-synonymous mutations at serine 83 , which is known to be important for quinolone resistance (Piddock et al., 1998; Ruiz, 2003).

All of the ARG prediction tools failed to detect genes conferring streptomycin resistance in two strains and gentamycin resistance in one strain (Table 2.6, Table 2.7, Table S2.2). The three main mechanisms of aminoglycoside resistance include antimicrobial inactivation by aminoglycoside modifying enzymes, ribosome modification, and decreased membrane permeability (Lázár et al., 2013). Mutations resulting in lack of methylation of the $16 \mathrm{~S}$ rRNA have been found to result in STR resistance in Escherichia coli, Mycobacterium tuberculosis, Bacillus subtilis, and Salmonella spp. (Nishimura et al., 2007; Okamoto et al., 2007; Wong et al., 2011; Mikheil et al., 2012). This loss of methylation has been associated with mutations and/or deletions in the ribosomal small subunit methyltransferase G gene $r \operatorname{sm} G$ (formerly gidB) (Nishimura et al., 2007; Okamoto et al., 2007; Wong et al., 2011; Mikheil et al., 2012) which were not observed in the resistant OLC2536, OLC2626, or OLC2644. 
Failure to predict aminoglycoside resistance may also be due to mutations within efflux-related proteins that have not yet been documented (Lázár et al., 2013; GarneauTsodikova and Labby, 2016). Comparison of phenotypically resistant Salmonella ser. Muenchen (OLC2626) and resistant Salmonella ser. Heidelberg (OLC2644) to phenotypically sensitive isolates of the same serovars found a low number of SNVs. Although a few nonsynonymous mutations and a nonsense mutation were detected in comparison of the Heidelberg isolates, no obvious cause for STR-resistance in this isolate could be determined.

A study on the evolution of antibiotic hypersensitivity in E. coli conducted by Lázár et al. (2013) reported 44\% of collateral-sensitivity interactions involved resistance to aminoglycosides. Genetic analyses of hypersensitive mutants identified genes involved in membrane potential including the respiratory electron transport chain (ETC) Nuo (NADH:ubiquinone oxidoreductase) protein complex (Lázár et al., 2013). This is not surprising as aminoglycosides require respiration for uptake and aminoglycoside resistance has been linked to decreased membrane permeability (reviewed by GarneauTsodikova and Labby, 2016). Of the isolates in this study with non-synonymous mutations in nuoF only the Anatum var 15+ strain (OLC2536) and $S$. ser. Anatum isolates from the OLC-CFIA culture collection with L45R mutations presented with phenotypic STR resistance (Table 2.2). Collectively this suggests a role for the nuoF L45R mutation in STR-resistance. Further investigation of this mutation in $n u o F$ is currently being conducted to determine whether membrane potential and efflux activity are reduced and if the detected SNV in $n и о F$ plays a role in this decreased potential and STR resistance. 
Blocking of the ubiquinone biosynthesis pathway results in a defect in electron transport and aerobic respiration which has been found to increase aminoglycoside resistance (Paradise et al., 1998; Li et al., 2016). Mutations in $u b i F$ have been shown to produce pleiotropic E. coli phenotypes resistant to STR and GEN (Soballe and Poole, 1999). Similarly, Li et al. (2016) found mutations in genes $u b i E$ and $p r f B$, associated with STR induction of a small colony variant (SCV) phenotype, resulted in two- to four- fold increases in STR MIC of isolates. BLAST analyses of the coenzyme Q redox gene $u b i E$ found no frameshift or non-synonymous mutations in isolates from this study. Multiple non-synonymous mutations were detected in $u b i F$, and two isolates exhibited V165I mutations in $\operatorname{prfB}$ (Table 2.2); however, no obvious change in MIC was observed to result from these mutations.

Frequently, identification of SNV-based resistance requires alignment of protein sequences for the identification of non-synonymous mutations in regions of interest. In cases of novel SNV-based resistance, comparison to closely related isolates may be required to determine possible resistance-conferring mutations. This is not always possible when a closely related isolate is unavailable. Additional investigations into SNV-based mutations that result in evolution of AMR would be useful not only for determining novel SNV-based resistances, but also classes of genes that are associated with the evolution of AMR (Lázár et al., 2013). Continuing research is needed to identify new genetic factors conferring resistance to improve accuracy. The development of more comprehensive curated databases of SNV-based mutations conferring AMR in different pathogenic bacterial species will enable more reliable detection of SNV-based AMR in WGS datasets. 


\subsubsection{Activation of STR Resistance in Salmonella spp. Occurs in Minimal Media}

Similar to the results of Tyson et al. (2016) and Pornsukarom et al. (2018), discrepancies were observed for genotypic prediction of STR resistance even after broth and agar microdilution testing. A study by Koskiniemi et al. (2011) found activation of a chromosomally encoded adenyl transferase ( $\operatorname{add} A)$ combined with mutations affecting the electron transport chain (ETC) resulted in increased STR resistance. Their work shows that while growth in rich medium (such as LB or BHI) resulted in a phenotypically sensitive isolate, growth in minimal media or mutations that impaired the ETC resulted in conversion to a small colony variant (SCV) and activation of the chromosomal aadA gene conferring STR resistance. The subset of Salmonella isolates tested in MH, M9, and LB media all exhibited an extremely high resistance to STR in M9 with the exception of OLC2542 which appeared to have impaired growth in minimal media (Table 2.8). Growth of STR-resistant OLC2536 in M9 was similar to both STR -sensitive and resistant comparator isolates. However even without harboring acquired STR-resistance genes OLC2536 exhibited growth at high concentrations of STR in rich media (LB and $\mathrm{MH}$ ), comparable to other STR-resistant isolates, suggesting that ETC mutations in this strain may be conferring STR-resistance as described by Koskiniemi et al. (2011).

\subsection{Conclusion}

Relationships between Salmonella serotype and AMR profile can indicate the possible source of an isolate. This AMR-serotype relationship is useful for epidemiological and outbreak investigations. While identification and resistance determination of bacteria is critical for guiding therapeutic approaches in treating 
infections, use of genomic approaches has the added benefit of providing data for surveillance purposes. We have shown here that in silico tools predicting Salmonella serotypes and AMR-phenotypes are highly accurate. In fact, in this study genomic prediction of AMR was more accurate than phenotypic results. Similarly, genome-based serotype determination may be more informative than laboratory approaches for clustering genetically related isolates, particularly in cases where somatic and flagellar antigens are not expressed. However, there are some caveats - namely the importance of sequence coverage and assembly method, the involvement of chromosomal SNPs in mutations conferring resistance, and the role of the environment on resistant phenotypes as this could impact expression of genes conferring resistance. A nuoF mutation amongst STR-resistant Salmonella ser. Anatum var. 15+ strains was noted; however, the precise mechanism of aminoglycoside-resistance in three strains with no identifiable ARGs remains uncertain and indicates continuing research is needed to catalog the molecular basis of resistance mechanisms. Development and curation of high-quality verified datasets is critical for assessing performance of new pipelines/tools for WGS-analysis of pathogens. This study provides an easily accessible, verified Salmonella enterica data set containing both sensitive and resistant isolates of different serotypes for validation of in silico tools for both serotype- and AMR-determination. 


\section{Chapter 3: Detection of carbapenem-resistance genes in bacteria isolated from wastewater in Ontario}

A version of this chapter has been accepted for publication in FACETS -

Microbiology, and is currently undergoing proofing by the FACETS editorial office:

Cooper, A. L., Carter, C., McLeod, H., Wright, M., Sritharan, P., Tamber, S., Wong, A., Carrillo, C. D. and B. W. Blais. (accepted 2021). Detection of carbapenem-resistance genes in bacteria isolated from wastewater in Ontario. FACETS. 


\subsection{Abstract}

Bacterial carbapenem resistance is a major public health concern since these antimicrobials are often the last resort to treat serious human infections. To evaluate methodologies for detection of carbapenem resistance, carbapenem-tolerant bacteria were isolated from wastewater treatment plants in Toronto, Ottawa, and Arnprior. A total of 135 carbapenem-tolerant bacteria were recovered. PCR indicated the presence of carbapenem hydrolysing enzymes $\operatorname{KPC}(n=10), \operatorname{GES}(n=5), \operatorname{VIM}(n=7)$, and IMP $(n=1)$, and $\beta$-lactamases TEM ( $n=7)$, PER $(n=1)$, and OXA-variants $(n=16)$. A subset of 46 isolates were sequenced and analysed using ResFinder and CARD-RGI. Both programs detected carbapenem resistance genes in 35 sequenced isolates and AMR genes (ARGs) conferring resistance to multiple class of other antibiotics. Where $\beta$-lactamase resistance genes were not initially identified, lowering the thresholds for ARG detection enabled identification of closely related $\beta$-lactamases. However, no known carbapenem resistance genes were found in seven sequenced Pseudomonas spp. isolates. Also of note was a multi-drug-resistant Klebsiella pneumoniae isolate from Ottawa, which harboured resistance to seven antimicrobial classes including $\beta$-lactams. These results highlight the diversity of genes encoding carbapenem resistance in Ontario, and the utility of WGS over PCR for ARG detection where resistance may result from an assortment of genes.

\subsection{Introduction}

The global increase of antimicrobial resistance (AMR) in clinically-relevant microorganisms is becoming critical. AMR genes (ARGs) are of particular concern when encoded by mobile genetic elements (MGE) such as plasmids and transposons which 
have been shown to transfer ARGs to pathogenic bacteria (Noble et al., 1992; Davison, 1999; von Wintersdorff et al., 2016). The potential for transfer of genetic material within bacterial communities via conjugation and transformation increases the importance of studying and monitoring clinically significant ARGs that may be acquired by pathogenic organisms. Of particular concern is the rise in resistance to last-resort antibiotics, such as carbapenems, in microbial communities (Bush, 1999; Mulvey et al., 2003; van Duin and Doi, 2016).

Increasing levels of resistance to carbapenem antimicrobials has become a major public health concern due to their importance in human medicine and lack of development of new antimicrobials. Most concerning are infections due to carbapenemresistant Enterobacteriaceae (CRE) strains which are associated with higher morbidity and mortality rates (Chang et al., 2011; Ben-David et al., 2012; Esterly et al., 2012). Included in this group of bacteria are many species relevant to both the food industry and clinic such as Escherichia coli and Klebsiella spp. CRE have been isolated from healthy human carriers, food-producing animals, and food products around the globe (Nordmann et al., 2011).

Carbapenems belong to the $\beta$-lactam class of antimicrobials, a broad-spectrum class of agents that contain a four-membered $\beta$-lactam ring. Carbapenems include the antibiotics doripenem, ertapenem, imipenem, and meropenem (Codjoe and Donkor, 2017). They are typically reserved for treatment of the most serious infections caused by multidrug-resistant pathogens as they are not susceptible to common $\beta$-lactam resistance mechanisms (Bush, 2010; Codjoe and Donkor, 2017). 
Bacterial resistance to $\beta$-lactams typically occurs through the production of $\beta-$ lactamase enzymes that hydrolyze the $\beta$-lactam ring. $\beta$-lactamases may be grouped according to protein homology (Ambler molecular classification) and/or based on hydrolysis and inhibition profiles (Bush-Jacoby-Medeiros functional classification), resulting in derivatives such as penicillinases, cephalosporinases, carbenicillinases and carbapenemases. Different types of $\beta$-lactamases inactivate different $\beta$-lactam antimicrobials. The extended spectrum $\beta$-lactamases (ESBLs) are a group of enzymes that break down antibiotics of the penicillin and cephalosporin classes (Shaikh et al., 2015). Carbapenemases are a versatile family of the $\beta$-lactamases as they are able to hydrolyze almost all hydrolysable $\beta$-lactams including penicillins, cephalosporins, and carbapenems (Queenan and Bush, 2007). As carbapenemases are $\beta$-lactamases, they belong to one of two molecular families distinguished based on the active site. Molecular class A and D molecules contain a serine-based active site while molecular class B, also known as metallo- $\beta$-lactamases (MBLs), contain zinc (Queenan and Bush, 2007; Bush and Jacoby, 2010). Originally carbapenemases were thought to be species-specific chromosomal $\beta$-lactamases, however many of these enzymes including IMP and KPC have subsequently been identified in diverse species on plasmids (Queenan and Bush, 2007; Almeida et al., 2013).

A number of $\beta$-lactamases and ESBLs belonging to non-carbapenemase classes have also exhibited low-level carbapenem-hydrolyzing activity and can confer carbapenem resistance to some extent (Jacoby, 2009; Bonnin et al., 2011). Overall, three major mechanisms resulting in bacterial carbapenem resistance have been described: the production of carbapenemase(s), production of a $\beta$-lactamase or ESBL coupled with 
porin loss or modification, and efflux (Jacoby et al., 2004; Jacoby, 2009; Hao et al., 2018).

Phenotypic confirmation of carbapenemase production is based on detection of carbapenemase diffusion or inhibition of carbapenemase activity (Bartolini et al., 2014). Detection of diffusible carbapenemases can be evaluated using the Modified Hodge Test (MHT), however interpretation of results is subjective and may include false -positive and -negative results (Girlich et al., 2012; Pierce et al., 2017; Tamma et al., 2017). Similarly, the Carba NP test suffers from subjective results, poor sensitivity, and the requirement of specialized reagents (Pierce et al., 2017; Tamma et al., 2017). Measuring inhibition of carbapenemase activity is conducted using EDTA and dipicolinic acid against MBLs, boronic acid to inhibit Ambler class A enzymes, and cloxacillin against ampicillinases (Bartolini et al., 2014). Unfortunately most of the available methods to detect carbapenemase production were designed primarily for Enterobacteriaceae, involve time-consuming protocols, are subjective, not very sensitive/specific, and many require specialized reagents (Pierce et al., 2017).

Evidence has shown that human activities facilitate the emergence and dissemination of AMR bacteria in the environment (Li et al., 2015; Freeman et al., 2018). This is of great concern as bacteria, possibly containing ARGs, are capable of persistence and spreading throughout the environment (Anderson et al., 2005). Wastewater treatment plants (WWTPs) are environmental hot spots for ARG dissemination, as these sites typically have extremely high bacterial loads from human sources combined with subinhibitory concentrations of antimicrobials discharged from household, hospital, agricultural, and industrial human sources (Berendonk et al., 2015; Li et al., 2015). 
AMR surveillance programs such as the European program DARE (Detecting evolutionary hot spots of Antibiotic Resistance in Europe) have suggested that resistance testing should focus on resistance determinants that are clinically relevant, prevalent in the environment, associated with mobile genetic elements and have the potential to be acquired by horizontal gene transfer (Berendonk et al., 2015). Candidate genes frequently occurring in various environments impacted by anthropogenic activities include the $\beta$ lactamases $b l a_{\mathrm{CTX}-\mathrm{M}}, b l a_{\mathrm{TEM}}, \mathrm{MBL} b l a_{\mathrm{NDM}-1}$, carbapenemases $b l a_{\mathrm{KPC}}$ and $b l a_{\mathrm{VIM}}$ (among others) (Jacoby, 2009).

Wastewater is increasingly used for surveillance of AMR as it contains an abundance of bacteria derived from anthropogenic sources and elevated levels of ARGs (Li et al., 2015; Hembach et al., 2017; Muurinen et al., 2017; Ng et al., 2017; Freeman et al., 2018; Hutinel et al., 2019; Pärnänen et al., 2019; Aarestrup and Woolhouse, 2020; Sims and Kasprzyk-Hordern, 2020). Effective surveillance programs require rapid methods capable of processing large numbers of samples. We therefore sought to assess methodologies for detection and enumeration of bacterial carbapenem-resistance genes using sewage influent from different WWTPs, as carbapenems are clinically very important for treatment of difficult infections, and wastewater influent is likely to harbour carbapenem-tolerant bacteria. 


\subsection{Materials and Methods}

\subsubsection{Sampling}

Wastewater (influent) samples (1 L) were collected from Arnprior, a small agricultural city (March 3, 2017); Ottawa, a mid-sized city with light industry (March 10, 2017); and Toronto, a major industrialized city (March 17, 2017). Samples were received on ice at the Ottawa Laboratory (Canadian Food Inspection Agency) where they were filtered and plated within 24 hours of collection.

\subsubsection{Microbiological analysis}

Carbapenem-resistance in this study was defined as growth on agar containing $\geq 4$ $\mu \mathrm{g} / \mathrm{ml}$ meropenem for Enterobacteriaceae, $\geq 8 \mu \mathrm{g} / \mathrm{ml}$ for Stenotrophomonas maltophilia, and $\geq 16 \mu \mathrm{g} / \mathrm{ml}$ for other non-Enterobacterales including Pseudomonas spp. (but not $P$. aeruginosa), as recommended by CLSI guidelines (CLSI, 2020). Carbapenem-tolerance was defined as growth on media containing $4 \mu \mathrm{g} / \mathrm{ml}$ for $S$. maltophilia and Pseudomonas spp. (CLSI, 2020).

Water analyses were conducted as per the method of Zurfluh et al (Zurfluh et al., 2013). Briefly, $250 \mathrm{ml}$ of each water sample was filtered through a series of $0.80 \mu \mathrm{m}$ and $0.22 \mu \mathrm{m}$ sterile membrane filters (S-Pak Membrane Filters, MilliporeSigma, Ottawa, Canada). For each sample all filters were aseptically transferred to a single sterile stomacher bag containing $25 \mathrm{ml}$ modified tryptone soya broth (mTSB) (Oxoid, Ottawa, Canada). Filters were rinsed by manually massaging stomacher bags for 5 minutes to release the collected bacterial cells. An aliquot from each filter rinse was serially diluted 
in sterile phosphate buffered saline (PBS), and dilutions were plated in triplicate on MacConkey agar (MAC) (Sigma, Ottawa, Canada) containing $4 \mu \mathrm{g} / \mathrm{ml}$ meropenem (USP, Maryland USA) to select for carbapenem-tolerant Gram-negative bacteria. Plates were incubated at $37^{\circ} \mathrm{C}$ for 24 hours followed by storage at $4^{\circ} \mathrm{C}$ until further analysis.

Fifty (Toronto), 51 (Ottawa), and 34 (Arnprior) colony forming units (CFU) were randomly selected from spread plates for purification via re-streaking on MAC containing $4 \mu \mathrm{g} / \mathrm{ml}$ meropenem using a sterile loop. Purified isolates were used for further AMR characterization and $\beta$-lactamase ARG detection as described below.

\subsubsection{Carbapenemase production confirmation}

All isolates were tested for carbapenemase-production using the modified Hodge test (MHT) according to CLSI guidelines (Clinical and Laboratory Standards Institute, 2013). A 0.5 MacFarland suspension of ATCC 25922 Escherichia coli was diluted 1:10 in saline, and a lawn was spread on MHA and left to dry for 3 minutes. A $10 \mu \mathrm{g}$ meropenem disc (Thermo Scientific Oxoid, Ottawa, Canada) was placed in the centre of the agar plate. Following disc placement, a sterile swab was used to pick 3 colonies of the purified test organism and was streaked from the edge of the disc to the edge of the agar plate in a straight line. Plates were incubated at $37^{\circ} \mathrm{C}$ overnight. If the test organism allowed enhanced growth of the ATCC 25922 lawn towards the disc (into the inhibition zone producing a clover-leaf shape) it was considered to be a carbapenemase producer. 


\subsubsection{DNA Extraction}

For each purified isolate, one colony was transferred to $1.2 \mathrm{~mL}$ of Mueller-Hinton broth (MHB) (Themo Scientific Oxoid, Hants UK) containing $4 \mu \mathrm{g} / \mathrm{mL}$ meropenem using a sterile loop and incubated at $36^{\circ} \mathrm{C}$ overnight. Genomic DNA was isolated from overnight broth cultures using the Promega Maxwell 16 Cell DNA purification kit (Promega, WI, USA). For each sample, $400 \mu \mathrm{L}$ of broth culture was added to a Maxwell SEV cartridge and the remaining $800 \mu \mathrm{L}$ of culture was mixed with an equal volume of $30 \%$ glycerol and frozen at $-80^{\circ} \mathrm{C}$ for long term storage. Extracted DNA was stored at $20^{\circ} \mathrm{C}$.

\subsubsection{Characterization of $\boldsymbol{\beta}$-lactamases}

Genomic DNA was diluted 1:10 (to a concentration of approximately $1-4 \mathrm{ng} / \mu \mathrm{l}$ ) and utilized in PCR reactions as described previously (Bogaerts et al., 2013). Twenty-one primer sets (Integrated DNA Technologies, IL, USA) were used to screen for select $\beta$ lactamase, ESBL, and carbapenemase resistance genes (Table 3.1). Primers for the OXA PCR were designed in house as follows: (1) nucleotide sequences for multiple alleles of each gene were downloaded from the Center for Genomic Epidemiology (CGE) ResFinder database (Accessible at:

https://bitbucket.org/genomicepidemiology/resfinder_db, accessed September 2016); (2) alleles for each gene were aligned and analysed for conserved sequence regions; (3) conserved regions were input to NCBI Primer-BLAST to design target-specific primers for use in PCR (Ye et al., 2012). The $25 \mu \mathrm{L}$ reaction mixtures contained $2 \mu \mathrm{L}$ of diluted DNA extract, $12.5 \mu \mathrm{L}$ of $2 \mathrm{X}$ TopTaq Mastermix (Qiagen, USA), and $0.2 \mu \mathrm{M}$ of each 
primer (with the exception of the CARBA multiplex PCR which contained $0.6 \mu \mathrm{M}$ of bla $_{\mathrm{IMP}}$ primers and $0.2 \mu \mathrm{M}$ of other primers, Table 3.1). PCR was performed using a BioRad C1000 Touch Thermal Cycler (BioRad, CA, USA) with the following conditions: $15 \mathrm{~min}$ at $95^{\circ} \mathrm{C}, 30$ cycles of $30 \mathrm{~s}$ denaturation at $94^{\circ} \mathrm{C}, 90 \mathrm{~s}$ annealing at $57^{\circ} \mathrm{C}$, and $90 \mathrm{~s}$ elongation at $72^{\circ} \mathrm{C}$, followed by a final elongation at $72^{\circ} \mathrm{C}$ for $10 \mathrm{~min}$. PCR amplicons were analysed using capillary electrophoresis on the Qiaxcel DNA Screening system (Qiagen, USA) with QX alignment markers 15/3000 bp, and QX DNA size marker $100-2500 \mathrm{bp}$.

\subsubsection{Species Identification by MALDI-TOF}

The species identity of each isolate was determined through the use of matrixassisted laser desorption/ionization time of flight mass spectrometry (MALDI-TOF). Briefly, colonies were streaked onto tryptic soy agar and incubated overnight at $37^{\circ} \mathrm{C}$. One bacterial colony was applied on duplicate spots of a Biotarget plate, air dried, and overlaid with freshly prepared $\alpha$-cyano-4-hydroxy-cinnamic acid (HCCA) matrix. Mass spectra were obtained using a MALDI Biotyper mass spectrometer (Bruker Daltonic, Bremen, Germany) and compared to the Biotyper taxonomy database using the MALDI Biotyper 3.1 software. Isolates that received score values of 2.3 to 3 were identified to the species level. Scores between 2 to 2.299 corresponded to isolates identified to the genus level. 
Table 3.1. Primers used for $\beta$-lactamase, ESBL, and carbapenemase gene detection

\begin{tabular}{|c|c|c|c|c|c|}
\hline PCR Name & Targeted gene & & Primer Sequence (5' to $\left.3^{\prime}\right)^{a}$ & $\begin{array}{l}\text { Amplicon } \\
\text { Size }\end{array}$ & Reference \\
\hline \multirow[t]{2}{*}{ BactQuant $^{\mathrm{b}}$} & \multirow[t]{2}{*}{$16 S$} & $\mathrm{~F}$ & CCTACGGGDGGCWGCA & \multirow[t]{2}{*}{$466 \mathrm{bp}$} & \multirow{2}{*}{$\begin{array}{l}\text { (Liu et al., } \\
\text { 2012) }\end{array}$} \\
\hline & & $\mathrm{R}$ & GGACTACHVGGGTMTCTAATC & & \\
\hline \multirow{10}{*}{ CARBA } & \multirow{2}{*}{$b^{b l a} a_{\mathrm{NDM}}$} & $\mathrm{F}$ & ACTTGGCCTTGCTGTCCTT & \multirow[t]{2}{*}{$603 \mathrm{bp}$} & \multirow{2}{*}{$\begin{array}{l}\text { (Bogaerts et } \\
\text { al., 2013) }\end{array}$} \\
\hline & & $\mathrm{R}$ & CATTAGCCGCTGCATTGAT & & \\
\hline & \multirow[t]{2}{*}{ bla $_{\mathrm{VIM}}$} & $\mathrm{F}$ & TGTCCGTGATGGTGATGAGT & \multirow[t]{2}{*}{$437 \mathrm{bp}$} & \multirow{2}{*}{$\begin{array}{l}\text { (Bogaerts et } \\
\text { al., 2013) }\end{array}$} \\
\hline & & $\mathrm{R}$ & ATTCAGCCAGATCGGCATC & & \\
\hline & \multirow{2}{*}{$b l a_{\mathrm{KPC}}$} & $\mathrm{F}$ & TCGCCGTCTAGTTCTGCTGTCTTG & \multirow[t]{2}{*}{$353 \mathrm{bp}$} & \multirow{2}{*}{$\begin{array}{l}\text { (Bogaerts et } \\
\text { al., 2013) }\end{array}$} \\
\hline & & $\mathrm{R}$ & ACAGCTCCGCCACCGTCAT & & \\
\hline & \multirow[t]{2}{*}{ bla $_{\text {OXA-48 }}$} & $\mathrm{F}$ & ATGCGTGTATTAGCCTTATCG & \multirow[t]{2}{*}{$265 \mathrm{bp}$} & \multirow{2}{*}{$\begin{array}{l}\text { (Bogaerts et } \\
\text { al., 2013) }\end{array}$} \\
\hline & & $\mathrm{R}$ & CATCCTTAACCACGCCCAAATC & & \\
\hline & \multirow{2}{*}{$b l a_{\mathrm{IMP}}$} & $\mathrm{F}$ & ACAYGGYTTRGTDGTKCTTG & \multirow[t]{2}{*}{$387 \mathrm{bp}$} & \multirow{2}{*}{$\begin{array}{l}\text { (Bogaerts et } \\
\text { al., 2013) }\end{array}$} \\
\hline & & $\mathrm{R}$ & GGTTTAAYAAARCAACCACC & & \\
\hline \multirow[t]{4}{*}{ MINESBL } & \multirow[t]{2}{*}{$b l a_{\mathrm{PER}}$} & $\mathrm{F}$ & AGTGTGGGGGCCTGACGAT & \multirow[t]{2}{*}{$725 \mathrm{bp}$} & \multirow{2}{*}{$\begin{array}{l}\text { (Bogaerts et } \\
\text { al., 2013) }\end{array}$} \\
\hline & & $\mathrm{R}$ & GCAACCTGCGCAATRATAGCTT & & \\
\hline & \multirow[t]{2}{*}{$b^{\prime l a} a_{\mathrm{GES}}$} & $\mathrm{F}$ & CTGGCAGGGATCGCTCACTC & \multirow[t]{2}{*}{$600 \mathrm{bp}$} & \multirow{2}{*}{$\begin{array}{l}\text { (Bogaerts et } \\
\text { al., 2013) }\end{array}$} \\
\hline & & $\mathrm{R}$ & TTCCGATCAGCCACCTCTCA & & \\
\hline \multirow[t]{6}{*}{$\mathbf{O X} \mathbf{A}^{\mathbf{c}}$} & \multirow[t]{2}{*}{ bla $_{\mathrm{OXA}-51}$} & $\mathrm{~F}$ & GACTGCACGCCGGAACTC & \multirow[t]{2}{*}{$156 \mathrm{bp}$} & \multirow[t]{2}{*}{ This study ${ }^{c}$} \\
\hline & & $\mathrm{R}$ & CAGTATCCCGAGAGCCTTGA & & \\
\hline & bla $_{\text {OXA-2 }}$ & $\mathrm{F}$ & TTTCTCTTGCCACTTTCGCG & $409 \mathrm{bp}$ & This study ${ }^{c}$ \\
\hline & & $\mathrm{R}$ & GCGTTGCCATAGTCGATTTTC & & \\
\hline & bla $_{\mathrm{OXA}-10}$ & $\mathrm{~F}$ & GCCGTCAATGGTGTCTTCGT & $544 \mathrm{bp}$ & This study $^{\mathrm{c}}$ \\
\hline & & $\mathrm{R}$ & TGACTCAGTTCCCACACCAGA & & \\
\hline POM & bla $_{\mathrm{POM}-1}$ & $\mathrm{~F}$ & GACAGCCAGGACATCCACTT & $210 \mathrm{bp}$ & This study ${ }^{\mathrm{c}}$ \\
\hline & & $\mathrm{R}$ & GCTCAGGCTGTCGCTGTAG & & \\
\hline TEM-SHV & bla $_{\mathrm{TEM}}$ & $\mathrm{F}$ & TCGCCGCATACACTATTCTCAGAATGA & $445 \mathrm{bp}$ & (Monstein $e t$ \\
\hline & & $\mathrm{R}$ & ACGCTCACCGGCTCCAGATTTAT & & al., 2007) \\
\hline & $b l a_{\mathrm{SHV}}$ & $\mathrm{F}$ & ATGCGTTATATTCGCCTGTG & $747 \mathrm{bp}$ & (Paterson et \\
\hline & & $\mathrm{R}$ & TGCTTTGTTATTCGGGCCAA & & al., 2003) \\
\hline CTX-M-U & bla $_{\mathrm{CTX-M}}$ & $\mathrm{F}$ & ATGTGCAGYACCAGTAARGTKATGGC & $593 \mathrm{bp}$ & (Mulvey et \\
\hline & & $\mathrm{R}$ & TGGGTRAARTARGTSACCAGAAYCAGCGG & & al., 2003) \\
\hline
\end{tabular}

${ }^{a}$ Degenerate bases: $\mathrm{Y}$, is a pyrimidine; $\mathrm{R}$, is a purine; $\mathrm{K}$, is a $\mathrm{G}$ or $\mathrm{T} ; \mathrm{S}$, is a $\mathrm{G}$ or $\mathrm{C}$.

${ }^{\mathrm{b}}$ The BactQuant $16 \mathrm{~S}$ primers were used as a positive control to ensure bacterial DNA was present in diluted gDNA samples.

${ }^{\mathrm{c}}$ Alleles for each gene downloaded from Center for Genomic Epidemiology ResFinder database. Nucleotide sequences of alleles were aligned, and NCBI Primer Blast used to design primers for conserved regions.

\subsubsection{Whole Genome Sequencing (WGS) and in silico ARG Detection}

Isolates from a variety of genera with at least one $\beta$-lactam resistance gene as determined by PCR were selected for sequencing. A number of PCR-negative isolates were also selected to investigate additional ARGs that may be conferring resistance.

From Toronto, Ottawa, and Arnprior 15, 17, and 14, isolates (respectively) were selected 
for sequencing. Of the selected isolates eight, eight, and one isolate(s) were selected based on PCR-positive results (Toronto, Ottawa, and Arnprior respectively). The remaining isolates sequenced from each WWTP were PCR-negative. Sequencing libraries were constructed using the Nextera XT DNA sample preparation kit (Illumina, Inc., San Diego, CA) and paired-end sequencing was performed on the Illumina MiSeq platform (Illumina Inc.) using 600 cycle MiSeq reagent kits (v3) with 6\% PhiX control. Paired-end Illumina MiSeq sequencing reads were processed with the CFIA-OLC Workflow for Bacterial Assembly and Typing (COWBAT). Source code for COWBAT can be found online: https://github.com/OLC-Bioinformatics/COWBAT. Quality of raw sequencing reads was assessed with FastQC version 0.11.8 (Andrews, 2010). Quality trimming was performed with BBDuk from BBTools version 38.22 (Bushnell, 2014) with the following parameters: trim quality of 10 and removal of reads below $50 \mathrm{bp}$ long. Error correction was performed using tadpole version 8.22 (Bushnell, 2014) in 'correct' mode with default parameters. Sequences were checked for contamination using ConFindr 0.5.0 with default parameters (Low et al., 2019). Contigs were assembled from the trimmed and error-corrected reads using SKESA version 2.3.0 with the vector percent argument disabled (Souvorov et al., 2018). Pilon version 1.22 (Walker et al., 2014)3 was used to perform one round of automatic assembly improvement. Quality was assessed with Qualimap version 2.2.2 (García-Alcalde et al., 2012; Okonechnikov et al., 2016). Multilocus sequence typing (MLST) was conducted using the Center for Genomic Epidemiology MLST-2.0 Server (Larsen et al., 2012), which utilizes MLST allele sequence and profile data from PubMLST.org. 
ARG detection in isolate sequences was conducted using both the Comprehensive Antibiotic Resistance Database Resistance Gene Identifier (CARD-RGI) tool version 5.1.0 with default settings (McArthur et al., 2013), and the Center for Genomic Epidemiology (CGE) Bacterial Analysis Pipeline webtool ResFinder version 2.1 (https://cge.cbs.dtu.dk/services/ResFinder-2.1/ last accessed 2020-02-17) (Zankari et al., 2012) with the default settings for gene identity threshold (90\%) and minimum length (60 \%) unless stated otherwise. Plasmid detection was conducted using the MOB-typer and MOB-recon tools from MOB-suite version 3.0.0 (Robertson and Nash, 2018). ARG location determination was conducted by matching the contig encoding the ARG to the matching contig output as plasmid or chromosome by MOB-recon tool.

\subsubsection{Nucleotide sequence accession numbers}

Whole-genome sequences have been deposited at DDBJ/EMBL/GenBank under bioproject PRJNA489399. Sequence read archive (SRA) accession numbers, MHT, PCR, WGS-ARG (ResFinder and CARD-RGI), and MALDI-TOF identification data can be found in Supplementary Table S3.1. MOB-typer plasmid contents for each sequence can be found in Supplementary Table S3.2. 


\subsection{Results and Discussion}

\subsubsection{Characterization of carbapenem-resistant strains recovered from WWTP}

influents

A total of 135 meropenem-tolerant strains (growth on $\geq 4 \mu \mathrm{g} / \mathrm{ml}$ meropenem) were recovered from WWTP influent samples from Toronto (50), Ottawa (51) and Arnprior (34). Genera identified included Aeromonas, Enterobacter, Klebsiella, Pandoraea, Providencia, Pseudomonas and Stenotrophomonas as determined using MALDI-TOF (Figure 3.1b, Table S3.1).

All strains were tested for carbapenemase-production using the modified Hodge test (MHT) which indicated 12, 7, and 0 carbapenemase-producing strains from Toronto, Ottawa, and Arnprior, respectively. Predicted carbapenemase producing strains included Aeromonas, Klebsiella, Pseudomonas, or Providencia genera (Table 3.2).

Table 3.2. Number of isolates confirmed by Modified Hodge Test (MHT) to be carbapenemase producers

\begin{tabular}{lcc}
\hline Genus & Total & $\begin{array}{c}\text { No. of carbapenemase- } \\
\text { producers }^{\mathbf{a}}\end{array}$ \\
\hline Aeromonas & 10 & 5 \\
Enterobacter & 1 & 0 \\
Klebsiella & 9 & 9 \\
Pandoraea & 2 & 0 \\
Providencia & 1 & 1 \\
Pseudomonas & 95 & 4 \\
Stenotrophomonas & 9 & 0 \\
\hline
\end{tabular}

${ }^{\mathrm{a}}$ Number of carbapenemase producers determined using the Modified Hodge Test. 
Figure 3.1. Identified resistance genes and genera. (Page 93) (a) Number of strains encoding PCR identified $\beta$-lactamase and carbapenemase resistance genes isolated from each wastewater treatment plant (WWTP) influent sample. Genes corresponding to coloured bars are listed in the legend. Number above bar denotes number of isolated strains encoding gene for corresponding city's sample. (b) MALDI-TOF identified bacterial genera isolated from Toronto, Ottawa, and Arnprior WWTP influent samples. Stacked bars correspond to number of isolates belonging to each genus (see legend). $\mathrm{n}$ $=46,48$, and 31 identified isolates for Toronto, Ottawa, and Arnprior respectively. Isolates that were not identified by the MALDI-TOF were excluded from calculations. 

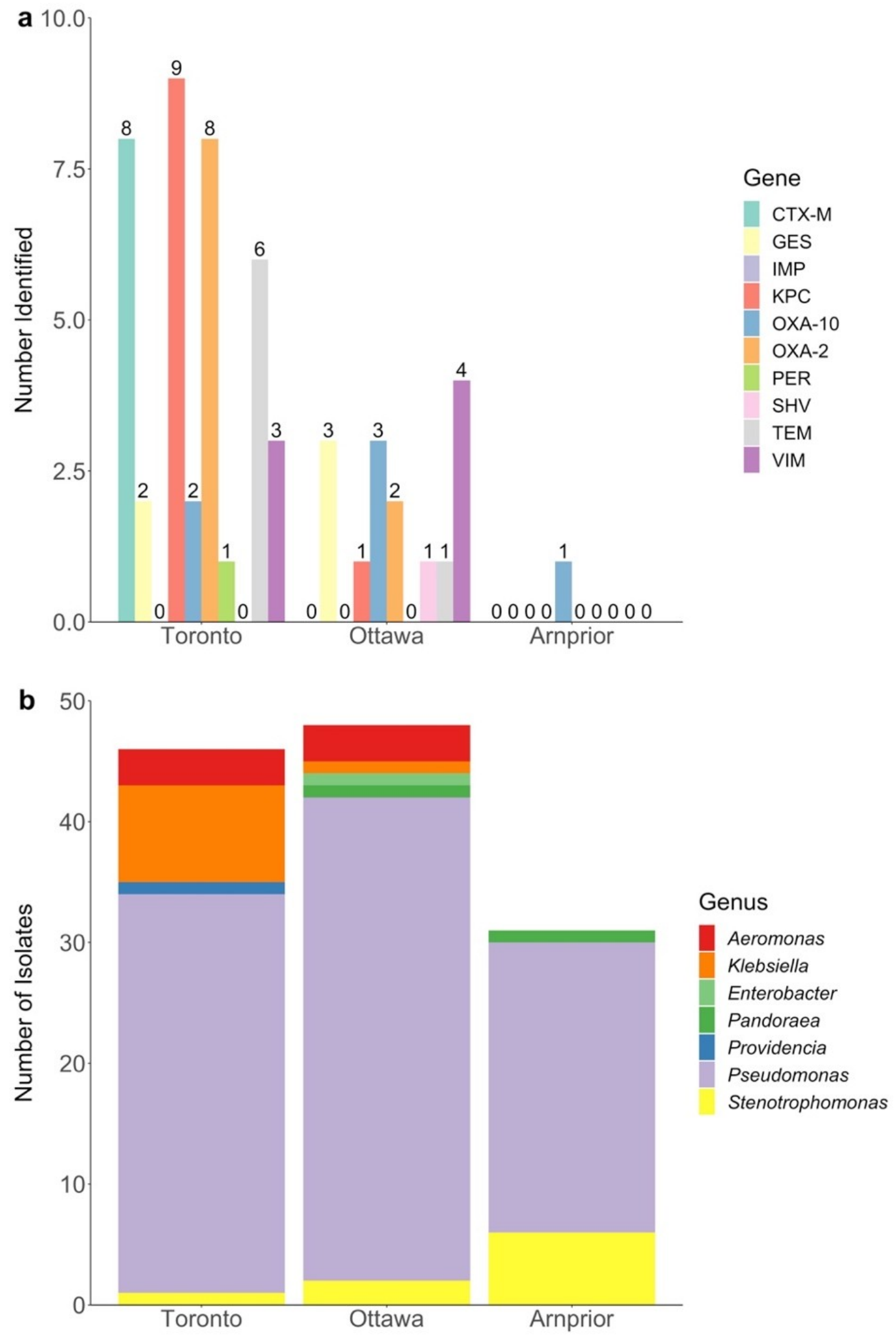


\subsubsection{Identification of Antibiotic Resistance Genes (ARGs) in Isolates found in WWTP Influents}

Strains were screened for ARGs using a panel of 21 PCR assays to identify select $\beta$-lactamase, ESBL, and carbapenemase resistance genes (Table 3.1). PCR analysis indicated the presence of $\beta$-lactamase genes $(b l a)$ with the potential to hydrolyze carbapenems in 35 strains (Figure 3.1a, Table S3.1). The majority of $\beta$-lactam-related ARGs were identified in isolates from the Toronto WWTP site, followed by Ottawa (Figure 3.1a). Isolates from Arnprior exhibited the lowest number of ARGs, and those isolates which were identified mostly belonged to species not typically considered to be AMR indicators (eg. P. fluorescens and P. putida) (Berendonk et al., 2015) (Figure 3.1).

The PCR analyses focused on $\beta$-lactamase genes reported to confer carbapenemresistance (Table 3.3) (Paterson et al., 2003; Monstein et al., 2007; Jacoby, 2009; Bush, 2010; Bonnin et al., 2011; Nordmann et al., 2011; Bogaerts et al., 2013; van Duin and Doi, 2016). Genes for class A carbapenemases are typically found on transferrable plasmids and are highly prevalent among Enterobacteriaceae, with KPC being the most prevalent (van Duin and Doi, 2016). The bla $a_{\mathrm{KPC}}$ gene was one of the most common ARGs detected by PCR among the isolates with one Klebsiella testing positive from the Ottawa site, as well as eight Klebsiella and a single Providencia from the Toronto site (Figure 3.1a). The class A carbapenemase GES enzyme was detected in two Toronto and three Ottawa Aeromonas isolates (Figure 3.1a, Figure 3.2).

The metallo- $\beta$-lactamases VIM and IMP were detected in Pseudomonas spp. isolates recovered from both Toronto and Ottawa (Figure 3.1, Figure 3.2). VIM and IMP are both Class B, metallo- $\beta$-lactamases which generally have weak $\beta$-lactamase activity 
against all $\beta$-lactams except monobactams but are able to hydrolyze carbapenems (Table 3.3) (Bush, 2010). They are frequently associated with multiple AMR determinants and have spread globally to many of the Enterobacteriaceae (Nordmann et al., 2011; Codjoe and Donkor, 2017).

Table 3.3. Summary of molecular and functional characteristics of $\beta$-lactamase genes (bla) relevant to this study

\begin{tabular}{|c|c|c|c|c|c|c|c|}
\hline $\begin{array}{l}\text { Active } \\
\text { Site }\end{array}$ & Molecular & Functional & $\begin{array}{l}\text { bla } \\
\text { Gene* }\end{array}$ & Location $^{b}$ & $\begin{array}{l}\text { Common } \\
\text { Name }\end{array}$ & Resistance & References \\
\hline \multirow[t]{8}{*}{ Serine } & \multirow[t]{2}{*}{$\mathrm{C}$} & 1 & $\begin{array}{l}\text { AmpC } \\
\text { Amp* } \\
\text { ACC } \\
\text { ACT-1 } \\
\text { CepH } \\
\text { FOX-1 } \\
\text { MIR-1 } \\
\text { ACT-28 }\end{array}$ & $\begin{array}{l}\mathrm{C} \\
\mathrm{C}, \mathrm{P} \\
\mathrm{P} \\
\mathrm{P} \\
\mathrm{C} \\
\mathrm{P} \\
\mathrm{P} \\
\mathrm{C}\end{array}$ & Cephalosporinase & $\begin{array}{l}\text { Cephalosporins, } \\
\text { cephamycins }\end{array}$ & $\begin{array}{l}\text { (Gonzalez Leiza } \\
\text { et al., 1994; } \\
\text { Bush } \text { et al., } \\
\text { 1995; Jacoby, } \\
\text { 2009; Bush, } \\
\text { 2010; } \text {;hilippon } \\
\text { et al., 2016; } \\
\text { Jousset et al., } \\
\text { 2019) }\end{array}$ \\
\hline & & $1,1 \mathrm{e}$ & $\begin{array}{l}\text { CMY-2, } \\
\text { CMY-37 }\end{array}$ & C,P & $\begin{array}{l}\text { Cephalosporinase, } \\
\text { ESAC } \beta \text { - } \\
\text { lactamase }\end{array}$ & $\begin{array}{l}\text { Cephalosporins, } \\
\text { cephamycins }\end{array}$ & (Jacoby, 2009) \\
\hline & \multirow[t]{6}{*}{ A } & $2 \mathrm{a}$ & LEN-1 & $\mathrm{C}$ & Penicillinase & Penicillins & $\begin{array}{l}\text { (Philippon et al., } \\
\text { 2016) }\end{array}$ \\
\hline & & $2 \mathrm{~b}$ & $\begin{array}{l}\text { SHV-1 } \\
\text { TEM- } \\
1,2\end{array}$ & $\begin{array}{l}\text { C,P } \\
\text { C,P }\end{array}$ & Penicillinase & $\begin{array}{l}\text { Penicillins, } \\
\text { cephalosporins }\end{array}$ & $\begin{array}{l}\text { (Jacoby, 2009; } \\
\text { Philippon et al., } \\
\text { 2016) }\end{array}$ \\
\hline & & $2 \mathrm{be}$ & $\begin{array}{l}\text { BEL-1 } \\
\text { CTX-M } \\
\text { OXY } \\
\text { PER } \\
\text { SHV-* } \\
\text { TEM-* } \\
\text { VEB }\end{array}$ & $\begin{array}{l}\mathrm{P} \\
\mathrm{C}, \mathrm{P} \\
\mathrm{C}, \mathrm{P} \\
\mathrm{P} \\
\mathrm{C}, \mathrm{P} \\
\mathrm{C}, \mathrm{P} \\
\mathrm{P}\end{array}$ & ESBL & $\begin{array}{l}\text { Extended- } \\
\text { spectrum } \\
\text { cephalosporins } \\
\text { (cefepime), } \\
\text { monobactams }\end{array}$ & $\begin{array}{l}\text { (Jacoby, 2009; } \\
\text { Philippon et al., } \\
\text { 2016) }\end{array}$ \\
\hline & & $2 \mathrm{br}, 2 \mathrm{ber}$ & $\begin{array}{l}\text { SHV_* } \\
\text { TEM-* }\end{array}$ & $\begin{array}{l}\text { C,P } \\
\text { C,P }\end{array}$ & ESBL & $\begin{array}{l}\text { Penicillins (2br), } \\
\text { extended- } \\
\text { spectrum } \\
\text { cephalosporins } \\
\text { (cefepime), } \\
\text { monobactams }\end{array}$ & $\begin{array}{l}\text { (Jacoby, 2009; } \\
\text { Philippon et al., } \\
\text { 2016) }\end{array}$ \\
\hline & & $2 c$ & $\begin{array}{l}\text { CARB-3 } \\
\text { PSE }\end{array}$ & $\begin{array}{l}\mathrm{C}, \mathrm{P} \\
\mathrm{P}\end{array}$ & Carbenicillinase & $\begin{array}{l}\text { Penicillins, } \\
\text { carbenicillin }\end{array}$ & $\begin{array}{l}\text { (Philippon et al., } \\
\text { 2016) }\end{array}$ \\
\hline & & $2 \mathrm{f}$ & GES & $\mathrm{C}, \mathrm{P}$ & Carbapenemase & Carbapenems & $\begin{array}{l}\text { (Walther- } \\
\text { Rasmussen and } \\
\text { Høiby, 2007; }\end{array}$ \\
\hline
\end{tabular}




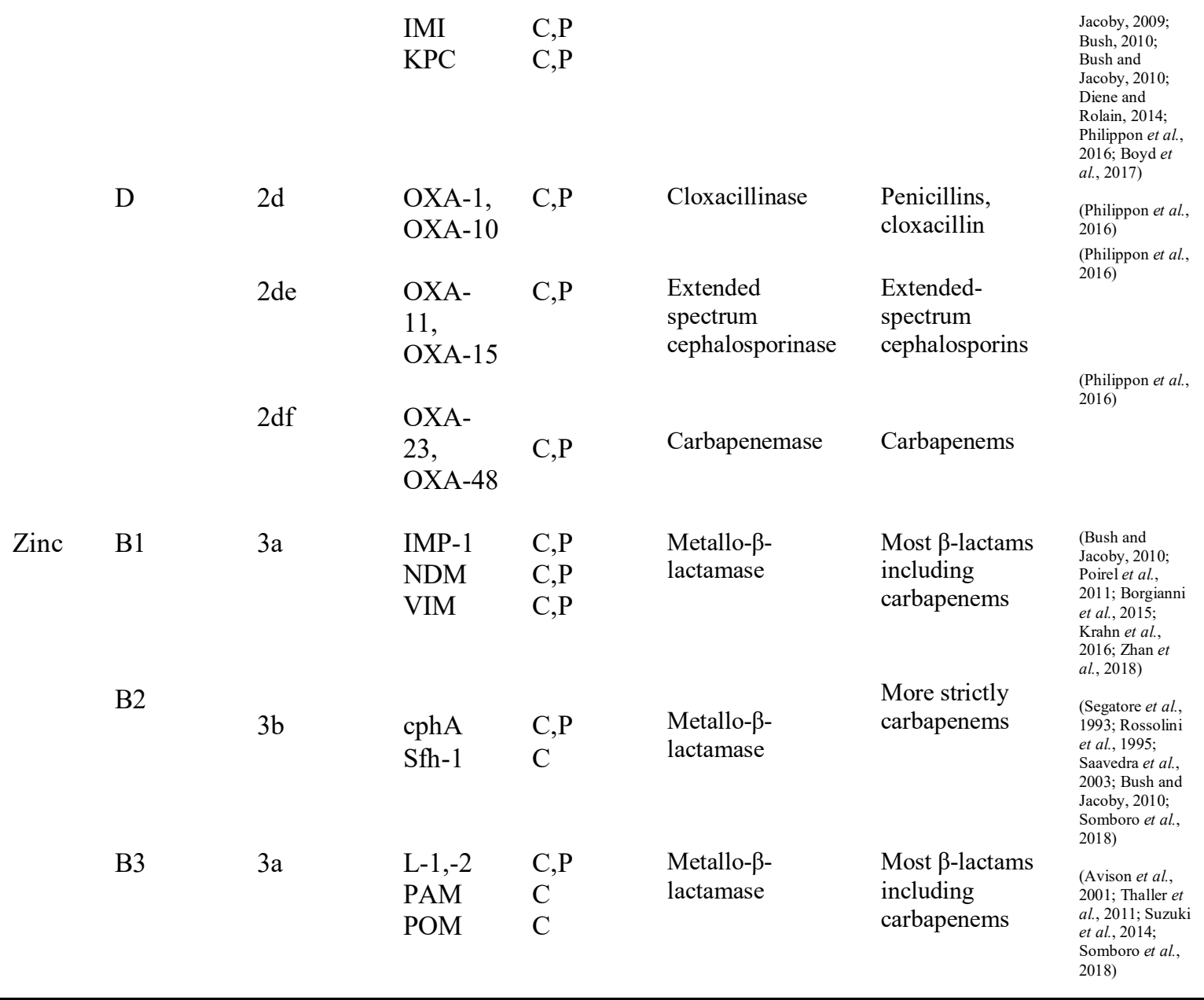

\footnotetext{
Abbreviations: Molecular, Molecular classification; Functional, functional classification; ESAC, Extended spectrum AmpC; ESBL, extended spectrum $\beta$-lactamase.

*Some gene variants exhibit broader-spectrum resistance to additional antimicrobials, therefore are in separate functional categories

ESBLs are also able to hydrolise the $4^{\text {th }}$ generation cephalosporin cefepime, whereas AmpCs are able to hydrolyse cephamycins

${ }^{a}$ Enzyme ACT-28 associated with decreased membrane permeability has been found to result in decreased carbapenem sensitivity (Jousset et al., 2019)
} 
Figure 3.2. Results of PCR and whole genome sequence analyses for $\beta$-lactam related antibiotic resistance genes (ARGs) in sequenced isolates from wastewater treatment plants in Ontario Canada. (Page 98). Data is shown for sequenced isolates from this study. Symbols: black square, positive result; white square, negative result. Coloured sidebar denotes isolate genus determined based on WGS data (see legend). *Enzymes L1 and ACT were not detected by ResFinder with default settings: genes were detected by lowering \%ID threshold to $80 \%$. **OXA-variants other than OXA-2 and OXA-10 detected via WGS. Abbreviations: WWTP, wastewater treatment plant; bla, $\beta$ lactamase; WGS, whole-genome sequence; Inc, plasmid incompatibility group predicted by MOB-suite v. 3.0.0. WGS results for non- $\beta$-lactamase-related ARGs are not shown. 


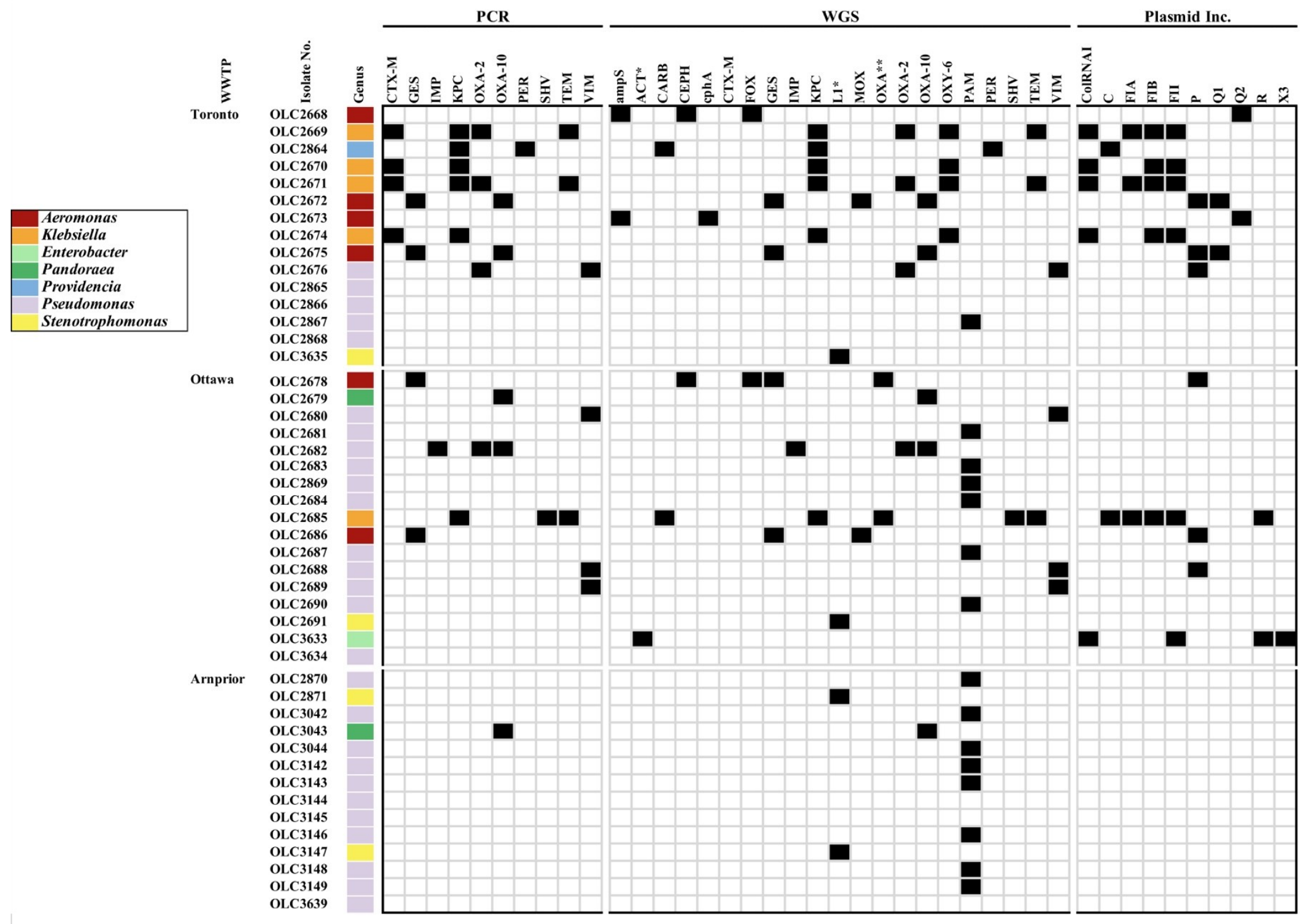


PCR analysis was also conducted to investigate non-carbapenemase class A ESBL genes (Table 3.3), of which nine isolates were predicted to harbour bla $a_{\mathrm{PER}}(\mathrm{n}=1)$ and bla $_{\text {СTX-M }}(\mathrm{n}=8)$ based on PCR results (Figure 3.1a). However, WGS analysis of four CTX-M PCR-positive isolates did not detect the $b a_{\mathrm{CTX}-\mathrm{M}}$ gene but instead detected the class A ESBL bla $a_{\mathrm{OXY}-6}$. The chromosomal class A enzymes of Klebsiella oxytoca (bla $a_{\mathrm{OXY}}$ ) have been documented as one of the $\beta$-lactamases most closely related to the CTX-M family, sharing $70-75 \%$ amino acid identity (Tzouvelekis et al., 2000; Bonnet, 2004). Analysis of the universal CTX-M-U primers used in this study revealed that the primers used annealed to regions of conserved sequence in both the bla $a_{\mathrm{CTX}-\mathrm{M}}$ and $b l a_{\mathrm{OXY}}$ genes (Figure S3.1). To our knowledge, this is the first report of false positive binding of CTX-M universal primers with OXY-type enzymes.

The $b l a_{\mathrm{OXY}}$ encoded enzyme is a chromosomal class A $\beta$-lactamase discovered and reported in $K$. oxytoca isolates, conferring amino- and carboxy-penicillin resistance (Fevre et al., 2005). K. oxytoca is an opportunistic pathogen and documented causative agent of antibiotic-associated hemorrhagic colitis (Högenauer et al., 2006). Eight $K$. oxytoca were isolated from the Toronto WWTP during this study. Each of the K. oxytoca isolates that were sequenced encoded both KPC-2 and OXY-6-2 enzymes (Figure 3.2). Reports from Brazil and the USA have also characterized clinical $K$. oxytoca isolates producing the K. pneumoniae carbapenemase enzyme KPC-2 (Yigit et al., 2003; Almeida et al., 2013). Shared environments between $K$. pneumoniae and $K$. oxytoca may facilitate horizontal gene transfer and permit adaptation to new environments across species (Moradigaravand et al., 2017). This suggests $K$. oxytoca may be an equally suitable AMR indicator, and is of concern as shared habitats such as wastewater may permit transfer of 
AMR to clinically relevant bacterial species and possibly facilitate entrance of MDR organisms into the environment.

As this study did not include PCR analyses for all possible $\beta$-lactamase-related resistance genes, WGS was conducted for a subset of 46 isolates from each WWTP including isolates with and without PCR-identified resistance genes in order to confirm ARGs, and to investigate additional bla genes that may have contributed to carbapenemtolerance. WGS analyses detected additional $\beta$-lactamase encoding genes including ampS, bla $a_{\mathrm{ACT}}, b l a_{\mathrm{CARB}}, b l a_{\mathrm{CEPH}}, c p h A, b l a_{\mathrm{FOX}}, b l a_{\mathrm{MOX}}, b l a_{\mathrm{OXY}}, b l a_{\mathrm{L} 1}$, and $b l a_{\mathrm{PAM}}$ (Figure 3.2). No ARGs were detected in eleven of the sequenced isolates using ResFinder-2.1 or CARD-RGI (Figure 3.2, isolates: OLC2865, 2866, 2868, 2691, 2871, 3144, 3145, 3147, 3634, 3635, and 3636). ResFinder also failed to detect any ARGs in both Pandoraea apista (Burkholderiaceae) isolates (OLC2679 and 3043), however both had PCR positive results for $b l a_{\mathrm{OXA}-10}$ and CARD-RGI detected $b l a_{\mathrm{OXA}-153}$ at $99.6 \%$ identity (Figure 3.2, Table S3.1). Lowering ResFinder-2.1 thresholds to $80 \%$ ID enabled detection of bla $a_{O X A-62}$ at $83.46 \%$. This suggests the identity thresholds used with ResFinder may have been too stringent, and some ARG-alleles may not be included in the ResFinder database. Similarly, blastn analysis using $b l a_{\mathrm{L} 1}$, a chromosomal metallo- $\beta$-lactamase from Stenotrophomonas maltophilia (Accession: EF126059), resulted in positive hits for the $S$. maltophilia isolates OLC2691, OLC2871, OLC3147, and OLC3635 (Figure 3.2). ResFinder analysis of the S. maltophilia isolates with the \%ID threshold lowered to 80 resulted in positive hits for $b l a_{\mathrm{L} 1}$ at $89.57 \%, 88.69 \%, 89.70 \%$, and $89.70 \%$ respectively. In contrast, CARD-RGI did not detect the bla $a_{\mathrm{L} 1}$ gene in any of these isolates. 
Likewise, ResFinder v2.1 and v3.2 with lower ID threshold reported $b l a_{\mathrm{ACT}-4}$ at $85.08 \%$ and bla $_{\mathrm{ACT}-9}$ at $98.86 \%$, respectively, in the Enterobacter asburiae isolate (OLC3633). Jousset et al. (2019) recently described an Enterobacter kobei lineage with weak carbapenemase activity due to the chromosomally encoded AmpC gene $b l a_{\mathrm{ACT}-28}$. CARD-RGI analysis of OLC3633 detected ACT-28 (accession : NG_048614), which is not present in the ResFinder database, at $98.95 \%$ identity. In contrast to Jousset et al. (2019) who found isolates were resistant or less sensitive to many $\beta$-lactams including ESBLs and expanded spectrum cephalosporins, but not imipenem and meropenem, the Enterobacter asburiae isolated in this study exhibited resistance to meropenem at 4 $\mu \mathrm{g} / \mathrm{mL}$. In addition to AmpCs and ESBLs, porin mutations or modified expression has also been found to confer resistance in Enterobacter species (Codjoe and Donkor, 2017; Hao et al., 2018) and may have contributed to resistance in this isolate.

The remaining seven isolates with no identifiable $\beta$-lactam ARGs were either Pseudomonas fluorescens or Pseudomonas putida (Figure 3.2). Neither CARD-RGI, ResFinder analysis with \%ID and minimum length lowered to minimum values, nor blastn (BLAST with default settings) analyses of sequences to known Pseudomonas spp. metallo- $\beta$-lactamase genes (Thaller et al., 2011; Suzuki et al., 2014; Borgianni et al., 2015) found any identifiable carbapenem-resistance genes in these isolates. It is possible that these Pseudomonas isolates exhibit carbapenem-tolerance due to reduced outer membrane permeability and/or increased efflux as has been described elsewhere (Livermore, 2001, 2002; Tacão et al., 2015; Kittinger et al., 2016; Codjoe and Donkor, 2017; Jousset et al., 2019). 


\subsubsection{Plasmid-encoded ARGs}

MOB-recon predicted a combined 111 plasmids in 24 of the 46 sequenced isolates. Of the 111 plasmids 17 were predicted as conjugative, 34 were predicted as mobilizable, and 24 included contigs which encoded ARGs (Table S3.2). Plasmid incompatibility groups included ColRNAI $(n=5), \operatorname{IncC}(n=2), \operatorname{IncFIA}(n=3)$, IncFIB $(\mathrm{n}=6), \operatorname{IncFII}(\mathrm{n}=7), \operatorname{IncP}(\mathrm{n}=8), \operatorname{IncR}(\mathrm{n}=2), \operatorname{IncQ} 1(\mathrm{n}=2), \operatorname{IncQ} 2(\mathrm{n}=2)$, and $\operatorname{IncX} 3(\mathrm{n}=1)$ (Figure 3.2, Table S3.2).

All five of the sequenced Klebsiella spp. contained plasmids encoding ARGs. In contrast, while each of the six sequenced Aeromonas spp. isolates encoded plasmids, only three of these Aeromonas isolates' plasmids included contigs encoding ARGs (Table S3.2). However, short read sequences such as those generated in this study can be challenging to assemble, preventing complete plasmid sequences from being accurately reconstructed (Orlek et al., 2017). MOB-suite is dependent on a plasmid-database to provide accurate results, and the authors acknowledge that it will not perform well on novel plasmids lacking sequence similarity to those in the database (Robertson and Nash, 2018). The database contents are biased towards plasmids from Enterobacteriaceae (Robertson and Nash, 2018), presenting an additional obstacle. Although some of the resistance genes identified in this study are known to be chromosomal, it is likely that MOB-suite was unable to accurately reconstruct all plasmids for these wastewater isolates as there may not have been matches in current public databases. In order to more accurately determine ARG location in these isolates (Table S3.2), additional work to isolate and transfer plasmid(s) and/or long-read sequencing will need to be conducted. 


\subsubsection{Use of indicator organisms for monitoring AMR in WWTPs}

In addition to monitoring specific ARGs, AMR surveillance programs also recommend monitoring for the presence of bacterial indicators of the Gammaproteobacteria class as these are the most frequent ARG carriers (Berendonk et al., 2015). Pseudomonas aeruginosa and Aeromonas spp. have been proposed as indicators in environmental samples where fecal contamination is not expected. Bacterial indicators where fecal contamination is expected include Escherichia coli, Enterococcus faecalis, Enterococcus faecium, and Klebsiella spp. The recommended indicator organisms are ubiquitous as well as carriers of clinically relevant ARG(s) in multiple environments. A variety of different bacterial species were identified using MALDI-TOF (Figure 3.1b) and WGS (Figure 3.2). Suggested AMR-indicators of the genera Aeromonas and Klebsiella were isolated from both the Toronto and Ottawa samples tested (Figure 3.1b, Table S3.1), which correlated with an increased number of carbapenem-resistance genes (Figure 3.1a). Every Klebsiella and Aeromonas spp. isolated and identified during this study harboured multiple ARGs, some of which were located on plasmids.

Klebsiella spp. have been recommended as possible indicators to assess AMR status in environmental settings as they are considered early indicators of new AMR emergence (Berendonk et al., 2015; Navon-Venezia et al., 2017). We identified eight $K$. oxytoca and one $K$. pneumoniae in the Toronto and Ottawa samples (respectively) (Sievert et al., 2013; Moradigaravand et al., 2017). The K. pneumoniae isolated from Ottawa was notable for its MDR genotype. It was predicted to contain several plasmids and multiple ARGs including resistance to aminoglycosides, fluoroquinolones, 
macrolides, phenicols, sulphonamides, and trimethoprim, in addition to the $\beta$-lactamases bla $_{\mathrm{TEM}-1}$, bla $_{\mathrm{SHV}-11}, b l a_{\mathrm{KPC}-3}, b l a_{\mathrm{CARB}-2}$, and bla $_{\mathrm{OXA}-9}(\mathrm{OLC} 2685$, Figure 3.2$)$. Netikul and Kiratisin (Netikul and Kiratisin, 2015) found carbapenem-resistant K. pneumoniae isolated from a university hospital in Thailand were resistant to carbapenems at a higher ratio than other CREs, harboured multiple $\beta$-lactamase and ESBL genes, and/or expressed altered porin expression, yet only 2 isolates (out of 36 they tested) encoded commonly associated carbapenemase genes (eg. bla $\left.a_{\mathrm{KPC}}\right)$, suggesting the need to investigate other mechanisms of carbapenem-resistance in isolates.

Other than Klebsiella spp. a number of isolates belonging to the AMR-indicator genus Aeromonas were also isolated from the Toronto and Ottawa sites (Figure 3.1b, Figure 3.2), although this genus is suggested as an indicator where fecal contamination is not expected. Members of the genus Aeromonas are ubiquitous opportunistic pathogens primarily found in aquatic environments but have also been isolated from clinical, soil, and animal environments (Varela et al., 2016; Chenia and Duma, 2017). Several studies have investigated members of Aeromonas as a reservoir of AMR in aquatic environments (Figueira et al., 2011; Trudel et al., 2016; Varela et al., 2016). Trudel et al. (2016) found $37 \%$ of tested Canadian $A$. salmonicida isolates contained at least one resistance plasmid and could pose a potential risk for transfer of resistant genetic material to other waterborne bacteria (including Salmonella spp.). MALDI-TOF identified 3 isolates from each of the Toronto and Ottawa samples as Aeromonas spp. (Table S3.1). WGS analysis identified six Aeromonas isolates from Toronto $(\mathrm{n}=4)$ and Ottawa $(\mathrm{n}=2)$, four and three of which encoded $b l a_{\mathrm{GES}}$ and $b l a_{\mathrm{OXA}} \beta$-lactamase genes, respectively. WGS detected $\beta$ lactamase ARGs bla $a_{\mathrm{FOX}}$, ampS, bla $\mathrm{OXX}_{\mathrm{XA}}, b l a_{\mathrm{GES}}, b l a_{C E P H}, c p h A 2$, and $b l a_{\mathrm{MOX}-6}$ in 
aeromonads isolated during this study (Figure 3.2). Overall, 10 Aeromonas spp. isolates were identified using a combination of MALDI-TOF and WGS from Toronto $(n=5)$ and Ottawa (n=5) (Table S3.1).

Species identification of Arnprior isolates, which had few known carbapenem or $\beta$-lactam resistance genes, found a large proportion of pseudomonads that were not $P$. aeruginosa (Figure 3.1, Figure 3.2). AMR in P. aeruginosa has been attributed to expression of modifying enzymes, efflux pumps, lower membrane permeability, and chromosomal mutations (Moriyama et al., 2009; Chatterjee, 2016). While $P$. aeruginosa has been well studied and is documented to be inherently resistant and exhibit decreased susceptibility to many antibiotics (Livermore, 2002; Breidenstein et al., 2011) there are relatively few studies regarding AMR mechanisms in $P$. fluorescens and $P$. putida. It is possible that carbapenem resistance in the pseudomonads isolated from Arnprior is due to presence of chromosomal $\operatorname{amp} C$ genes and/or altered membrane permeability as has been observed in other Pseudomonas species (Breidenstein et al., 2011; Molina et al., 2014; Suzuki et al., 2014; Borgianni et al., 2015).

\subsection{Conclusion}

Wastewater is an important source of AMR where there is potential for widespread dissemination into the environment. As clinical, industrial, household, and agricultural practices all connect to wastewater systems, this also makes WWTPs useful sites to monitor the AMR burden within an environment and assess the risk of transfer to humans both directly and indirectly through food production. A large number of indicator genera (e.g. Klebsiella) and organisms harbouring AMR indicator resistance genes (e.g. 
bla $a_{\mathrm{KPC}}, b l a_{\mathrm{TEM}}, b l a_{\mathrm{VIM}}$, etc) were identified in both Toronto and Ottawa influent samples. Only a single Pandoraea sp. isolate purified from the smaller rural community of Arnprior harboured a $\beta$-lactamase resistance gene (blaXA-153), which has been reported to cause elevated MICs to carbapenems (Schneider and Bauernfeind, 2015). None of the isolates from Arnprior were identified as AMR indicator species.

Limitations of this study include the small sample size at each WWTP site; PCR analyses not being conducted for all known carbapenem-inactivating ARGs; that other $\beta$ lactamase enzymes may also hydrolyze carbapenems to some extent resulting carbapenem-tolerance; that efflux and porin mutations possibly conferring carbapenemtolerance were not investigated; and that the selection method used was not comprehensive for all bacteria found in wastewater. Future studies would ideally sample a number of times over multiple seasons, as well as test a larger number of isolates and account for clones within samples. While this study utilized PCR and WGS of bacteria isolated from samples, other molecular techniques including quantitative PCR (qPCR), metagenomics, and 16S rRNA sequencing allow rapid sample analysis while avoiding cultivation and have previously been used to study wastewater (Böckelmann et al., 2009; Muurinen et al., 2017; Ng et al., 2017; Freeman et al., 2018; Rocha et al., 2019).

We found that although PCR is an economical high-throughput method for gene detection, when phenotypes could be the product of a multitude of genes including carbapenemases, cephalosporinases, and ESBLs, requiring multiple experiments and multiplex-PCRs, WGS may actually be more beneficial. These results highlight the value of WGS for AMR detection in bacterial isolates, especially for genera that are not routinely monitored. However, we did find both ResFinder-2.1 and CARD-RGI to be 
biased towards well studied bacteria, such as members of Enterobacteriaceae, and suggest investigations of non-Enterobacteriaceae may benefit from using lower geneidentity thresholds or conducting analyses with a more comprehensive AMR-gene database. The inability to determine the cause of carbapenem-tolerance in seven Pseudomonas spp. isolates is evidence additional, uncharacterized resistance mechanisms still exist. 


\section{Chapter 4: Genomic Markers for Quaternary Ammonium Compound \\ Resistance as a Persistence Indicator for Listeria monocytogenes \\ Contamination in Food Manufacturing Environments}

This chapter is an adaptation of the following publication:

Cooper, A.L. and Carrillo, C.D., Deschênes, M. \& Blais, B. (2020). Quaternary ammonium compound resistance as a persistence marker for L. monocytogenes. Journal of Food Protection. 


\subsection{Abstract}

Persistent contamination of food manufacturing environments by Listeria monocytogenes is an important public health risk because such contamination events defy standard sanitization protocols, for example, the application of quaternary ammonium compounds (QACs) such as benzalkonium chloride (BC), providing a source for prolonged dissemination of the bacteria in food products. We performed whole-genome sequence (WGS) analyses of 1279 well-characterized L. monocytogenes isolates from a variety of foods and food manufacturing environments and identified the $b c r A B C$ gene cassette associated with $\mathrm{BC}$ resistance in $531(41.5 \%)$ isolates. The $b c r A B C$ cassette was significantly associated with L. monocytogenes isolates belonging to clonal complexes (CC) 321, 155, 204 and 199, which are among the ten most prevalent genotypes recovered from foods and food-production environments. Notably, all but one of the 177 CC321 isolates harbored the $b c r A B C$ cassette. Three-hundred and eighty-four $(38.6 \%)$ of the 994 isolates recovered from foods representing 67 different CCs, and 119 (59.2\%) of isolates from food-manufacturing environmental samples representing 26 different CCs, were found to harbor the intact $b c r A B C$ cassette. A representative set of 69 isolates with and without $b c r A B C$ was assayed for the ability to grow in the presence of $\mathrm{BC}$, and 34 of 35 isolates harboring the $b c r A B C$ cassette exhibited minimum inhibitory concentrations of $\geq 10 \mu \mathrm{g} / \mathrm{mL}$ BC. Determination of $b c r A B C$ in isolates could be achieved using both polymerase chain reaction and WGS techniques, providing food testing laboratories with options for the characterization of isolates. The ability to determine QAC-resistance markers such as $b c r A B C$ and epidemiologic lineage may ultimately provide risk managers with a tool to assess the potential for persistent contamination of the food 
manufacturing environment and the need for more targeted surveillance to ensure the efficacy of mitigation actions.

\subsection{Introduction}

The presence of pathogens such as Listeria monocytogenes in food manufacturing environments is a major source of contamination for food products which are the primary source of listeriosis outbreaks (Gombas et al., 2003; Gaulin et al., 2012; Cartwright et al., 2013; Currie et al., 2015; Garner and Kathariou, 2016). Of particular concern is the phenomenon of persistent contamination (Lundén et al., 2002; Gaulin et al., 2012; Ferreira et al., 2014; Leong et al., 2014; Martínez-Suárez et al., 2016; Ortiz et al., 2016), where a strain remains firmly established in the environment, resisting standard sanitization protocols such as the application of quaternary ammonium compounds (e.g., benzalkonium chloride). In 2008 Canada experienced the worst listeriosis outbreak in its history, with 22 lives lost and an estimated 57 serious illnesses due to contamination of meat slicing equipment by L. monocytogenes defying all attempts to decontaminate the plant after it had been repeatedly identified in the environment (Government of Canada, 2009a; Currie et al., 2015). Following genomic sequence analyses, a novel mobile genetic element encoding the multi-drug resistant efflux pump encoded by emrE was postulated to be associated with persistence of outbreak-related strains (Gilmour et al., 2010; Bergholz et al., 2018).

While the causes for L. monocytogenes persistence may vary, a link to benzalkonium chloride (BC) resistance has been suggested (Elhanafi et al., 2010; Ferreira et al., 2014; Martínez-Suárez et al., 2016; Ortiz et al., 2016; Møretrø et al., 2017; Cherifi 
et al., 2018; Minarovičová et al., 2018). An important determinant of BC resistance is the efflux system encoded by the bcrABC gene cassette (Elhanafi et al., 2010). Studies have revealed that the majority of $L$. monocytogenes strains resistant to $\mathrm{BC}$ carried the $b c r A B C$ cassette (Dutta et al., 2013), and that the cassette is mobile and may be transferrable to other bacteria, such as other Listeria species (Katharios-Lanwermeyer et al., 2012) and Escherichia coli (Xu et al., 2016). In a recent study characterizing the genomes of $L$. monocytogenes isolates collected at a pig slaughterhouse we found that successive environmental isolates (persistent types) linked on the basis of single nucleotide variant (SNV) analysis all harbored $b c r A B C$, and concluded that both high resolution typing and determination of the cassette may serve to distinguish between persistent and sporadic $L$. monocytogenes isolates (Cherifi et al., 2018). This in turn may have important ramifications for risk management actions when L. monocytogenes is recovered from a food manufacturing environment, since the ability of a strain to persist casts doubt on the efficacy of standard sanitization protocols, and more intensive cleaning procedures (e.g., equipment teardown, use of alternative sanitizers) may be warranted.

During the past four-year period, our laboratory has undertaken a program of whole genome sequencing (WGS) and analysis of the Canadian Food Inspection Agency (CFIA) culture collection. Bacterial isolates were assembled through surveillance and regulatory food inspection activities, including 1,463 historical L. monocytogenes isolates recovered from various food samples and their manufacturing environments. We have developed bioinformatics processes to analyze WGS data from food-borne bacterial isolates in near real-time for the purposes of establishing identity, determining specific gene sequences and high resolution SNV typing (Lambert et al., 2015, 2017; Carrillo et 
al., 2016, 2019) to inform regulatory actions. We sought to take advantage of these resources by analyzing the CFIA $L$. monocytogenes collection to determine the distribution of $b c r A B C$ and other sanitizer resistance genes known to occur in $L$. monocytogenes, such as qacC (Paulsen et al., 1995; Poole, 2005; Pirone-Davies et al., 2018), qacH (Poole, 2005; Ortiz et al., 2016) and emrE (Kovacevic et al., 2016), according to sub-types and provenance, and to ascertain the relationship between the occurrence of the cassette and benzalkonium resistance. The ultimate goal was to determine the utility of $b c r A B C$ as a marker for persistence, consider its possible role in guiding food safety interventions, and suggest convenient analytical tools (e.g., polymerase chain reaction or WGS) for use by food testing laboratories in its determination.

\subsection{Materials and Methods}

\subsubsection{Bacterial isolates}

L. monocytogenes isolates from the Ottawa Laboratory Carling (Canadian Food Inspection Agency) culture collection were routinely grown on Brain Heart Infusion (BHI) agar (Oxoid Ltd., England) for $18-24 \mathrm{~h}$ at $30^{\circ} \mathrm{C}$. Bacterial broth culture suspensions (below) were counted by plating serial dilutions on BHI plates which were incubated for $18-24 \mathrm{~h}$ at $30^{\circ} \mathrm{C}$. 


\subsubsection{Whole-genome sequencing.}

Genomic DNA was extracted for sequencing as follows, BHI broth (Oxoid) was inoculated with a single bacterial colony and incubated for 4 to $6 \mathrm{~h}$ at $36^{\circ} \mathrm{C}$. To collect bacterial cells $400 \mu \mathrm{L}$ of culture was centrifuged at 11,000 rpm for $2 \mathrm{~min}$., the pellet was resuspended in $200 \mu \mathrm{L}$ of lysis buffer containing 1\% Triton X-100 (Sigma-Aldrich, ON, Canada), and $10 \mathrm{mg} / \mathrm{mL}$ lysozyme (Fisher scientific, ON, Canada) and incubated at $37^{\circ} \mathrm{C}$ for $20 \mathrm{~min}$. at $800 \mathrm{rpm}$ in an Eppendorf Thermomixer C (Fisher scientific). Genomic DNA was isolated from lysates using the Maxwell ${ }^{\circledR} 16$ Cell SEV DNA Purification kit (Promega, Madison, WI). DNA was then quantified using the Quant-iT ${ }^{\mathrm{TM}}$ HighSensitivity DNA Assay Kit (Life Technologies Inc., Burlington, ON). Sequencing libraries were constructed from 1 ng of gDNA using Nextera XT DNA Sample Preparation and Index Kits (Illumina, Inc., San Diego, CA) according to manufacturers' instructions. Paired-end sequencing was performed on the Illumina MiSeq platform using 600 cycle MiSeq reagent kits (v3) with 6\% PhiX control (Illumina).

\subsubsection{Sequence analysis}

Quality of raw sequencing reads was assessed with FastQC version 0.11.8 (Andrews, 2010), quality trimmed with BBDuk from BBTools version 38.22 (Bushnell, 2014) with the following parameters: trim quality of 10 and removal of reads below 50 bp long. Error correction was performed using tadpole version 8.22 from BBTools (Bushnell, 2014) in 'correct' mode with default parameters. Contigs were assembled from the trimmed and error-corrected reads using SKESA version 2.3.0 with the vector percent argument disabled (Souvorov et al., 2018). Assembly quality was assessed with 
qualimap version 2.2 (García-Alcalde et al., 2012; Okonechnikov et al., 2016), and quast version 2.3 (Gurevich et al., 2013). Sequences were checked for contamination using ConFindr 0.5.0 with default settings (Low et al., 2019). Isolate characterization was conducted using custom Python scripts (https://github.com/OLC-

Bioinformatics/COWBAT/tree/v0.1.5). Isolates were determined to be L. monocytogenes based on detection of key indicator genes $h l y A$ and inlJ (Blais et al., 2015). Genotyping was conducted on sequence assemblies using the Institut Pasteur BIGSdb-Lm platform for Mulilocus sequence typing (MLST), core genome MLST (cgMLST) and to determine clonal complexes and lineages (available at: https://bigsdb.pasteur.fr/listeria/) (Moura et al., 2016) and Ribosomal MLST (rMLST) profiles (Jolley et al., 2012) were determined using databases available at https://pubmlst.org/rmlst/ (Jolley et al., 2012). Clonal complexes are groups of allelic profiles sharing at least six of seven genes with other

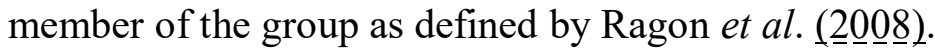

SNV typing was conducted using the Single Nucleotide Variant PHYLogenomics (SNVPhyl) pipeline version 1.0.1 (Petkau et al., 2017). High-quality SNVs had a minimum coverage of 5 reads, with $75 \%$ of reads supporting the SNV identification, and a filter density window of 500 with a density threshold of 2.

Plasmid(s) were predicted and reconstructed from assembled genomes using the MOB-recon tool from MOB-suite v 1.4.1 (Robertson and Nash, 2018).

\subsubsection{Antimicrobial and biocide resistance gene detection in silico}

The NCBI AMRFinderPlus Reference Gene Catalog and AMR_CDS database version 2019-20-30.1 were downloaded from the NCBI FTP server on 2019-11-01 
(ftp://ftp.ncbi.nlm.nih.gov/pathogen/Antimicrobial_resistance/AMRFinderPlus/database/ $3.2 / 2019-10-30.1 /$ ). Using the reference gene catalog as a guide, the coding sequences in the AMR_CDS database were divided into antimicrobial resistance, metal resistance, quaternary ammonium compound (QAC) resistance, and efflux resistance gene databases. Virulence genes, AMR point mutation genes, and $b l a_{\mathrm{EC}}$ alleles from the NCBI AMRFinderPlus database were not included. Additional gene sequences added to the resistance database included L. monocytogenes genes: $b c r A, b c r B$, and $b c r C$ accession JX023276.1; emrE, accession CP007020.1; Lde, accession NZ_LR134400.1; $m d r L$, accession NZ_LR134403.1; qacA, accession KC980922; qacC, accession RJZ34303; qacED1, accession KM972592.1; and qacH, accession HG329628.1. These gene databases were then used with the $k$-mer alignment (KMA) tool version 1.17 (Clausen $e t$ al., 2018) to analyze all WGS assemblies for resistance genes.

\subsubsection{Identification and removal of duplicate isolates}

Metadata and WGS data for each of the 1,463 isolates was used to identify possible L. monocytogenes clones. Isolates recovered from the same sample were flagged, and those which had matching cgMLST and resistance profiles were considered the same strain. A single representative isolate sequence was selected for each sample. After removal of duplicates, 1,297 L. monocytogenes WGS datasets were retained for analysis (Table S4.1). 


\subsubsection{Nucleotide sequence accession numbers}

Raw data have been deposited at DDBJ/EMBL/GenBank under BioProject PRJNA435747. The accession numbers, isolate descriptions, and investigated resistance genes are listed in Table S4.1.

\subsubsection{Multiplex $b c r A, b c r B$ and $b c r C$ Polymerase chain reaction (PCR)}

Bacterial lysates were prepared by suspension of a colony in $100 \mu 1$ of $1 \%(\mathrm{v} / \mathrm{v})$ Triton X-100 (Sigma-Aldrich, Canada), followed by heating at $80^{\circ} \mathrm{C}$ for $5 \mathrm{~min}$, and $5 \mu \mathrm{L}$ of lysate was combined with of $45 \mu \mathrm{L}$ of reaction mixture containing 2.5 units HotStar Taq, 1.0 X HotStar PCR buffer (giving a final $\mathrm{MgCl}_{2}$ concentration of $1.5 \mathrm{mM}$ ) (Qiagen Inc., Canada), $200 \mu \mathrm{M}$ of each $\mathrm{dNTP}, 0.2 \mu \mathrm{M}$ of each of the forward and reverse primers previously described for $b c r A, B$ and $C$ (Minarovičová et al., 2018). The PCR was carried out in a Mastercycler gradient thermal cycler (Eppendorf, Mississauga, Ontario, Canada) using the following conditions: an initial denaturation cycle at $94^{\circ} \mathrm{C}$ for $15 \mathrm{~min}$, followed by 35 cycles of denaturation at $94^{\circ} \mathrm{C}$ for 30 seconds, primer annealing at $55^{\circ} \mathrm{C}$ for 30 seconds, and elongation at $72^{\circ} \mathrm{C}$ for $30 \mathrm{~s}$, with an additional $2 \mathrm{~min}$ at $72^{\circ} \mathrm{C}$ following the last cycle. Amplicons were analyzed by capillary electrophoresis using the QIAxcel (Qiagen) DNA screening system with method AM320 and alignment markers 15 to 3000 bp (following the manufacturer's instructions).

\subsubsection{Benzalkonium chloride (BC) susceptibility testing}

Strains were grown in brain heart infusion broth (BHI) (Oxoid) for 18-24h at $30^{\circ} \mathrm{C}$. Culture tubes of $3 \mathrm{~mL}$ BHI containing 0 to $14 \mu \mathrm{g} / \mathrm{mL}$ of benzalkonium chloride 
(BC) (Acros Organics, USA) were inoculated with approximately 300 CFU of broth culture for each subject strain. Inoculated tubes containing BC were incubated for $48 \mathrm{~h}$ at $30^{\circ} \mathrm{C}$, and the optical density (OD, $\left.600 \mathrm{~nm}\right)$ of the cultures was measured using a portable cell density meter (Cell Density Meter Model 40; Fisher scientific, Canada). The ability of a strain to grow in sanitizer was determined turbidimetrically where an $\mathrm{OD}_{600}$ of $>0.2$ was considered as positive visible growth. For each isolate the minimum inhibitory concentration (MIC) was defined as the lowest concentration of BC to completely inhibit growth, which equated to an $\mathrm{OD}_{600}$ of 0.05 or less.

\subsubsection{Statistical tests to identify associations of QAC-resistance with food sources and CCs}

To estimate the significance of QAC-resistance gene carriage associated with different food sources and CCs. Contingency tables were created to identify relationships between QAC-R gene carriage in isolates from animal product sources (dairy, fish/seafood, meat, eggs, and their production environments) or fruit/vegetable sources and the proportion of QAC-R gene carriage in the entire dataset (1279 isolates). Likewise, QAC-R gene carriage within each of the ten most commonly isolated CCs (5, $321,8,9,155,7,204,11,199$, and 14) was compared to carriage within the entire dataset. Chi-square tests were performed using R statistical software version 4.0.0 (R Core Team, 2014). Multiple tests were accounted for using the sequential Bonferroni correction as described by Maury et al. (2016). 


\subsection{Results and Discussion}

\subsubsection{WGS analyses of $L$. monocytogenes strains}

A total of 1463 L. monocytogenes isolates from the CFIA culture collection, including strains collected between 2000 and 2019 were subjected to WGS analysis for determination of sequence types (STs), clonal complexes (CCs), and carriage of antimicrobial-, and QAC-resistance genes (Table S4.1). Removal of probable clones isolated from the same sample left 1279 isolates for QAC-resistance investigations. Of these, 994 isolates representing 67 different CCs were from ready-to-eat (RTE) foods including meat, dairy, fish/seafood, fruits and vegetables, with 384 (38.6\%) having bcr $A B C$. A total of 201 isolates representing $25 \mathrm{CCs}$ were recovered from meat, dairy, fruit/vegetable, or fish/seafood production environments, with 119 (59.2\%) having bcr $A B C$. The most commonly occurring CCs in foods and environmental samples were CC5, CC321, CC8, CC9, and CC155, representing 52.2\% of the total number of isolates examined in this study (Table 4.1, Figure 4.1). Interestingly, 99.5\% (176 of 177) of strains belonging to CC321, one of the most commonly observed clonal complexes, harbored the bcrABC cassette (Figure 4.1). All 1279 of the L. monocytogenes isolates analyzed in this study encoded $L d e$ and $m d r L$, whereas none harboured qacA or qacEDl (Table S4.1). In addition, genomic analysis revealed that emrE, qacH, and qacC occurred in 5.94, 1.09 and $1.72 \%$ of all strains, respectively, making $b c r A B C$ by far the most common genetic element conferring resistance to $\mathrm{BC}$. 
Table 4.1. Listeria monocytogenes bcr $A B C$ profiles by clonal complex and source ${ }^{\mathrm{a}}$

\begin{tabular}{|c|c|c|c|c|c|}
\hline $\begin{array}{l}\text { Clonal } \\
\text { Complex } \\
\end{array}$ & Egg & Dairy & Fish/Seafood & Fruit/Vegetables & $\begin{array}{l}\text { RTE: } \\
\text { Meat }\end{array}$ \\
\hline $\mathrm{CC} 1$ & - & $14(0)$ & $5(40)$ & $5(0)$ & $5(0)$ \\
\hline $\mathrm{CC} 2$ & - & $1(0)$ & $3(33.33)$ & $2(0)$ & $6(0)$ \\
\hline $\mathrm{CC} 3$ & - & $3(0)$ & $4(0)$ & $2(100)$ & $7(28.57)$ \\
\hline CC5 & - & $34(70.59)$ & $21(14.29)$ & $13(30.77)$ & $55(69.09)$ \\
\hline CC6 & - & $2(0)$ & $6(0)$ & $4(25)$ & $9(0)$ \\
\hline $\mathrm{CC} 7$ & $4(0)$ & $4(0)$ & $7(14.29)$ & $17(17.65)$ & $25(4)$ \\
\hline $\mathrm{CC} 8$ & - & $6(0)$ & $15(6.67)$ & $2(0)$ & $64(0)$ \\
\hline CC9 & - & - & $12(83.33)$ & $6(33.33)$ & $64(23.44)$ \\
\hline $\mathrm{CC} 11$ & - & $2(0)$ & - & $16(18.75)$ & $14(7.14)$ \\
\hline $\mathrm{CC} 14$ & $2(0)$ & $3(0)$ & $2(0)$ & $19(0)$ & $6(16.67)$ \\
\hline $\mathrm{CC} 20$ & - & - & $1(0)$ & - & $1(100)$ \\
\hline $\mathrm{CC} 26$ & $1(0)$ & - & - & $1(100)$ & - \\
\hline $\mathrm{CC} 31$ & - & $11(90.91)$ & $2(50)$ & $1(0)$ & $1(0)$ \\
\hline CC88 & - & - & $3(100)$ & - & - \\
\hline $\mathrm{CC} 155$ & - & $31(100)$ & $16(62.5)$ & $3(66.67)$ & $17(76.47)$ \\
\hline CC193 & - & - & $3(0)$ & - & $1(100)$ \\
\hline CC199 & - & $3(100)$ & $1(100)$ & $7(85.71)$ & $12(91.67)$ \\
\hline CC204 & - & $1(0)$ & - & $18(100)$ & $32(75)$ \\
\hline CC321 & - & $2(100)$ & $44(97.73)$ & $9(100)$ & $76(100)$ \\
\hline $\mathrm{CC} 415$ & - & $2(0)$ & $3(100)$ & $1(0)$ & - \\
\hline
\end{tabular}

Abbreviations: CC, clonal complex; RTE, ready-to-eat.

${ }^{\mathrm{a}}$ Listeria monocytogenes isolates recovered from a variety of samples at the Canadian Food Inspection Agency were analyzed by whole genome sequencing to determine clonal complexes $(\mathrm{CC})$ and the presence of the $b c r A B C$ gene cassette. Numbers under each source category indicate the total number of isolates of corresponding $\mathrm{CC}$ from that source, with the percentage harbouring $b c r A B C$ indicated in parentheses. Note that only CCs with a $b c r A B C$ cassette in at least one isolate were included in this table. 


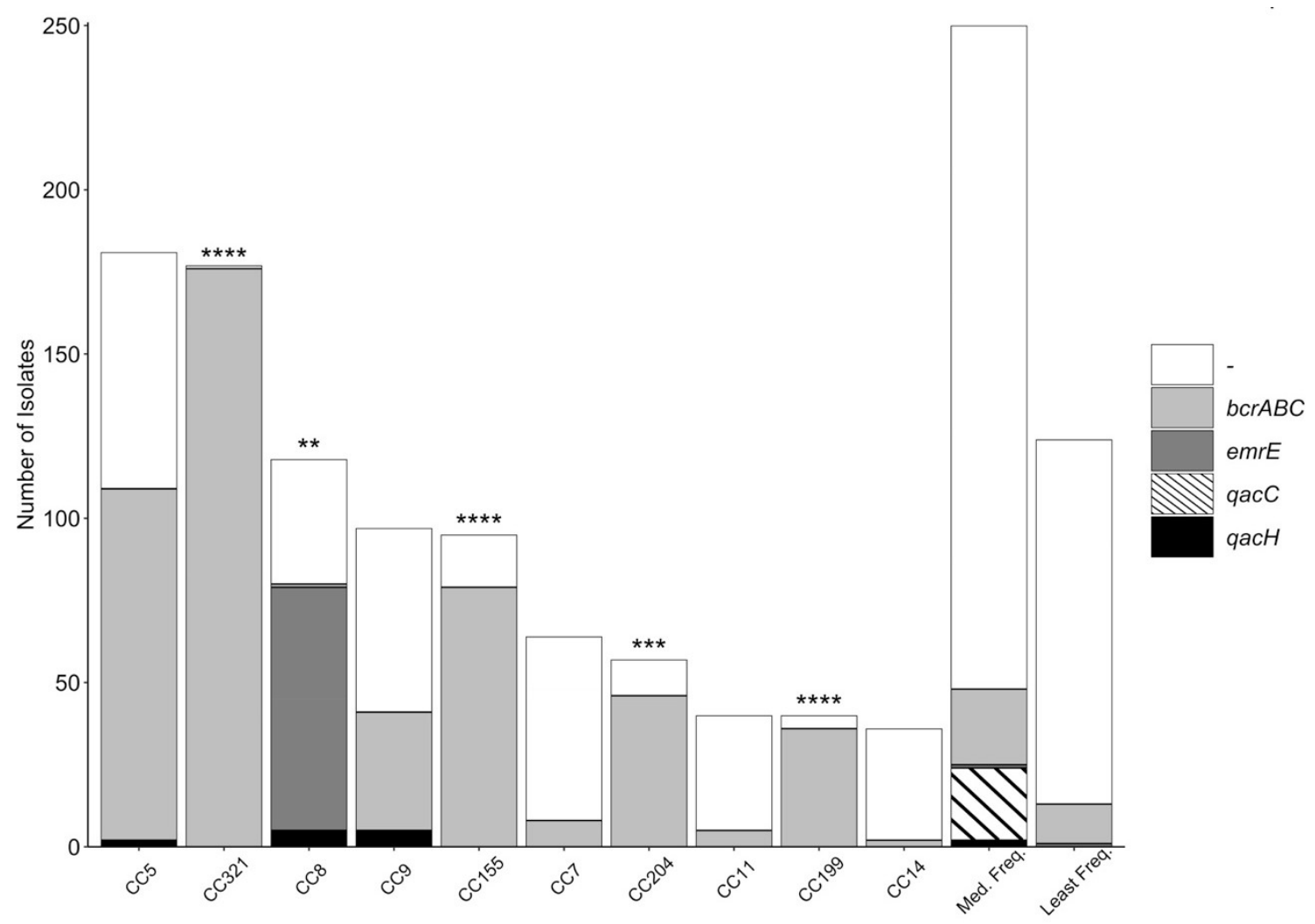

Figure 4.1. Quaternary ammonium compound (QAC) resistance genes are common in Listeria monocytogenes isolates recovered from food samples. A set of $1279 \mathrm{~L}$. monocytogenes isolated from food and food-manufacturing environments were sequenced and analysed for carriage of common QAC-resistance genes. Frequency of QAC gene carriage (y-axis) (no QAC gene denoted by “_-") is plotted for the ten most commonly isolated clonal complexes (CC), ranked by number of isolates in each $\mathrm{CC}$ (xaxis). CCs containing 10 to 35 isolates (Med. Freq.), and CCs containing less than 10 isolates (Least Freq.) were grouped (x-axis). Significance of association between CC and QAC-resistance was conducted for the ten most commonly isolated CCs using Chisquared with Bonferroni correction. $\mathrm{p}<0.05=* ; \mathrm{p}<0.01=* * ; \mathrm{p}<0.001=* * * ; \mathrm{p}<$ $0.0001=* * * *$. Significance of association of CCs to be QAC-sensitive is not shown. 
Associations of QAC-resistance gene carriage were not observed for isolates from dairy nor fish/seafood sources (corrected $\mathrm{p}$-values $=1$ ). Significant relationships were observed for QAC-resistance gene carriage in both meat and fruit/vegetable sources, where isolates from meat were slightly more likely to exhibit QAC-resistance (corrected $\mathrm{p}$-value $=0.013)$ in contrast to fruit/vegetables where isolates were more strongly associated with an absence of QAC-resistance genes (corrected p-value $<0.0000$ ). When grouping animal product sources, statistical analysis found a significant association of QAC-resistance gene carriage in isolates from animal products and production environments (556 of 962 , corrected $p$-value $=0.00096)$, contrasting with fruit $/$ vegetable source isolates which were significantly less likely to carry QAC-resistance genes (186 of 241 , corrected p-value $<0.00000$ ) compared to carriage in all 1279 isolates. The observation that QAC-resistant L. monocytogenes are more highly associated with isolates recovered from meat production environments relative to fruit and vegetable production warrants further investigation. Persistence of QAC-resistant L. monocytogenes in meat and animal product manufacturing environments has been reported in several recent studies (Cherifi et al., 2018; Martínez-Suárez et al., 2016; Ortiz et al., 2016). Differences in production practices in fruit and vegetable manufacturing environments may be responsible for lower rates of QAC-resistance in these environments. There were also marked differences in the proportions of QAC-resistance gene carriage among clonal complexes, where resistance gene carriage was strongly associated with some of the most commonly isolated CCs (Figure 4.1) and the absence of QAC resistance genes was strongly associated with others (data not shown). 
It is tempting to speculate that $b c r A B C$ carriage is a significant contributor to persistence in food manufacturing environments since CC321 is a commonly isolated sequence type which is almost always associated with the resistance cassette (Figure 4.1). Naditz et al. (2019) found plasmids harbouring stress response genes significantly contributed to survival of L. monocytogenes in food processing environment conditions. Nonetheless, the occurrence of $b c r A B C$ even in sporadic food or environmental contaminants may not always result in persistence because other factors and conditions, such as biofilm and mechanical entrapment, may also contribute to its manifestation (Møretrø and Langsrud, 2004). A review by Carpentier and Cerf (2011) strongly suggested that L. monocytogenes persistence in food production results from conditions promoting bacterial growth such as harborage sites in equipment, and temperature. The authors mention a number of laboratory studies where persistent $L$. monocytogenes strains were not found to be more resistant or tolerant to disinfectants such as quaternary ammonium compounds. However, these studies all tested a small number of isolates recovered from food production sources in vitro to determine whether they exhibited increased resistance to sanitizers, in contrast to this study which investigated the occurrence of resistance-conferring genes in a large number of strains isolated from food sources over multiple years.

\subsubsection{Correlation of expressed resistance with occurrence of the $b c r A B C$ cassette}

A sub-sample of the L. monocytogenes isolates representing different food and environmental sources and clonal complexes was assayed for the $\mathrm{BC}$ resistance phenotype. The genetic sanitizer resistance profiles, isolation source and STs of the $69 \mathrm{~L}$. 
monocytogenes strains examined are presented in Appendix D.1 - Table 4.2. All but one (CFIAFB20100068) of the 35 isolates harboring the $b c r A B C$ cassette were resistant to BC concentrations of $\geq 10 \mu \mathrm{g} / \mathrm{mL}$. Whereas CFIAFB20100068 exhibited an MIC of 6 $\mu \mathrm{g} / \mathrm{mL}$ BC, MICs of 10-14 $\mu \mathrm{g} / \mathrm{mL}$ BC in BHI were observed for most isolates encoding $b c r A B C$ (Figure 4.2). It is possible that the $b c r A B C$ cassette was not expressed in this isolate, or that the plasmid was lost during passaging of the isolate in preparation for the test. Previous investigators have also found MICs to be variable among $b c r A B C$-carrying isolates (Harrand et al., 2020), though because of the expedient broth-based approach used presently the precise MIC values may differ.

Three exceptions were found where strains lacking the cassette exhibited growth in $\mathrm{BC}$ concentrations of 6-8 $\mu \mathrm{g} / \mathrm{mL}$ (Figure 4.2). In addition to $b c r A B C$, other genetic determinants have been found to enhance tolerance to QACs including emrE (Kovacevic et al., 2016), qacC (Paulsen et al., 1995; Poole, 2005; Pirone-Davies et al., 2018), and qacH (Poole, 2005; Ortiz et al., 2016). Two of the $b c r A B C$-negative exceptions were found to carry the alternative QAC resistance determinants qacC (CFIAFB20110012) and emrE (CFIAFB20090092) (Appendix D.1 - Table 4.2). The remaining exception, CFIAFB20140002, contained no currently identifiable QAC-resistance genes. Previous investigations of the chromosomal efflux pump MdrL (multidrug-resistant Listeria) demonstrated that for strains without plasmid-encoded efflux pumps expression of chromosomal efflux pumps was induced by BC (Romanova et al., 2002; Jiang et al., 2016). Similarly, increased transcription of the multidrug resistance (MDR) transporter Lde (Listeria drug efflux) (Rakic-Martinez et al., 2011; Kovacevic et al., 2016; Ortiz et al., 2016; Meier et al., 2017; Haubert et al., 2019), and cell wall modifications leading to 
a reduction in permeability have been implicated in $\mathrm{BC}$ tolerance in L. monocytogenes (discussed by Meier et al. (2017). Collectively, this suggests the possibility of altered cell permeability and/or increased transcription of $M d r L$ or $L d e$ producing $\mathrm{BC}$ resistance in CFIAFB20140002, which should be investigated further. Of the 1279 L. monocytogenes isolates in this study $531,76,14$, and 22 isolates encoded $b c r A B C, e m r E$, qacH, and $q a c C$, respectively.

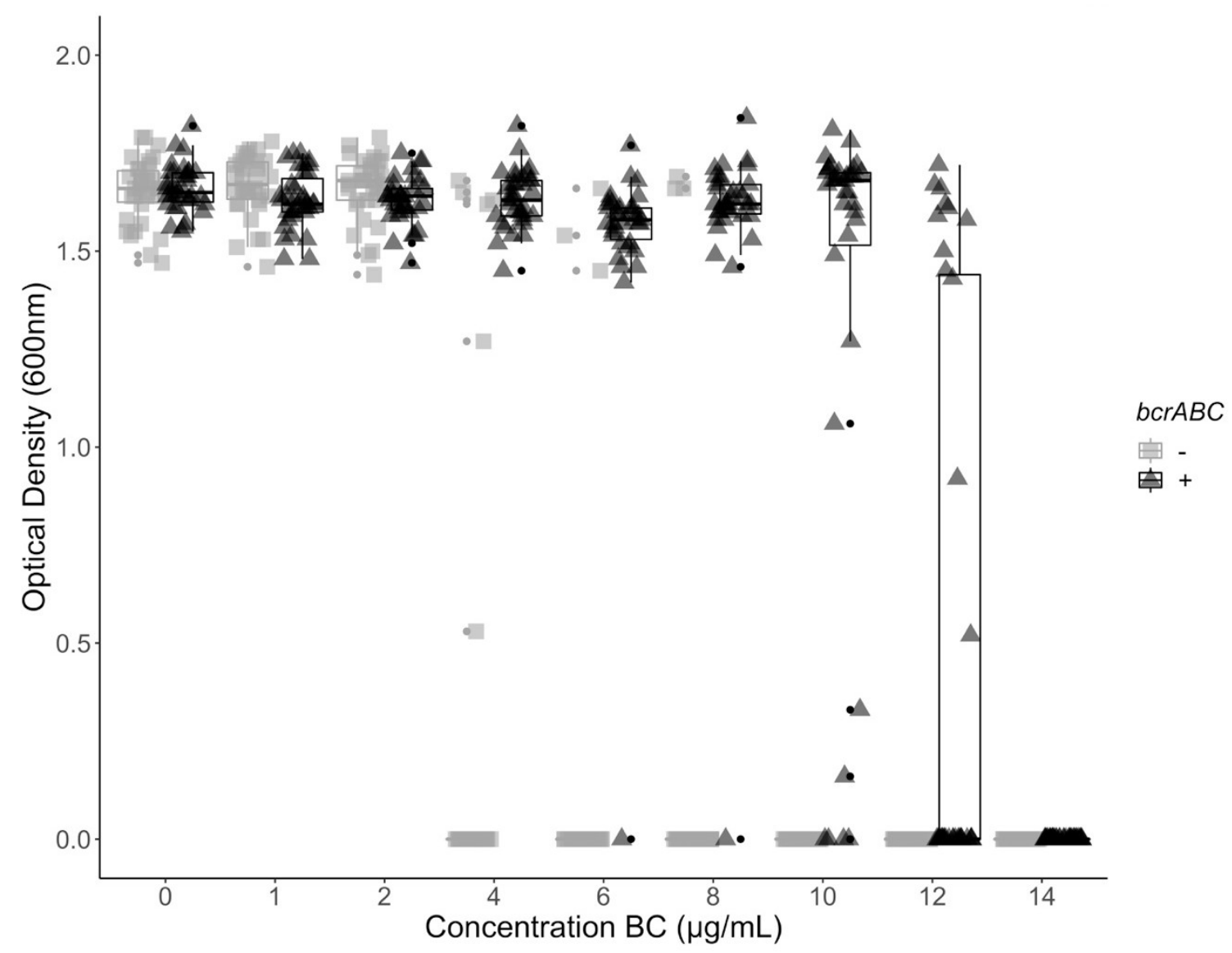

Figure 4.2. Phenotypic resistance to benzalkonium chloride $(\mathrm{BC})$ as a function of sanitizer resistance gene carriage. Thirty-five isolates with the $b c r A B C$ cassette $(\boldsymbol{\Delta})$ and 34 isolates without $(\square)$ were grown in the presence of increasing BC concentrations (x-axis). The optical density of cultures at each concentration was recorded following 48 $\mathrm{h}$ incubation (y-axis). Outliers are denoted above and/or below box plots as smaller dark circles. 


\subsubsection{Determination of $b c r A, b c r B$ and $b c r C$ by polymerase chain reaction}

The genetic profiles of $L$. monocytogenes strains for sanitizer resistance were determined primarily by WGS analysis; however, for food testing laboratories lacking access to sequencing equipment a more convenient approach for routine analysis of isolates would be to utilize a simple polymerase chain reaction (PCR) approach. We therefore examined the performance of a previously reported multiplex PCR targeting $b c r A, b c r B$ and $b c r C$ genes (Minarovičová et al., 2018) using the panel of strains presented in Appendix D.1 - Table 4.2. All 35 strains determined by WGS analysis to carry the $b c r A B C$ cassette produced PCR products of the expected size for each of the three genes (Appendix D.1 - Table 4.2), whereas none of the 34 strains in which the cassette was not identified produced detectable PCR products. This suggests that the PCR approach may be used in place of WGS analysis for rapid and cost-efficient determination of the $b c r A B C$ cassette in routine food isolates.

\subsubsection{Stability of plasmid-encoded $b c r A B C$ in foodborne $L$. monocytogenes isolates}

Our results indicate a wide distribution of the $b c r A B C$ cassette among $L$.

monocytogenes strains from different food commodities and manufacturing environments, and variability in its occurrence among different clonal complexes (Table 4.1). Nonetheless, the $b c r A B C$ cassette appeared to be more highly conserved in clonal complexes commonly observed in L. monocytogenes recovered from food testing programs. For example, all but one of the CC321 isolates harboured the $b c r A B C$ cassette. Likewise, Harrand et al. (2020) analysed isolates collected over 18 years from a cold- 
smoked salmon processing facility and found all CC321 $(n=33)$ in their study encoded bcrABC.

Notably, all CC321 isolates carried a similar plasmid according to Mob-recon analyses (Robertson and Nash, 2018), including the sole CC321 isolate that did not encode $b c r A B C$ (data not shown).

Other common CCs, such as CC5 and 155, had a somewhat lower overall rate of association with $b c r A B C$, though for some commodities such as meat (CC5), dairy and environmental (CC155) carriage rates ranged from 69-100\% (Table 4.1). Two clusters of CC5 isolates and one cluster of CC155 were identified in which the plasmid encoding the bcr $A B C$ cassette was variably present in epidemiologically related isolates which differed by only 0-1 high quality SNVs (Table 4.3). This may indicate lack of stability of the plasmid in certain genetic backgrounds. Correlations between CCs and $b c r A B C$ carriage was more difficult to ascertain for the less common CCs since the number of isolates available was much lower, making it difficult to arrive at any meaningful inference. Work is currently underway to investigate whether plasmid carriage is more stable in CC321 isolates relative to other clonal complexes. 
Table 4.3. Clusters of $L$. monocytogenes with variable presence of $b c r A B C$ among isolates $^{\mathrm{a}}$

\begin{tabular}{|c|c|c|c|c|}
\hline Cluster Description & Isolate ID & MLST & $\begin{array}{l}\text { Clonal } \\
\text { Complex }\end{array}$ & $\begin{array}{l}\text { Size of } \\
\text { genome }\end{array}$ \\
\hline $\begin{array}{c}\text { Isolates from butter } \\
\text { (O hqSNVs among isolates) }\end{array}$ & $\begin{array}{l}\text { CFIAFB20170041 } \\
\text { CFIAFB20170035 } \\
\text { CFIAFB20170031 } \\
\text { CFIAFB20170047 } \\
\text { CFIAFB20170042 } \\
\text { CFIAFB20170038 } \\
\text { CFIAFB20170043 } \\
\text { CFIAFB20170048 } \\
\text { CFIAFB20170037 } \\
\text { CFIAFB20170039 } \\
\text { CFIAFB20170049 } \\
\text { CFIAFB20170050 } \\
\text { CFIAFB20170030 } \\
\text { CFIAFB20170044 } \\
\text { CFIAFB20170036 } \\
\text { CFIAFB20170046 } \\
\text { CFIAFB20170040 } \\
\text { CFIAFB20170052 } \\
\text { CFIAFB20170051 } \\
\text { CFIAFB20170045 } \\
\text { CFIAFB20160019 }\end{array}$ & $\begin{array}{l}1093 \\
1093 \\
1093 \\
1093 \\
1093 \\
1093 \\
1093 \\
1093 \\
1093 \\
1093 \\
1093 \\
1093 \\
1093 \\
1093 \\
1093 \\
1093 \\
1093 \\
1093 \\
1093 \\
1093 \\
1093\end{array}$ & $\begin{array}{l}\text { CC5 } \\
\text { CC5 } \\
\text { CC5 } \\
\text { CC5 } \\
\text { CC5 } \\
\text { CC5 } \\
\text { CC5 } \\
\text { CC5 } \\
\text { CC5 } \\
\text { CC5 } \\
\text { CC5 } \\
\text { CC5 } \\
\text { CC5 } \\
\text { CC5 } \\
\text { CC5 } \\
\text { CC5 } \\
\text { CC5 } \\
\text { CC5 } \\
\text { CC5 } \\
\text { CC5 } \\
\text { CC5 }\end{array}$ & $\begin{array}{l}3032823 \\
3034878 \\
3036084 \\
3038573 \\
3114359 \\
3114402 \\
3115786 \\
3115888 \\
3115977 \\
3115983 \\
3116155 \\
3116158 \\
3116588 \\
3116592 \\
3116850 \\
3117144 \\
3117610 \\
3117654 \\
3118003 \\
3118768 \\
3150219\end{array}$ \\
\hline $\begin{array}{c}\text { Environmental isolates from a } \\
\text { RTE meat facility } \\
\text { (1 hqSNV among isolates) }\end{array}$ & $\begin{array}{l}\text { CFIAFB20130056 } \\
\text { CFIAFB20130057 }\end{array}$ & $\begin{array}{l}5 \\
5\end{array}$ & $\begin{array}{l}\text { CC5 } \\
\text { CC5 }\end{array}$ & $\begin{array}{l}3072751 \\
3152340\end{array}$ \\
\hline $\begin{array}{l}\text { RTE Meat and environmental } \\
\text { isolates from facility } \\
\text { (0 hqSNVs among isolates) }\end{array}$ & $\begin{array}{l}\text { CFIAFB20180157 } \\
\text { CFIAFB20180060 } \\
\text { CFIAFB20180061 } \\
\text { CFIAFB20180154 } \\
\text { CFIAFB20170078 } \\
\text { CFIAFB20180160 } \\
\end{array}$ & $\begin{array}{l}155 \\
155 \\
155 \\
155 \\
155 \\
155 \\
\end{array}$ & $\begin{array}{l}\text { CC155 } \\
\text { CC155 } \\
\text { CC155 } \\
\text { CC155 } \\
\text { CC155 } \\
\text { CC155 } \\
\end{array}$ & $\begin{array}{l}2885420 \\
2973852 \\
2970929 \\
2972569 \\
2973835 \\
2975385 \\
\end{array}$ \\
\hline
\end{tabular}

Abbreviations: CC, clonal complex; RTE, ready-to-eat; MLST, multi-locus sequence type; hqSNV, high quality single nucleotide variants.

${ }^{\text {a}}$ Isolates lacking $b c r A B C$ cassette highlighted in grey

\subsubsection{Carriage of QAC-resistance in clinically-relevant clonal complexes}

Historically, Canadian cases of listeriosis have been predominantly associated with CC8 L. monocytogenes (Knabel et al., 2012; Bergholz et al., 2018). The present study indicates that CC8 L. monocytogenes are also frequently isolated from foods 
(Figure 4.1). While the $b c r A B C$ cassette was uncommon in isolates of this lineage (Table 4.1), a high percentage of the CC8 isolates encoded emrE, and CC8 was significantly associated with QAC-resistance gene carriage (Figure 4.1). This correlates with previous reports of emrE in CC8 isolates from multiple outbreaks including the 2008 listeriosis outbreak in Canada (Knabel et al., 2012; Kovacevic et al., 2016; Bergholz et al., 2018).

Globally, listeriosis associated with previously uncommon clonal complexes 5, 6, 9, and 121 has been reported with increasing frequency (Bergholz et al., 2018). Interestingly a large increase in frequency of $\mathrm{CC} 5$, which is also the most abundant $\mathrm{CC}$ in this study, has been observed since 2000 (Bergholz et al., 2018). Of the 181 CC5 isolates in this study, 60\% carried a QAC resistance gene, with $b c r A B C$ being the most common (59.1\%), however this association was not statistically significant (Figure 4.1). Given the evidence of the variable presence of the plasmid encoding this cassette in clonal isolates with this genotype (Table 4.3), it is perhaps not surprising that only a subset of the CC5 isolates evaluated in this study harboured this cassette. Clonal complexes 6, 9 and 121 were also among the top 20 genotypes of the isolates in this study (Table S4.1). QAC resistance was frequently observed in CC9 and CC121, with 37\% (36 of 97) of the CC9 isolates encoding the $\operatorname{bcr} A B C$ cassette and $66.7 \%$ (22 of 33 ) of the CC121 isolates encoding the QAC resistance gene $q a c H$. In contrast, only 1 of 23 CC6 isolates carried QAC resistance genes. The association of QAC resistance with clinically relevant clonal complexes highlights the potential importance of this resistance for foodborne listeriosis. 


\subsection{Implementation of testing for sanitizer resistance in food testing programs}

Our results indicate a wide distribution of the $\operatorname{bcr} A B C$ cassette among $L$.

monocytogenes strains from different food commodities and manufacturing

environments, and its occurrence over a broad range of phylogenetically distinct isolates

(Table 4.1). Furthermore, carriage of an intact $b c r A B C$ cassette was generally correlated with phenotypic resistance to $\mathrm{BC}$, as was carriage of other QAC resistance genes, such as emrE (Figure 4.2). It is tempting to speculate that higher carriage of the cassette in meat products may be due to patterns of sanitizer use in those industries, but further study is needed to establish this. The presence of the cassette in a food or environmental isolate could be readily determined by WGS analysis or a simple PCR method targeting all three genes ( $b c r A, b c r B$ and $b c r C)$, the choice of method depending on the availability of resources in the food testing laboratory. It should be possible to develop PCR primers targeting any other sanitizer resistance genes of interest.

The presence of sanitizer resistance markers such as $b c r A B C$ in a laboratory isolate may warrant more intensive surveillance of the facility from which it originated (e.g., more frequent sampling of the plant environment and its food products, alternating sanitizers, verification of sanitization protocol efficacy), particularly for isolates bearing CCs known to be associated with human illness. Determination of a resistance marker could complement high resolution typing analyses of consecutive isolates from a manufacturing facility to establish whether persistent contamination is occurring, and this may in turn justify more intensive clean-up and auditing activities to ensure that appropriate risk mitigation actions have been taken. For this purpose, it may be possible to develop a practical risk management tool based on a weighted index integrating factors 
such as the prevalence of $b c r A B C$ (and other QAC resistance genes) among clonal types and their epidemiologic associations. Thus, the determination of $b c r A B C$ has the potential to provide a very useful adjunct to the arsenal of tools available to risk managers involved in food production and inspection seeking to mitigate the public health impacts of food contamination events.

While this study focused on the $b c r A B C$ cassette conferring resistance to benzalkonium chloride, the presence of other resistance genes such as qacC (Paulsen et al., 1995; Poole, 2005; Pirone-Davies et al., 2018), qacH (Müller et al., 2014; Ortiz et al., 2016) and emrE (Kovacevic et al., 2016) should also be considered in assessing whether a given L. monocytogenes isolate presents a heightened risk of persistence in food manufacturing environments. It has been suggested that even if the levels of quaternary ammonium compounds used for sanitation exceed the minimum inhibitory concentrations observed with strains carrying these genes, post-sanitation residue levels may remain sufficiently elevated to favor their survival (Møretrø et al., 2017). In any event, the establishment of routine WGS analysis of isolates in food testing laboratories opens the possibility of determining any number of relevant gene markers for the identification of potentially persistent strains. Further study is needed to identify the most useful gene markers for integration in analytical bioinformatics pipelines to this effect. 
Chapter 5: Foodborne Bacteria Facilitate Transmission and Persistence of Clinically Important Antimicrobial Resistance

This chapter will be published following additional data generation at OLC-CFIA. 


\section{$5.1 \quad$ Abstract}

Extended spectrum $\beta$-lactamase (ESBL)-producing Enterobacteriaceae are increasing in prevalence globally and are a major threat to public health. Transfer of mobile genetic elements (MGEs) encoding antimicrobial resistance (AMR) is widely thought to play a role in the spread of AMR genes (ARGs) between bacteria, including pathogens. The use of antimicrobials in agriculture and food production is potentially contributing to the development of AMR bacteria. Salmonella enterica, a member of the Enterobacteriaceae, are common foodborne pathogens carrying the $b l a_{\mathrm{CMY}-2}$ encoded ESBL which can be transmitted from food animals to humans. Although plasmid transfer is known to assist in the spread of AMR, little data exists examining the role of foodborne bacteria in the distribution of ARGs. We investigated the frequency of transfer of a conjugative IncI plasmid from a Salmonella ser. Heidelberg donor to 25 strains of common foodborne bacteria from 5 different genera including Citrobacter, Enterobacter, Hafnia, Klebsiella, and Proteus. Plasmid transfer frequency was calculated as CFU/mL of transconjugants/recipients. With the exception of Proteus mirabilis, most of the strains examined could receive the $b l a_{\mathrm{CMY}-2}$ encoding IncI plasmid. Different nutrient-sources and temperatures had minimal effects on plasmid transfer frequencies, though this was strain dependent. Conjugation frequencies were significantly higher for two isolates when enumerated on a lower concentration of CTX $(1 \mu \mathrm{g} / \mathrm{mL})$, and Hafnia strain Haf1237 transconjugants were unable to grow on $4 \mu \mathrm{g} / \mathrm{mL}$ CTX. All transconjugants stably maintained the acquired plasmid for at least 100 generations. These findings highlight the potential importance of food microbiota in transmission of clinically important AMR. 


\subsection{Introduction}

Antimicrobial use in medicine and agriculture is believed to be contributing to the development of antimicrobial resistance (AMR) by creating a selective pressure for persistence of AMR. Many environments including plants, animals, and water sources can function as routes for transfer of AMR genes (ARGs) between bacterial populations (Huijbers et al., 2015; Bengtsson-Palme, 2017). Food production connects many of these habitats and has the potential to further spread of AMR and pathogenic bacteria (Bengtsson-Palme, 2017). Therefore, understanding the ecology and transmission properties of AMR in agri-food production has become increasingly important for surveillance. Currently AMR surveillance mostly targets individual bacterial strains through isolation and phenotyping or sequencing, or within animals investigating the percentage of AMR bacterial isolates in animal species (Ricker et al., 2020).

Extended spectrum-ß-lactamase (ESBL) producing Enterobacteriaceae are on the World Health Organization (WHO) list of serious threats, and infections have been increasing in Canada (Lob et al., 2016; Denisuik et al., 2019b,a; Lagacé-Wiens et al., 2019) and the United States (Lob et al., 2016; CDC, 2017b). Of particular concern are strains of Salmonella enterica, Klebsiella pneumoniae, and Escherichia coli carrying multidrug resistance plasmids encoding carbapenemases and ESBLs (Porse et al., 2016; Tran et al., 2020).

Significant increases in the prevalence of AMR Salmonella enterica, a major cause of foodborne infection, have been observed (Guo et al., 2014; Kuang et al., 2018; Government of Canada, 2020; Tran et al., 2020). A 2.9\% increase in non-typhoidal Salmonella resistance to third generation cephalosporins was observed from 2014 to 2018 
in Canada (Government of Canada, 2020). ESBLs are often encoded on plasmids which may be transferred between bacteria (Aviv et al., 2016; Ben Said et al., 2016; Saliu et al., 2020). IncI replicon types carrying $b a_{\mathrm{CMY}-2}$ have been associated with farm animals in Canada (Martin et al., 2012; Moffat et al., 2020). Expansive spread of IncI plasmids carrying bla $a_{\mathrm{CMY}-2}$ have been reported in E. coli (Folster et al., 2011; Sidjabat et al., 2014) and Salmonella (Guo et al., 2014; Moffat et al., 2020), and wide distribution of plasmids encoding bla $a_{\mathrm{CMY}-2}$ among Escherichia coli and Salmonella isolates across Canada may be drivers of observed increases in ESBL resistance (Mataseje et al., 2010; Martin et al., 2012; Cejas et al., 2014; Edirmanasinghe et al., 2017).

Bacteria in all environments can develop AMR through horizontal gene transfer (HGT) mechanisms such as conjugation, transduction, and transformation (Hudson et al., 2017). Gene transfer between different genera and species often occurs via conjugation, as mobile genetic elements (MGE) such as plasmids often harbour genes such as ARGs that confer a selective advantage (Davison, 1999; Wintersdorff et al., 2016). Thus, conjugation is important in bacterial evolution, enabling acquisition of resistance and virulence phenotypes which may permit environmental adaptations (Aviv et al., 2016; Dimitriu et al., 2019). Consequently successful HGT can impact competition between bacteria increasing survival of strains in environmental and clinical settings (Heuer and Smalla, 2012; Conlan et al., 2016; Porse et al., 2017; Baker et al., 2018; Dimitriu et al., 2019).

Conjugative plasmid transfer rates can vary depending on a number of factors including donor-recipient relatedness (Hardiman et al., 2016; Cairns et al., 2018; Dimitriu et al., 2019; Alderliesten et al., 2020), substrate, and environmental conditions 
such as temperature (Aviv et al., 2016; Hardiman et al., 2016; Bengtsson-Palme et al., 2018). Aviv et al. (2016) demonstrated conjugative transfer of a plasmid between Salmonella enterica serovar Infantis and Escherichia coli occurs at higher rates in conditions characterizing the intestinal environment of warm-blooded animals. Many studies have investigated plasmid transfer within clinical isolates (Sarowska et al., 2009; Franiczek et al., 2010; Hardiman et al., 2016; Knudsen et al., 2018), in artificial bacterial communities (Cairns et al., 2018), between manure and soil communities (Smalla et al., 2000; Binh et al., 2008; Klümper et al., 2015; Westhoff et al., 2017; Kottara et al., 2020), or to common laboratory strains (Licht et al., 1999; Winokur et al., 2001; Kim et al., 2005; Franiczek et al., 2010; Hu et al., 2012; Dimitriu et al., 2019). Yet, laboratoryadapted strains may be poor models for natural bacterial populations, and conjugation data is lacking for bacterial species from food and food production sources. In order to better understand the role of foodborne bacteria in dissemination of AMR, we need to understand the transmission dynamics between foodborne bacteria.

There is evidence of $b l a_{\mathrm{CMY}-2} \mathrm{ARG}$ presence in bacterial genera commonly observed in food products such as Citrobacter, Enterobacter, Klebsiella and Proteus species (Table 5.1). Saliu et al. (2020) reported transfer of ESBL-producing plasmids from E. coli to bacteria including Enterobacter cloacae, E. coli, Serratia marcescens, and Salmonella ser. Typhimurium which are commonly detected in the gastrointestinal tract of poultry. As the majority of studies have investigated the transmission of plasmids between clinical isolates and/or laboratory E. coli strains, there is a potential role of food microbiota as a reservoir of MGEs and intermediators in the spread of AMR that has not been well characterised. In this study we investigated factors, such as temperature and 
nutrient availability, that may influence conjugation of a clinically relevant plasmid to foodborne bacterial isolates. We provide evidence that transfer of AMR plasmids to food commensal bacteria occurs at high frequency, for some strains even at $25^{\circ} \mathrm{C}$ with low nutrient levels, and that foodborne bacteria can act as a reservoir for AMR of clinical importance.

Table 5.1. Bacterial genera, source(s) and location(s) where bla $a_{\mathrm{CMY}-2}$ has been reported

\begin{tabular}{|c|c|c|c|c|}
\hline Organism & Type & Location(s) & $\begin{array}{l}\text { Isolation } \\
\text { Source(s) }\end{array}$ & References \\
\hline Citrobacter spp. & Clinical & Taiwan & Humans & $\begin{array}{l}\text { (Chuang et al., 2006; } \\
\text { Su et al., 2006) }\end{array}$ \\
\hline \multirow[t]{2}{*}{ Enterobacter spp. } & Clinical & $\begin{array}{l}\text { Greece, Taiwan, } \\
\text { Iran, Cambodia, } \\
\text { Algeria }\end{array}$ & $\begin{array}{l}\text { Humans, } \\
\text { bloodstream } \\
\text { infections }\end{array}$ & $\begin{array}{l}\text { (Philippon et al., 2002; } \\
\text { Su et al., 2006; } \\
\text { Labadene et al., 2009; } \\
\text { Vlieghe et al., 2015; } \\
\text { Peymani et al., 2016) }\end{array}$ \\
\hline & $\begin{array}{l}\text { Agriculture/ } \\
\text { Animal }\end{array}$ & Canada & $\begin{array}{l}\text { Turkey farm } \\
\text { fecal samples }\end{array}$ & (Moffat et al., 2020) \\
\hline \multirow[t]{2}{*}{ Escherichia coli } & Clinical & $\begin{array}{l}\text { Thailand, Greece, } \\
\text { Portugal, Taiwan, } \\
\text { Canada, Korea, } \\
\text { Taiwan - Asia- } \\
\text { Pacific, Spain, } \\
\text { USA, Japan, Bosnia } \\
\text { and Herzegovina, } \\
\text { Cambodia, Algeria, } \\
\text { Egypt }\end{array}$ & $\begin{array}{l}\text { blood, urine, } \\
\text { pus, body fluid, } \\
\text { sputum, ICU, } \\
\text { Nosocomial } \\
\text { infections, intra- } \\
\text { abdominal, pus, } \\
\text { urine, blood, } \\
\text { drain, graft, bile, } \\
\text { percutaneous, } \\
\text { UTI, } \\
\text { bloodstream } \\
\text { infection }\end{array}$ & $\begin{array}{l}\text { (Navarro et al., 2001; } \\
\text { Philippon et al., 2002; } \\
\text { Labadene et al., 2009; } \\
\text { Doi et al., 2010; } \\
\text { Singtohin et al., 2010; } \\
\text { Yamasaki et al., 2010; } \\
\text { Rodríguez-Baño et al., } \\
\text { 2012; Park et al., } \\
\text { 2013; Sheng et al., } \\
\text { 2013; Helmy and } \\
\text { Wasfi, 2014; } \\
\text { Ibrahimagić et al., } \\
\text { 2015; Manageiro et } \\
\text { al., 2015; Vlieghe et } \\
\text { al., 2015) }\end{array}$ \\
\hline & $\begin{array}{l}\text { Agriculture/ } \\
\text { Animal }\end{array}$ & $\begin{array}{l}\text { Canada, } \\
\text { Switzerland, Spain, } \\
\text { USA, Austria, } \\
\text { Japan, Netherlands, } \\
\text { France }\end{array}$ & $\begin{array}{l}\text { Feedlot cattle, } \\
\text { retail meat } \\
\text { products } \\
\text { (wings), raw } \\
\text { poultry, retail } \\
\text { meat, retail } \\
\text { meat, avian, } \\
\text { Broiler farms, } \\
\text { companion } \\
\text { animals, turkey }\end{array}$ & $\begin{array}{l}\text { (Forward et al., 2004; } \\
\text { Doi } \text { et al., 2010; } \\
\text { Mataseje et al., 2010; } \\
\text { Dierikx et al., 2013; } \\
\text { Kameyama et al., } \\
\text { 2013; Loncaric et al., } \\
\text { 2013; Seiffert } \text { et al., } \\
\text { 2013; Haenni et al., } \\
\text { 2014; Moffat et al., } \\
\text { 2020) }\end{array}$ \\
\hline
\end{tabular}




\begin{tabular}{|c|c|c|c|c|}
\hline & Water & Canada, Tunisia & $\begin{array}{l}\text { Drinking water } \\
(\mathrm{AB}, \mathrm{QB}, \mathrm{ON}) \text {, } \\
\text { Wastewater and } \\
\text { surface water }\end{array}$ & $\begin{array}{l}\text { (Mataseje et al., 2010; } \\
\text { Ben Said et al., 2016) }\end{array}$ \\
\hline Klebsiella oxytoca & Clinical & $\begin{array}{l}\text { Taiwan - Asia- } \\
\text { Pacific, Japan, } \\
\text { Spain, Egypt, } \\
\text { Bosnia and } \\
\text { Herzegovina }\end{array}$ & $\begin{array}{l}\text { intra-abdominal, } \\
\text { extract from } \\
\text { percutaneous } \\
\text { transhepatic } \\
\text { cholangial } \\
\text { drainage, UTI }\end{array}$ & $\begin{array}{l}\text { (Navarro et al., 2001; } \\
\text { Yamasaki et al., 2010; } \\
\text { Sheng et al., 2013; } \\
\text { Helmy and Wasfi, } \\
\text { 2014; Ibrahimagić et } \\
\text { al., 2015) }\end{array}$ \\
\hline \multirow[t]{2}{*}{$\begin{array}{l}\text { Klebsiella } \\
\text { pneumoniae }\end{array}$} & Clinical & $\begin{array}{l}\text { Thailand, Greece, } \\
\text { Taiwan, Korea, } \\
\text { Taiwan - Asia- } \\
\text { Pacific, Spain, } \\
\text { Cambodia, Algeria }\end{array}$ & $\begin{array}{l}\text { blood, urine, } \\
\text { pus, body fluid, } \\
\text { sputum, intra- } \\
\text { abdominal }\end{array}$ & $\begin{array}{l}\text { (Bauernfeind } \text { et al., } \\
\text { 1996; Navarro et al., } \\
\text { 2001; Philippon et al., } \\
\text { 2002; Su et al., 2006; } \\
\text { Labadene et al., 2009; } \\
\text { Singtohin et al., 2010; } \\
\text { Rodríguez-Baño et al., } \\
\text { 2012; Park et al., } \\
\text { 2013; Sheng et al., } \\
\text { 2013; Vlieghe et al., } \\
\text { 2015) }\end{array}$ \\
\hline & $\begin{array}{l}\text { Agriculture/ } \\
\text { Animal }\end{array}$ & Canada & $\begin{array}{l}\text { Turkey farm } \\
\text { fecal samples }\end{array}$ & (Moffat et al., 2020) \\
\hline Morganella spp. & Clinical & $\begin{array}{l}\text { Taiwan, Taiwan - } \\
\text { Asia-Pacific }\end{array}$ & intra-abdominal & $\begin{array}{l}\text { (Su et al., 2006; Sheng } \\
\text { et al., 2013) }\end{array}$ \\
\hline \multirow[t]{2}{*}{ Proteus spp. } & Clinical & $\begin{array}{l}\text { Taiwan, Bosnia and } \\
\text { Herzegovina, } \\
\text { Korea, Taiwan - } \\
\text { Asia-Pacific, Spain, } \\
\text { Japan, Tunisia, } \\
\text { Algeria }\end{array}$ & $\begin{array}{l}\text { intra-abdominal, } \\
\text { urine, urine }\end{array}$ & $\begin{array}{l}\text { (Verdet et al., 1998; } \\
\text { Navarro et al., 2001; } \\
\text { Su et al., 2006; } \\
\text { Labadene } \text { et al., 2009; } \\
\text { Yamasaki et al., 2010; } \\
\text { Rodríguez-Baño et al., } \\
\text { 2012; Park et al., } \\
\text { 2013; Sheng et al., } \\
\text { 2013; Ibrahimagić et } \\
\text { al., 2015; Uzunović et } \\
\text { al., 2016) }\end{array}$ \\
\hline & $\begin{array}{l}\text { Agriculture/ } \\
\text { Animal }\end{array}$ & $\begin{array}{l}\text { Canada, China, } \\
\text { Switzerland, Hong } \\
\text { Kong, Netherlands }\end{array}$ & $\begin{array}{l}\text { chicken, raw } \\
\text { poultry meat, } \\
\text { chicken carcass, } \\
\text { companion } \\
\text { animals, turkey } \\
\text { farm fecal } \\
\text { samples }\end{array}$ & $\begin{array}{l}\text { (Dierikx et al., 2013; } \\
\text { Seiffert et al., 2013; } \\
\text { Wong et al., 2013; Lei } \\
\text { et al., 2017; Moffat et } \\
\text { al., 2020) }\end{array}$ \\
\hline
\end{tabular}

(Su et al., 2006;

Labadene et al., 2009) 


\begin{tabular}{|c|c|c|c|c|}
\hline \multirow[t]{2}{*}{ Salmonella spp. } & Clinical & \multicolumn{2}{|l|}{$\begin{array}{l}\text { France (Algeria), } \\
\text { USA, Taiwan, } \\
\text { Canada, Spain }\end{array}$} & $\begin{array}{l}\text { (Navarro et al., 2001; } \\
\text { Philippon et al., 2002; } \\
\text { Carattoli, 2003; Su et } \\
\text { al., 2006; Mataseje et } \\
\text { al., 2010) }\end{array}$ \\
\hline & $\begin{array}{l}\text { Agriculture/ } \\
\text { Animal }\end{array}$ & $\begin{array}{l}\text { USA, Canada, } \\
\text { France }\end{array}$ & $\begin{array}{l}\text { cattle, pigs, } \\
\text { retail meat } \\
\text { (wings), dogs }\end{array}$ & $\begin{array}{l}\text { (Philippon et al., 2002; } \\
\text { Forward et al., 2004; } \\
\text { Haenni et al., 2014) }\end{array}$ \\
\hline Serratia spp. & Clinical & Algeria & & $\begin{array}{l}\text { (Su et al., 2006; } \\
\text { Labadene } \text { et al., 2009) }\end{array}$ \\
\hline Shigella & Clinical & Taiwan & & (Su et al., 2006) \\
\hline
\end{tabular}

\subsection{Materials and Methods}

\subsubsection{Growth conditions}

Bacteria were grown at $36^{\circ} \mathrm{C}$ on Universal Differential ChromoSelect Medium (UChrom) (Millipore Sigma, Ontario, Canada), Mueller Hinton agar (MHA) (Oxoid, Ontario, Canada), Lysogeny-Broth (LB)-Lennox agar (LBA) (BD Difco, Ontario, Canada), or in LB-Lennox broth (BD Difco, Ontario, Canada). Where indicated media was supplemented with cefotaxime (CTX) (1 or $4 \mu \mathrm{g} / \mathrm{mL})$, and/or rifampin (Rif) (100 $\mu \mathrm{g} / \mathrm{mL}$ ) (Fisher Scientific: TCI America, Ontario, Canada), or nalidixic acid (NAL) (25 $\mu \mathrm{g} / \mathrm{mL}$ ) for $E$. coli recipient $\mathrm{MG} 1655$. For mating experiments, conjugation mixtures were spread on minimal salts agar (M9A) or LB-Lennox agar (LBA) and incubated at $36^{\circ} \mathrm{C}$ or room temperature $\left(25^{\circ} \mathrm{C}\right)$. M9A was prepared as follows: M9, Minimal Salts, $5 \mathrm{X}$ powder (Millipore Sigma, Ontario, Canada) was suspended in $250 \mathrm{~mL}$ of water and autoclaved. Simultaneously, $7.5 \mathrm{~g}$ of Bacteriological agar (Millipore Sigma, Ontario, Canada) was dissolved in $250 \mathrm{~mL}$ of water and autoclaved. Sterilized M9 salts and agar solutions were combined once tempered to $50^{\circ} \mathrm{C}$, then $10 \mathrm{~mL}$ of $20 \%$ glucose (Millipore 
Sigma, Ottawa, Canada), $1 \mathrm{~mL}$ of $1 \mathrm{M} \mathrm{MgSO}_{4}$, and $50 \mu \mathrm{L}$ of $1 \mathrm{M} \mathrm{CaCl}_{2}$ were aseptically added to mixture before pouring into petri dishes.

\subsubsection{Bacterial Strains and Plasmids}

The Salmonella enterica serovar Heidelberg donor strain was isolated from chicken as part of the national Microbiology Baseline Study (MBS) in Broiler Chickens (Government of Canada, 2016) (Table 5.2). Donor WGS raw-read data is available through the sequence read archive (SRA) under sample run identifier SRR10859129 (biosample: SAMN13334284).

Table 5.2. Bacterial donor strain, plasmid types, and antimicrobial resistance genes

\begin{tabular}{lllll}
\hline Isolate ID & Species & $\begin{array}{l}\text { Plasmid Incompatibility } \\
\text { Group(s) }\end{array}$ & $\begin{array}{l}\text { Plasmid } \\
\text { Length (bp) }\end{array}$ & ARG(s) \\
\hline OLC2644 & $\begin{array}{l}\text { Salmonella } \text { ser. } \\
\text { Heidelberg }\end{array}$ & IncI & 98976 & blaCMY-2 \\
& & IncX1 & 37697 & \\
& & ColRNAI & 5880 & \\
\hline
\end{tabular}

Abbreviations: bp, base pair; ARG, antimicrobial resistance gene; ser., serovar.

\subsubsection{DNA Extraction}

For recipient strains isolated from food products, a single colony was suspended in $1 \mathrm{~mL}$ of brain heart infusion (BHI) broth and incubated overnight at $37^{\circ} \mathrm{C}$. Genomic DNA was isolated from overnight broth cultures using the Promega Maxwell 16 Cell DNA purification kit (Promega, WI, USA). For each sample, $400 \mu \mathrm{L}$ of broth culture was added to a Maxwell SEV cartridge and extracted per manufacturer's instructions. Extracted DNA was transferred to $1.5 \mathrm{~mL}$ micronic tubes and stored at $-20^{\circ} \mathrm{C}$ until 
sequencing. Remaining cultures were mixed with an equal volume of $30 \%$ glycerol and frozen at $-80^{\circ} \mathrm{C}$ for long term storage.

\subsubsection{Whole-genome Sequencing (WGS)}

Paired-end Illumina MiSeq short reads were processed with the CFIA-OLC Workflow for Bacterial Assembly and Typing (COWBAT) as described previously (Chapter 1, Cooper et al. 2020), with the exception of plasmid analysis and typing which was conducted using MOB-suite version 3.0.0 (Robertson and Nash, 2018).

\subsubsection{WGS Screening of Candidate Recipient Strains for AMR and Plasmids}

An Escherichia coli K-12 nalidixic-acid resistant (NalR) isolate MG1655 was included as a control strain as it is commonly used in conjugation studies (Franiczek et al., 2010; Aviv et al., 2016; Hardiman et al., 2016). Bacterial recipient strains were selected to include a variety of species that have been associated with clinical infections and are often isolated from foods (Table 5.3). A number of Klebsiella, Enterobacter, Citrobacter, Hafnia, and Proteus species isolated from food products and sequenced at the Ottawa Laboratory Carling Canadian Food Inspection Agency (OLC-CFIA) were screened to select recipient strains (data not shown). To ensure donor resistance was not present in recipients, candidate recipient strains for each species were first narrowed based on ARG and plasmid contents detected in whole genome sequence (WGS) data. Sequences for candidate species were analysed using the KMA ( $k$-mer alignment) tool version 1.17 (Clausen et al., 2018) with the NCBI Bacterial Antimicrobial Resistance Reference Gene database (bioproject PRJNA313047, downloaded on November 11, 
2019) to determine which isolates harboured ARGs that would confer resistance to third generation cephalosporins, possibly interfering with transconjugant enumeration, and these were removed from the candidate list. Following ARG determination, MOB-suite version 3.0.0 (Robertson and Nash, 2018) was used to reconstruct and type plasmids in candidate recipients in order to minimize inclusion of recipient isolates harbouring incompatibility groups matching those of the donor plasmids where possible.

Table 5.3. Bacterial recipient strains, plasmid types, and antimicrobial resistance gene(s)

\begin{tabular}{|c|c|c|c|c|}
\hline Recipient ID & Species & Isolation Source & $\mathrm{ARG}(\mathrm{s})$ & ${\operatorname{Plasmid}(\mathrm{s})^{\mathrm{a}}}^{\mathrm{a}}$ \\
\hline Cit1135 & Citrobacter freundii & Raw ground beef & $\begin{array}{l}b l a_{\mathrm{CMY}-65}, \\
\operatorname{aac}\left(6^{\prime}\right)-I f, \\
q n r B\end{array}$ & ColRNAI \\
\hline Cit1224 & Citrobacter freundii & Medium ground beef & $\begin{array}{l}\text { bla }_{\mathrm{CMY}-135,} \\
\operatorname{aac}\left(6^{\prime}\right)-I f, \\
q n r B 10\end{array}$ & IncN, IncR \\
\hline Cit2431 & Citrobacter braakii & Ground beef & $b l a_{\mathrm{CMY}-70}$ & \\
\hline Cit2915 & Citrobacter youngae & Sprout & $b l a_{\mathrm{CMY}-53}$ & \\
\hline Cit3215 & Citrobacter sp. & Feed & $\begin{array}{l}\text { bla }_{C M Y-159,} \\
q n r B\end{array}$ & \\
\hline Ent1650 & Enterobacter cloacae & Baby spinach & $\begin{array}{l}b a_{\mathrm{ACT}-23,} \\
\text { fos } A, \text { oqxA, } \\
\text { oqxB }\end{array}$ & \\
\hline Ent2448 & Enterobacter & Unknown Food & $\begin{array}{l}\text { bla } a_{\mathrm{ACT}-27,} \\
\text { fos } A, \text { oqxA, } \\
\text { oqxB }\end{array}$ & $\begin{array}{l}\text { IncFIB, } \\
\text { ColRNAI }\end{array}$ \\
\hline Ent2920 & Enterobacter cloacae & Cheese & $\begin{array}{l}b^{l} a_{\mathrm{ACT}-37} \\
o q x A, o q x B\end{array}$ & ColRNAI \\
\hline Ent2939 & Enterobacter cloacae & Ground veal & $\begin{array}{l}b l a_{\text {АCT-43, }} \\
o q x A, \text { fos } A\end{array}$ & \\
\hline Ent3235 & Enterobacter cloacae & Feed & $\begin{array}{l}\text { bla } a_{\mathrm{ACT}-41}, \\
\text { fos } A, \text { oq } x A,\end{array}$ & \\
\hline
\end{tabular}




\begin{tabular}{|c|c|c|c|c|}
\hline \multirow[b]{2}{*}{ Haf1231 } & \multirow[b]{2}{*}{ Hafnia alvei } & \multicolumn{3}{|c|}{$o q x B$} \\
\hline & & Beef trim & $b l a_{\mathrm{ACC}-1 \mathrm{~d}}$ & ColRNAI \\
\hline Haf1276 & Hafnia alvei & Beef trim & $b l a_{\mathrm{ACC}-1 \mathrm{a}}$ & ColRNAI \\
\hline Haf1306 & Hafnia alvei & Veal & $b l a_{\mathrm{ACC}-1 \mathrm{a}}$ & ColRNAI \\
\hline Haf1525 & Hafnia alvei & Beef trim & $b l a_{\mathrm{ACC}-5}$ & $\begin{array}{l}\text { IncX7, } \\
\text { IncFII, } \\
\text { ColRNAI }\end{array}$ \\
\hline Haf3292 & Hafnia alvei & Veal & $b l a_{\mathrm{ACC}-1 \mathrm{a}}$ & ColRNAI \\
\hline Klb1227 & Klebsiella pneumoniae & Raw ground beef & $\begin{array}{l}\text { bla }_{\mathrm{SHV}-164}, \\
\text { oqx } B, \text { oq } x A, \\
\text { fos } A\end{array}$ & $\begin{array}{l}\text { IncFIB, } \\
\text { IncFII, } \\
\text { ColRNAI }\end{array}$ \\
\hline Klb1237 & Klebsiella pneumoniae & Beef trim & $\begin{array}{l}\text { bla }_{\mathrm{SHV}-110} \\
\text { oqx } B, \text { oqx } A, \\
\text { fos } A\end{array}$ & $\begin{array}{l}\text { IncFIB, } \\
\text { ColRNAI }\end{array}$ \\
\hline Klb1651 & Klebsiella pneumoniae & Beef trim & $\begin{array}{l}\text { bla }_{\mathrm{SHV}-40}, \\
\text { oqx } B, \text { oq } x A \text {, } \\
\text { fos } A\end{array}$ & $\begin{array}{l}\text { IncFIB, } \\
\text { IncFII, } \\
\text { ColRNAI }\end{array}$ \\
\hline Klb1672 & Klebsiella pneumoniae & Raw ground beef & $\begin{array}{l}\text { bla }_{\mathrm{SHV}-36} \\
o q x B, \text { oqxA }\end{array}$ & $\begin{array}{l}\text { IncFII, } \\
\text { IncR, } \\
\text { ColRNAI }\end{array}$ \\
\hline Klb2894 & Klebsiella pneumoniae & Sprouts & $\begin{array}{l}\text { bla }{ }_{\mathrm{SHV}-172}, \\
\text { oqx } B, \text { oqx } A, \\
\text { fos } A\end{array}$ & ColRNAI \\
\hline Prt1100 & Proteus mirabilis & Finely textured beef & $\operatorname{cat} A 4, \operatorname{tet}(J)$ & ColRNAI \\
\hline Prt1615 & Proteus mirabilis & ATCC25933 & $\operatorname{cat} A 4, \operatorname{tet}(J)$ & \\
\hline Prt2451 & Proteus mirabilis & Environmental swab & $\operatorname{catA4}, \operatorname{tet}(J)$ & \\
\hline Prt3112 & Proteus mirabilis & Veal & $\begin{array}{l}\operatorname{cat} A 4, \operatorname{aad} A 1, \\
d \operatorname{fr} A 1, \operatorname{tet}(J)\end{array}$ & \\
\hline $\operatorname{Prt} 3244$ & Proteus mirabilis & Beef heart & $\operatorname{cat} A 4, \operatorname{tet}(J)$ & \\
\hline MG1655 ${ }^{\mathrm{b}}$ & Escherichia coli & Laboratory strain & & \\
\hline
\end{tabular}




\subsubsection{Creation of rifampicin-resistant mutants for conjugation experiments}

Following candidate screening above, recipient strains were selected

bioinformatically using StrainChoosr to determine the 5 most genetically diverse isolates in the narrowed subset (Available at: https://github.com/OLC-

Bioinformatics/StrainChoosr ) (Pardi and Goldman, 2005; Steel, 2005). Briefly, StrainChoosr uses Parsnp (Treangen et al., 2014) to create a phylogenetic tree of selected isolate sequences based on their core genomes. A distance matrix is then created determining the distance (relationship) between each of the isolates in the tree. The most diverse isolates are then selected based on the distance matrix, and a list of $n$ isolates is output based on user's criteria. For this study $n$ was set to five isolates for each of the genera tested.

To create rifampicin resistant (RifR) recipients, parent isolates were grown overnight at $37^{\circ} \mathrm{C}$ in $10 \mathrm{~mL}$ of LB broth. Broth cultures were centrifuged at $11,000 \mathrm{x} \mathrm{g}$ at $4^{\circ} \mathrm{C}$ for 2 min. to concentrate bacterial cells. The supernatant was then discarded, and pellet was plated on LBA containing $100 \mu \mathrm{g} / \mathrm{mL}$ Rif(LBA + Rif) to select for RifR mutants. Plates were incubated overnight at $37^{\circ} \mathrm{C}$, and RifR mutants were purified the next day by streaking single colonies onto LBA + Rif.

\subsubsection{Evaluation of RifR Retention and Fitness}

RifR mutants were compared to parent strains to ensure RifR mutations were stably maintained and did not impose significant fitness costs. Mutants and parent strains were grown overnight in one $\mathrm{mL}$ of LB broth at $37^{\circ} \mathrm{C}$. Overnight cultures were diluted in sterile PBS and plated on LBA (parent and mutant growth) and LBA + Rif (only mutants 
grow) and incubated overnight at $37^{\circ} \mathrm{C}$. Counts of colony forming units (CFU) per $\mathrm{mL}$ of broth were calculated on LBA and LBA + Rif for mutants and compared to CFU $/ \mathrm{mL}$ of parent strain on LBA.

\subsubsection{Antimicrobial Susceptibility Testing}

Minimum inhibitory concentrations of Rif and CTX were determined for all isolates prior to mating experiments and in transconjugants following successful conjugation. All donors, RifR mutant recipients, and Escherichia coli ATCC 25922 (sensitive control) were streaked for single colonies onto MHA and incubated overnight at $37^{\circ} \mathrm{C}$. Antibiotics were diluted in MHA at concentrations of $0,0.5,1,2,4$, and 8 $\mu \mathrm{g} / \mathrm{mL}$ for CTX, and $0,16,25,50$, and $100 \mu \mathrm{g} / \mathrm{mL}$ for Rif. All antibiotic-containing MHA plates were dried in an incubator at $37^{\circ} \mathrm{C}$ for at least 30 min prior to experimentation. A 0.5 McFarland suspension of each isolate was made using colonies from overnight growth plates, then diluted 1:10 in SPBS. A 48-pin replicator was used to spot $1 \mu \mathrm{L}$ aliquots on dried MHA containing antibiotics moving from lowest to highest concentration. Duplicates were plated for each concentration. All plates were incubated overnight at $37^{\circ} \mathrm{C}$. The MIC for each isolate was recorded as the lowest concentration of antibiotic that completely inhibited growth.

\subsubsection{Multiplex PCR (mPCR) for Plasmid Recipient Verification}

Transconjugants were confirmed by colony morphology on Universal Differential ChromoSelect Medium (UChrom) (Millipore Sigma, ON, Canada) and multiplex PCR (mPCR) following mating experiments. Primers (Integrated DNA Technologies, IL, 
USA) were used to screen for donor strain (OLC2644), the selected plasmid-borne ARG bla $a_{\mathrm{CMY}-2}$, and the small multidrug resistance efflux gene $\operatorname{sug} E$ and outer membrane lipoprotein gene $b l c$ located adjacent to $b l a_{\mathrm{CMY}-2}$ (Cejas et al., 2014) (Table 5.4). Specific primers for the bacterial donor were created using an in-house bioinformatic tool, SigSeekr (available at: https://github.com/OLC-Bioinformatics/SigSeekr) (Knowles et al., 2015), using the donor WGS as the inclusion group and recipient WGSs as the exclusion group. Briefly, to find sequences, SigSeekr splits both inclusion and exclusion groups into $k$-mers of size 31 . It then filters out $k$-mers from the inclusion group that are also found in the exclusion group and uses the remaining inclusion $k$-mers to form longer contiguous sequences by aligning these $k$-mers back to one of the inclusion genomes. These contiguous sequences are then blasted against all inclusion genomes to make sure that the sequence is present in all inclusion genomes, and validated sequences are then run through primer3 to generate primers. Analysis of these primers using NCBI primer blast found isolate-primers hit other sequences in the NCBI database of the same species and were therefore not strain-specific. However, as recipients were used as exclusions during primer creation and are mostly of different species, this was not of concern for this study.

Primers for the $b l a_{\mathrm{CMY}-2}$ gene were designed in house as follows: (1) nucleotide sequences for alleles of $b l a_{\mathrm{CMY}-2}$ were downloaded from the Center for Genomic Epidemiology (CGE) ResFinder database (Accessible at: https://bitbucket.org/genomicepidemiology/resfinder_db); (2) alleles for bla $a_{\mathrm{CMY}-2}$ were aligned and analysed for conserved sequence regions; (3) conserved regions were 
analysed through NCBI Primer-BLAST to design target-specific primers for use in PCR (Ye et al., 2012).

Table 5.4. Primers used for transconjugant confirmation

\begin{tabular}{|c|c|c|c|c|}
\hline Target & & Primer Sequence (5' to 3') & $\begin{array}{l}\text { Amplicon } \\
\text { Size }\end{array}$ & Reference \\
\hline Donor & $\mathrm{F}$ & ACTCGGAACTGACCTCCAGA & 104 & This study \\
\hline OLC2644 & $\mathrm{R}$ & TGCAAGTGTGAGCGATGTCT & & \\
\hline bla $_{\mathrm{CMY}-2}$ & $\mathrm{~F}$ & CCGCTGAAAGCTGATTCGATCATCA & 220 & This study \\
\hline & $\mathrm{R}$ & CACGGACAGGGTTAGGATAGCTTTTGT & & \\
\hline$b l c-F$ & $\mathrm{~F}$ & CATTCCTGGTTGTCGCGTGT & 822 & (Cejas et \\
\hline sugE-R & $\mathrm{R}$ & GCCTGATATGTCCTGGATCGT & & al., 2014) \\
\hline
\end{tabular}

Abbreviations: F, forward primer; $\mathrm{R}$, reverse primer

Verification of mPCR specificity was first optimized using donor and recipient colony-lysates. A single colony forming unit was transferred to $200 \mu \mathrm{L}$ of Triton X-100 and boiled at $100^{\circ} \mathrm{C}$ for $10 \mathrm{~min}$. The $25 \mu \mathrm{L}$ mPCR reaction mixtures contained $2 \mu \mathrm{L}$ of colony-lysate, $12.5 \mu \mathrm{L}$ of $2 \mathrm{X}$ TopTaq Mastermix (Qiagen, USA), $2 \mathrm{mM} \mathrm{MgCl} 2,0.2 \mu \mathrm{M}$ of $b l a_{\mathrm{CMY}-2}$ primers, $0.3 \mu \mathrm{M}$ of isolate specific primers, and $0.3 \mu \mathrm{M}$ of $b l c-F$ and $s u g E-R$ primers (Table 5.4). PCR was performed using a BioRad C1000 Touch Thermal Cycler (BioRad, CA, USA) with the following conditions: 5 min at $94^{\circ} \mathrm{C}, 34$ cycles of $30 \mathrm{~s}$ denaturation at $94^{\circ} \mathrm{C}, 30 \mathrm{~s}$ annealing at $61^{\circ} \mathrm{C}$, and $60 \mathrm{~s}$ elongation at $72^{\circ} \mathrm{C}$, followed by a final elongation at $72^{\circ} \mathrm{C}$ for $10 \mathrm{~min}$. 


\subsubsection{Mating experiments}

Transfer by conjugation was performed using a Salmonella ser. Heidelberg donor (Table 5.2) and each recipient (Table 5.3). Donors and recipients were grown separately in $5 \mathrm{~mL} \mathrm{LB}$ broth containing $4 \mu \mathrm{g} / \mathrm{mL}$ CTX (donor) or $100 \mu \mathrm{g} / \mathrm{mL}$ Rif (recipients) at $37^{\circ} \mathrm{C}$ with shaking at $100 \mathrm{rpm}$ for $20 \mathrm{hrs}$. Cultures of donor and recipient were diluted to an optical density of 1.0, and cells from $1.6 \mathrm{~mL}$ were pelleted by centrifugation for $2 \mathrm{~min}$. at $8,600 \mathrm{rpm}$. The supernatant was discarded, and the pellet was resuspended in $800 \mu \mathrm{L}$ SPBS. Resuspensions of donor and recipient were then mixed 1:1 and $100 \mu \mathrm{L}$ of the mixture was plated on both LBA and M9A in duplicate. Conjugation mixtures on both agars were incubated for $2 \mathrm{hrs}$ at $37^{\circ} \mathrm{C}$ or $25^{\circ} \mathrm{C}$ unless otherwise stated. Following $2 \mathrm{hrs}$ incubation, the conjugation mixture was scraped from the plate using $1 \mathrm{~mL}$ SPBS. Suspensions were serially diluted and plated on UChrom supplemented with $100 \mu \mathrm{g} / \mathrm{mL}$ Rif and 1 (CTX1) or 4 (CTX4) $\mu \mathrm{g} / \mathrm{mL}$ of CTX to select for transconjugants. Dilutions were also drop plated on UChrom containing $100 \mu \mathrm{g} / \mathrm{mL}$ Rif to enumerate CFU/mL of recipients. Conjugation frequencies were calculated as transconjugants per recipient.

\subsubsection{Verification of recipients}

Transconjugants obtained after mating were confirmed by both colony morphology on UChrom and mPCR. With the exception of Proteus sp., recipient colonies appeared either blue or purple on UChrom while the Salmonella donor grew as large white colonies. For each transconjugant plate, 1-10 CFU with colony morphology matching the recipient were analysed by mPCR (Table 5.4). Single transconjugant colonies were selected with a sterile pick. A short streak of the picked colony was 
transferred to a gridded UChrom plate, then transferred to a $25 \mu \mathrm{L}$ mPCR reaction mixture. One colony of recipient and donor strains were included as negative and positive controls. mPCR reactions were carried out as described above. Amplicons were analysed using capillary electrophoresis on the QIAxcel DNA Screening system using method AM420 with QX alignment markers 15/3000 bp and QX DNA size marker $100-2500$ bp. Successful transconjugants produced amplicons for both $b l a_{\mathrm{CMY}-2}$ and $b l c-s u g E$ but not OLC2644 (Table 5.4). For each strain tested, one recipient from successful matings (mPCR confirmed) was cultured from gridded UChrom plate in LB + Rif $+\mathrm{CTX}$ at $37^{\circ} \mathrm{C}$ overnight, centrifuged at 8,600 rpm and resuspended in fresh LB without antibiotics, then mixed with an equal volume of 30\% glycerol (Fisher Scientific: Alfa Aesar, Ottawa, Canada) for long term storage.

\subsubsection{Plasmid Stability}

Methods were adapted from Porse et al. (2016) and Harrison et al. (2015). Triplicates of each plasmid-carrying strain were passaged once daily, for 14 days, in 96well plates containing $150 \mu \mathrm{L}$ LB-Lennox broth to assess plasmid stability (approximately 101 generations). Each day $1 \mu \mathrm{L}$ of culture was transferred to the corresponding well of a new plate using a 48-pin replicator. Plates were incubated at $37^{\circ} \mathrm{C}$ with shaking at 100 rpm. Following transfer, 30\% glycerol was added to each well and plates were frozen for future processing.

To determine plasmid stability, plates were thawed and a $20 \mu \mathrm{L}$ aliquot of each culture was removed and serially diluted in SPBS. Plates containing remaining culture were stored at $-80^{\circ} \mathrm{C}$ for future analyses. Serial dilutions were drop-plated in $5 \mu \mathrm{L}$ 
aliquots on UChrom, UChrom + CTX1, and UChrom + CTX4. Agar drop-plates were incubated at room temperature overnight. Where colonies were too small to count, dropplates were placed in $37^{\circ} \mathrm{C}$ incubator and monitored until countable colony sizes were achieved. Cell concentration $(\mathrm{CFU} / \mathrm{mL})$ was determined for all cultures. To calculate the rate of plasmid retention, colonies from drop plates without CTX were analysed using mPCR (described above). Plasmid loss was measured as negative mPCR result(s).

\subsubsection{Phylogenetic tree construction from bacterial core genomes}

Phylogenetic tree construction for core genome comparison of donor and recipient strains was conducted using bcgTree (Ankenbrand and Keller, 2016). Briefly, WGS assemblies for donor and recipients were annotated using Prokka (Seemann, 2014), and protein fasta output files were utilized by bcgTree. Conjugation frequency data was integrated with the bcgTree phylogeny using the ggtree package (Yu et al., 2017) in R version 4.0.2 (R Core Team, 2014). Phylogenetic distances between isolates were calculated from the bcgTree output using the ape package (Paradis and Schliep, 2019) in $\mathrm{R}$ version 4.0.2 (R Core Team, 2014).

\subsubsection{Statistical Analyses}

Significant effects of temperature and nutrient (agar type) conditions on mating were investigated for each isolate using one-way analysis of variance (ANOVA) on $\log _{10^{-}}$ transformed data, followed by Tukey's multiple pairwise-comparisons. One-way ANOVAs were also conducted to investigate differences between isolates for each condition (M9 $25^{\circ} \mathrm{C}, \mathrm{M} 937^{\circ} \mathrm{C}, \mathrm{LB} 25^{\circ} \mathrm{C}$, and $\mathrm{LB} 37^{\circ} \mathrm{C}$ ), and to investigate differences 
between plating on CTX1 versus CTX4 for each isolate. All analyses were conducted using R version 4.0.2 (R Core Team, 2014).

\subsection{Results}

No transconjugants were observed for conjugation experiments with any of the Proteus mirabilis recipients in this study at 2 nor 4-hour conjugation times. Longer mating times were not attempted, nor were lower concentrations of CTX for selection.

Following conjugation with Hafnia alvei strains Haf1276, Haf1306, and Haf3292, clusters of very small colonies were observed on UChrom + Rif100 + CTX1 plates in addition to larger colonies (data not shown). However, this was not the case for Hafl 276 where the donor specific (OLC2644) amplicon was also observed, nor Haf1306 where amplicons for the $b l a_{\mathrm{CMY}-2}$ and adjacent $\operatorname{sug} E$ and $b l c$ genes were not observed in transconjugants. As no isolates with PCR-confirmed plasmids were retained, Hafl276, Haf1306, and Haf3293 were not included in further experiments.

All remaining recipient strains successfully conjugated with the donor Salmonella ser. Heidelberg, resulting in transfer of the IncI plasmid encoding the bla $a_{\mathrm{CMY}-2}-b l c-s u g E$ cassette (Figure 5.1). Of the recipients investigated, a range of transfer frequencies from low $\left(10^{-9} \mathrm{CFU}\right.$ transconjugants / CFU recipient $)$ to high $\left(10^{-2} \mathrm{CFU}\right.$ transconjugants / CFU recipient) were observed (Figure 5.1). Although transconjugants were observed for most strains under all nutrient-temperature conditions in at least one of 3 to 4 independent experiments, plasmid transfer was not observed following mating on M9A at $25^{\circ} \mathrm{C}$ for Klebsiella strains Klb1672 and Klb2894 (Figure 5.1). In addition, the conjugation rate was significantly higher when enumerated on agar containing lower concentrations of 
CTX (CTX1 versus CTX4) for Cit2431 and Klb1227. Within genera there were differences in conjugation rates between strains (Figure 5.1, Figure 5.2). Between genera, E. coli K-12 strain MG1655 exhibited highest conjugation rate followed by Enterobacter Ent2920, and Citrobacter Cit1224 (Figure 5.1, Figure 5.2).

Citrobacter isolate Cit2431 only produced a single transconjugant in one experiment following mating on both M9A and LBA at room temperature (Figure 5.1). Klebsiella isolate Klb2894 only produced transconjugants following incubation on LBA at $37^{\circ} \mathrm{C}$ (Figure 5.1). Longer conjugation times were not attempted for these isolates in this study. 
Figure 5.1. Foodborne bacteria readily conjugate with a foodborne pathogen under different nutrient-temperature conditions. (Page 153) Conjugation frequency (obtained transconjugants/recipient CFU) between donor $S$. Heidelberg and foodborne isolates (recipients, panel labels) was determined under different nutrient (LBA - nutrient rich, M9A - minimal nutrients) and temperature $\left(37^{\circ} \mathrm{C}\right.$ and $\left.25^{\circ} \mathrm{C}\right)$ conditions (x-axis). Following 2-hour solid-phase matings, conjugation mixtures were enumerated on UChrom agar containing 1 or $4 \mu \mathrm{g} / \mathrm{mL}$ cefotaxime (CTX). Box and whisker plots show the minimum, maximum, and median of 3 to 4 independent mating experiments for each strain. Outliers are denoted by small solid circles above or below box. Strains are displayed in separate panels. One-way analysis of variance (ANOVA) of conjugation frequency with Tukey's multiple comparisons was implemented to determine statistical significance between conjugation conditions for enumerations on $1 \mu \mathrm{g} / \mathrm{mL} \mathrm{CTX}$ (grey solid bars), or $4 \mu \mathrm{g} / \mathrm{mL}$ CTX (black dashed bars): $p<0.05=* ; p<0.01=* * ; p<0.001$ $=* * *$. 


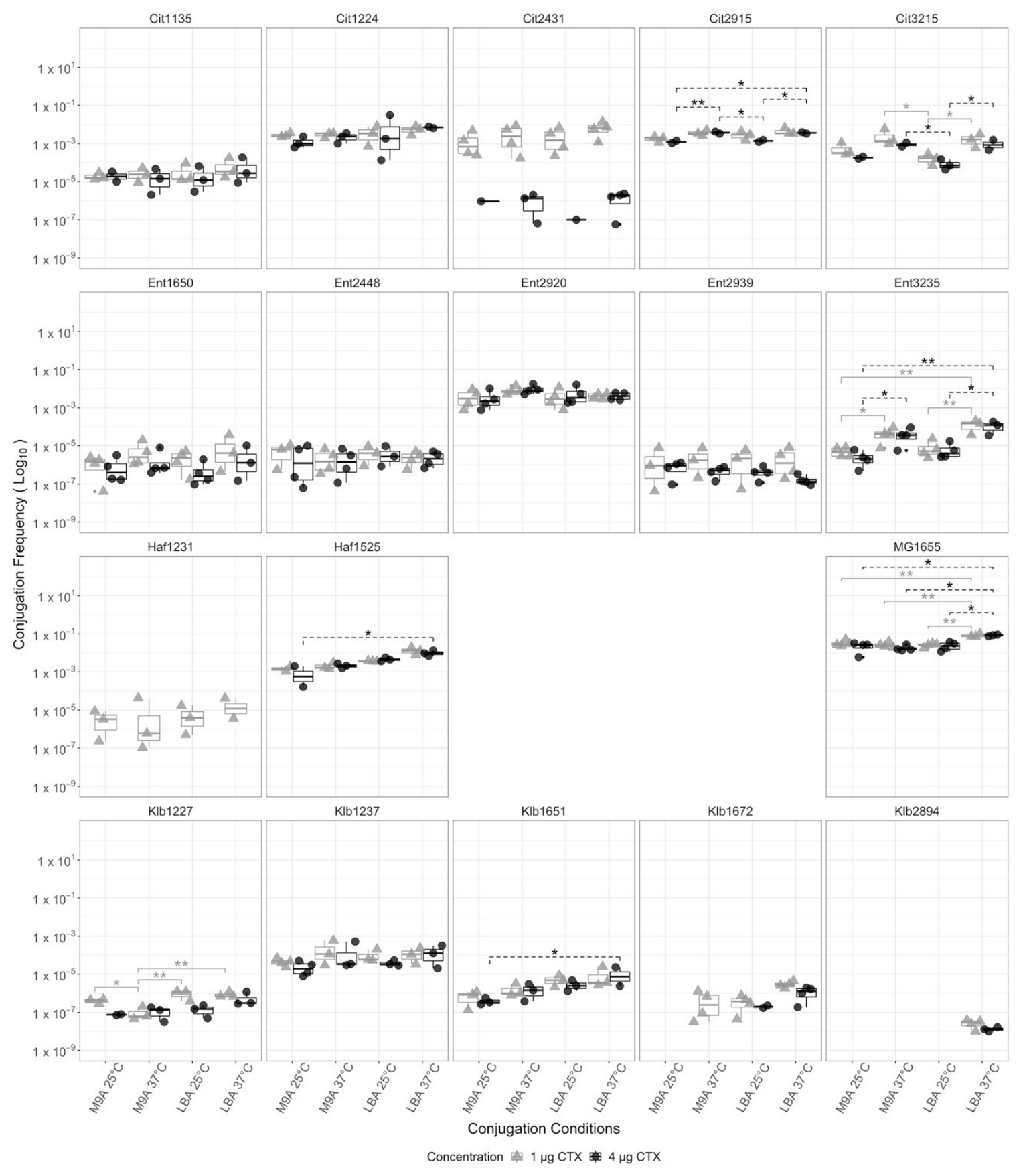




\subsubsection{MICs of recipients and transconjugants}

Prior to conjugation experiments, all but one recipient exhibited an MIC of 0.5 $\mu \mathrm{g} / \mathrm{mL}$ cefotaxime on both MHA, UChrom and LBA (Table 5.5). Proteus mirabilis isolates from the OLC strain collection harbouring bla $a_{\mathrm{CMY}-2}$ had MIC values of $2 \mu \mathrm{g} / \mathrm{mL}$ CTX which were lower than the bla $a_{\mathrm{CMY}-2}$ donor Salmonella strain, therefore all mating mixtures were plated on both 1 and $4 \mu \mathrm{g} / \mathrm{mL}$ CTX following $2 \mathrm{hrs}$ conjugation (data not shown).

Successful transconjugants' MICs ranged from 4 to $\geq 8 \mu \mathrm{g} / \mathrm{mL}$ on MHA, UChrom and LBA for all strains except Haf1231 which exhibited an MIC of $2 \mu \mathrm{g} / \mathrm{mL}$ on MHA and LBA, and $4 \mu \mathrm{g} / \mathrm{mL}$ on UChrom (Table 5.5). 
Table 5.5. Minimum inhibitory concentrations (MICs) of cefotaxime (CTX) for recipients and transconjugants on MHA, UChrom, and LBA-Lennox

$\operatorname{MIC}(\mu \mathrm{g} / \mathrm{mL})^{\mathrm{a}}$

\begin{tabular}{|c|c|c|c|c|c|c|}
\hline \multirow[t]{2}{*}{ ID } & \multicolumn{3}{|c|}{ Recipient } & \multicolumn{3}{|c|}{ Transconjugant } \\
\hline & MHA & UChrom & LBA & MHA & UChrom & LBA \\
\hline Cit1135 & 0.5 & 0.5 & 0.5 & $>8$ & $>8$ & $>8$ \\
\hline Cit1224 & 0.5 & 0.5 & 0.5 & 4 & $>8$ & 8 \\
\hline Cit2431 & 0.5 & 0.5 & 0.5 & 4 & $>8$ & 8 \\
\hline Cit2915 & 0.5 & 0.5 & 0.5 & $>8$ & $>8$ & $>8$ \\
\hline Cit3215 & 0.5 & 0.5 & 0.5 & 4 & $>8$ & 8 \\
\hline Ent1650 & 0.5 & 0.5 & 0.5 & 8 & $>8$ & 8 \\
\hline Ent2448 & 0.5 & 0.5 & 0.5 & $>8$ & $>8$ & $>8$ \\
\hline Ent2920 & 0.5 & 0.5 & 0.5 & $>8$ & $>8$ & $>8$ \\
\hline Ent2939 & 0.5 & 0.5 & 0.5 & 8 & $>8$ & 8 \\
\hline Ent3235 & 0.5 & 0.5 & 0.5 & 8 & $>8$ & $>8$ \\
\hline Haf1231 & 0.5 & 0.5 & 0.5 & 2 & 4 & 2 \\
\hline Haf1525 & 0.5 & 0.5 & 0.5 & 8 & $>8$ & 8 \\
\hline Klb1227 & 0.5 & 0.5 & 0.5 & 4 & 8 & 4 \\
\hline Klb1237 & 0.5 & 0.5 & 0.5 & 4 & 8 & 8 \\
\hline Klb1651 & 0.5 & 0.5 & 0.5 & 4 & 8 & 4 \\
\hline Klb1672 & 0.5 & 0.5 & 0.5 & 8 & 8 & 8 \\
\hline Klb2894 & 0.5 & 0.5 & 0.5 & 4 & 8 & 8 \\
\hline MG1655 & 0.5 & 0.5 & 0.5 & $>8$ & $>8$ & $>8$ \\
\hline Donor & $>8$ & $>8$ & $>8$ & NA & NA & NA \\
\hline
\end{tabular}

Abbreviations: MIC, minimum inhibitory concentration; MHA, Mueller-Hinton agar; UChrom, Universal Differential Chromoselective medium (Millipore-Sigma, Canada); LBA, Lysogeny broth agar - Lennox formulation; NA, not applicable.

${ }^{\mathrm{a}} \mathrm{MIC}$ was defined as the lowest concentration of cefotaxime to completely inhibit growth. 


\subsubsection{Impact of CTX concentration on enumeration of transconjugants}

Significantly higher rates of conjugation were observed for some strains when transconjugants were enumerated using the lower concentration of CTX1 for selection. Conjugation frequency was significantly higher when enumerated on agar containing CTX1 versus CTX4 for Klb1227 on LBA at $25^{\circ} \mathrm{C}(\mathrm{p}<0.047)$; and strain Cit2431 on M9A at $25^{\circ} \mathrm{C}(\mathrm{p}<0.023)$, M9A at $37^{\circ} \mathrm{C}(\mathrm{p}<0.0001), \mathrm{LBA}$ at $25^{\circ} \mathrm{C}(\mathrm{p}<0.0011)$, and LBA at $37^{\circ} \mathrm{C}$ $(\mathrm{p}<0.00001)$. Hafnia alvei isolate Haf1231 only produced transconjugants able to grow on CTX1, not CTX4 (Figure 5.1). Similarly, Klebsiella isolate Klb1672 did not produce transconjugants able to grow on CTX4 following 2 hours incubation on M9A at either temperature $\left(25^{\circ} \mathrm{C}\right.$ and $\left.37^{\circ} \mathrm{C}\right)$, nor transconjugants able to grow on CTX1 after mating on M9A at $25^{\circ} \mathrm{C}$ (Figure 5.1).

\subsubsection{Impact of Nutrients and temperature on conjugation frequency}

A small number of significant differences between plasmid transfer frequencies under tested conditions (M9A $25^{\circ} \mathrm{C}$, M9A $37^{\circ} \mathrm{C}$, LBA $25^{\circ} \mathrm{C}$, and $\mathrm{LBA} 37^{\circ} \mathrm{C}$ ) were observed for seven isolates (Figure 5.1). In four cases conjugation frequencies were higher at $37^{\circ} \mathrm{C}$ than at $25^{\circ} \mathrm{C}$. For Cit2915 conjugation frequencies enumerated on CTX4 were significantly higher at $37^{\circ} \mathrm{C}$ compared to $25^{\circ} \mathrm{C}$ for both M9A and LBA (Figure 5.1). Conjugation frequencies were significantly lower on $\mathrm{LBA}$ at $25^{\circ} \mathrm{C}$ compared to both M9A and LBA at $37^{\circ} \mathrm{C}$ in Cit3215. Conjugation rates were also significantly higher at $37^{\circ} \mathrm{C}$ versus $25^{\circ} \mathrm{C}$ for Ent3235 on both M9A and LBA (Figure 5.1). Similarly, 
conjugation rates in MG1655 were significantly higher at $37^{\circ} \mathrm{C}$ on LBA versus all other conditions for enumerations on both CTX1 and CTX4 (Figure 5.1).

\subsubsection{Between strains comparison of conjugation frequency}

Analysis of variance found many significant differences in conjugation rates between strains for all tested conditions (Figure 5.2). Transfer of the plasmid to E. coli K12 control strain MG1655 occurred at a significantly higher rate than all other recipients except for Citrobacter strains Cit1224 and Cit2915, and Hafnia strain Haf1525, under all tested conditions. Similarly, transfer rates to Enterobacter Ent2920 were only significantly different than those of recipient MG1655 on LBA at $37^{\circ} \mathrm{C}$ when enumerated on UChrom + CTX1 $(p=0.0311)$. 
Figure 5.2. Conjugation frequencies are significantly different between strains of foodborne bacteria. (Page 159) One-way analysis of variance (ANOVA) of conjugation frequency with Tukey's multiple comparison tests was implemented to determine statistical significance between strains under each condition (LBA $37^{\circ} \mathrm{C}$, LBA $25^{\circ} \mathrm{C}$, M9A $37^{\circ} \mathrm{C}, \mathrm{M} 9 \mathrm{~A} 25^{\circ} \mathrm{C}$ ) for enumerations on $1 \mu \mathrm{g} / \mathrm{mL} \mathrm{CTX}$ (top row), or 4 $\mu \mathrm{g} / \mathrm{mL}$ CTX (bottom row). Intersect between strain on y-axis and $\mathrm{x}$-axis denotes significance: $p<0.05=* ; p<0.01=* * ; p<0.001=* * * ; p<0.0001=* * * *$. Intensity of red fill reflects significance value: $p=1$, white; $p=1 \times 10^{-20}$, bright red. Missing box(es) indicate no transconjugants were obtained under corresponding conjugation condition(s). 
LBA $37^{\circ} \mathrm{C}$

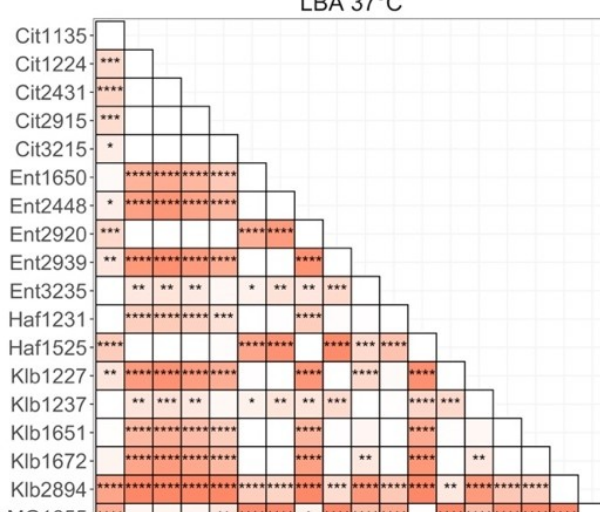

Klb2894:.....

Cit1135- $\square$

Cit1224....

Cit2431 - . - ....

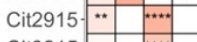

Cit3215.

Ent1650.

Ent2920-...

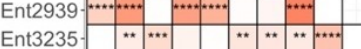

Haf1231

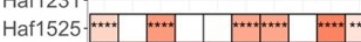

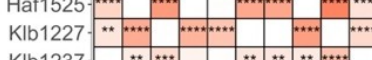

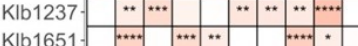

Klb1672:-.....

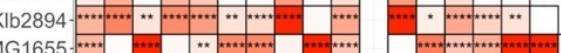

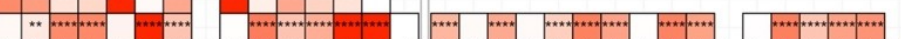

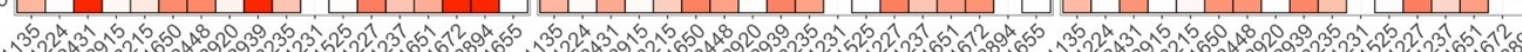
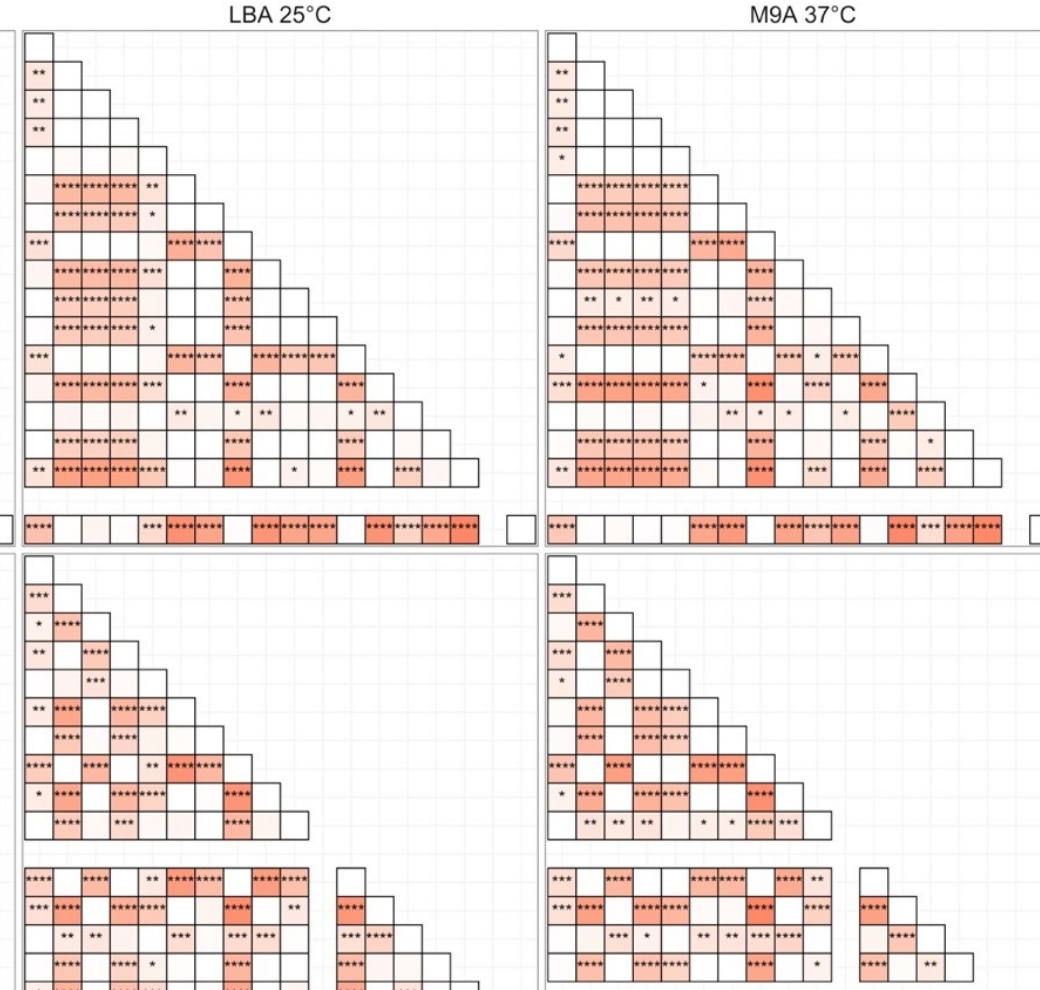

$\mathrm{M} 9 \mathrm{~A} 25^{\circ} \mathrm{C}$

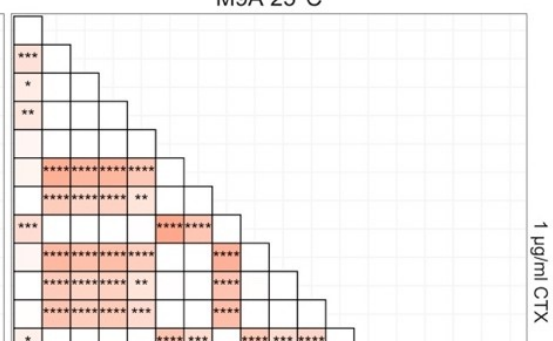

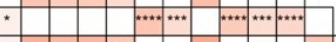

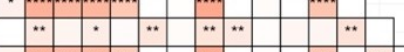

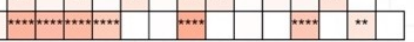
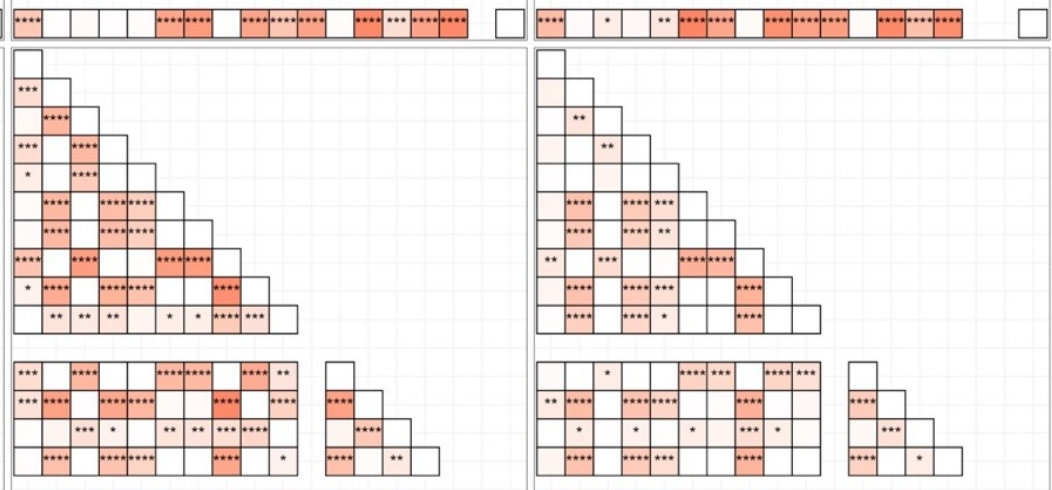


\subsubsection{Transfer rate as a function of donor-recipient core-genome relatedness}

Pairwise phylogenetic distance analysis of core genomes using bcgTree and $\mathrm{R}$ determined the Escherichia coli K-12 lab strain MG1655 to be the most closely related isolate to the $S$. ser. Heidelberg donor, followed by Citrobacter, Enterobacter, Klebsiella, Hafnia, then Proteus genera isolates (Appendix E.1 - Table 5.6, Figure 5.3). Initial comparison of conjugation frequency and phylogeny suggested a possible pattern where transfer rate increased with genetic relatedness (Figure 5.3). Plotting transfer frequency as a function of phylogenetic pairwise distance did not reveal a clear relationship between core-genome relatedness and transfer rate for the plasmid in this study (Figure 5.4).

\subsubsection{Plasmid stability in different genera}

The plasmid was stably maintained in the donor Salmonella and all tested transconjugants, in all replicates, for approximately 100 generations (14 passages) without selective pressure (Figure 5.5). Transconjugants Ent1650, E. coli MG1655, Klb1227, Klb1237, Klb1672, and Klb2894 produced extremely small colonies on UChrom compared to other isolates following passaging. 


\section{Genus 追 Citrobacter 追 Enterobacter 追 Escherichia 追 Hafnia 追 Klebsiella}
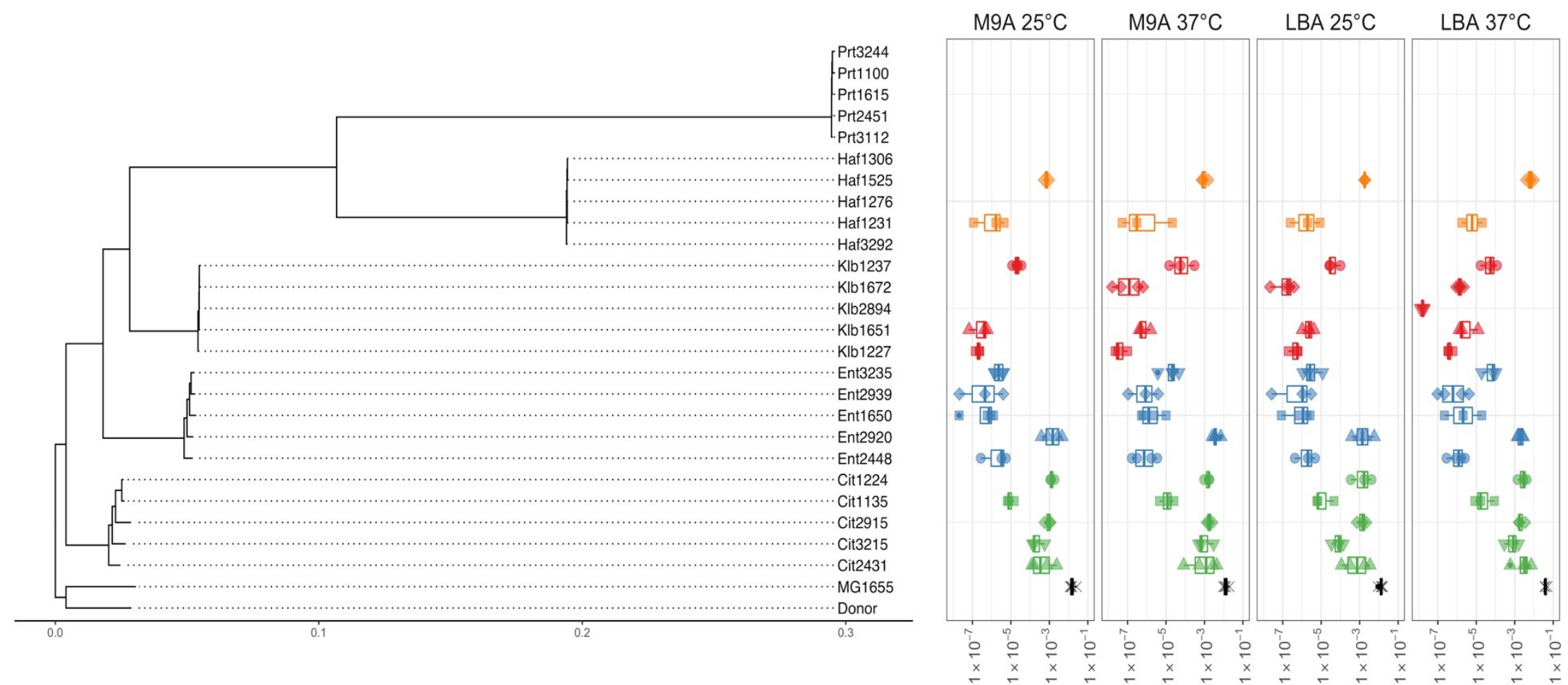

Figure 5.3. Rate of plasmid transfer may be higher for more closely related recipient strains. Phylogenetic tree was constructed from isolate WGS data (core genomes) using bcgTree. Boxplots of conjugation frequency (obtained transconjugants/recipient CFU, $\mathrm{x}$-axis) between donor Salmonella ser. Heidelberg and foodborne isolates (recipients, tree tips/y-axis) was determined under different nutrient and temperature (LBA or $\mathrm{M} 9 \mathrm{~A}, 37^{\circ} \mathrm{C}$ and $25^{\circ} \mathrm{C}$ ) conditions (panel headings). Following 2-hour solid-phase matings, conjugation mixtures were enumerated on Universal Differential Chromoselect Medium (UChrom) agar (Millipore Sigma, Canada) containing $1 \mu \mathrm{g} / \mathrm{mL}$ cefotaxime (CTX). Boxplots and data points correspond to strain of adjacent tree tip. Results from enumeration on UChrom containing CTX1 are displayed, data for $4 \mu \mathrm{g} / \mathrm{mL}$ CTX not shown. 


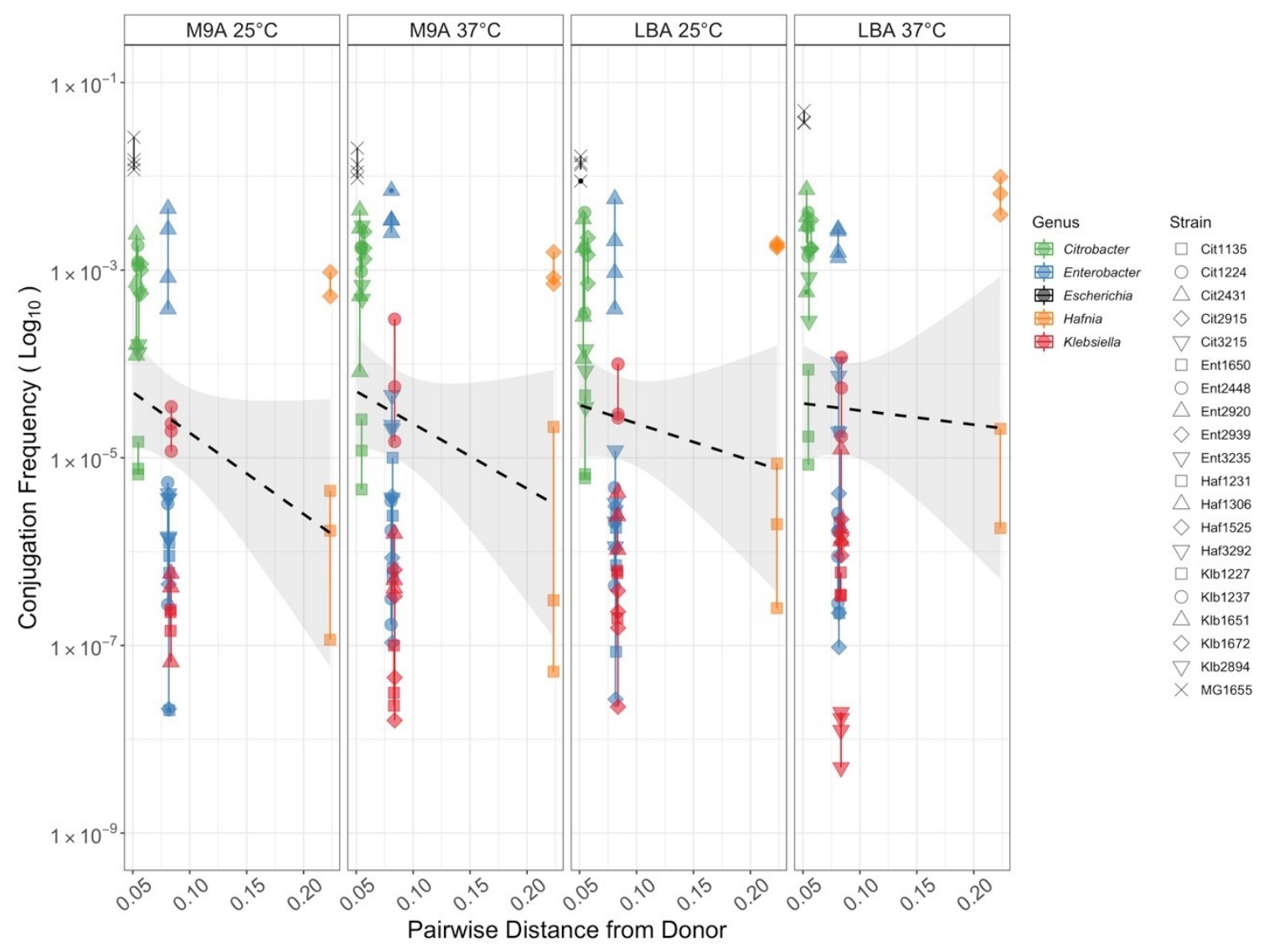

Figure 5.4. Conjugation frequency as a function of donor-recipient relatedness.

Phylogeny was constructed from isolate WGS data (core genomes) using bcgTree, then pairwise distance from donor strain was calculated using the cophenetic.phylo function from the ape package in R statistical software v. 4.0.2 from the bcgTree output. Conjugation frequency (obtained transconjugants/recipient, CFU/mL, y-axis) between donor Salmonella ser. Heidelberg and foodborne isolates (recipient strains) was determined under different nutrient and temperature (LBA or $\mathrm{M} 9 \mathrm{~A}, 37^{\circ} \mathrm{C}$ and $25^{\circ} \mathrm{C}$ ) conditions (panel headings). Following 2-hour solid-phase matings, conjugation mixtures were enumerated on Universal Differential Chromoselect Medium (UChrom) agar (Millipore Sigma, Canada) containing $1 \mu \mathrm{g} / \mathrm{mL}$ cefotaxime (CTX). Recipient genera are differentiated by colour, strains are differentiated by point shape. Results from enumeration on UChrom containing CTX1 are displayed, data for $4 \mu \mathrm{g} / \mathrm{mL} \mathrm{CTX} \mathrm{not}$ shown. Fitted line (dashed) is a linear model. 


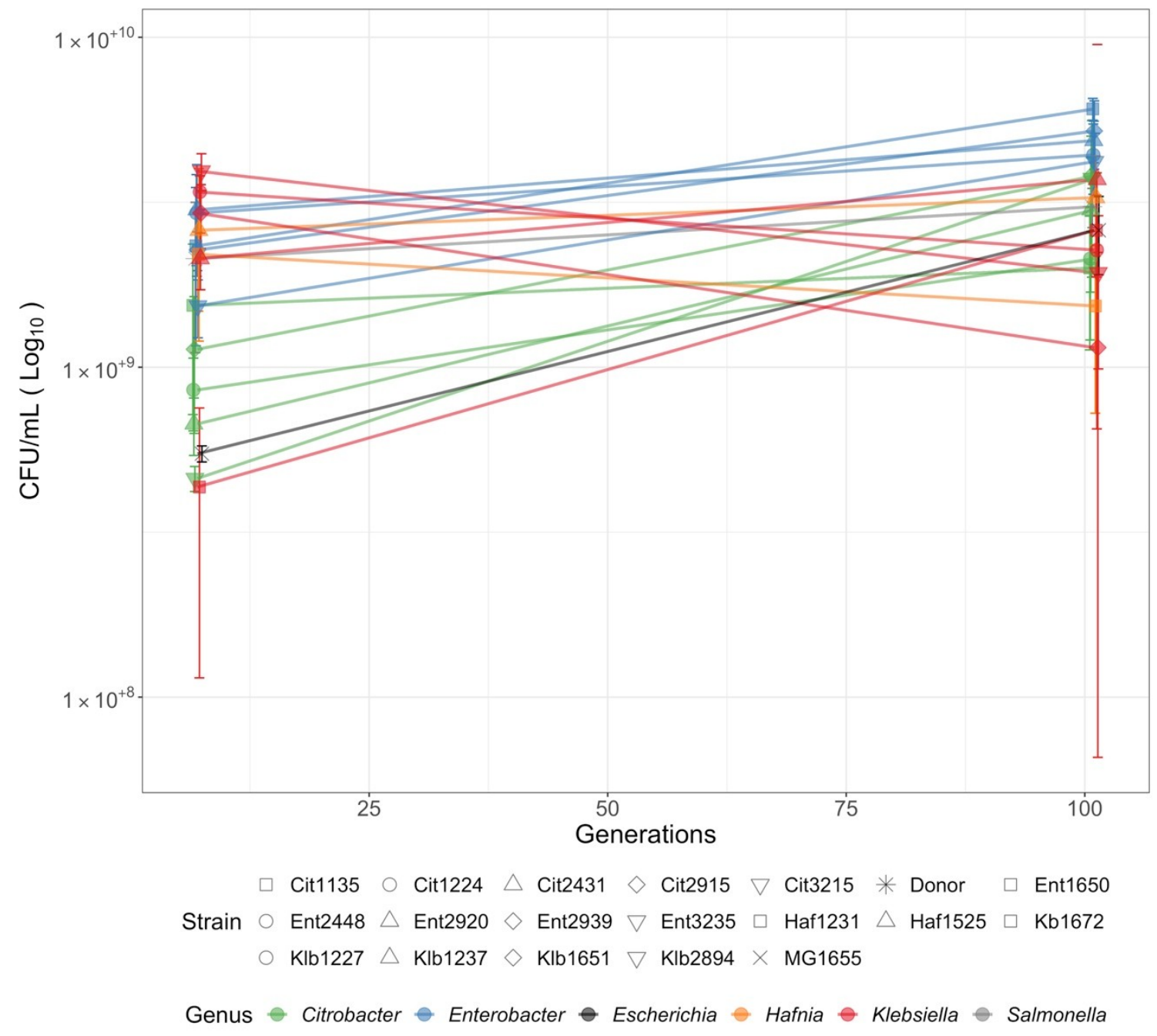

Figure 5.5. Foodborne bacteria stably maintain plasmid encoding blaCMY-2 for

100 generations. Triplicates of donor (Salmonella) and transconjugant strains harbouring the $b l a_{\mathrm{CMY}-2}$ plasmid were passaged daily in $150 \mu \mathrm{L}$ of Lysogeny BrothLennox for 14 days (1:150, approximately 101 generations). Transformed ( $\log 10)$ counts of colony forming units $(\mathrm{CFU} / \mathrm{mL})$ (y-axis) are plotted as a function of bacterial generations (x-axis). Averages of three technical replicates +/- standard deviation are displayed for each strain. Genera are differentiated by colour, and strains are differentiated by point shape. 


\subsection{Discussion}

Transfer of AMR-encoding plasmids plays a role in clinical infections where acquisition of ARGs may assist in infection (Dimitriu et al., 2019). E. coli and Salmonella isolates are documented to carry transposon-associated $b l a_{\mathrm{CMY}-2}$ which can be transferred between bacterial species as well as transmitted from food animals to humans (Carattoli et al., 2002; Chiu et al., 2020). This study investigated the frequency of transfer of a conjugative IncI plasmid, measured as the ratio of transconjugants/recipient CFUs, from a strain of the foodborne pathogen Salmonella ser. Heidelberg to 25 strains of common foodborne bacteria from 5 different genera. We found most of the investigated foodborne Enterobacteriaceae strains could readily receive the $b l a_{\mathrm{CMY}-2}$ encoding IncI plasmid, in some cases at high rates comparable to that of the laboratory $E$. coli K-12 commonly utilized in plasmid transfer studies. Varying environmental conditions, including nutrient availability and temperature, had minimal effects on plasmid transfer. However, this was strain dependent (Figure 5.1).

\subsubsection{Production of various morphotypes by Hafnia alvei recipients}

Although antimicrobial susceptibility testing found all potential recipient isolates to be sensitive to cefotaxime, three of the Hafnia alvei isolates in this study (Hafl276, Haf1306, and Haf3292) produced inconclusive results where transconjugants could not be enumerated due to presence of many small colony variants surrounding larger colonies. Strain(s) of $H$. alvei have been documented to produce two morphotypes, a large colony morphotype with low-level and inducible cephalosporinase production and 
susceptible to ceftazidime, and a smaller colony morphotype with higher level cephalosporinase production and resistance to $\beta$-lactam antibiotics (Thomson et al., 1993; Girlich et al., 2000). Isolates Haf1276, Haf1306, and Haf3292 which exhibited growth of small colony morphotypes all encoded bla $_{\mathrm{ACC}-1 \mathrm{a}}($ Table 5.3), which correlates with production of a small colony variant and increased $\beta$-lactam resistance as found in other studies (Girlich et al., 2000).

\subsubsection{Environmental conditions had minimal effect on conjugation frequency to foodborne bacterial strains}

Hardiman et al. (2016) reported plasmid transfer frequency was influenced by both temperature and substrate in addition to donor-recipient combination, and Aviv et al. (2016) found transfer frequency was increased in conditions mimicking the mammalian gut, including at $100 \mathrm{mM} \mathrm{NaCl}$, microaerobic environment, and physiological temperatures. In contrast to their results, we did not find significant differences in transfer frequency under the different nutrient conditions and temperatures tested for most strains (Figure 5.1). However, we only tested a single plasmid and four different environments. Presence of a plasmid-mediated tnpA-bla $\mathrm{CMY}-2_{-2} b l c-s u g E$ has been documented in Salmonella and Shigella (Cejas et al., 2014; Chiu et al., 2020). The plasmid investigated in this study also encoded the $b l a_{\mathrm{CMY}-2}-b l c-s u g E$ cassette. An outer membrane lipoprotein is encoded by blc is expressed under stressful conditions including starvation and high osmolarity (Campanacci et al., 2006), while $\operatorname{sugE}$ a small multidrug resistance efflux gene that can confer resistance to quaternary ammonium compounds (QACs) (Chung and Saier, 2002; Cejas et al., 2014). Sub-inhibitory concentrations of antibiotics have been 
shown to select for cells harboring resistance plasmids (Bottery et al., 2016; Westhoff et al., 2017; Cairns et al., 2018). Similarly, use of biocides and preservatives may also alter the bacterial resistome and select for multidrug resistance in bacteria (Webber et al., 2015; Monte et al., 2019; Oniciuc et al., 2019), and could increase transfer efficiency and plasmid stability (Seier-Petersen et al., 2014; Jutkina et al., 2018). Of the limited investigations of biocide presence on plasmid transfer, some reported reduced plasmid transfer (Al-Masaudi et al., 1991; Pearce et al., 1999), however this appears to be dependent on the biocide (Pearce et al., 1999).

\subsubsection{Transfer to related genera}

There are costs and benefits of transfer to both the donor and recipient hosts (Dimitriu et al., 2016; Carroll and Wong, 2018). Biased transfer between donors and recipients of the same genotype has been observed, which results in selection and favouring of kin (Cairns et al., 2018; Dimitriu et al., 2019). In fact, Dimitriu et al. (2016) suggested that altruistic donation of MGEs occurs when transfer is biased towards cells that share donation alleles (i.e. are related). They have shown that HGT can be promoted by relatedness of bacterial hosts, and is not necessarily dependent on MGEs (Dimitriu et al., 2016).

Klebsiella spp. have been recommended as possible AMR indicators in environmental settings as they are often indicators of new ARG emergence (Berendonk et al., 2015; Navon-Venezia et al., 2017), and Klebsiella pneumoniae has been suggested as a trafficker of drug resistance genes from environmental to clinically important bacteria (Wyres and Holt, 2018). Moreover, as Klebsiella is a genus belonging to the 
Enterobacteriaceae family and is related to Salmonella, we expected to observe high plasmid transfer rates to Klebsiella recipients in this study. The Klebsiella pneumoniae recipient strains in this study all successfully conjugated with the Salmonella donor, however rates of transfer were still significantly lower than observed for the more distantly related Hafnia Haf1525 strain under most tested conditions (Figure 5.2, Figure 5.3). Though all recipient strains were screened for plasmid content prior to experimentation, each Klebsiella recipient still encoded at least one plasmid (Table 5.3). However, none of the incompatibility groups of selected Klebsiella recipients' plasmids were of the same incompatibility type as the donor plasmid (IncI), and evidence from Hardiman et al. (2016) suggests that plasmid incompatibility groups alone may not explain differences in conjugation efficiency.

Conjugation of the IncI plasmid was not observed for Proteus mirabilis recipient strains following 2 nor 4-hour conjugation times, yet plasmid transfer of carbapenemresistance has been observed from $P$. mirabilis to E. coli via conjugation (Hu et al., 2012). Conjugative plasmids can exhibit varying host ranges, with many resistance plasmids known to have broad host ranges (Bennett, 2008; Cairns et al., 2018). Many features may contribute to the host range of plasmids including the structure of and presence of multiple replication origins, genes allowing activation of replication independent of the host, and presence of only a few restriction sites, among others (reviewed in Jain and Srivastava, 2013). The host range of IncI plasmids is predicted to be narrow (Suzuki et al., 2010; Rozwandowicz et al., 2018). Thus, it is possible that the limited host-range of the IncI plasmid investigated in this study, in addition to the more distant relationship between Proteus mirabilis and Salmonella ser. Heidelberg, 
disfavoured conjugal transfer. However, Cairns et al. (2018) did not observe conjugation between E. coli ESBL donors and P. mirabilis recipient-strains either, although the plasmid-characteristics of their donors are not described. Additional investigations with other plasmid types and longer conjugation times should be conducted to further investigate this relationship.

Dimitriu et al. $(2016,2019)$ reported a bias of transfer towards clone-mates that is "not correlated to genetic distance when donors and recipients are not clone-mates". However, our results, together with those of Cairns et al. (2018), suggest relatedness of donor-recipient genera could possibly play a role in conjugation efficiency in (Figure 5.3, Figure 5.4). Although we did observe a slight correlation on M9A at both $25^{\circ} \mathrm{C}$ and $37^{\circ} \mathrm{C}$ where conjugation frequency was higher in isolates more closely related to the donor Salmonella (lower pairwise distance), it is difficult to make definitive statements about the relationship with the small number of strains tested (Figure 5.4). Further studies are needed to investigate additional plasmids, donor-recipient combinations, and phylogenetic analysis of both host and MGE genes.

\subsubsection{Plasmid stability}

Plasmid acquisition can benefit the recipient host via transfer of accessory genes (e.g. ARGs), yet additional genes encoding core plasmid functions can be burdensome thereby decreasing host growth rate and fitness (Turner et al., 1998; Haft et al., 2009; Dimitriu et al., 2016; Carroll and Wong, 2018; Kottara et al., 2020). Research on plasmid persistence in the absence of selection has produced differing results (Dimitriu et al., 2016, 2019; Lopatkin et al., 2017; Gama et al., 2020; Kottara et al., 2020). Although 
plasmids may encode beneficial genes, in theory these genes should integrated into the chromosome and plasmid(s) should be lost (Harrison and Brockhurst, 2012; Harrison et al., 2015; Carroll and Wong, 2018). However plasmid loss does not always occur as expected, thus termed "the plasmid paradox" (reviewed in Harrison and Brockhurst, 2012; Carroll and Wong, 2018). Several theories have been proposed explaining the plasmid paradox including infectious transmission or high-rate conjugative HGT, shuttling of plasmids between bacterial strains and environments, and presence of amplifier strains with high conjugation efficiencies (Harrison and Brockhurst, 2012).

Broad host range plasmids were reported to transfer to a large number recipients belonging to 11 different bacterial phyla in soil (Klümper et al., 2015). However, acquired MGEs may function inefficiently in new genomic backgrounds (Baltrus, 2013). There is evidence that compensatory evolution in both the host and plasmid can reduce the cost of plasmid carriage, thus enabling plasmid persistence (Harrison et al., 2015; Kram et al., 2017). Harrison et al. (2015) found passaging of Pseudomonas fluorescens harbouring a plasmid encoding mercury resistance in different mercury environments resulted in large fitness increases in plasmid-containing strains, but that conjugation rates remained low suggesting plasmids did not persist via conjugation in their experiments. In contrast, Kottara et al. (2020) observed increased transfer of plasmids from Pseudomonas stutzeri to other Pseudomonas species in the presence of mercury.

We investigated the stability of the IncI plasmid in foodborne bacterial strains in rich media without selective pressure. Despite this, all of the transconjugants passaged in this study maintained the plasmid for 100 generations. Additional passaging for more generations, in more limited nutrient conditions, and with addition of a selective pressure 
such as CTX or a QAC, should be conducted to investigate effects on plasmid transfer within foodborne bacteria. Sequencing of evolved transconjugants will determine whether compensatory evolution within the recipient strain and plasmid possibly alleviated the cost of plasmid carriage.

\subsection{Conclusion}

We have confirmed that interspecies transfer of plasmids within the food production environment is possible even in minimal nutrient conditions and at room temperature $\left(25^{\circ} \mathrm{C}\right)$, with plasmid transfer rates varying depending on the strain. The transferred plasmid encoding a $b l a_{\mathrm{CMY}-2}-b l c-s u g E$ resistance cassette was stably maintained in all transconjugant strains for approximately 100 generations in the absence of selection, suggesting related foodborne bacteria could act as a reservoir of clinically relevant AMR in food products. Understanding the importance of the microbiota of foodproducts and environments is critical for accurate risk assessment of AMR in the food production continuum. Further work should be conducted to investigate additional plasmids, the possibility of biocides influencing HGT of ARGs, and the kinetics of transfer from foodborne bacteria to foodborne pathogens. 


\section{Chapter 6: Modeling the Limits of Detection of Antimicrobial \\ Resistance Genes in Agri-Food Metagenomic Samples}

This chapter is an adaptation of a pilot study presented in poster format at the American Society for Microbiology Conference on Rapid Applied Microbial NextGeneration Sequencing and Bioinformatic Pipelines (December, 2020). 


\subsection{Abstract}

Antimicrobial use in medicine and agriculture followed by dissemination into the environment is believed to be one factor which may be contributing to the development of antimicrobial resistance (AMR). Yet the prevalence of AMR organisms (AROs) in agri-food products has not been well characterised. Sequencing technologies are increasingly being used to track the spread of AMR genes (ARGs) in bacteria, and metagenomics has the potential to bypass some of the limitations of single isolate characterization by allowing simultaneous analysis of agri-food product community composition and detection of ARGs. However, metagenomics may still be hindered by methodological biases, presence of eukaryotic DNA, and difficulties detecting low abundance AROs within an attainable sequence coverage. The goal of this pilot study was to assess whether limits of detection of ARGs in agri-food metagenomes were influenced by sample type and bioinformatic approaches. Mock metagenomes were synthesized by simulating five different genome coverage levels $(0.1,1,2,5$, and 10 fold) of Illumina HiSeq short reads from a Salmonella enterica serovar Heidelberg ARO encoding a plasmid-borne $b l a_{\mathrm{CMY}-2}$, then spiking them into a beef fecal metagenomic sequences. Replicate sequences at each coverage level were analysed for ARGs using the SRST2 and KMA tools with the NCBI-AMRFinderPlus database. Accurate detection of ARGs dropped drastically below 5X genome coverage, although it was sometimes possible to detect ARGs and alternative alleles at lower coverage levels if using a lower ARG-target coverage cutoff $(<80 \%)$. While KMA only predicted presence of CMY-2 or CMY-alleles within the same clade, SRST2 falsely reported presence of distantly related ARGs at all isolate genome coverage levels. Overall, ARGs were accurately detected in 
the beef fecal metagenomic sample (approx. 40 million reads) when the ARO constituted a relatively large percentage of the microbiome (e.g. $>0.4 \%$ ). Reducing target identity cutoffs allowed detection of ARGs present at lower abundance. However, this reduced cutoff may result in alternative ARG-allele determination. Further advancements in sequencing technologies facilitating increased depth of coverage, or increased read length, may improve ARG detection in agri-food metagenomic samples enabling use of this approach for tracking low-abundance AROs in agri-food samples.

\subsection{Introduction}

Antimicrobial use in medicine and agriculture are potential drivers of antimicrobial resistance (AMR) dissemination (Berendonk et al., 2015). Many environments including plants, animals, food and water sources can all function as routes for transfer of AMR genes (ARGs) within and between bacterial populations (Huijbers et al., 2015; Bengtsson-Palme, 2017). Food production connects many of these habitats, potentially furthering spread of both AMR and pathogenic bacteria (Bengtsson-Palme, 2017).

Food production occurs along a continuum from production processes to consumption, along which there are multiple points where microbial and crosscontamination may occur. Food-testing practices for detecting bacterial pathogens traditionally requires sampling of food products and production facilities followed by enrichment and culturing for organisms of interest. However, these methods are time consuming, labor intensive, and only target and identify specific pathogenic bacteria (e.g. Salmonella and Listeria monocytogenes). 
A well-known disadvantage of culture-based techniques is that a substantial portion of microorganisms have not yet been cultured (Jernberg et al., 2010). Metagenomic sequencing has the potential to bypass the limitations of culture-based techniques while also enabling evaluation of a sample's microbial diversity (Miller et al., 2013). However, many agri-food sample matrices are complex, containing unknown/unpredictable sample contents and high amounts of eukaryotic DNA.

Many studies have utilized metagenomics to evaluate AMR in various sample matrices (Jernberg et al., 2010; Cantas et al., 2013; Fitzpatrick and Walsh, 2016; Noyes et al., 2016; Thomas et al., 2017). Some research has been conducted investigating the utility of metagenomic sequencing for comparing AMR in different environments (Fitzpatrick and Walsh, 2016). Fitzpatrick and Walsh (2016) observed a bias in the distribution of ARGs where a high abundance was observed in human microbiomes but abundance in marine and soil metagenomes varied greatly in comparison. They concluded that there are limits to detection and identification of ARGs in complex microbiome populations stating that ARGs may not have been detected because they were present below these limits, and that failure to detect ARGs in a metagenome does not equate to absence of ARGs.

Ni et al. (2013) estimated the amount of metagenomic sequencing required to fulfill study objectives. However, they note that prokaryotes encounter different selective pressures in different environments which may affect genome sizes and compositions $(\mathrm{Ni}$ et al., 2013). Luo et al. (2012) found the genome of a single species could be assembled from a metagenome if the species was present with at least 20X coverage (Ni et al., 2013). Similarly, previous work determined ARG detection in isolate WGS data is 
coverage dependent, and that approximately $10 \mathrm{X}$ to $15 \mathrm{X}$ genome coverage is required for accurate detection of ARGs with a target gene identity cutoff of $\geq 90 \%$ (Cooper et al., 2020). Considering shotgun metagenomic sequencing only captures a fraction of the total community within a DNA sample, it is unlikely that all organisms within a sample will be equally abundant at genome coverage above $15 \mathrm{X}$.

The objectives of the current study were to determine the limit of detection (LOD) for ARGs and to compare different in silico bioinformatic tools for their ability to accurately detect ARGs in complex sample matrices, such as those found in agri-food testing.

\subsection{Methods}

\subsubsection{Sequences Used in Mock Metagenome Synthesis}

The whole-genome sequence of a Salmonella enterica serovar Heidelberg encoding $b l a_{\mathrm{CMY}-2}$ was selected from the Ottawa Laboratory Carling Canadian Food Inspection Agency (OLC-CFIA) sequence database. Paired-end raw reads for the $S$. ser. Heidelberg isolate were previously deposited in the Sequence Read Archive (SRA) under accession SRR10859129. Assembly methods for the $S$. ser. Heidelberg sequence were as described previously (Cooper et al., 2020). The metagenomic sequence used as the base for metagenome formulation was a short-read Illumina HiSeq raw-read sequence from a beef fecal sample, published by Noyes et al. (2016) following analysis of cattle resistome diversity. 


\subsubsection{Mock-metagenome Construction}

Illumina HiSeq short reads were synthesized from the draft genome assembly of the $S$. ser. Heidelberg using the FetaGenome2 (fabricate metagenome) tool developed in house (available at: https://github.com/OLC-Bioinformatics/FetaGenome2 ). Briefly, the FetaGenomePlasmidAware edition calculates the depth of each contig in the given assembly using the paired-end raw-read sequence data, then uses the coverage report output to create more reads for higher-depth locations and fewer reads for low-depth locations of the genome. Paired-end HiSeq reads of $150 \mathrm{bp}$ in length with a $300 \mathrm{bp}$ insert size were synthesized to $0.1-, 1-, 2-, 5-$, and 10-X genome coverage of the 4,887,582 bp S. ser. Heidelberg genome (Table 6.1). Ten replicates were created at each coverage level. All replicates were mixed into the HiSeq beef fecal metagenome (SRA accession: SRR3053167) from Noyes et al. (2016), which created a total of 50 synthetic metagenome replicates for analysis. Following concatenation of the replicates into the beef fecal metagenome, all metagenome-replicates were shuffled using fastq-shuffle (available at: https://github.com/chloroExtractorTeam/fastq-shuffle) with the randomseed (-r) setting activated. 
Table 6.1. Characteristics of sequences used for mock-metagenome synthesis

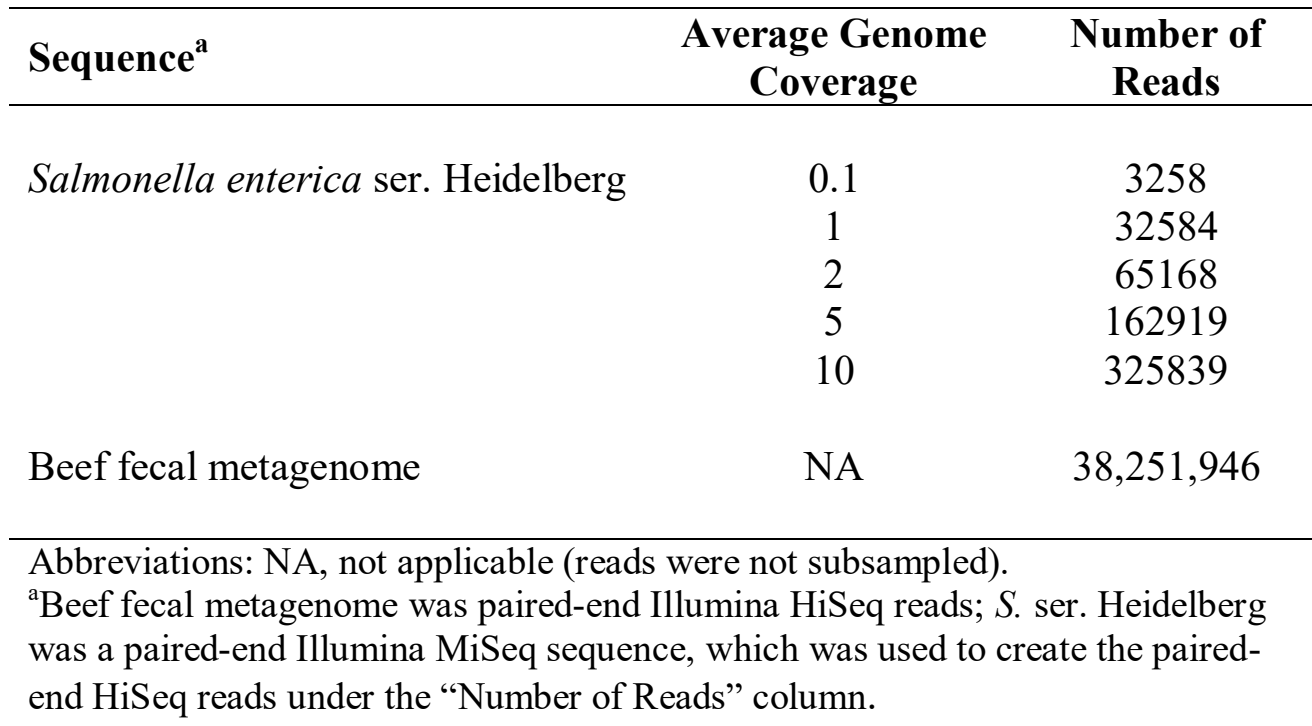

\subsubsection{Metagenome analyses}

For each mock-metagenome replicate, raw-reads were analysed for ARGs using both the $k$-mer alignment (KMA) tool version 1.34 (Clausen et al., 2018) and short read sequence typer version 2 (SRST2) (Inouye et al., 2014) tools with the NCBI AMRFinderPlus Reference Gene Catalog AMR CDS database (downloaded from the NCBI FTP server on 2019-11-01). Default settings were used for detection with KMA. Database clustering for use with SRST2 was conducted according to authors' instructions using Cd-hit (Li and Godzik, 2006) (instructions available at https://github.com/katholt/srst2\#generating-srst2-compatible-clustered-database-fromraw-sequences). For ARG detection with SRST2 minimum coverage was set to 1, and all other settings were left at default. 


\subsubsection{Estimation of reads required for ARG detection}

In order to estimate the ratio of isolate : metagenome reads required for ARG detection at 5- and 10-X isolate coverage a very simple model of "best case scenario", where all reads in a metagenome mapped to bacteria, was used. Estimates for 5- and 10X coverage of 3, 4, and $5 \mathrm{Mbp}$ isolate genomes were calculated and the ratio of each in metagenomes of 5, 10, 40, 50, 100, and $125 \mathrm{Mbp}$ (million base pairs) were determined (Table 6.2).

Table 6.2. Required isolate abundance $(\%)$ in metagenomes of varying sizes for detection of antimicrobial resistance genes in isolates with 3,4 , or $5 \mathrm{Mbp}$ genomes

\begin{tabular}{|c|c|c|c|c|c|c|}
\hline \multirow{3}{*}{$\begin{array}{l}\text { Metagenome } \\
\text { Size }^{\mathrm{a}} \\
\text { (M) }\end{array}$} & \multicolumn{6}{|c|}{ Isolate Percentage in Metagenome ${ }^{b}$} \\
\hline & \multicolumn{2}{|c|}{3 Mbp } & \multicolumn{2}{|c|}{4 Mbp } & \multicolumn{2}{|c|}{5 Mbp } \\
\hline & $\begin{array}{c}5 X \\
(100000)\end{array}$ & $\begin{array}{c}10 \mathrm{X} \\
(200000)\end{array}$ & $\begin{array}{c}5 X \\
(133333)\end{array}$ & $\begin{array}{c}10 X \\
(266666)\end{array}$ & $\begin{array}{c}5 X \\
(166666)\end{array}$ & $\begin{array}{c}10 X \\
(\mathbf{3 3 3 3 3 3})\end{array}$ \\
\hline 5 & 0.08 & 4.00 & 0.11 & 5.33 & 0.13 & 6.67 \\
\hline 10 & 0.10 & 2.00 & 0.13 & 2.67 & 0.17 & 3.33 \\
\hline 40 & 0.20 & 0.50 & 0.27 & 0.67 & 0.33 & 0.83 \\
\hline 50 & 0.25 & 0.40 & 0.33 & 0.53 & 0.42 & 0.67 \\
\hline 100 & 1.00 & 0.20 & 1.33 & 0.27 & 1.67 & 0.33 \\
\hline 125 & 2.00 & 0.16 & 2.67 & 0.21 & 3.33 & 0.27 \\
\hline
\end{tabular}

Abbreviations: M, million; Mbp, million base pairs.

${ }^{\mathrm{a}}$ Metagenome size refers to number of reads in metagenome.

${ }^{b}$ For each genome size $(3,4$, and $5 \mathrm{Mbp}) 5$ - and $10-\mathrm{X}$ genome coverage are estimated (with number of reads to create specified coverage level in parentheses). Percentages are corresponding to metagenome size in first column.

\subsection{Results}

\subsubsection{Coverage of organism affects ARG detection in metagenomes}

The $b l a_{\mathrm{CMY}-2}$ and additional CMY-alleles were not observed in the unspiked (control) beef metagenome (Figure 6.1, 0X panel). Coverage of the $S$. ser. Heidelberg 
isolate in the metagenome affected the proportion out of ten trials that the $b l a_{\mathrm{CMY}-2}$ target gene was accurately detected (Figure 6.1). As genome coverage increased to 10X (Figure 6.1, 10X panel), the target ARG (bla $\left.a_{\mathrm{CMY}-2}\right)$ was reliably detected at $\geq 80 \%$ template coverage in all replicates using both KMA and SRST2. The bla $a_{\mathrm{CMY}-2}$ gene was also detected at $\geq 80 \%$ ARG template coverage in all $5 \mathrm{X}$ replicates using KMA, but only eight out of ten replicates for SRST2 (Figure 6.1).

At lower isolate-genome coverage levels, alleles closely related to the target gene were sometimes detected at a lower template coverage. For example, at $0.1 \mathrm{X}$ coverage, KMA detected the CMY-2 clade bla $a_{\mathrm{CMY}-61}$ allele (99.91\% identity to CMY-2) in one replicate at $40-60 \%$ target template coverage but did not detect any alleles at $\geq 60 \%$ (Figure 6.1).

KMA accurately identified the target gene or closely related alleles even at low ARG target coverage (Figure 6.1). However, SRST2 predicted non-target ARGs at $\geq$ $80 \%$ coverage in some replicates, even when the isolate genome was present in the metagenome at 10X coverage (Figure 6.1). For example, at 1X Salmonella genome coverage KMA detected $b l a_{\mathrm{CMY}-2}$ at $\geq 80 \%$ template coverage in three of ten replicates and related CMY-alleles in the other seven replicates at between 40 and 79\% CMYtemplate coverage, totaling one CMY-gene detected in each of the replicates (at various identities) (Table 6.3, Figure 6.1). In contrast, SRST2 detected $b l a_{\mathrm{CMY}-2}$ at $\geq 80 \%$ in two of ten replicates and eight related CMY-alleles in the other eight replicates, but also detected a number of non-CMY-2 alleles at various coverage levels totaling 43 gene predictions in the ten replicates (Table 6.3, Figure 6.1). 
Table 6.3. Number of CMY-gene(s) and allele(s) detected by KMA and SRST2 in beef metagenomes containing $S$. ser. Heidelberg isolate present at $1 \mathrm{X}$ genome coverage $(\mathrm{n}=10)$

\begin{tabular}{|c|c|c|c|c|c|c|c|c|}
\hline \multirow{3}{*}{$\begin{array}{c}\text { CMY } \\
\text { Template } \\
\text { Coverage }\end{array}$} & \multicolumn{8}{|c|}{ Detected ARG Relatedness CMY-2 } \\
\hline & \multicolumn{2}{|c|}{ CMY-2 } & \multicolumn{2}{|c|}{ CMY-2 clade } & \multicolumn{2}{|c|}{$\begin{array}{c}\text { Other CMY } \\
\text { Alleles }\end{array}$} & \multicolumn{2}{|c|}{ Totals $^{b}$} \\
\hline & KMA & SRST2 & KMA & SRST2 & KMA & SRST2 & KMA & SRST2 \\
\hline$<\mathbf{2 0}$ & - & - & - & - & - & $\begin{array}{c}\text { 20: } \\
4 \mathrm{x} \\
\text { CMY-70, } \\
1 \mathrm{x} \\
\text { CMY-83, } \\
1 \mathrm{x} \\
\text { CMY-100, } \\
6 \mathrm{x} \\
\text { CMY-157, } \\
8 \mathrm{x} \\
\text { CMY- } 159\end{array}$ & - & 20 \\
\hline $20-40$ & - & - & - & $\begin{array}{c}\text { 1: } \\
\text { CMY- } \\
44\end{array}$ & - & $\begin{array}{c}5: \\
\text { CMY-65, } \\
\text { CMY-74, } \\
\text { CMY-82, } \\
\text { CMY-100, } \\
\text { CMY-157 }\end{array}$ & - & 6 \\
\hline $40-60$ & - & - & $\begin{array}{c}\text { 2: } \\
\text { CMY- } \\
130 \\
\text { CMY- } \\
132\end{array}$ & $\begin{array}{c}\text { 1: } \\
\text { CMY- } \\
21\end{array}$ & - & $\begin{array}{c}4: \\
\text { CMY-50, } \\
\text { CMY-65, } \\
\text { CMY-90, } \\
\text { CMY-114 }\end{array}$ & 2 & 5 \\
\hline $60-80$ & - & - & $\begin{array}{c}\text { 3: } \\
\text { CMY-44, } \\
\text { CMY- } \\
\text { 121, } \\
\text { CMY- } \\
132\end{array}$ & $\begin{array}{c}4: \\
2 \mathrm{x} \\
\mathrm{CMY}- \\
44, \\
2 \mathrm{x} \\
\mathrm{CMY}- \\
161\end{array}$ & - & $\begin{array}{c}5: \\
\text { CMY-68, } \\
\text { CMY-72, } \\
\text { CMY-89, } \\
\text { CMY-90, } \\
\text { CMY-114 }\end{array}$ & 3 & 9 \\
\hline$\geq \mathbf{8 0}$ & 3 & 2 & $\begin{array}{c}\text { 2: } \\
\text { CMY- } \\
130 \\
\text { CMY- } \\
132\end{array}$ & $\begin{array}{c}\text { 2: } \\
\text { CMY- } \\
153 \text {, } \\
\text { CMY- } \\
161\end{array}$ & - & $\begin{array}{c}\text { 1: } \\
\text { CMY-68 }\end{array}$ & 5 & 3 \\
\hline
\end{tabular}

\section{Total: $10 \quad 43$}

Abbreviations: KMA, $k$-mer alignment method; SRST2, short read sequence typer version 2.

${ }^{a}$ Alleles detected by bioinformatic tools KMA version 1.34 and SRST2. Number in bold indicates total number of alleles detected in the 10 replicates. Enzyme-allele are listed below for each CMY-category and CMY-template coverage range.

${ }^{\mathrm{b}}$ Totals are listed for each CMY-template coverage category (row totals), as well as the total number of gene-alleles predicted for each tool (Total:) 


\section{Figure 6.1. Accurate ARG detection is dependent on isolate coverage in}

metagenome. (Page 182). Mock metagenomes containing a beef fecal metagenome mixed with $S$. ser. Heidelberg reads at 0.1-, 1-, 2-, 5-, and 10-X genome coverage $(\mathrm{n}=10$ at each coverage level) were evaluated for presence of ARGs using both KMA $(\square)$ and SRST2 $(\times)$ in silico tools. Beef metagenome without added S. ser. Heidelberg reads was analysed as a control $(0 \mathrm{X}$ panel, $\mathrm{n}=1)$. Percent ARG detection (y-axis) of 10 replicates, with upper and lower 95\% confidence intervals (dashed lines), are plotted as a function of detected ARG template gene coverage (x-axis). CMY-2 panel (right y-axis label, top row), refers to the gene-allele (bla $\left.a_{\mathrm{CMY}-2}\right)$ detected in the original isolate assembly; CMY-2 clade (middle row), refers to detection of alleles within the same phylogenetic clade as the target gene (i.e. related to $b l a_{\mathrm{CMY}-2}$ ); Other CMY Alleles (bottom row), refers to alleles of the target gene family that are not as closely related to the target gene ( $\leq 90 \%$ nucleotide identity to CMY -2$)$. Darker point-color intensity is a result of multiple points (different gene-alleles) overlapping. Where multiple points are present (e.g. Bottom right: $10 \mathrm{X}-$ Other CMY Alleles - $\geq 80$ $\%$ coverage there are $4 \times \mathrm{s}$ ), each point represents a different allele (e.g. bla $a_{\mathrm{CMY}-114}$, $b l a_{\mathrm{CMY}-81}, b l a_{\mathrm{CMY}-90}$, and $b l a_{\mathrm{CMY}-83}$ were all detected by SRST2 and are each denoted by an $X)$. 


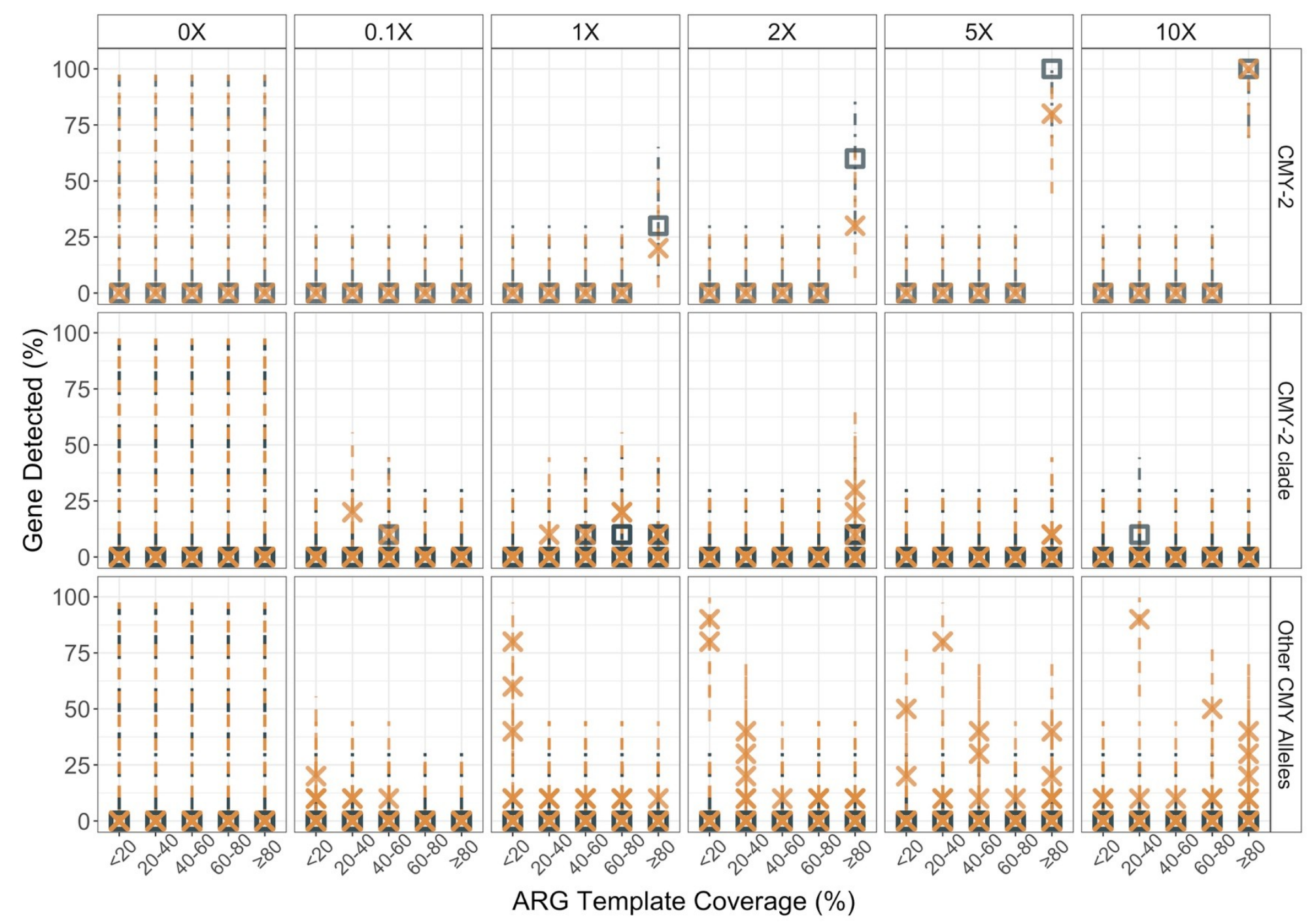

Tool $\square$ KMA X SRST2 


\subsubsection{Proportion of Isolate Reads in Metagenome Required for ARG Detection}

Small scale estimation of the ratio of isolate : metagenome reads for 3,4 , and 5 Mbp isolate genome sizes at 5- and 10-X genome coverage demonstrated that as the number of reads in a metagenomic sequence increase, the proportion of reads that the isolate sequence must constitute for ARG detection decreases (Figure 6.2). However, this is isolate genome-size and coverage dependent, where smaller sized organisms require fewer reads for accurate ARG detection.

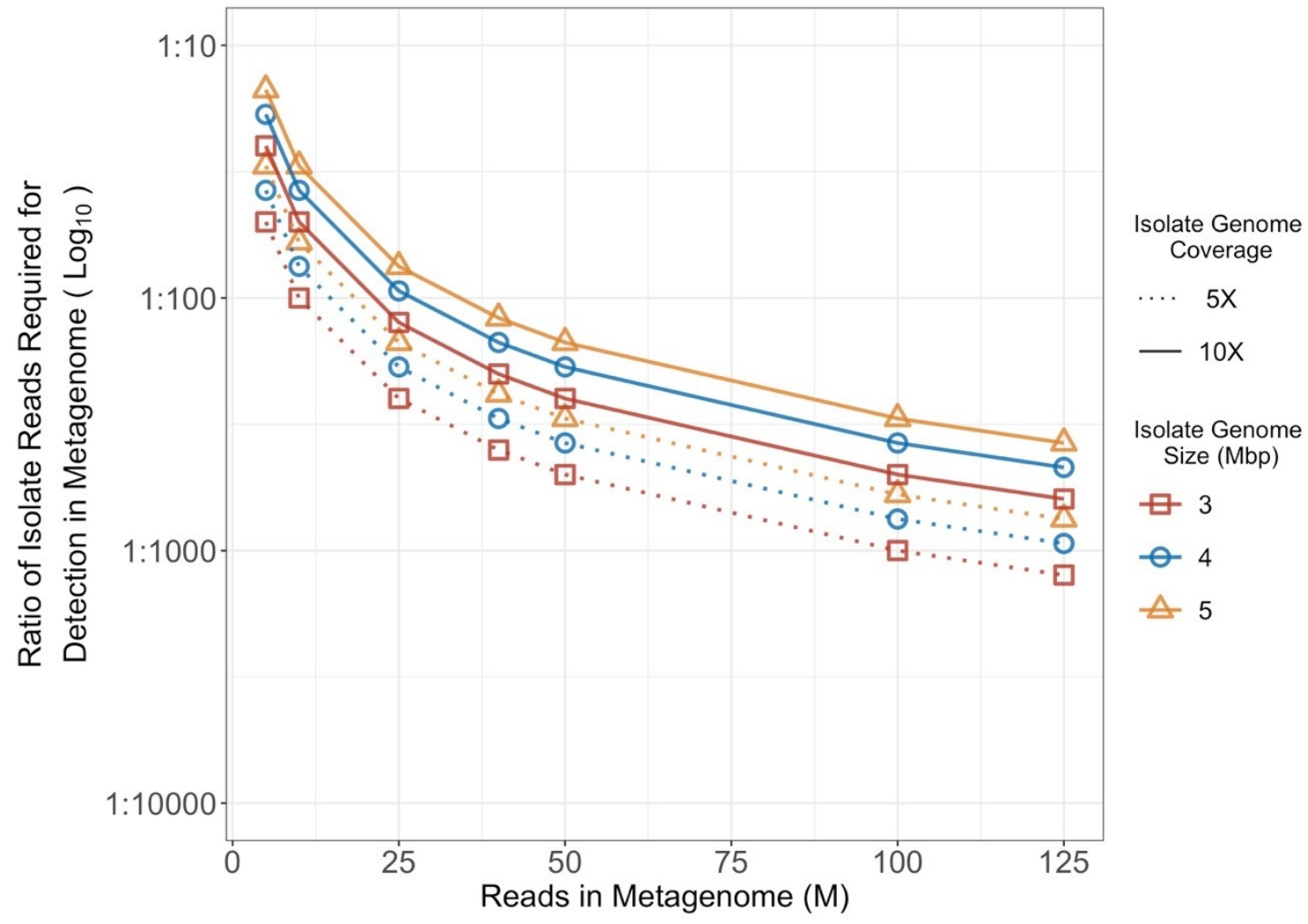

Figure 6.2. The fewer the number of bacterial reads in a metagenome, the higher the proportion the target bacteria must constitute in order to accurately detect ARGs. The ratio of isolate reads required for ARG detection in metagenome ( $\log _{10} \mathrm{y}$-axis) was plotted as a function of total reads in metagenome ( $\mathrm{x}$-axis, $\mathrm{M}=$ million). Estimates were conducted for a "best case scenario", where all reads in metagenome mapped to bacteria. Isolate genome sizes of 3,4 , and $5 \mathrm{Mbp}$ (million base pairs) are differentiated by point shape and colour. For each genome size (colour), isolate genome coverage levels are differentiated by linetype: $5 \mathrm{X}$ coverage, dotted; $10 \mathrm{X}$ coverage, solid. 


\subsection{Discussion}

This study corroborates that bacterial isolates must be present in a metagenome at an abundance sufficient to provide approximately $10 \mathrm{X}$ genome coverage in order for ARGs to be accurately detected at $\geq 80 \%$ ARG-target coverage. Whereas all tools accurately predicted phenotypic resistance using isolate WGSs (Cooper et al., 2020), with metagenomics there is a risk of reporting false-positives for closely related ARG-alleles if the bioinformatic method used permits reads to map to multiple genes in the database as does SRST2 (Figure 6.1, Table 6.3). This may also result in over-estimation of the ARG burden in a sample where multiple genes are reported at $\geq 80 \%$ identity but only one was actually present in the sample. At lower target-organism coverage ARGs may be detected at lower ARG target-coverage cutoffs (e.g. 40 - $60 \%$ ) (Figure 6.1). However, although the ARGs encoded by these low abundance organisms can be detected at lower cutoffs one must also be aware of possible detection of false-positives for alternative ARGalleles (Figure 6.1).

Technological advancements have greatly improved DNA collection and sequencing from complex samples. Ni et al. (2013) proposed a method to estimate the amount of metagenomic sequencing required when the abundances of different prokaryotes in a sample are known. However, in many complex sample matrices prokaryotic abundances are not known. Even if the prokaryotic composition was known, different DNA storage, extraction, and sequencing techniques would still introduce biases in the sequence community composition (reviewed by Nayfach and Pollard, 2016; Hugerth and Andersson, 2017; Brandt and Albertsen, 2018). As metagenomic sequencing only captures a fraction of the community within a DNA sample, it is unlikely all 
organisms will be equally present at high coverage levels. In fact, studies have found microbial communities within complex samples to be highly uneven, with $3-4$ orders of magnitude difference in abundance between organisms (Sogin et al., 2006; Hugerth and Andersson, 2017).

Considering a metagenome of 40 million reads where all reads are bacterial (a “clean" sample), a $5 \mathrm{Mbp}$ organism would need to constitute approximately $0.8 \%$ of the metagenome to be present at 10X coverage (Table 6.2, Figure 6.2). However, in complex matrices such as those found in agri-food production, host DNA may comprise 10 to $90 \%$ of the metagenome (Noyes et al., 2016; Lloyd-Price et al., 2017; Pereira-Marques et al., 2019), and microbiome profiling becomes more inaccurate as the level of host DNA in a sample increases (Pereira-Marques et al., 2019). Therefore, if only $10 \%$ of 40 million reads map to bacteria a $5 \mathrm{Mbp}$ bacterial genome would have to amount to approximately $8.3 \%$ of the bacterial reads in the sample for $10 \mathrm{X}$ coverage enabling accurate ARG detection. One must consider the likelihood that a target organism would comprise $8 \%$ of the bacteria in a complex sample. Minor bacterial populations would likely be missed.

A goal of sequencing preparation is removal of host DNA and enrichment of low abundance material (Payne et al., 2020). Methods for removal of host DNA during extraction (Hopkins et al., n.d.; Gabor et al., 2003; Burke et al., 2009; Hasan et al., 2016; Stiefel et al., 2016; Nelson et al., 2019) as well as bioinformatic methods to remove host reads prior to sequencing have been developed (Haque et al., 2015; Castro et al., 2018; Clarke et al., 2019; Czajkowski et al., 2019; Bush et al., 2020). Nonetheless, methods for removal of eukaryotic DNA post-sequencing bioinformatically become more difficult 
when a sample may contain large complex mixtures of eukaryotes including plants and animals along with microbial eukaryotes.

An alternative method using targeted bait-capture techniques have been employed recently in a number of studies (Lanza et al., 2017; Lee et al., 2017; Noyes et al., 2017; Gaudin and Desnues, 2018; Guitor et al., 2019). In this target-baiting technique biotinylated "baits" complementary to desired target sequences (e.g. ARG sequences) are utilized to selectively bind and extract target DNA fragments from total DNA extracts. Work by Lanza et al. (2017) utilized a targeted sequence capture system to analyse the resistome of human and swine fecal samples which enriched target sequence detection of ARGs 279-fold to shotgun sequencing alone. Targeted enrichment or targeted genome capture (TGC) of pathogens has also been utilized to enrich specific DNA sequences (Lee et al., 2017). Lee et al. (2017) found a number of veterinary pathogens detected using PCR were not isolated by targeted genome capture (TGC) next generation sequencing (NGS).

Alternatively, a recently developed tool "Read Until" by Payne et al. (2020) for Nanopore long read sequencing utilizes adaptive sequencing where targets can be selected for and adjusted during a run, in real time, allowing the user to select and modify which genes are enriched during sequence read generation. This technique allows not only for enrichment of targets, but also rejection or depletion of undesired host sequence data as reads are generated (Payne et al., 2020). As parameters can be set to stop sequencing targets once they reach a pre-determined desired coverage level, this method would "balance" the spread of coverage among the population, possibly allowing ARG detection in low abundance organisms. 
Although we were able to successfully detect the CMY-2 enzyme in mock metagenomes at $10 \mathrm{X}$ coverage, this was using a metagenome that did not contain organisms with closely related resistance genes. For example, many Enterobacteriaceae species encode chromosomal $\beta$-lactamase resistance genes such as $b l a_{\mathrm{ACT}}$ and $b l a_{\mathrm{CMY}}$ alleles in some Enterobacter and Citrobacter species, respectively. Presence of closely related ARGs within a metagenome may affect read-mapping and should be investigated further.

Metagenomics is an extremely valuable technology. However, current methodologies may not yet be effective for monitoring AMR in complex matrices such as agri-food samples using metagenomics. Nonetheless, it is vital that the limitations be understood if data is to be used to inform risk assessment. 


\section{Chapter 7: Concluding Remarks}

AMR is one of the most significant threats to public health. There have been many investigations into the consequences of antimicrobial use in agriculture and livestock production. Antimicrobial use in food-producing animals not only facilitates spread of resistance to bacteria in food products but may also result in dissemination of antimicrobial residues into the environment (Manyi-Loh et al. 2018; Merlin, 2020) . While in some cases abolishing or limiting use of an antimicrobial has successfully slowed the spread of AMR (van den Bogaard et al., 2000; Aarestrup et al., 2001; Phillips et al., 2004; Cogliani et al., 2011; Levy, 2014; Tang et al., 2017), in other cases resistance is still observed in the absence of use (although this may be due to co-selection with another antimicrobial) (Hasman and Aarestrup, 2002; Phillips et al., 2004; Lai et al., 2011; Merlin, 2020). In fact, the frequency of resistance to third-generation cephalosporins in Salmonella from broiler chickens decreased following a ban on the use of very important antimicrobials by the Canadian poultry industry (see Chapter 1, Table 1.2) (Government of Canada, 2020). Although this is promising, AMR patterns vary between countries depending on the degree of antibiotic consumption (Llor and Bjerrum, 2014; Manyi-Loh et al., 2018). As geographical boundaries do not stop the spread of bacteria nor AMR, it is imperative that surveillance and monitoring of resistance continue even in countries that have restricted or begun limiting/regulating antimicrobial use.

\subsection{Thesis Objectives}

The overarching goal of my doctorate research was to fill in current knowledge gaps for transmission and detection of AMR in food production, and to evaluate 
methodology for resistance surveillance in agri-food samples. Although current regulations require testing of agri-food products (including foods, feeds, and fertilizers) for pathogenic bacteria, the methods used by routine testing laboratories are intended and optimized for isolation of specific organisms. As such, these methods may not be sufficient for routine detection of resistance in foodborne bacteria, and therefore not effective for monitoring the resistance burden in food production.

In this thesis I studied the utility of genomics-based techniques for AMR gene (ARG) detection of resistance in agri-food samples and investigated the transfer of AMR between foodborne bacterial isolates. There were three main objectives of this thesis: (i) evaluate the accuracy of genotype in predicting resistance phenotypes; (ii) investigate the benefits and mechanisms of resistance in food production environment(s); and (iii) evaluate whether metagenomics is suitable for high-throughput resistance surveillance in agri-food products.

\subsection{Summary of Research Chapters}

Although recent studies have established a concordance of AMR phenotype prediction using WGS-based on detection of genetic markers (Tyson et al., 2015, 2016; McDermott et al., 2016; Zhao et al., 2016), few of these studies compared available in silico ARG-detection tools and databases. In addition, information regarding WGS requirements for accurate ARG determination in isolates had not been addressed. Chapter 2 confirmed, consistent with other studies, that AMR phenotype could be predicted using WGS data with all bioinformatic tools tested. However, SNVs resulting in mutations conferring aminoglycoside resistance were difficult to determine and not resolved in all 
cases. Of note was the induction of high levels of streptomycin (STR) resistance in all Salmonella isolates tested, even when STR-resistance was not encoded. In addition, it was observed that WGS-assembly was not required for ARG-detection provided sufficient sequence coverage was obtained. It was hypothesized that ARGs may be better detected using raw-read sequence data, as raw-reads aren't subject to errors introduced during assembly. Although this was the case for some of the ARGs tested, it was both coverage- and gene- dependent, and significance differed depending on the assembly method. The high accuracy of ARG-detection using raw-read data is important if metagenomics is to be used for AMR surveillance in food production.

Similarly, Chapter 3 confirmed that phenotype can be accurately predicted from bacterial WGS data for well-studied bacterial species. Chapter 3 investigated use of culture and molecular based methods to detect carbapenem-resistance in unknown bacteria from wastewater samples. In contrast to Chapter 2 a number of the strains isolated did not encode any ARGs known to confer carbapenem-resistance or -tolerance. However, many of these discrepancies were resolved by lowering ARG-detection targetcutoffs from $\geq 90$ to $\geq 80 \%$ identity, and the remaining isolates with unidentified carbapenem-tolerance were mostly Pseudomonas species which likely have intrinsic resistance mechanisms. This chapter also emphasizes the utility of isolate WGS for detection of ARGs, especially over molecular techniques such as PCR, when AMR may result from any of a large number of resistance genes. Whereas using five to six multiplex PCRs to search for 13 carbapenem- or $\beta$-lactam -resistance genes was not always able to determine the mechanism of resistance, WGS allowed additional investigation(s) of many alternative ARGs. 
Whereas Chapters 2 and 3 studied the concordance between genotype and phenotype in determining the AMR profile of an isolate, Chapters 4 and 5 attempted to determine if and how resistance in food production should be monitored. Persistent contamination of bacterial pathogens in food manufacturing environments is a major concern, as these pathogens may transfer to food products resulting in consumer illness (Lundén et al., 2002; Gaulin et al., 2012; Ferreira et al., 2014; Leong et al., 2014; Martínez-Suárez et al., 2016; Ortiz et al., 2016). Although biofilm formation is a wellknown mechanism of both resistance and persistent bacterial contamination (Møretrø and Langsrud, 2004; Carpentier and Cerf, 2011; Wang et al., 2014; Bridier et al., 2015), resistance to sanitizers is also hypothesized to enable persistent contamination in manufacturing environments (Wang et al., 2014; Martínez-Suárez et al., 2016; Ortiz et al., 2016).

In contrast to previous studies which investigated L. monocytogenes persistence in food production using a small number of isolates, Chapter 4 analysed WGS data of 1279 L. monocytogenes strains sequenced as part of routine food product testing over 10 years at the CFIA. Five of the most frequently isolated clonal complexes (CCs) of $L$. monocytogenes were significantly more likely to encode gene(s) conferring QAC resistance. Consistent with other studies all but one of the CC321 isolates, one of the most commonly isolated CCs, encoded the QAC-resistance cassette $b c r A B C$ (Harrand et al., 2020). Notably, in almost all resistant isolates, QAC-resistance was plasmid-borne and appeared to belong predominantly to one of three plasmid types. This suggests that transfer of plasmid-borne sanitizer resistance may be associated with pathogen 
persistence in food production. However, additional studies including risk assessment need to be conducted to confirm this relationship.

To further investigate the impact of resistant MGEs in food production, Chapter 5 tested the ability of common foodborne commensal bacteria to act as recipients to a foodborne pathogen (Salmonella enterica ser. Heidelberg). A plasmid carrying a bla $a_{\mathrm{CMY} \text { - }}$ 2-blc-sugE cassette, conferring resistance to third generation cephalosporins ( $3 \mathrm{GCs}$ ) (bla $\left.a_{\mathrm{CMY}-2}\right)$ and QACs (sugE), was successfully transferred to Citrobacter, Enterobacter, Hafnia, and Klebsiella strains isolated from food products. Conjugation occurred under different nutrient and temperature conditions for most recipient strains, in many cases at relatively high frequencies. Although there are many publications investigating conjugation between bacterial strains, this is the first investigation of transfer between a foodborne pathogen and multiple commensal foodborne bacterial strains. Plasmid transfer frequency was high for some recipients $\left(10^{-3}\right.$ to $10^{-2} \mathrm{CFU} / \mathrm{mL}$ transconjugants / $\mathrm{CFU} / \mathrm{mL}$ recipients per hour), even at $25^{\circ} \mathrm{C}$ on minimal media. These results provide evidence that high rates of plasmid transfer may occur in food production environments, even at room temperature, and that commensal food bacteria that are not routinely tested could facilitate transfer of AMR to foodborne pathogens, connecting AMR in agri-food and clinical settings.

The final chapter leveraged the results of Chapters 2 and 3 to determine whether metagenomics, a high-throughput NGS technology, could be utilized to monitor resistance in complex agri-food samples. Chapter 6 tested the hypothesis that some ARGs in agri-food samples may be below the limit of detection (LOD) for metagenomic detection. Metagenomics is quickly gaining popularity as a method to study and compare 
AMR and microbial communities in different sample matrices (Jernberg et al., 2010; Cantas et al., 2013; Fitzpatrick and Walsh, 2016; Noyes et al., 2016; Thomas et al., 2017), as it allows simultaneous analysis of ARGs and community composition without requiring culturing. However, bias is introduced at all stages of sample processing and analyses, and this bias coupled with high amounts of host DNA may result in lowabundance organisms that would have been detected by culturing techniques being missed.

This chapter was a pilot study investigating the LOD for ARGs in agri-food samples using metagenomics. Similar to Chapters 2 and 3, when the target AMR bacteria was present at $10 \mathrm{X}$ genome coverage in a metagenome it was accurately detected by all tools. Results also found the target ARG was almost always detected at $5 \mathrm{X}$ genome coverage, however this was using a $\geq 80 \%$ target-gene cutoff (suggested by results of Chapter 3). Additionally, estimation of the proportion of reads required to accurately detect $\mathrm{ARGs}$ at $5 \mathrm{X}$ and $10 \mathrm{X}$ coverage confirm that as more sequencing reads are produced the target can comprise a smaller proportion of the bacterial population. However, agri-food samples are often complex and may contain large amounts of eukaryotic DNA thereby requiring target bacteria in the sample be present at high levels, or requiring deeper sequencing be achieved.

\section{Chapter 8: Future Directions}

The work published in this thesis has provided valuable information as to the requirements for AMR detection in bacterial isolates from complex matrices, such as those found in agri-food production. This research has determined sequence coverage 
requirements for accurate $\mathrm{ARG}$ detection, provided evidence for resistance resulting in persistence of foodborne pathogens in agri-food production environments, and confirmed that commensal agri-food bacteria can facilitate transfer of ARGs throughout environments in the food production continuum.

Not surprisingly, variations in resistance phenotype were observed under different nutrient conditions in both Chapters 2 and 5. Differences in nutrient conditions would be expected to have some effect on resistance, as different nutrients may up- or downregulate different genetic elements and/or induce bacterial stress responses resulting in phenotype variations (Testerman et al., 2006; Poole, 2012; Li et al., 2016; Liu et al., 2019). Still, this is an important concept to consider when deciding which approach to use for investigating the resistome of a complex sample, as methods involving plating and phenotyping may only isolate well-known culturable organisms. Additionally, this emphasizes the variability of phenotypic resistance where even "known" culturable organisms may be missed due to down-regulation of resistance preventing growth. In contrast, the results of Chapter 3 demonstrated that blindly plating and selecting colonies from a complex, AMR-burdened, bacterial population (such as wastewater) using a semiselective media may result in isolation of non-target organisms that do not harbour critically important ARGs. Further, Chapter 5 highlights another consideration when using culture-based approaches in that naïve bacterial hosts with newly acquired mobile genetic elements (MGEs) may have not yet co-evolved or -adapted with their MGE(s). As reported in Chapter 5, most Enterobacteriaceae recipients received the $b l a_{\mathrm{CMY}-2}$ plasmid conferring resistance to cefotaxime (CTX). However, some recipients exhibited significantly higher transfer frequencies when plating on the lower $1 \mu \mathrm{g}$ CTX 
concentration, or only grew on $1 \mu \mathrm{g}$ CTX, suggesting that certain culture-based conditions may result in underestimation of AMR burden in some cases. Collectively, these results suggest culture-based phenotypic methods are useful for determining the bacterial load of a sample; measuring tolerance or resistance levels of isolates; or for enrichment and selection of desired species, assuming there is a selective method available; but may underestimate the total AMR burden of a sample's bacterial population.

Although it may not be possible to leverage isolation methods used in routine food testing to study AMR, surveillance of biocide resistance genes may still be feasible as suggested by the high incidence in Chapter 4. Results from both Chapter 4, persistence of plasmid-borne QAC resistance, and Chapter 5, high conjugation rates of a $3 \mathrm{GC}$ plasmid between foodborne bacteria, suggest plasmid-transfer of resistance determinants in the food production continuum may play an important role in resistance gene dissemination. Studies have shown critically important AMR may be co-selected (Brown et al., 2019), and subinhibitory concentrations of antibiotics have been found to greatly increase transfer frequency (Scornec et al., 2017; Merlin, 2020). Future work should investigate the effects of subinhibitory concentrations of sanitizers and antimicrobial residues on plasmid transfer frequency, as well as a larger number of commensal agrifood strains to determine whether donor-recipient relatedness influences transfer rates.

The findings of this thesis suggest WGS may be superior to PCR and culturebased typing methods for evaluating complex microbial communities. In contrast, Schwartz et al. (2003) used PCR to investigate the presence of vanA, mecA, and $\operatorname{ampC}$ in water biofilms and wastewater, and found the vanA gene in both wastewater and drinking 
water biofilms, even in the absence of enterococci (Anjum et al., 2017). Although, these genes could have been attributed to bacteria other than enterococci.

The use of metagenomics has been discussed as a possible high-throughput alternative to culturing and PCR for monitoring resistance genes in agri-food samples. Nonetheless, Suttner et al. (2020) were unable to detect any pathogenic E. coli in creeksediment metagenomes even though samples had tested positive using culture-based methods. This stresses that understanding the LOD of metagenomics is crucial if it is to be used to compare various sample types. Zaheer et al. (Zaheer et al., 2018) found a depth of D0.5 (one doubling dilution) was suitable to describe the microbiome and resistome of bovine fecal samples. However, their study extracted and sequenced DNA from actual bovine fecal samples in contrast to the synthetic metagenomes created for this thesis. As they did not spike any known ARGs into their samples prior to processing it is difficult to confirm what proportion of the bacterial population a doubling dilution of the sample represented, and whether this dilution would miss low-abundance organisms and ARGs within the sample. An additional limitation of metagenomics is that if an ARG is detected in a metagenome it is difficult to associate that ARG with a particular bacterial isolate (as obtaining high quality assemblies of all organisms in metagenomes is extremely difficult).

The findings that either bacterial isolates must: 1) constitute a large proportion of a sample's bacterial population or 2) a higher number of sequencing reads must be generated in order to detect low-abundance ARGs; suggests short-read metagenomics may not yet be suitable for studying complex agri-food sample matrices. An alternative possibility would be bait-capture, where selected genes are "baited" or enriched from 
DNA extracts prior to sequencing. Lanza et al. (2017) utilized a targeted sequence capture system to analyse the resistome of human and swine fecal samples. In this targetbaiting technique biotinylated "baits", complementary to desired target sequences (e.g. ARG sequences), are utilized to selectively bind and extract desired DNA fragments from total DNA extracts. This baiting technique enriched target sequence detection 279-fold compared to shotgun sequencing alone. Alternatively, recent progress in long-read sequencing technologies may allow generation of long reads from metagenomes that can span repetitive sequences thereby linking ARGs with flanking regions and possibly even host bacteria (Bertrand et al., 2019; Boolchandani et al., 2019; Che et al., 2019). As discussed previously, Payne et al. (2020) have developed the tool "Read Until" that utilizes adaptive sequencing to allow users to enrich or deplete selected targets during sequencing. This technique could possibly overcome limitations of short- and long-read metagenomic sequencing as low-abundance reads, below the LOD of short-read metagenomics, could be selectively enriched.

Another important alternative consideration stems from the data of Che et al. (2019) which suggested that most of the ARGs detected in WWTPs were carried on plasmids. Studies of AMR in agri-food samples may want to move away from both isolation/testing of specific organisms, and metagenomics, and focus on MGEs being transferred throughout food production (e.g. plasmidome sequencing). As mentioned previously, work has been conducted to investigate the plasmidome of complex sample matrices. Perez et al. (2020) reported that genetic information from sequencing of the plasmidome was novel in comparison to shotgun metagenomics of the same environment (only $30 \%$ of the plasmidome reads aligned with metagenomic reads). As plasmids 
provide a mechanism to distribute genes beneficial for survival the plasmidome is likely to contain an abundance of genes encoding virulence factors; antimicrobial-, biocide-, and metal-resistance; and functions involved in host-bacterial interactions (Dib et al., 2015). Therefore, the plasmidome may (arguably) contain resistance genes of highest risk and clinical relevance in agri-food production samples.

The findings of this thesis stress the importance of surveillance and monitoring of antimicrobial resistance in agri-food production, as resistance may readily transmit between commensal food bacteria and pathogens. Future research should compare various methods for resistance gene detection in agri-food samples, including enrichment and plating, real-time or quantitative PCR screening for genes of interest, plasmidome and long-read metagenomics, and bait-capture genomic techniques. 


\section{Appendices}

\section{Appendix A - Chapter 1}

\section{A.1 Methods for Figure 1.3 data analysis}

Metadata AMR files for whole-genome sequence data of Acinetobacter, Citrobacter, Enterobacter, Klebsiella, Salmonella, Escherichia coli and Shigella, were downloaded from the NCBI Pathogen Detection database on August 26, 2020 (https://www.ncbi.nlm.nih.gov/pathogens/). Isolation source categories were manually assigned based on provided sequence submission information (e.g. chicken breast was labelled as meat, cheese was labelled as dairy, etc). Data was excluded from analysis where source information was missing or incomplete. Clinical data was defined as data entries with the epi_type input as "clinical" and source as "Homo Sapiens". 


\section{Appendix B - Chapter 2}

\section{B.1 Tables S2.1, Table S2.2, Table S2.3}

The data file containing Table S2.1, Table S2.2, and Table S2.3 (labelled S1, S2, and S3 in publication) can be found online at:

https://www.frontiersin.org/articles/10.3389/fmicb.2020.00549/full\#suppleme ntary-material.

Table S2.1 - Sequence and isolate identifiers, serotyping data

Table S2.2 - Phenotyping data

Table S2.3 - Plasmid predictions and associated AMR (MOB-suite version 1.4.1) 


\section{Appendix C - Chapter 3}

\section{C.1 Supplementary data tables S3.1 and S3.2}

Supplementary data tables for sequence, AMR and PCR data (Table S3.1) and plasmid typing with associated AMR genes (Table S3.2, MOB-suite version 3.0.0) can be found online following peer review and acceptance at:

Cooper, Ashley (2020): Table S3.1. figshare. Dataset.

https://doi.org/10.6084/m9.figshare.13309529.v1

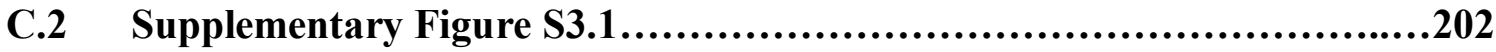




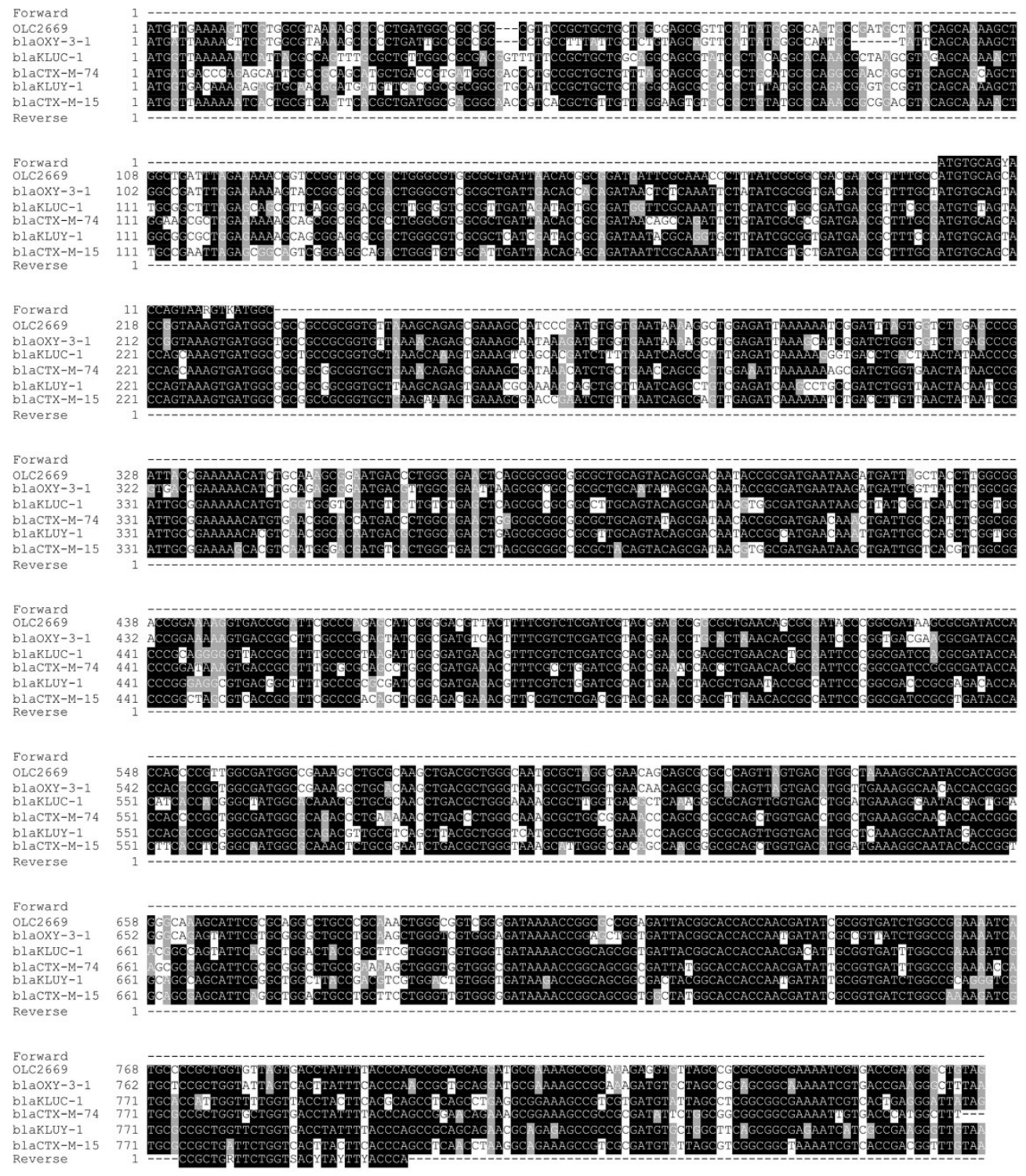

Figure S3. 1. Nucleic acid alignment of select bla $_{\mathrm{CTX}-\mathrm{M}}$ and blaxY $_{\mathrm{OX}}$ variants with

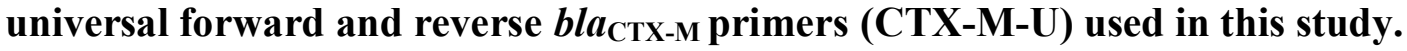
Black fill indicates identical nucleotides. Forward and Reverse primers contain the degenerate bases: $\mathrm{Y}$, is a pyrimidine; $\mathrm{R}$, is a purine; $\mathrm{K}$, is $\mathrm{G}$ or $\mathrm{T}$; $\mathrm{S}$, is $\mathrm{G}$ or $\mathrm{C}$. Alignment was created using T-Coffee (Di Tommaso et al., 2011). Alignment image generated using ExPASy BOXSHADE. Gene accessions: OLC2669, this study; blaOXY-3-1, AF491278; blaKLUC-1, AY026417; blaCTX-M-74, GQ149243; blaKLUY-1, AY623932; blaCTXM-15, FJ815288. 


\section{Appendix D - Chapter 4}

\section{D.1 Table S4.1}

Table S4.1 containing isolate identifiers, sources, and sequence information (including QAC resistance genes) can be found online at:

https://meridian.allenpress.com/jfp/article/doi/10.4315/JFP-20-328/445882/Quaternaryammonium-compound-resistance-as-a

D.2 Table 4.2 ..............................................................204 
Table 4.2. Genetic profiles of $L$. monocytogenes used in phenotypic resistance studies ${ }^{\mathrm{a}}$

\begin{tabular}{|c|c|c|c|c|c|c|c|c|c|c|}
\hline Isolate ID & Source & $\mathrm{CC}$ & MLST & $b c r A$ & $b c r B$ & $b c r C$ & emrE & $q a c C$ & PCR & MIC \\
\hline CFIAFB20140002 & Frozen Herring Fillet & 31 & 31 & - & - & - & - & - & - & 10 \\
\hline CFIAFB20090032 & RTE meat: beef strips & 11 & 11 & - & - & - & - & - & - & 4 \\
\hline CFIAFB20090080 & Leafy greens & 375 & 375 & - & - & - & - & - & - & 4 \\
\hline CFIAFB20090092 & RTE meat: wieners & 8 & 120 & - & - & - & 100.0 & - & - & 10 \\
\hline CFIAFB20090136 & Marinated frozen herring & 5 & 5 & - & - & - & - & - & - & 4 \\
\hline CFIAFB20090178 & Fish roe & 37 & 37 & - & - & - & - & - & - & 4 \\
\hline CFIAFB20100015 & Comtomme raw cheese & 415 & 361 & - & - & - & - & - & - & 4 \\
\hline CFIAFB20100043 & RTE meat: unknown & 204 & 204 & - & - & - & - & - & - & 4 \\
\hline CFIAFB20100115 & Gorgonzola cheese & 101 & 38 & - & - & - & - & - & - & 6 \\
\hline CFIAFB20100161 & RTE fish: smoked salmon & 6 & 6 & - & - & - & - & - & - & 4 \\
\hline CFIAFB20110012 & RTE meat: foie Gras & 121 & 121 & - & - & - & - & 99.19 & - & 10 \\
\hline CFIAFB20110038 & Liquid egg yolk, $1 \%$ salted & 14 & 360 & - & - & - & - & - & - & 4 \\
\hline CFIAFB20110039 & Fresh shredded lettuce & 37 & 37 & - & - & - & - & - & - & 4 \\
\hline CFIAFB20120118 & Dairy & 1 & 1 & - & - & - & - & - & - & 4 \\
\hline CFIAFB20120121 & Blue cheese & 224 & 224 & - & - & - & - & - & - & 4 \\
\hline CFIAFB20120126 & Diced red onion & 155 & 155 & - & - & - & - & - & - & 6 \\
\hline CFIAFB20120136 & Shelled peas & 331 & 1394 & - & - & - & - & - & - & 4 \\
\hline
\end{tabular}




\begin{tabular}{|c|c|c|c|c|c|c|c|c|c|c|}
\hline CFIAFB20120154 & Fresh lobster mushroom & ST1571 & ND & - & - & - & - & - & - & 4 \\
\hline CFIAFB20120163 & Fresh Pesto & 1 & 1 & - & - & - & - & - & - & 4 \\
\hline CFIAFB20130054 & Environmental: RTE meat & 204 & 204 & - & - & - & - & - & - & 4 \\
\hline CFIAFB20130065 & Sliced mushrooms & 7 & 7 & - & - & - & - & - & - & 4 \\
\hline CFIAFB20130115 & RTE meat: unknown & 9 & 9 & - & - & - & - & - & - & 4 \\
\hline CFIAFB20140037 & RTE meat: unknown & 9 & 9 & - & - & - & - & - & - & 4 \\
\hline CFIAFB20140056 & Cheese & 8 & 8 & - & - & - & - & - & - & 4 \\
\hline CFIAFB20140074 & RTE fish: smoked fish & 5 & 5 & - & - & - & - & - & - & 6 \\
\hline CFIAFB20140092 & RTE meat: beef balls & 5 & 5 & - & - & - & - & - & - & 4 \\
\hline CFIAFB20140104 & RTE meat: unknown & 5 & 5 & - & - & - & - & - & - & 4 \\
\hline CFIAFB20150003 & Environmental (unknown) & 5 & 5 & - & - & - & - & - & - & 4 \\
\hline CFIAFB20150036 & Environmental (unknown) & 1 & 1 & - & - & - & - & - & - & 4 \\
\hline CFIAFB20150043 & RTE fish & 321 & 321 & - & - & - & - & - & - & 4 \\
\hline CFIAFB20150088 & Fresh sliced mushrooms & 11 & 11 & - & - & - & - & - & - & 4 \\
\hline CFIAFB20170041 & Butter & 5 & 1093 & - & - & - & - & - & - & 4 \\
\hline CFIAFB20180210 & Fresh turnip Sticks & 388 & 388 & - & - & - & - & - & - & 4 \\
\hline CFIAFB20180008 & Flour & 7 & 7 & - & - & - & - & - & - & 4 \\
\hline CFIAFB20090004 & Smoked salmon & 5 & 5 & 100.0 & 100.0 & 100.0 & - & - & $\mathrm{A}, \mathrm{B}, \mathrm{C}$ & 14 \\
\hline
\end{tabular}




\begin{tabular}{|c|c|c|c|c|c|c|c|c|c|c|}
\hline CFIAFB20090012 & Genoa salami & 155 & 155 & 100.0 & 100.0 & 100.0 & - & - & $\mathrm{A}, \mathrm{B}, \mathrm{C}$ & 10 \\
\hline CFIAFB20090025 & RTE meat: BCon flakes & 9 & 9 & 100.0 & 100.0 & 100.0 & - & - & $\mathrm{A}, \mathrm{B}, \mathrm{C}$ & 14 \\
\hline CFIAFB20090118 & Frozen beef meatloaf & 321 & 321 & 100.0 & 100.0 & 99.71 & - & - & $\mathrm{A}, \mathrm{B}, \mathrm{C}$ & 12 \\
\hline CFIAFB20090130 & Environmental: meat & 11 & 11 & 100.0 & 100.0 & 100.0 & - & - & $\mathrm{A}, \mathrm{B}, \mathrm{C}$ & 14 \\
\hline CFIAFB20090156 & RTE fish: wild Sockeye Lox & 321 & 321 & 100.0 & 100.0 & 99.71 & - & - & $\mathrm{A}, \mathrm{B}, \mathrm{C}$ & 12 \\
\hline CFIAFB20100051 & Environmental: RTE meat & 204 & 204 & 100.0 & 100.0 & 100.0 & - & - & $\mathrm{A}, \mathrm{B}, \mathrm{C}$ & 14 \\
\hline CFIAFB20100068 & RTE meat: salami calabrese hot & 155 & 155 & 100.0 & 100.0 & 100.0 & - & - & $\mathbf{A}, \mathbf{B}, \mathbf{C}$ & 6 \\
\hline CFIAFB20100093 & RTE sandwich/pizza sub & 5 & 5 & 100.0 & 100.0 & 100.0 & - & - & $\mathrm{A}, \mathrm{B}, \mathrm{C}$ & 14 \\
\hline CFIAFB20100123 & RTE chicken pasta Caeser salad & 5 & 5 & 100.0 & 100.0 & 99.71 & - & - & $\mathrm{A}, \mathrm{B}, \mathrm{C}$ & 14 \\
\hline CFIAFB20100146 & Mushrooms & 7 & 7 & 100.0 & 100.0 & 100.0 & - & - & $\mathrm{A}, \mathrm{B}, \mathrm{C}$ & 12 \\
\hline CFIAFB20100157 & Environmental & 199 & 199 & 99.81 & 99.69 & 98.84 & - & - & $\mathrm{A}, \mathrm{B}, \mathrm{C}$ & 14 \\
\hline CFIAFB20100159 & RTE meat: sausage & 9 & 9 & 100.0 & 100.0 & 100.0 & - & - & $\mathrm{A}, \mathrm{B}, \mathrm{C}$ & 12 \\
\hline CFIAFB20110020 & Frozen canned lobster & 321 & 321 & 100.0 & 100.0 & 99.71 & - & - & $\mathrm{A}, \mathrm{B}, \mathrm{C}$ & 12 \\
\hline CFIAFB20110042 & RTE meat & 5 & 5 & 100.0 & 100.0 & 100.0 & - & - & $\mathrm{A}, \mathrm{B}, \mathrm{C}$ & 12 \\
\hline CFIAFB20110091 & Frozen fried sliced potatoes & 26 & 26 & 99.81 & 99.69 & 98.84 & - & - & $\mathrm{A}, \mathrm{B}, \mathrm{C}$ & 12 \\
\hline CFIAFB20110096 & Salad & 321 & 321 & 100.0 & 100.0 & 99.71 & - & - & $\mathrm{A}, \mathrm{B}, \mathrm{C}$ & 12 \\
\hline CFIAFB20110101 & Honeydew melon (fruit salad) & 321 & 321 & 100.0 & 100.0 & 99.71 & - & - & $\mathrm{A}, \mathrm{B}, \mathrm{C}$ & 12 \\
\hline CFIAFB20120102 & Environmental (unknown) & 321 & 321 & 100.0 & 100.0 & 99.71 & - & - & $\mathrm{A}, \mathrm{B}, \mathrm{C}$ & 12 \\
\hline
\end{tabular}




\begin{tabular}{|c|c|c|c|c|c|c|c|c|c|c|}
\hline CFIAFB20120106 & Environmental: meat & 321 & 321 & 100.0 & 100.0 & 99.71 & - & - & $\mathrm{A}, \mathrm{B}, \mathrm{C}$ & 12 \\
\hline CFIAFB20120156 & Cantaloupe chunks & 204 & 204 & 100.0 & 100.0 & 100.0 & - & - & $\mathrm{A}, \mathrm{B}, \mathrm{C}$ & 12 \\
\hline CFIAFB20120157 & Honeydew chunks & 204 & 204 & 100.0 & 100.0 & 100.0 & - & - & $\mathrm{A}, \mathrm{B}, \mathrm{C}$ & 12 \\
\hline CFIAFB20130027 & Italian style meats around fresh mozzarella & 321 & 321 & 100.0 & 100.0 & 99.71 & - & - & $\mathrm{A}, \mathrm{B}, \mathrm{C}$ & 12 \\
\hline CFIAFB20130044 & RTE fish: smoked salmon & 1 & 1 & 99.81 & 99.69 & 98.84 & - & - & $\mathrm{A}, \mathrm{B}, \mathrm{C}$ & 14 \\
\hline CFIAFB20130066 & Dairy & 31 & 325 & 100.0 & 100.0 & 100.0 & - & - & $\mathrm{A}, \mathrm{B}, \mathrm{C}$ & 10 \\
\hline CFIAFB20130090 & RTE meat-calabrese sopressa & 9 & 9 & 100.0 & 100.0 & 100.0 & - & - & $\mathrm{A}, \mathrm{B}, \mathrm{C}$ & 14 \\
\hline CFIAFB20140089 & RTE meat & 321 & 321 & 100.0 & 100.0 & 99.71 & - & - & $\mathrm{A}, \mathrm{B}, \mathrm{C}$ & 14 \\
\hline CFIAFB20150080 & RTE meat: beef Jerky & 3 & 3 & 100.0 & 99.69 & 99.71 & - & - & $\mathrm{A}, \mathrm{B}, \mathrm{C}$ & 14 \\
\hline CFIAFB20160003 & RTE fish: milkfish & 8 & 8 & 99.81 & 99.69 & 98.84 & - & - & $\mathrm{A}, \mathrm{B}, \mathrm{C}$ & 12 \\
\hline CFIAFB20160040 & Chocolate milk & 155 & 155 & 100.0 & 100.0 & 100.0 & - & - & $\mathrm{A}, \mathrm{B}, \mathrm{C}$ & 10 \\
\hline CFIAFB20160069 & RTE egg roll product & 199 & 199 & 99.81 & 99.69 & 98.84 & - & - & $\mathrm{A}, \mathrm{B}, \mathrm{C}$ & 12 \\
\hline CFIAFB20170131 & Fresh guacamole & 204 & 204 & 100.0 & 100.0 & 100.0 & - & - & $\mathrm{A}, \mathrm{B}, \mathrm{C}$ & 12 \\
\hline CFIAFB20170002 & Environmental & 5 & 5 & 100.0 & 100.0 & 100.0 & - & - & $\mathrm{A}, \mathrm{B}, \mathrm{C}$ & 12 \\
\hline CFIAFB20170050 & Butter & 5 & 1093 & 100.0 & 100.0 & 100.0 & - & - & $\mathrm{A}, \mathrm{B}, \mathrm{C}$ & 14 \\
\hline CFIAFB20170090 & Fresh sweet kale vegetable salad & 321 & 321 & 100.0 & 100.0 & 99.71 & - & - & $\mathrm{A}, \mathrm{B}, \mathrm{C}$ & 12 \\
\hline
\end{tabular}


Abbreviations: CC, clonal complex; RTE, ready-to-eat; MLST, multi-locus sequence type; MIC, minimum inhibitory concentration $(\mu \mathrm{g} / \mathrm{mL}$ benzalkonium chloride).

aSixty-nine L. monocytogenes isolates were analyzed by whole genome sequencing for sequence type (MLST), bcrABC, and alternative sanitizer resistance genes $q a c C$ and $e m r E$. Numbers in $b c r A, b c r B, b c r C, q a c H$, and $e m r E$ columns indicate percent identity of detected to gene to resistance gene in database. Isolates were also analyzed using a PCR technique targeting the $b c r A, b c r B$ and $b c r C$ genes (Minarovičová et al., 2018). Phenotypic resistance was not observed for isolate CFIAFB20100068 (bold). 


\section{Appendix E - Chapter 5}

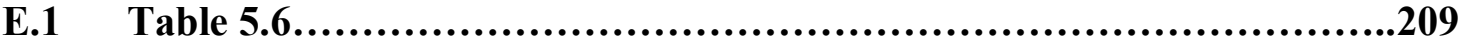

Table 5.6. Pairwise phylogenetic distance of strain relatedness compared to donor Salmonella enterica ser. Heidelberg donor.

\begin{tabular}{llc}
\hline Recipient Strain & Genus & $\begin{array}{l}\text { Pairwise Distance from } \\
\text { Donor }^{\mathbf{a}}\end{array}$ \\
\hline MG1655 & Escherichia & 0.05097811 \\
Cit2431 & Citrobacter & 0.05319638 \\
Cit1224 & Citrobacter & 0.05444577 \\
Cit1135 & Citrobacter & 0.05477116 \\
Cit3215 & Citrobacter & 0.05526501 \\
Cit2915 & Citrobacter & 0.05724877 \\
Ent2448 & Enterobacter & 0.08067947 \\
Ent2920 & Enterobacter & 0.08092081 \\
Ent3235 & Enterobacter & 0.08138428 \\
Ent2939 & Enterobacter & 0.08160683 \\
Ent1650 & Enterobacter & 0.081997 \\
Klb1227 & Klebsiella & 0.08316635 \\
Klb2894 & Klebsiella & 0.08344764 \\
K1b1651 & Klebsiella & 0.0834715 \\
Klb1672 & Klebsiella & 0.08375042 \\
Klb1237 & Klebsiella & 0.08376368 \\
Haf3292 & Hafnia & 0.22297334 \\
Haf1276 & Hafnia & 0.22311297 \\
Haf1306 & Hafnia & 0.22323112 \\
Haf1231 & Hafnia & 0.22326819 \\
Haf1525 & Hafnia & 0.22339124 \\
Prt2451 & Proteus & 0.3234643 \\
Prt3112 & Proteus & 0.32356841 \\
Prt1615 & Proteus & 0.32362591 \\
Prt1100 & Proteus & 0.32398764 \\
Prt3244 & Proteus & 0.32411245 \\
\hline Paiw1s dista &
\end{tabular}

${ }^{a}$ Pairwise distance from donor strain was calculated using the cophenetic.phylo function from the ape package in R statistical software v. 4.0.2 from the core genome phylogenetic tree output of bcgTree. 


\section{References}

Aarestrup, F.M., Seyfarth, A.M., Emborg, H.-D., Pedersen, K., Hendriksen, R.S. \& Bager, F. (2001). Effect of Abolishment of the Use of Antimicrobial Agents for Growth Promotion on Occurrence of Antimicrobial Resistance in Fecal Enterococci from Food Animals in Denmark. Antimicrobial Agents and Chemotherapy, 45, 2054-2059.

Aarestrup, F.M. \& Woolhouse, M.E.J. (2020). Using sewage for surveillance of antimicrobial resistance. Science, 367, 630-632.

Alderliesten, J.B., Duxbury, S.J.N., Zwart, M.P., Visser, J.A.G.M. de, Stegeman, A. \& Fischer, E.A.J. (2020). Effect of donor-recipient relatedness on the plasmid conjugation frequency: a meta-analysis. BMC Microbiology, 20, 135.

Al-Masaudi, S.B., Day, M.J. \& Russell, A.D. (1991). Effect of some antibiotics and biocides on plasmid transfer in Staphylococcus aureus. Journal of Applied Bacteriology, 71, 239-243.

Almeida, A.C.S., Cavalcanti, F.L.S., Martins, W.M.B., Vilela, M.A., Gales, A.C., Morais Junior, M.A. \& Morais, M.M.C. (2013). First Description of KPC-2-Producing Klebsiella oxytoca in Brazil. Antimicrobial Agents and Chemotherapy, 57, 40774078.

Anderson, K.L., Whitlock, J.E. \& Harwood, V.J. (2005). Persistence and Differential Survival of Fecal Indicator Bacteria in Subtropical Waters and Sediments. Applied and Environmental Microbiology, 71, 3041-3048.

Andersson, D.I. \& Hughes, D. (2014). Microbiological effects of sublethal levels of antibiotics. Nature Reviews Microbiology, 12, 465-478.

Andrews, S. (2010). FastQC A Quality Control tool for High Throughput Sequence Data [Internet document] URL https://www.bioinformatics.babraham.ac.uk/projects/fastqc/. Accessed 05/03/2018.

Anjum, M.F., Zankari, E. \& Hasman, H. (2017). Molecular Methods for Detection of Antimicrobial Resistance. Microbiology Spectrum, 5.

Ankenbrand, M.J. \& Keller, A. (2016). bcgTree: automatized phylogenetic tree building from bacterial core genomes. Genome, 59, 783-791.

Aurass, P., Düvel, J., Karste, S., Nübel, U., Rabsch, W. \& Flieger, A. (2017). glnA truncation in Salmonella enterica results in a small colony variant phenotype, attenuated host cell entry, and reduced expression of flagellin and SPI-1 associated effector genes. Applied and Environmental Microbiology. 
Avison, M.B., Higgins, C.S., Heldreich, C.J. von, Bennett, P.M. \& Walsh, T.R. (2001). Plasmid Location and Molecular Heterogeneity of the L1 and L2 $\beta$-Lactamase Genes of Stenotrophomonas maltophilia. Antimicrobial Agents and Chemotherapy, 45, 413-419.

Aviv, G., Rahav, G. \& Gal-Mor, O. (2016). Horizontal Transfer of the Salmonella enterica Serovar Infantis Resistance and Virulence Plasmid pESI to the Gut Microbiota of Warm-Blooded Hosts. mBio, 7.

Baker, K.S., Dallman, T.J., Field, N., Childs, T., Mitchell, H., Day, M., Weill, F.-X., Lefèvre, S., Tourdjman, M., Hughes, G., Jenkins, C. \& Thomson, N. (2018). Horizontal antimicrobial resistance transfer drives epidemics of multiple Shigella species. Nature Communications, 9, 1462.

Baltrus, D.A. (2013). Exploring the costs of horizontal gene transfer. Trends in Ecology \& Evolution, 28, 489-495.

Banin, E., Hughes, D. \& Kuipers, O.P. (2017). Editorial: Bacterial pathogens, antibiotics and antibiotic resistance. FEMS Microbiology Reviews, 41, 450-452.

Bankevich, A., Nurk, S., Antipov, D., Gurevich, A.A., Dvorkin, M., Kulikov, A.S., Lesin, V.M., Nikolenko, S.I., Pham, S., Prjibelski, A.D., Pyshkin, A.V., Sirotkin, A.V., Vyahhi, N., Tesler, G., Alekseyev, M.A. \& Pevzner, P.A. (2012). SPAdes: A New Genome Assembly Algorithm and Its Applications to Single-Cell Sequencing. Journal of Computational Biology, 19, 455-477.

Bartolini, A., Frasson, I., Cavallaro, A., Richter, S.N. \& Palù, G. (2014). Comparison of phenotypic methods for the detection of carbapenem non-susceptible Enterobacteriaceae. Gut Pathogens, 6, 13.

Bauernfeind, A., Stemplinger, I., Jungwirth, R. \& Giamarellou, H. (1996). Characterization of the plasmidic beta-lactamase CMY-2, which is responsible for cephamycin resistance. Antimicrobial Agents and Chemotherapy, 40, 221-224.

Ben Said, L., Jouini, A., Alonso, C.A., Klibi, N., Dziri, R., Boudabous, A., Ben Slama, K. $\&$ Torres, C. (2016). Characteristics of extended-spectrum $\beta$-lactamase (ESBL)and pAmpC beta-lactamase-producing Enterobacteriaceae of water samples in Tunisia. Science of The Total Environment, 550, 1103-1109.

Ben-David, D., Kordevani, R., Keller, N., Tal, I., Marzel, A., Gal-Mor, O., Maor, Y. \& Rahav, G. (2012). Outcome of carbapenem resistant Klebsiella pneumoniae bloodstream infections. Clinical Microbiology and Infection, 18, 54-60.

Bengtsson-Palme, J. (2017). Antibiotic resistance in the food supply chain: where can sequencing and metagenomics aid risk assessment? Current Opinion in Food Science, Food Microbiology • Functional Foods and Nutrition, 14, 66-71. 
Bengtsson-Palme, J., Kristiansson, E. \& Larsson, D.G.J. (2018). Environmental factors influencing the development and spread of antibiotic resistance. FEMS Microbiology Reviews, 42.

Bennett, P.M. (2008). Plasmid encoded antibiotic resistance: acquisition and transfer of antibiotic resistance genes in bacteria. British Journal of Pharmacology, 153, S347-S357.

Berendonk, T.U., Manaia, C.M., Merlin, C., Fatta-Kassinos, D., Cytryn, E., Walsh, F., Bürgmann, H., Sørum, H., Norström, M., Pons, M.-N., Kreuzinger, N., Huovinen, P., Stefani, S., Schwartz, T., Kisand, V., Baquero, F. \& Martinez, J.L. (2015). Tackling antibiotic resistance: the environmental framework. Nature Reviews Microbiology, 13, 310.

Bergholz, T.M., Shah, M.K., Burall, L.S., Rakic-Martinez, M. \& Datta, A.R. (2018). Genomic and phenotypic diversity of Listeria monocytogenes clonal complexes associated with human listeriosis. Applied Microbiology and Biotechnology, 102, $3475-3485$.

Bertrand, D., Shaw, J., Kalathiyappan, M., Ng, A.H.Q., Kumar, M.S., Li, C., Dvornicic, M., Soldo, J.P., Koh, J.Y., Tong, C., Ng, O.T., Barkham, T., Young, B., Marimuthu, K., Chng, K.R., Sikic, M. \& Nagarajan, N. (2019). Hybrid metagenomic assembly enables high-resolution analysis of resistance determinants and mobile elements in human microbiomes. Nature Biotechnology, 37, 937-944.

Beyer, P. \& Paulin, S. (2020). Priority pathogens and the antibiotic pipeline: an update. Bulletin of the World Health Organization, 98, 151.

Binh, C.T.T., Heuer, H., Kaupenjohann, M. \& Smalla, K. (2008). Piggery manure used for soil fertilization is a reservoir for transferable antibiotic resistance plasmids. FEMS Microbiology Ecology, 66, 25-37.

Bintsis, T. (2017). Foodborne pathogens. AIMS Microbiology, 3, 529-563.

Blair, J.M.A., Webber, M.A., Baylay, A.J., Ogbolu, D.O. \& Piddock, L.J.V. (2015). Molecular mechanisms of antibiotic resistance. Nature Reviews Microbiology, 13, $42-51$.

Blais, B.W., Gauthier, M., Mohajer, S., Mosher, M. \& Huszczynski, G. (2015). Identification of Listeria monocytogenes colonies by polymerase chain reaction (PCR) and cloth-based hybridization array system (CHAS). In: Compendium of Analytical Methods. Ottawa, Canada: Health Canada.

Böckelmann, U., Dörries, H.-H., Ayuso-Gabella, M.N., Marçay, M.S. de, Tandoi, V., Levantesi, C., Masciopinto, C., Houtte, E.V., Szewzyk, U., Wintgens, T. \& Grohmann, E. (2009). Quantitative PCR Monitoring of Antibiotic Resistance 
Genes and Bacterial Pathogens in Three European Artificial Groundwater Recharge Systems. Applied and Environmental Microbiology, 75, 154-163.

Boerlin, P., Travis, R., Gyles, C.L., Reid-Smith, R., Heather Lim, N.J., Nicholson, V., McEwen, S.A., Friendship, R. \& Archambault, M. (2005). Antimicrobial Resistance and Virulence Genes of Escherichia coli Isolates from Swine in Ontario. Applied and Environmental Microbiology, 71, 6753-6761.

Bogaard, A.E. van den, Bruinsma, N. \& Stobberingh, E.E. (2000). The effect of banning avoparcin on VRE carriage in The Netherlands. Journal of Antimicrobial Chemotherapy, 46, 146-148.

Bogaerts, P., Castro, R.R. de, Mendonça, R. de, Huang, T.-D., Denis, O. \& Glupczynski, Y. (2013). Validation of carbapenemase and extended-spectrum $\beta$-lactamase multiplex endpoint PCR assays according to ISO 15189. Journal of Antimicrobial Chemotherapy, 68, 1576-1582.

Bonnet, R. (2004). Growing Group of Extended-Spectrum $\beta$-Lactamases: the CTX-M Enzymes. Antimicrobial Agents and Chemotherapy, 48, 1-14.

Bonnin, R.A., Nordmann, P., Potron, A., Lecuyer, H., Zahar, J.-R. \& Poirel, L. (2011). Carbapenem-Hydrolyzing GES-Type Extended-Spectrum $\beta$-Lactamase in Acinetobacter baumannii. Antimicrobial Agents and Chemotherapy, 55, 349-354.

Boolchandani, M., D’Souza, A.W. \& Dantas, G. (2019). Sequencing-based methods and resources to study antimicrobial resistance. Nature Reviews Genetics, 20, 356370.

Bopp, D.J., Baker, D.J., Thompson, L., Saylors, A., Root, T.P., Armstrong, L., Mitchell, K., Dumas, N.B. \& Musser, K.A. (2016). Implementation of Salmonella serotype determination using pulsed-field gel electrophoresis in a state public health laboratory. Diagnostic Microbiology and Infectious Disease, 85, 416-418.

Borgianni, L., Luca, F.D., Thaller, M.C., Chong, Y., Rossolini, G.M. \& Docquier, J.-D. (2015). Biochemical Characterization of the POM-1 Metallo- $\beta$-Lactamase from Pseudomonas otitidis. Antimicrobial Agents and Chemotherapy, 59, 1755-1758.

Bottery, M.J., Wood, A.J. \& Brockhurst, M.A. (2016). Selective Conditions for a Multidrug Resistance Plasmid Depend on the Sociality of Antibiotic Resistance. Antimicrobial Agents and Chemotherapy, 60, 2524-2527.

Boyd, D.A., Mataseje, L.F., Davidson, R., Delport, J.A., Fuller, J., Hoang, L., Lefebvre, B., Levett, P.N., Roscoe, D.L., Willey, B.M. \& Mulvey, M.R. (2017). Enterobacter cloacae Complex Isolates Harboring blaNMC-A or blaIMI-Type Class A Carbapenemase Genes on Novel Chromosomal Integrative Elements and Plasmids. Antimicrobial Agents and Chemotherapy, 61, e2578-16. 
Bradley, P., Gordon, N.C., Walker, T.M., Dunn, L., Heys, S., Huang, B., Earle, S., Pankhurst, L.J., Anson, L., Cesare, M. de, Piazza, P., Votintseva, A.A., Golubchik, T., Wilson, D.J., Wyllie, D.H., Diel, R., Niemann, S., Feuerriegel, S., Kohl, T.A., Ismail, N., Omar, S.V., Smith, E.G., Buck, D., McVean, G., Walker, A.S., Peto, T.E.A., Crook, D.W. \& Iqbal, Z. (2015). Rapid antibiotic-resistance predictions from genome sequence data for Staphylococcus aureus and Mycobacterium tuberculosis. Nature Communications, 6, 1-15.

Brandt, J. \& Albertsen, M. (2018). Investigation of Detection Limits and the Influence of DNA Extraction and Primer Choice on the Observed Microbial Communities in Drinking Water Samples Using 16S rRNA Gene Amplicon Sequencing. Frontiers in Microbiology, 9.

Breidenstein, E.B.M., Fuente-Núñez, C. de la \& Hancock, R.E.W. (2011). Pseudomonas aeruginosa: all roads lead to resistance. Trends in Microbiology, 19, 419-426.

Bridier, A., Sanchez-Vizuete, P., Guilbaud, M., Piard, J.-C., Naïtali, M. \& Briandet, R. (2015). Biofilm-associated persistence of food-borne pathogens. Food Microbiology, Special Issue on Predictive modelling in food, 45, 167-178.

Brown, E.D. \& Wright, G.D. (2016). Antibacterial drug discovery in the resistance era. Nature, 529, 336-343.

Brown, E.E.F., Cooper, A., Carrillo, C. \& Blais, B. (2019). Selection of MultidrugResistant Bacteria in Medicated Animal Feeds. Frontiers in Microbiology, 10.

Brown Kav, A., Sasson, G., Jami, E., Doron-Faigenboim, A., Benhar, I. \& Mizrahi, I. (2012). Insights into the bovine rumen plasmidome. Proceedings of the National Academy of Sciences of the United States of America, 109, 5452-5457.

Burke, C., Kjelleberg, S. \& Thomas, T. (2009). Selective Extraction of Bacterial DNA from the Surfaces of Macroalgae. Applied and Environmental Microbiology, 75, $252-256$.

Bush, K. (1999). beta-Lactamases of increasing clinical importance. Current Pharmaceutical Design, 5, 839-845.

Bush, K. (2010). Bench-to-bedside review: The role of $\beta$-lactamases in antibioticresistant Gram-negative infections. Critical Care, 14, 224.

Bush, K. \& Jacoby, G.A. (2010). Updated Functional Classification of $\beta$-Lactamases. Antimicrobial Agents and Chemotherapy, 54, 969-976.

Bush, K., Jacoby, G.A. \& Medeiros, A.A. (1995). A functional classification scheme for beta-lactamases and its correlation with molecular structure. Antimicrobial Agents and Chemotherapy, 39, 1211-1233. 
Bush, S.J., Connor, T.R., Peto, T.E.A., Crook, D.W. \& Walker, A.S. (2020). Evaluation of methods for detecting human reads in microbial sequencing datasets. Microbial Genomics, 6.

Bushnell, B. (2014). BBMap: A Fast, Accurate, Splice-Aware Aligner [Internet document] URL https://sourceforge.net/projects/bbmap/. Accessed 27/02/2019.

Butaye, P., Michael, G.B., Schwarz, S., Barrett, T.J., Brisabois, A. \& White, D.G. (2006). The clonal spread of multidrug-resistant non-typhi Salmonella serotypes. Microbes and Infection, 8, 1891-1897.

Cairns, J., Ruokolainen, L., Hultman, J., Tamminen, M., Virta, M. \& Hiltunen, T. (2018). Ecology determines how low antibiotic concentration impacts community composition and horizontal transfer of resistance genes. Communications Biology, $1,1-8$.

Camacho, C., Coulouris, G., Avagyan, V., Ma, N., Papadopoulos, J., Bealer, K. \& Madden, T.L. (2009). BLAST+: architecture and applications. BMC Bioinformatics, 10, 421.

Campanacci, V., Bishop, R.E., Blangy, S., Tegoni, M. \& Cambillau, C. (2006). The membrane bound bacterial lipocalin Blc is a functional dimer with binding preference for lysophospholipids. FEBS Letters, 580, 4877-4883.

Campbell, E.A., Korzheva, N., Mustaev, A., Murakami, K., Nair, S., Goldfarb, A. \& Darst, S.A. (2001). Structural Mechanism for Rifampicin Inhibition of Bacterial RNA Polymerase. Cell, 104, 901-912.

Cano, D.A., Pucciarelli, M.G., Martínez-Moya, M., Casadesús, J. \& García-del Portillo, F. (2003). Selection of Small-Colony Variants of Salmonella enterica Serovar Typhimurium in Nonphagocytic Eucaryotic Cells. Infection and Immunity, 71, 3690-3698.

Cantas, L., Shah, S.Q.A., Cavaco, L.M., Manaia, C.M., Walsh, F., Popowska, M., Garelick, H., Bürgmann, H. \& Sørum, H. (2013). A brief multi-disciplinary review on antimicrobial resistance in medicine and its linkage to the global environmental microbiota. Frontiers in Microbiology, 4, 96.

Carattoli, A. (2003). Plasmid-mediated antimicrobial resistance in Salmonella enterica. Current Issues in Molecular Biology, 5, 113-122.

Carattoli, A., Tosini, F., Giles, W.P., Rupp, M.E., Hinrichs, S.H., Angulo, F.J., Barrett, T.J. \& Fey, P.D. (2002). Characterization of Plasmids Carrying CMY -2 from Expanded-Spectrum Cephalosporin-Resistant Salmonella Strains Isolated in the United States between 1996 and 1998. Antimicrobial Agents and Chemotherapy, 46, 1269-1272. 
Carpentier, B. \& Cerf, O. (2011). Review — Persistence of Listeria monocytogenes in food industry equipment and premises. International Journal of Food Microbiology, 145, 1-8.

Carrillo, C.D., Koziol, A., Vary, N. \& Blais, B.W. (2019). Applications of Genomics in Regulatory Food Safety Testing in Canada. New Insight into Brucella Infection and Foodborne Diseases.

Carrillo, C.D., Koziol, A.G., Mathews, A., Goji, N., Lambert, D., Huszczynski, G., Gauthier, M., Amoako, K. \& Blais, B.W. (2016). Comparative evaluation of genomic and laboratory approaches for determination of Shiga toxin subtypes in Escherichia coli. Journal of Food Protection, 79, 2078-2085.

Carroll, A.C. \& Wong, A. (2018). Plasmid persistence: costs, benefits, and the plasmid paradox. Canadian Journal of Microbiology, 64, 293-304.

Cartwright, E.J., Jackson, K.A., Johnson, S.D., Graves, L.M., Silk, B.J. \& Mahon, B.E. (2013). Listeriosis Outbreaks and Associated Food Vehicles, United States, 19982008. Emerging Infectious Diseases, 19, 1-9.

Castro, J.C., Rodriguez-R, L.M., Harvey, W.T., Weigand, M.R., Hatt, J.K., Carter, M.Q. \& Konstantinidis, K.T. (2018). imGLAD: accurate detection and quantification of target organisms in metagenomes. PeerJ, 6.

CDC. (2016a). Center for Disease Control and Prevention Achievements in Public Health 1900-1999: Control of Infectious Diseases [Internet document] URL http://www.cdc.gov/mmwr/preview/mmwrhtml/mm4829a1.htm. Accessed $10 / 11 / 2016$.

CDC. (2017b). Biggest Threats | Antibiotic/Antimicrobial Resistance | CDC [Internet document] URL https://www.cdc.gov/drugresistance/biggest_threats.html. Accessed 06/04/2017.

CDC. (2019a). Antibiotic Resistance Threats in the United States, 2019. Atlanta, GA: U.S. Department of Health and Human Services, CDC.

CDC. (2019b). About NARMS | NARMS | CDC [Internet document] URL https://www.cdc.gov/narms/about/index.html. Accessed 23/11/2020.

Cejas, D., Vignoli, R., Quinteros, M., Marino, R., Callejo, R., Betancor, L., Gutkind, G.O. \& Radice, M.A. (2014). First detection of CMY-2 plasmid mediated $\beta$ lactamase in Salmonella Heidelberg in South America. Revista Argentina de Microbiología, 46, 30-33.

Centers for Disease Control and Prevention (CDC). (2002). Outbreak of multidrugresistant Salmonella Newport--United States, January-April 2002. MMWR. Morbidity and mortality weekly report, 51, 545-548. 
Chan, K.-G. (2016). Whole-genome sequencing in the prediction of antimicrobial resistance. Expert Review of Anti-infective Therapy, 14, 617-619.

Chang, H.-J., Hsu, P.-C., Yang, C.-C., Kuo, A.-J., Chia, J.-H., Wu, T.-L. \& Lee, M.-H. (2011). Risk factors and outcomes of carbapenem-nonsusceptible Escherichia coli bacteremia: a matched case-control study. Journal of Microbiology, Immunology, and Infection = Wei Mian Yu Gan Ran Za Zhi, 44, 125-130.

Chatterjee, M. (2016). Antibiotic resistance in Pseudomonas aeruginosa and alternative therapeutic options. International Journal of Medical Microbiology, 306, 48-58.

Che, Y., Xia, Y., Liu, L., Li, A.-D., Yang, Y. \& Zhang, T. (2019). Mobile antibiotic resistome in wastewater treatment plants revealed by Nanopore metagenomic sequencing. Microbiome, 7, 44.

Chenia, H.Y. \& Duma, S. (2017). Characterization of virulence, cell surface characteristics and biofilm-forming ability of Aeromonas spp. isolates from fish and sea water. Journal of Fish Diseases, 40, 339-350.

Cherifi, T., Carrillo, C., Lambert, D., Miniaï, I., Quessy, S., Larivière-Gauthier, G., Blais, B. \& Fravalo, P. (2018). Genomic characterization of Listeria monocytogenes isolates reveals that their persistence in a pig slaughterhouse is linked to the presence of benzalkonium chloride resistance genes. BMC microbiology, 18, 220.

Chiu, C.-H., Lee, J.-J., Wang, M.-H. \& Chu, C. (2020). Genetic analysis and plasmidmediated blaCMY-2 in Salmonella and Shigella and the Ceftriaxone Susceptibility regulated by the ISEcp-1 tnpA-blaCMY-2-blc-sugE. Journal of Microbiology, Immunology and Infection.

Chuang, Y.-M., Tseng, S.-P., Teng, L.-J., Ho, Y.-C. \& Hsueh, P.-R. (2006). Emergence of cefotaxime resistance in Citrobacter freundii causing necrotizing fasciitis and osteomyelitis. The Journal of Infection, 53, e161-163.

Chung, Y.J. \& Saier, M.H. (2002). Overexpression of the Escherichia coli sugE Gene Confers Resistance to a Narrow Range of Quaternary Ammonium Compounds. Journal of Bacteriology, 184, 2543-2545.

Clarke, E.L., Taylor, L.J., Zhao, C., Connell, A., Lee, J.-J., Fett, B., Bushman, F.D. \& Bittinger, K. (2019). Sunbeam: an extensible pipeline for analyzing metagenomic sequencing experiments. Microbiome, 7, 46.

Clausen, P.T.L.C., Aarestrup, F.M. \& Lund, O. (2018). Rapid and precise alignment of raw reads against redundant databases with KMA. BMC Bioinformatics, 19, 307.

Clausen, P.T.L.C., Zankari, E., Aarestrup, F.M. \& Lund, O. (2016). Benchmarking of methods for identification of antimicrobial resistance genes in bacterial whole genome data. Journal of Antimicrobial Chemotherapy, 71, 2484-2488. 
Clinical and Laboratory Standards Institute. (2008). Performance Standards for Antimicrobial Disk and Dilution Susceptibility Tests for Bacteria Isolated from Animals; M31-A3, 3rd Edn.

Clinical and Laboratory Standards Institute. (2013). Performance Standards for antimicrobial susceptibility testing; M100-23, 23rd Edn.

Clinical and Laboratory Standards Institute (CLSI). (2020). Performance Standards for Antimicrobial Susceptibility Testing, 30th Edition. Document M100-ED30:2020.

Codjoe, F.S. \& Donkor, E.S. (2017). Carbapenem Resistance: A Review. Medical Sciences, $\mathbf{6}$.

Cogliani, C., Goossens, H. \& Greko, C. (2011). Restricting Antimicrobial Use in Food Animals: Lessons from Europe: Banning nonessential antibiotic uses in food animals is intended to reduce pools of resistance genes. Microbe Magazine, $\mathbf{6}$, 274-279.

Conlan, S., Park, M., Deming, C., Thomas, P.J., Young, A.C., Coleman, H., Sison, C., NISC Comparative Sequencing Program, Weingarten, R.A., Lau, A.F., Dekker, J.P., Palmore, T.N., Frank, K.M. \& Segre, J.A. (2016). Plasmid Dynamics in KPC-Positive Klebsiella pneumoniae during Long-Term Patient Colonization. mBio, 7.

Cooper, A., Koziol, A.G., Carrillo, C.D. \& Lambert, D. (2016). Draft Genome Sequences of Salmonella enterica subsp. enterica Serovar Berta ATCC 8392 and a Nalidixic Acid-Resistant Isolate of This Strain. Genome Announcements, 4.

Cooper, A., Lambert, D., Koziol, A.G., Seyer, K. \& Carrillo, C.D. (2015). Draft Genome Sequence of Salmonella enterica subsp. enterica Serovar Mishmarhaemek Isolated from Bovine Feces. Genome Announc., 3, e01210-15.

Cooper, A.L., Low, A.J., Koziol, A.G., Thomas, M.C., Leclair, D., Tamber, S., Wong, A., Blais, B.W. \& Carrillo, C.D. (2020). Systematic Evaluation of Whole Genome Sequence-Based Predictions of Salmonella Serotype and Antimicrobial Resistance. Frontiers in Microbiology, 11, 549.

Crader, M.F. \& Varacallo, M. (2020). Preoperative Antibiotic Prophylaxis. In: StatPearls. Treasure Island (FL): StatPearls Publishing.

Currie, A., Farber, J.M., Nadon, C., Sharma, D., Whitfield, Y., Gaulin, C., Galanis, E., Bekal, S., Flint, J., Tschetter, L., Pagotto, F., Lee, B., Jamieson, F., Badiani, T., MacDonald, D., Ellis, A., May-Hadford, J., McCormick, R., Savelli, C., Middleton, D., Allen, V., Tremblay, F.-W., MacDougall, L., Hoang, L., Shyng, S., Everett, D., Chui, L., Louie, M., Bangura, H., Levett, P.N., Wilkinson, K., Wylie, J., Reid, J., Major, B., Engel, D., Douey, D., Huszczynski, G., Di Lecci, J., Strazds, J., Rousseau, J., Ma, K., Isaac, L. \& Sierpinska, U. (2015). Multiprovince listeriosis outbreak linked to contaminated deli meat consumed primarily 
in institutional settings, Canada, 2008. Foodborne Pathogens and Disease, 12, $645-652$.

Czajkowski, M.D., Vance, D.P., Frese, S.A. \& Casaburi, G. (2019). GenCoF: a graphical user interface to rapidly remove human genome contaminants from metagenomic datasets. Bioinformatics, 35, 2318-2319.

Davies, J. (2006). Where have All the Antibiotics Gone? The Canadian Journal of Infectious Diseases \& Medical Microbiology, 17, 287-290.

Davison, J. (1999). Genetic exchange between bacteria in the environment. Plasmid, 42, 73-91.

Denisuik, A.J., Garbutt, L.A., Golden, A.R., Adam, H.J., Baxter, M., Nichol, K.A., Lagacé-Wiens, P., Walkty, A.J., Karlowsky, J.A., Hoban, D.J., Mulvey, M.R. \& Zhanel, G.G. (2019a). Antimicrobial-resistant pathogens in Canadian ICUs: results of the CANWARD 2007 to 2016 study. Journal of Antimicrobial Chemotherapy, 74, 645-653.

Denisuik, A.J., Karlowsky, J.A., Adam, H.J., Baxter, M.R., Lagacé-Wiens, P.R.S., Mulvey, M.R., Hoban, D.J., Zhanel, G.G., Canward, C.A.R.A. (CARA) and, Zhanel, G.G., Hoban, D.J., Adam, H.J., Baxter, M.R., Nichol, K.A., LagacéWiens, P.R.S., Walkty, A., Karlowsky, J.A., Blondeau, J., Slinger, R., Davidson, R., Zhanel, G., Hoban, D., Delport, J., Ellis, C., Laverdière, M., Loo, V., Poutanen, S., Fuller, J., Roscoe, D., Desjardins, M., Matukas, L., Goyette, M., Lee, C., Carignan, A., Bergevin, M. \& Pelletier, R. (2019b). Dramatic rise in the proportion of ESBL-producing Escherichia coli and Klebsiella pneumoniae among clinical isolates identified in Canadian hospital laboratories from 2007 to 2016. Journal of Antimicrobial Chemotherapy, 74, iv64-iv71.

Di Tommaso, P., Moretti, S., Xenarios, I., Orobitg, M., Montanyola, A., Chang, J.-M., Taly, J.-F. \& Notredame, C. (2011). T-Coffee: a web server for the multiple sequence alignment of protein and RNA sequences using structural information and homology extension. Nucleic Acids Research, 39, W13-17.

Dib, J.R., Wagenknecht, M., Farías, M.E. \& Meinhardt, F. (2015). Strategies and approaches in plasmidome studies - uncovering plasmid diversity disregarding of linear elements? Frontiers in Microbiology, 6.

Diene, S.M. \& Rolain, J.-M. (2014). Carbapenemase genes and genetic platforms in Gram-negative bacilli: Enterobacteriaceae, Pseudomonas and Acinetobacter species. Clinical Microbiology and Infection, 20, 831-838.

Dierikx, C.M., Goot, J.A. van der, Smith, H.E., Kant, A. \& Mevius, D.J. (2013). Presence of ESBL/AmpC -Producing Escherichia coli in the Broiler Production Pyramid: A Descriptive Study. PLOS ONE, 8, e79005. 
Dimitriu, T., Marchant, L., Buckling, A. \& Raymond, B. (2019). Bacteria from natural populations transfer plasmids mostly towards their kin. Proceedings of the Royal Society B: Biological Sciences, 286.

Dimitriu, T., Misevic, D., Lotton, C., Brown, S.P., Lindner, A.B. \& Taddei, F. (2016). Indirect Fitness Benefits Enable the Spread of Host Genes Promoting Costly Transfer of Beneficial Plasmids. PLoS biology, 14, e1002478.

Doi, Y., Paterson, D.L., Egea, P., Pascual, A., López-Cerero, L., Navarro, M.D., AdamsHaduch, J.M., Qureshi, Z.A., Sidjabat, H.E. \& Rodríguez-Baño, J. (2010). Extended-spectrum and CMY-type b-lactamase-producing Escherichia coli in clinical samples and retail meat from Pittsburgh, USA and Seville, Spain. Clinical Microbiology and Infection, 16, 33-38.

Drouin, A., Letarte, G., Raymond, F., Marchand, M., Corbeil, J. \& Laviolette, F. (2019). Interpretable genotype-to-phenotype classifiers with performance guarantees. Scientific Reports, 9, 4071.

Duffy, B., Holliger, E. \& Walsh, F. (2014). Streptomycin use in apple orchards did not increase abundance of mobile resistance genes. FEMS Microbiology Letters, 350, $180-189$.

Duin, D. van \& Doi, Y. (2016). The global epidemiology of carbapenemase-producing Enterobacteriaceae. Virulence, 8, 460-469.

Dutil, L., Irwin, R., Finley, R., Ng, L.K., Avery, B., Boerlin, P., Bourgault, A.M., Cole, L., Daignault, D., Desruisseau, A., Demczuk, W., Hoang, L., Horsman, G.B., Ismail, J., Jamieson, F., Maki, A., Pacagnella, A. \& Pillai, D.R. (2010). Ceftiofur resistance in Salmonella enterica serovar Heidelberg from chicken meat and humans, Canada. Emerging Infectious Diseases, 16, 48-54.

Dutta, V., Elhanafi, D. \& Kathariou, S. (2013). Conservation and distribution of the benzalkonium chloride resistance cassette bcrABC in Listeria monocytogenes. Applied and Environmental Microbiology, 79, 6067-6074.

EARS-Net. (2020). European Antimicrobial Resistance Surveillance Network (EARSNet) [Internet document]. European Centre for Disease Prevention and Control. URL https://www.ecdc.europa.eu/en/about-us/partnerships-and-networks/diseaseand-laboratory-networks/ears-net. Accessed 23/11/2020.

Edirmanasinghe, R., Finley, R., Parmley, E.J., Avery, B.P., Carson, C., Bekal, S., Golding, G. \& Mulvey, M.R. (2017). A whole-genome sequencing approach to study cefoxitin-resistant Salmonella enterica serovar Heidelberg isolates from various sources. Antimicrobial Agents and Chemotherapy, 61, e01919-16.

Elhanafi, D., Dutta, V. \& Kathariou, S. (2010). Genetic characterization of plasmidassociated benzalkonium chloride resistance determinants in a Listeria 
monocytogenes strain from the 1998-1999 outbreak. Applied and Environmental Microbiology, 76, 8231-8238.

Esterly, J.S., Wagner, J., McLaughlin, M.M., Postelnick, M.J., Qi, C. \& Scheetz, M.H. (2012). Evaluation of Clinical Outcomes in Patients with Bloodstream Infections Due to Gram-Negative Bacteria According to Carbapenem MIC Stratification. Antimicrobial Agents and Chemotherapy, 56, 4885-4890.

EUCAST. (2020). The European Committee on Antimicrobial Susceptibility Testing. Breakpoint tables for interpretation of MICs and zone diameters. Version 10.0.

Feldgarden, M., Brover, V., Haft, D.H., Prasad, A.B., Slotta, D.J., Tolstoy, I., Tyson, G.H., Zhao, S., Hsu, C.-H., McDermott, P.F., Tadesse, D.A., Morales, C., Simmons, M., Tillman, G., Wasilenko, J., Folster, J.P. \& Klimke, W. (2019). Validating the NCBI AMRFinder Tool and Resistance Gene Database Using Antimicrobial Resistance Genotype-Phenotype Correlations in a Collection of NARMS Isolates. Antimicrobial Agents and Chemotherapy, AAC.00483-19.

Fernández Márquez, M.L., Burgos, M.J.G., Pulido, R.P., Gálvez, A. \& López, R.L. (2016). Biocide Tolerance and Antibiotic Resistance in Salmonella Isolates from Hen Eggshells. Foodborne Pathogens and Disease, 14, 89-95.

Ferreira, V., Wiedmann, M., Teixeira, P. \& Stasiewicz, M.J. (2014). Listeria monocytogenes persistence in food-associated environments: epidemiology, strain characteristics, and implications for public health. Journal of Food Protection, 77, $150-170$.

Fevre, C., Jbel, M., Passet, V., Weill, F.-X., Grimont, P.A.D. \& Brisse, S. (2005). Six Groups of the OXY $\beta$-Lactamase Evolved over Millions of Years in Klebsiella oxytoca. Antimicrobial Agents and Chemotherapy, 49, 3453-3462.

Figueira, V., Vaz-Moreira, I., Silva, M. \& Manaia, C.M. (2011). Diversity and antibiotic resistance of Aeromonas spp. in drinking and wastewater treatment plants. Water Research, 45, 5599-5611.

Fitzpatrick, D. \& Walsh, F. (2016). Antibiotic resistance genes across a wide variety of metagenomes. FEMS Microbiology Ecology, 92.

Fluit, A.C., Visser, M.R. \& Schmitz, F.-J. (2001). Molecular Detection of Antimicrobial Resistance. Clinical Microbiology Reviews, 14, 836-871.

Folster, J.P., Pecic, G., McCullough, A., Rickert, R. \& Whichard, J.M. (2011). Characterization of blaCMY-Encoding Plasmids Among Salmonella Isolated in the United States in 2007. Foodborne Pathogens and Disease, 8, 1289-1294.

Forward, K.R., Matheson, K.M., Hiltz, M., Musgrave, H. \& Poppe, C. (2004). Recovery of Cephalosporin Resistant Escherichia coli and Salmonella from Pork, Beef and 
Chicken Marketed in Nova Scotia. Canadian Journal of Infectious Diseases and Medical Microbiology, 4, 226-230.

Franiczek, R., Krzyżanowska, B., Dolna, I. \& Mokracka-Latajka, G. (2010). Conjugative Transfer of Plasmid-Mediated CTX-M-Type $\beta$-Lactamases from Clinical Strains of Enterobacteriaceae to Salmonella enterica Serovars. Advances in Clinical and Experimental Medicine, 19, 313-322.

Freeman, C.N., Scriver, L., Neudorf, K.D., Hansen, L.T., Jamieson, R.C. \& Yost, C.K. (2018). Antimicrobial resistance gene surveillance in the receiving waters of an upgraded wastewater treatment plant. FACETS.

Futoma-Kołoch, B., Książczyk, M., Korzekwa, K., Migdał, I., Pawlak, A., Jankowska, M., Kędziora, A., Dorotkiewicz-Jach, A. \& Bugla-Płoskońska, G. (2015). Selection and electrophoretic characterization of Salmonella enterica subsp. enterica biocide variants resistant to antibiotics. Polish Journal of Veterinary Sciences, 18, 725-732.

Gabor, E.M., Vries, E.J. de \& Janssen, D.B. (2003). Efficient recovery of environmental DNA for expression cloning by indirect extraction methods. FEMS Microbiology Ecology, 44, 153-163.

Gama, J.A., Zilhão, R. \& Dionisio, F. (2020). Plasmid Interactions Can Improve Plasmid Persistence in Bacterial Populations. Frontiers in Microbiology, 11.

Gantzhorn, M.R., Pedersen, K., Olsen, J.E. \& Thomsen, L.E. (2014). Biocide and antibiotic susceptibility of Salmonella isolates obtained before and after cleaning at six Danish pig slaughterhouses. International Journal of Food Microbiology, 181, 53-59.

García-Alcalde, F., Okonechnikov, K., Carbonell, J., Cruz, L.M., Götz, S., Tarazona, S., Dopazo, J., Meyer, T.F. \& Conesa, A. (2012). Qualimap: evaluating nextgeneration sequencing alignment data. Bioinformatics, 28, 2678-2679.

Gargis, A.S., Kalman, L., Berry, M.W., Bick, D.P., Dimmock, D.P., Hambuch, T., Lu, F., Lyon, E., Voelkerding, K.V., Zehnbauer, B.A., Agarwala, R., Bennett, S.F., Chen, B., Chin, E.L.H., Compton, J.G., Das, S., Farkas, D.H., Ferber, M.J., Funke, B.H., Furtado, M.R., Ganova-Raeva, L.M., Geigenmüller, U., Gunselman, S.J., Hegde, M.R., Johnson, P.L.F., Kasarskis, A., Kulkarni, S., Lenk, T., Liu, C.S.J., Manion, M., Manolio, T.A., Mardis, E.R., Merker, J.D., Rajeevan, M.S., Reese, M.G., Rehm, H.L., Simen, B.B., Yeakley, J.M., Zook, J.M. \& Lubin, I.M. (2012). Assuring the quality of next-generation sequencing in clinical laboratory practice. Nature Biotechnology, 30, 1033-1036.

Garneau-Tsodikova, S. \& Labby, K.J. (2016). Mechanisms of Resistance to Aminoglycoside Antibiotics: Overview and Perspectives. MedChemComm, 7, 1127. 
Garner, D. \& Kathariou, S. (2016). Fresh Produce-Associated Listeriosis Outbreaks, Sources of Concern, Teachable Moments, and Insights. Journal of Food Protection, 79, 337-344.

Gaudin, M. \& Desnues, C. (2018). Hybrid Capture-Based Next Generation Sequencing and Its Application to Human Infectious Diseases. Frontiers in Microbiology, 9.

Gaulin, C., Ramsay, D. \& Bekal, S. (2012). Widespread listeriosis outbreak attributable to pasteurized cheese, which led to extensive cross-contamination affecting cheese retailers, Quebec, Canada, 2008. Journal of Food Protection, 75, 71-78.

Geneva: World Health Organization. (2019). 2019 Antibacterial agents in clinical development: an analysis of the antibacterial clinical development pipeline.

Gieraltowski, L., Higa, J., Peralta, V., Green, A., Schwensohn, C., Rosen, H., Libby, T., Kissler, B., Marsden-Haug, N., Booth, H., Kimura, A., Grass, J., Bicknese, A., Tolar, B., Defibaugh-Chávez, S., Williams, I., Wise, M. \& Team, S.H.I. (2016). National Outbreak of Multidrug Resistant Salmonella Heidelberg Infections Linked to a Single Poultry Company. PLoS ONE, 11.

Gilmour, M.W., Graham, M., Van Domselaar, G., Tyler, S., Kent, H., Trout-Yakel, K.M., Larios, O., Allen, V., Lee, B. \& Nadon, C. (2010). High-throughput genome sequencing of two Listeria monocytogenes clinical isolates during a large foodborne outbreak. BMC Genomics, 11, 120.

Girlich, D., Naas, T., Bellais, S., Poirel, L., Karim, A. \& Nordmann, P. (2000). Heterogeneity of AmpC Cephalosporinases of Hafnia alvei Clinical Isolates Expressing Inducible or Constitutive Ceftazidime Resistance Phenotypes. Antimicrobial Agents and Chemotherapy, 44, 3220-3223.

Girlich, D., Poirel, L. \& Nordmann, P. (2012). Value of the Modified Hodge Test for Detection of Emerging Carbapenemases in Enterobacteriaceae. Journal of Clinical Microbiology, 50, 477-479.

Gombas, D.E., Chen, Y., Clavero, R.S. \& Scott, V.N. (2003). Survey of Listeria monocytogenes in Ready-to-Eat Foods. Journal of Food Protection, 66, 559-569.

Gonzalez Leiza, M., Perez-Diaz, J.C., Ayala, J., Casellas, J.M., Martinez-Beltran, J., Bush, K. \& Baquero, F. (1994). Gene sequence and biochemical characterization of FOX-1 from Klebsiella pneumoniae, a new AmpC-type plasmid-mediated beta-lactamase with two molecular variants. Antimicrobial Agents and Chemotherapy, 38, 2150-2157.

Gould, K. (2016). Antibiotics: from prehistory to the present day. Journal of Antimicrobial Chemotherapy, 71, 572-575. 
Government of Canada. (2009a). Report of the Independent Investigator into the 2008 listeriosis outbreak [Internet document] URL

http://publications.gc.ca/site/eng/361474/publication.html. Accessed 13/08/2020.

Government of Canada. (2013). Canadian Integrated Program for Antimicrobial Resistance Surveillance (CIPARS) 2013 Annual Report - Chapter 2.

Antimicrobial Resistance. Public Health Agency of Canada, Guelph, Ontario, 2015.

Government of Canada, C.F.I.A. (2016). National Microbiological Baseline Study in Broiler Chicken December 2012 - December 2013 [Internet document] URL http://www.inspection.gc.ca/food/chemical-residues-microbiology/food-safetytesting-bulletins/2016-08-17/december-2012-december2013/eng/1471358115567/1471358175297. Accessed 07/02/2019.

Government of Canada, H.C. (2009b). Categorization of Antimicrobial Drugs Based on Importance in Human Medicine - Veterinary Drugs - Health Canada [Internet document] URL http://www.hc-sc.gc.ca/dhp-mps/vet/antimicrob/amr_ram_hummed-rev-eng.php. Accessed 16/02/2017.

Government of Canada, P.H.A. of C. (2007). Canadian Integrated Program for Antimicrobial Resistance Surveillance (CIPARS) - Public Health Agency Canada [Internet document] URL http://www.phac-aspc.gc.ca/cipars-picra/index-eng.php. Accessed 11/10/2016.

Government of Canada, P.H.A. of C. (2020). Canadian Antimicrobial Resistance Surveillance System - Update 2020. Public Health Agency of Canada.

Grimont, P. \& Weill, F. (2007). Antigenic formulae of the Salmonella serovars (9th ed.), WHO Collaborating Center for Reference and Research on Salmonella. In: Antigenic formulae of the Salmonella serovars. 9th ed [Internet]. Presented at the WHO Collaborating Centre for Reference and Research on Salmonella. Institut Pasteur, Paris, France.

Grüning, B., Dale, R., Sjödin, A., Chapman, B.A., Rowe, J., Tomkins-Tinch, C.H., Valieris, R. \& Köster, J. (2018). Bioconda: sustainable and comprehensive software distribution for the life sciences. Nature Methods, 15, 475.

Guitor, A.K., Raphenya, A.R., Klunk, J., Kuch, M., Alcock, B., Surette, M.G., McArthur, A.G., Poinar, H.N. \& Wright, G.D. (2019). Capturing the Resistome: a Targeted Capture Method To Reveal Antibiotic Resistance Determinants in Metagenomes. Antimicrobial Agents and Chemotherapy, 64.

Guo, Y.-F., Zhang, W.-H., Ren, S.-Q., Yang, L., Lü, D.-H., Zeng, Z.-L., Liu, Y.-H. \& Jiang, H.-X. (2014). IncA/C Plasmid-Mediated Spread of CMY-2 in MultidrugResistant Escherichia coli from Food Animals in China. PLOS ONE, 9, e96738. 
Gupta, P., Bhatia, M., Gupta, P. \& Omar, B.J. (2018). Emerging Biocide Resistance among Multidrug-Resistant Bacteria: Myth or Reality? A Pilot Study. Journal of Pharmacy \& Bioallied Sciences, 10, 96-101.

Gupta, S.K., Padmanabhan, B.R., Diene, S.M., Lopez-Rojas, R., Kempf, M., Landraud, L. \& Rolain, J.-M. (2014). ARG-ANNOT, a New Bioinformatic Tool To Discover Antibiotic Resistance Genes in Bacterial Genomes. Antimicrobial Agents and Chemotherapy, 58, 212-220.

Gurevich, A., Saveliev, V., Vyahhi, N. \& Tesler, G. (2013). QUAST: quality assessment tool for genome assemblies. Bioinformatics, 29, 1072-1075.

Haenni, M., Saras, E., Métayer, V., Médaille, C. \& Madec, J.-Y. (2014). High Prevalence of blaCTX-M-1/IncI1/ST3 and blaCMY-2/IncI1/ST2 Plasmids in Healthy Urban Dogs in France. Antimicrobial Agents and Chemotherapy, 58, 5358-5362.

Haft, R.J.F., Mittler, J.E. \& Traxler, B. (2009). Competition favours reduced cost of plasmids to host bacteria. The ISME Journal, 3, 761-769.

Hakanen, A., Kotilainen, P., Jalava, J., Siitonen, A. \& Huovinen, P. (1999). Detection of Decreased Fluoroquinolone Susceptibility in Salmonellas and Validation of Nalidixic Acid Screening Test. Journal of Clinical Microbiology, 37, 3572-3577.

Hao, M., Ye, M., Shen, Z., Hu, F., Yang, Y., Wu, S., Xu, X., Zhu, S., Qin, X. \& Wang, M. (2018). Porin Deficiency in Carbapenem-Resistant Enterobacter aerogenes Strains. Microbial Drug Resistance, 24, 1277-1283.

Haque, M.M., Bose, T., Dutta, A., Reddy, C.V.S.K. \& Mande, S.S. (2015). CS-SCORE: Rapid identification and removal of human genome contaminants from metagenomic datasets. Genomics, 106, 116-121.

Hardiman, C.A., Weingarten, R.A., Conlan, S., Khil, P., Dekker, J.P., Mathers, A.J., Sheppard, A.E., Segre, J.A. \& Frank, K.M. (2016). Horizontal Transfer of Carbapenemase-Encoding Plasmids and Comparison with Hospital Epidemiology Data. Antimicrobial Agents and Chemotherapy, 60, 4910-4919.

Harrand, A.S., Jagadeesan, B., Baert, L., Wiedmann, M. \& Orsi, R.H. (2020). Evolution of Listeria monocytogenes in a food-processing plant involves limited single nucleotide substitutions, but considerable diversification by gain and loss of prophages. Applied and Environmental Microbiology, 86, e02493-19.

Harrison, E. \& Brockhurst, M.A. (2012). Plasmid-mediated horizontal gene transfer is a coevolutionary process. Trends in Microbiology, 20, 262-267.

Harrison, E., Guymer, D., Spiers, A.J., Paterson, S. \& Brockhurst, M.A. (2015). Parallel compensatory evolution stabilizes plasmids across the parasitism-mutualism continuum. Current biology: CB, 25, 2034-2039. 
Hasan, M.R., Rawat, A., Tang, P., Jithesh, P.V., Thomas, E., Tan, R. \& Tilley, P. (2016). Depletion of Human DNA in Spiked Clinical Specimens for Improvement of Sensitivity of Pathogen Detection by Next-Generation Sequencing. Journal of Clinical Microbiology, 54, 919-927.

Hasman, H. \& Aarestrup, F.M. (2002). tcrB, a Gene Conferring Transferable Copper Resistance in Enterococcus faecium: Occurrence, Transferability, and Linkage to Macrolide and Glycopeptide Resistance. Antimicrobial Agents and Chemotherapy, 46, 1410-1416.

Haubert, L., Zehetmeyr, M.L. \& Silva, W.P. da. (2019). Resistance to benzalkonium chloride and cadmium chloride in Listeria monocytogenes isolates from food and food-processing environments in southern Brazil. Canadian Journal of Microbiology, 65, 429-435.

Helmy, M.M. \& Wasfi, R. (2014). Phenotypic and Molecular Characterization of Plasmid Mediated AmpC $\beta$-Lactamases among Escherichia coli, Klebsiella spp., and Proteus mirabilis Isolated from Urinary Tract Infections in Egyptian Hospitals. BioMed Research International.

Hembach, N., Schmid, F., Alexander, J., Hiller, C., Rogall, E.T. \& Schwartz, T. (2017). Occurrence of the mcr- 1 Colistin Resistance Gene and other Clinically Relevant Antibiotic Resistance Genes in Microbial Populations at Different Municipal Wastewater Treatment Plants in Germany. Frontiers in Microbiology, 8.

Heuer, H. \& Smalla, K. (2012). Plasmids foster diversification and adaptation of bacterial populations in soil. FEMS Microbiology Reviews, 36, 1083-1104.

Hoek, A.H.A.M. van, Mevius, D., Guerra, B., Mullany, P., Roberts, A.P. \& Aarts, H.J.M. (2011). Acquired antibiotic resistance genes: an overview. Antimicrobials, Resistance and Chemotherapy, 2, 203.

Högenauer, C., Langner, C., Beubler, E., Lippe, I.T., Schicho, R., Gorkiewicz, G., Krause, R., Gerstgrasser, N., Krejs, G.J. \& Hinterleitner, T.A. (2006). Klebsiella oxytoca as a Causative Organism of Antibiotic-Associated Hemorrhagic Colitis. New England Journal of Medicine, 355, 2418-2426.

Holah, J.T., Taylor, J.H., Dawson, D.J. \& Hall, K.E. (2002). Biocide use in the food industry and the disinfectant resistance of persistent strains of Listeria monocytogenes and Escherichia coli. Journal of Applied Microbiology, 92, 111S$120 \mathrm{~S}$.

Holmberg, S.D., Osterholm, M.T., Senger, K.A. \& Cohen, M.L. (1984). Drug-resistant Salmonella from animals fed antimicrobials. The New England Journal of Medicine, 311, 617-622. 
Holmes, A.H., Moore, L.S.P., Sundsfjord, A., Steinbakk, M., Regmi, S., Karkey, A., Guerin, P.J. \& Piddock, L.J.V. (2016). Understanding the mechanisms and drivers of antimicrobial resistance. The Lancet, 387, 176-187.

Hong, S., Rovira, A., Davies, P., Ahlstrom, C., Muellner, P., Rendahl, A., Olsen, K., Bender, J.B., Wells, S., Perez, A. \& Alvarez, J. (2016). Serotypes and Antimicrobial Resistance in Salmonella enterica Recovered from Clinical Samples from Cattle and Swine in Minnesota, 2006 to 2015. PLoS ONE, 11, e0168016.

Hopkins, D.W., Macnaughton, S.J. \& O’Donnell, A.G. (n.d.). A dispersion and differential centrifugation technique for representatively sampling microorganisms from soil. Soil Biology and Biochemistry, 23, 217-225.

Hu, Y., Cai, J., Zhang, R., Zhou, H., Sun, Q. \& Chen, G. (2012). Emergence of Proteus mirabilis Harboring blaKPC-2 and $q n r D$ in a Chinese Hospital. Antimicrobial Agents and Chemotherapy, 56, 2278-2282.

Hudson, J.A., Frewer, L.J., Jones, G., Brereton, P.A., Whittingham, M.J. \& Stewart, G. (2017). The agri-food chain and antimicrobial resistance: A review. Trends in Food Science \& Technology, 69, 131-147.

Hug, L.A. (2018). Sizing Up the Uncultured Microbial Majority. mSystems, 3.

Hugerth, L.W. \& Andersson, A.F. (2017). Analysing Microbial Community Composition through Amplicon Sequencing: From Sampling to Hypothesis Testing. Frontiers in Microbiology, $\mathbf{8}$.

Huijbers, P.M.C., Blaak, H., Jong, M.C.M. de, Graat, E.A.M., Vandenbroucke-Grauls, C.M.J.E. \& Roda Husman, A.M. de. (2015). Role of the Environment in the Transmission of Antimicrobial Resistance to Humans: A Review. Environmental Science \& Technology, 49, 11993-12004.

Hutchings, M.I., Truman, A.W. \& Wilkinson, B. (2019). Antibiotics: past, present and future. Current Opinion in Microbiology, Antimicrobials, 51, 72-80.

Hutinel, M., Huijbers, P.M.C., Fick, J., Åhrén, C., Larsson, D.G.J. \& Flach, C.-F. (2019). Population-level surveillance of antibiotic resistance in Escherichia coli through sewage analysis. Eurosurveillance, 24, 1800497.

Ibrahimagić, A., Bedenić, B., Kamberović, F. \& Uzunović, S. (2015). High prevalence of CTX-M-15 and first report of CTX-M-3, CTX-M-22, CTX-M-28 and plasmidmediated AmpC beta-lactamase producing Enterobacteriaceae causing urinary tract infections in Bosnia and Herzegovina in hospital and community settings. Journal of Infection and Chemotherapy, 21, 363-369. 
Inouye, M., Dashnow, H., Raven, L.-A., Schultz, M.B., Pope, B.J., Tomita, T., Zobel, J. \& Holt, K.E. (2014). SRST2: Rapid genomic surveillance for public health and hospital microbiology labs. Genome Medicine, 6, 90.

Jacoby, G.A. (2009). AmpC $\beta$-Lactamases. Clinical Microbiology Reviews, 22, 161-182.

Jacoby, G.A., Mills, D.M. \& Chow, N. (2004). Role of beta-lactamases and porins in resistance to ertapenem and other beta-lactams in Klebsiella pneumoniae.

Antimicrobial Agents and Chemotherapy, 48, 3203-3206.

Jain, A. \& Srivastava, P. (2013). Broad host range plasmids. FEMS Microbiology Letters, 348, 87-96.

Jernberg, C., Löfmark, S., Edlund, C. \& Jansson, J.K. (2010). Long-term impacts of antibiotic exposure on the human intestinal microbiota. Microbiology, 156, 32163223 .

Jiang, X., Yu, T., Liang, Y., Ji, S., Guo, X., Ma, J. \& Zhou, L. (2016). Efflux pumpmediated benzalkonium chloride resistance in Listeria monocytogenes isolated from retail food. International Journal of Food Microbiology, 217, 141-145.

Johansen, S.K., Maus, C.E., Plikaytis, B.B. \& Douthwaite, S. (2006). Capreomycin Binds across the Ribosomal Subunit Interface Using tlyA-Encoded 2'-O-Methylations in 16S and 23S rRNAs. Molecular Cell, 23, 173-182.

Johnson, J.S., Spakowicz, D.J., Hong, B.-Y., Petersen, L.M., Demkowicz, P., Chen, L., Leopold, S.R., Hanson, B.M., Agresta, H.O., Gerstein, M., Sodergren, E. \& Weinstock, G.M. (2019). Evaluation of 16S rRNA gene sequencing for species and strain-level microbiome analysis. Nature Communications, 10, 5029.

Jolley, K.A., Bliss, C.M., Bennett, J.S., Bratcher, H.B., Brehony, C., Colles, F.M., Wimalarathna, H., Harrison, O.B., Sheppard, S.K., Cody, A.J. \& Maiden, M.C.J. (2012). Ribosomal multilocus sequence typing: universal characterization of bacteria from domain to strain. Microbiology (Reading, England), 158, 10051015 .

Jousset, A.B., Oueslati, S., Bernabeu, S., Takissian, J., Creton, E., Vogel, A., Sauvadet, A., Cotellon, G., Gauthier, L., Bonnin, R.A., Dortet, L. \& Naas, T. (2019). FalsePositive Carbapenem-Hydrolyzing Confirmatory Tests Due to ACT-28, a Chromosomally Encoded AmpC with Weak Carbapenemase Activity from Enterobacter kobei. Antimicrobial Agents and Chemotherapy, 63.

Jutkina, J., Marathe, N.P., Flach, C.-F. \& Larsson, D.G.J. (2018). Antibiotics and common antibacterial biocides stimulate horizontal transfer of resistance at low concentrations. Science of The Total Environment, 616-617, 172-178.

Kalkut, G. (1998). Sulfonamides and Trimethoprim. Cancer Investigation, 16, 612-615. 
Kameyama, M., Chuma, T., Yabata, J., Tominaga, K., Iwata, H. \& Okamoto, K. (2013). Prevalence and epidemiological relationship of CMY-2 AmpC $\beta$-lactamase and CTX-M extended-spectrum $\beta$-lactamase-producing Escherichia coli isolates from broiler farms in Japan. The Journal of Veterinary Medical Science, 75, 10091015.

Kapoor, G., Saigal, S. \& Elongavan, A. (2017). Action and resistance mechanisms of antibiotics: A guide for clinicians. Journal of Anaesthesiology, Clinical Pharmacology, 33, 300-305.

Katharios-Lanwermeyer, S., Rakic-Martinez, M., Elhanafi, D., Ratani, S., Tiedje, J.M. \& Kathariou, S. (2012). Co-selection of cadmium and benzalkonium chloride resistance in conjugative transfers from nonpathogenic Listeria spp. to other Listeriae. Applied and Environmental Microbiology, 78, 7549-7556.

Keegan, K.P., Glass, E.M. \& Meyer, F. (2016). MG-RAST, a Metagenomics Service for Analysis of Microbial Community Structure and Function. Methods in Molecular Biology (Clifton, N.J.), 1399, 207-233.

Kim, S.-H., Wei, C.-I. \& An, H. (2005). Molecular Characterization of MultidrugResistant Proteus mirabilis Isolates from Retail Meat Products. Journal of Food Protection, 68, 1408-1413.

Kimera, Z.I., Mshana, S.E., Rweyemamu, M.M., Mboera, L.E.G. \& Matee, M.I.N. (2020). Antimicrobial use and resistance in food-producing animals and the environment: an African perspective. Antimicrobial Resistance \& Infection Control, 9, 37.

Kirchhelle, C. (2018). Pharming animals: a global history of antibiotics in food production (1935-2017). Palgrave Communications, 4, 1-13.

Kittinger, C., Lipp, M., Baumert, R., Folli, B., Koraimann, G., Toplitsch, D., Liebmann, A., Grisold, A.J., Farnleitner, A.H., Kirschner, A. \& Zarfel, G. (2016). Antibiotic Resistance Patterns of Pseudomonas spp. Isolated from the River Danube. Frontiers in Microbiology, 7.

Klümper, U., Riber, L., Dechesne, A., Sannazzarro, A., Hansen, L.H., Sørensen, S.J. \& Smets, B.F. (2015). Broad host range plasmids can invade an unexpectedly diverse fraction of a soil bacterial community. The ISME journal, 9, 934-945.

Knabel, S.J., Reimer, A., Verghese, B., Lok, M., Ziegler, J., Farber, J., Pagotto, F., Graham, M., Nadon, C.A. \& Gilmour, M.W. (2012). Sequence typing confirms that a predominant Listeria monocytogenes clone caused human listeriosis cases and outbreaks in Canada from 1988 to 2010. Journal of Clinical Microbiology, 50, 1748-1751. 
Knowles, M., Lambert, D., Huszczynski, G., Gauthier, M. \& Blais, B.W. (2015a). PCR for the specific selection of an Escherichia coli O157:H7 laboratory control strain. Journal of Food Protection, 78, 1738-1744.

Knowles, M., Lambert, D., Huszczynski, G., Gauthier, M. \& Blais, B.W. (2015b). PCR for the Specific Detection of an Escherichia coli O157:H7 Laboratory Control Strain. Journal of Food Protection, 78, 1738-1744.

Knowles, M., Stinson, S., Lambert, D., Carrillo, C., Koziol, A., Gauthier, M. \& Blais, B. (2016). Genomic tools for customized recovery and detection of foodborne Shiga toxigenic Escherichia coli. Journal of Food Protection, 79, 2066-2077.

Knudsen, P.K., Gammelsrud, K.W., Alfsnes, K., Steinbakk, M., Abrahamsen, T.G., Müller, F. \& Bohlin, J. (2018). Transfer of a bla CTX-M-1 -carrying plasmid between different Escherichia coli strains within the human gut explored by whole genome sequencing analyses. Scientific Reports, 8, 280.

Koskiniemi, S., Pränting, M., Gullberg, E., Näsvall, J. \& Andersson, D.I. (2011). Activation of cryptic aminoglycoside resistance in Salmonella enterica. Molecular Microbiology, 80, 1464-1478.

Kothari, A., Wu, Y.-W., Charrier, M., Rajeev, L., Rocha, A.M., Paradis, C.J., Hazen, T.C., Singer, S.W. \& Mukhopadhyay, A. (2017). Plasmid DNA analysis of pristine groundwater microbial communities reveal extensive presence of metal resistance genes. bioRxiv, 113860.

Kottara, A., Hall, J.P.J. \& Brockhurst, M.A. (2020). The proficiency of the original host species determines community-level plasmid dynamics. bioRxiv, 2020.10.13.337451.

Kovacevic, J., Ziegler, J., Wałecka-Zacharska, E., Reimer, A., Kitts, D.D. \& Gilmour, M.W. (2016). Tolerance of Listeria monocytogenes to quaternary ammonium sanitizers is mediated by a novel efflux pump encoded by emrE. Appl. Environ. Microbiol., 82, 939-953.

Krahn, T., Wibberg, D., Maus, I., Winkler, A., Bontron, S., Sczyrba, A., Nordmann, P., Pühler, A., Poirel, L. \& Schlüter, A. (2016). Intraspecies Transfer of the Chromosomal Acinetobacter baumannii blaNDM-1 Carbapenemase Gene. Antimicrobial Agents and Chemotherapy, 60, 3032-3040.

Kram, K.E., Geiger, C., Ismail, W.M., Lee, H., Tang, H., Foster, P.L. \& Finkel, S.E. (2017). Adaptation of Escherichia coli to Long-Term Serial Passage in Complex Medium: Evidence of Parallel Evolution. mSystems, 2.

Kryachko, Y., Semler, D., Vogrinetz, J., Lemke, M., Irvine, R., Davidson, J., Links, M.G., McCarthy, E.L., Haug, B. \& Hemmingsen, S.M. (2017). Analyses of 16S rRNA and cpn60 gene sequences provide complementary information about 
potentially useful and harmful oil field microbiota. International Biodeterioration \& Biodegradation, 123, 320-327.

Kuang, D., Zhang, J., Xu, X., Shi, W., Yang, X., Su, X., Shi, X. \& Meng, J. (2018). Increase in Ceftriaxone Resistance and Widespread Extended-Spectrum $\beta$ Lactamases Genes Among Salmonella enterica from Human and Nonhuman Sources. Foodborne Pathogens and Disease, 15, 770-775.

Kücken, D., Feucht, H.-H. \& Kaulfers, P.-M. (2000). Association of qacE and qacE $\Delta 1$ with multiple resistance to antibiotics and antiseptics in clinical isolates of Gramnegative bacteria. FEMS Microbiology Letters, 183, 95-98.

Kunin, V., Copeland, A., Lapidus, A., Mavromatis, K. \& Hugenholtz, P. (2008). A Bioinformatician's Guide to Metagenomics. Microbiology and Molecular Biology Reviews : $M M B R, \mathbf{7 2}, 557-578$.

Labadene, H., Messai, Y., Ammari, H., Alouache, S., Verdet, C., Bakour, R. \& Arlet, G. (2009). Prevalence of plasmid-mediated AmpC beta-lactamases among Enterobacteriaceae in Algiers hospitals. International Journal of Antimicrobial Agents, 34, 340-342.

Lagacé-Wiens, P.R.S., Adam, H.J., Poutanen, S., Baxter, M.R., Denisuik, A.J., Golden, A.R., Nichol, K.A., Walkty, A., Karlowsky, J.A., Mulvey, M.R., Golding, G., Hoban, D.J., Zhanel, G.G., Canward, C.A.R.A. (CARA) and, Zhanel, G.G., Hoban, D.J., Adam, H.J., Baxter, M.R., Nichol, K.A., Lagacé-Wiens, P.R.S., Walkty, A., Karlowsky, J.A., Blondeau, J., Slinger, R., Davidson, R., Zhanel, G., Hoban, D., Delport, J., Ellis, C., Laverdière, M., Loo, V., Poutanen, S., Fuller, J., Roscoe, D., Desjardins, M., Matukas, L., Goyette, M., Lee, C., Carignan, A., Bergevin, M. \& Pelletier, R. (2019). Trends in antimicrobial resistance over 10 years among key bacterial pathogens from Canadian hospitals: results of the CANWARD study 2007-16. Journal of Antimicrobial Chemotherapy, 74, iv22iv31.

Lai, C.-C., Wang, C.-Y., Chu, C.-C., Tan, C.-K., Lu, C.-L., Lee, Y.-C., Huang, Y.-T., Lee, P.-I. \& Hsueh, P.-R. (2011). Correlation between antibiotic consumption and resistance of Gram-negative bacteria causing healthcare-associated infections at a university hospital in Taiwan from 2000 to 2009. The Journal of Antimicrobial Chemotherapy, 66, 1374-1382.

Lambert, D., Carrillo, C.D., Koziol, A.G., Manninger, P. \& Blais, B.W. (2015). GeneSippr: A rapid whole-genome approach for the identification and characterization of foodborne pathogens such as priority Shiga toxigenic Escherichia coli. PloS One, 10, e0122928.

Lambert, D., Pightling, A., Griffiths, E., Van Domselaar, G., Evans, P., Berthelet, S., Craig, D., Chandry, P.S., Stones, R., Brinkman, F., Angers-Loustau, A., Kreysa, J., Tong, W. \& Blais, B. (2017). Baseline practices for the application of genomic 
data supporting regulatory food safety. Journal of AOAC International, 100, 721731.

Landers, T.F., Cohen, B., Wittum, T.E. \& Larson, E.L. (2012). A Review of Antibiotic Use in Food Animals: Perspective, Policy, and Potential. Public Health Reports, 127, 4-22.

Lanza, V.F., Baquero, F., Martinez, J.L., Ramos-Ruiz, R., Gonzalez-Zorn, B., Andremont, A., Sanchez-Valenzuela, A., Ehrlich, D., Kennedy, S., Ruppe, E., Schaik, W. van, Willems, R.J., Cruz, F. de la \& Coque, T.M. (2017). In-Depth Resistome Analysis by Targeted Metagenomics. bioRxiv, 104224.

Larsen, M.V., Cosentino, S., Rasmussen, S., Friis, C., Hasman, H., Marvig, R.L., Jelsbak, L., Sicheritz-Pontén, T., Ussery, D.W., Aarestrup, F.M. \& Lund, O. (2012). Multilocus sequence typing of total-genome-sequenced bacteria. Journal of Clinical Microbiology, 50, 1355-1361.

Laxminarayan, R., Duse, A., Wattal, C., Zaidi, A.K.M., Wertheim, H.F.L., Sumpradit, N., Vlieghe, E., Hara, G.L., Gould, I.M., Goossens, H., Greko, C., So, A.D., Bigdeli, M., Tomson, G., Woodhouse, W., Ombaka, E., Peralta, A.Q., Qamar, F.N., Mir, F., Kariuki, S., Bhutta, Z.A., Coates, A., Bergstrom, R., Wright, G.D., Brown, E.D. \& Cars, O. (2013). Antibiotic resistance - the need for global solutions. The Lancet Infectious Diseases, 13, 1057-1098.

Lázár, V., Singh, G.P., Spohn, R., Nagy, I., Horváth, B., Hrtyan, M., Busa-Fekete, R., Bogos, B., Méhi, O., Csörgő, B., Pósfai, G., Fekete, G., Szappanos, B., Kégl, B., Papp, B. \& Pál, C. (2013). Bacterial evolution of antibiotic hypersensitivity. Molecular Systems Biology, 9, 700.

Lee, J.S., Mackie, R.S., Harrison, T., Shariat, B., Kind, T., Kehl, T., Löchelt, M., Boucher, C. \& VandeWoude, S. (2017). Targeted Enrichment for Pathogen Detection and Characterization in Three Felid Species. Journal of Clinical Microbiology, 55, 1658-1670.

Lei, C.-W., Kong, L.-H., Ma, S.-Z., Liu, B.-H., Chen, Y.-P., Zhang, A.-Y. \& Wang, H.N. (2017). A novel type $1 / 2$ hybrid IncC plasmid carrying fifteen antimicrobial resistance genes recovered from Proteus mirabilis in China. Plasmid, 93, 1-5.

Leong, D., Alvarez-Ordóñez, A. \& Jordan, K. (2014). Monitoring occurrence and persistence of Listeria monocytogenes in foods and food processing environments in the Republic of Ireland. Frontiers in Microbiology, 5, 436.

Levy, S. (2014). Reduced Antibiotic Use in Livestock: How Denmark Tackled Resistance. Environmental Health Perspectives, 122, A160-A165.

Li, B., Yang, Y., Ma, L., Ju, F., Guo, F., Tiedje, J.M. \& Zhang, T. (2015). Metagenomic and network analysis reveal wide distribution and co-occurrence of environmental antibiotic resistance genes. The ISME Journal, 9, 2490-2502. 
Li, W. \& Godzik, A. (2006). Cd-hit: a fast program for clustering and comparing large sets of protein or nucleotide sequences. Bioinformatics (Oxford, England), 22, $1658-1659$.

Li, W., Li, Y., Wu, Y., Cui, Y., Liu, Y., Shi, X., Zhang, Q., Chen, Q., Sun, Q. \& Hu, Q. (2016). Phenotypic and genetic changes in the life cycle of small colony variants of Salmonella enterica serotype Typhimurium induced by streptomycin. Annals of Clinical Microbiology and Antimicrobials, 15, 37.

Licht, T.R., Christensen, B.B., Krogfelt, K.A. \& Molin, S. (1999). Plasmid transfer in the animal intestine and other dynamic bacterial populations: the role of community structure and environment. Microbiology, 145, 2615-2622.

Licker, M., Anghel, A., Moldovan, R., Hogea, E., Muntean, D., Horhat, F., Seclaman, E., Tamas, L., Anghel, M. \& Baditoiu, L. (2015). Genotype-phenotype correlation in multiresistant Escherichia coli and Klebsiella pneumoniae strains isolated in Western Romania. European Review for Medical and Pharmacological Sciences, 19, 1888-1894.

Lin, J., Zhou, D., Steitz, T.A., Polikanov, Y.S. \& Gagnon, M.G. (2018). RibosomeTargeting Antibiotics: Modes of Action, Mechanisms of Resistance, and Implications for Drug Design. Annual Review of Biochemistry, 87, 451-478.

Links, M.G., Dumonceaux, T.J., Hemmingsen, S.M. \& Hill, J.E. (2012). The Chaperonin-60 Universal Target Is a Barcode for Bacteria That Enables De Novo Assembly of Metagenomic Sequence Data. PLoS ONE, 7.

Liu, C.M., Aziz, M., Kachur, S., Hsueh, P.-R., Huang, Y.-T., Keim, P. \& Price, L.B. (2012). BactQuant: An enhanced broad-coverage bacterial quantitative real-time PCR assay. BMC Microbiology, 12, 56.

Liu, L., Kreft, J. \& Vigolo, D. (2019). The effect of antibiotic and nutrient limitation to antibiotic resistant bacteria in single-cell level. Access Microbiology, 1, 203.

Liu, Z., Deng, D., Lu, H., Sun, J., Lv, L., Li, S., Peng, G., Ma, X., Li, J., Li, Z., Rong, T. \& Wang, G. (2020). Evaluation of Machine Learning Models for Predicting Antimicrobial Resistance of Actinobacillus pleuropneumoniae From Whole Genome Sequences. Frontiers in Microbiology, 11.

Livermore, D.M. (2001). Of Pseudomonas, porins, pumps and carbapenems. Journal of Antimicrobial Chemotherapy, 47, 247-250.

Livermore, D.M. (2002). Multiple Mechanisms of Antimicrobial Resistance in Pseudomonas aeruginosa: Our Worst Nightmare? Clinical Infectious Diseases, 34, 634-640. 
Llor, C. \& Bjerrum, L. (2014). Antimicrobial resistance: risk associated with antibiotic overuse and initiatives to reduce the problem. Therapeutic Advances in Drug Safety, 5, 229-241.

Lloyd-Price, J., Mahurkar, A., Rahnavard, G., Crabtree, J., Orvis, J., Hall, A.B., Brady, A., Creasy, H.H., McCracken, C., Giglio, M.G., McDonald, D., Franzosa, E.A., Knight, R., White, O. \& Huttenhower, C. (2017). Strains, functions and dynamics in the expanded Human Microbiome Project. Nature, 550, 61-66.

Lob, S.H., Nicolle, L.E., Hoban, D.J., Kazmierczak, K.M., Badal, R.E. \& Sahm, D.F. (2016). Susceptibility patterns and ESBL rates of Escherichia coli from urinary tract infections in Canada and the United States, SMART 2010-2014. Diagnostic Microbiology and Infectious Disease, 85, 459-465.

Loncaric, I., Stalder, G.L., Mehinagic, K., Rosengarten, R., Hoelzl, F., Knauer, F. \& Walzer, C. (2013). Comparison of ESBL - And AmpC Producing Enterobacteriaceae and Methicillin-Resistant Staphylococcus aureus (MRSA) Isolated from Migratory and Resident Population of Rooks (Corvus frugilegus) in Austria. PLoS ONE, 8.

Lopatkin, A.J., Meredith, H.R., Srimani, J.K., Pfeiffer, C., Durrett, R. \& You, L. (2017). Persistence and reversal of plasmid-mediated antibiotic resistance. Nature Communications, 8, 1689.

Low, A.J., Koziol, A.G., Manninger, P.A., Blais, B. \& Carrillo, C.D. (2019). ConFindr: rapid detection of intraspecies and cross-species contamination in bacterial wholegenome sequence data. PeerJ, 7, e6995.

Lundén, J.M., Autio, T.J. \& Korkeala, H.J. (2002). Transfer of persistent Listeria monocytogenes contamination between food-processing plants associated with a dicing machine. Journal of Food Protection, 65, 1129-1133.

Luo, C., Tsementzi, D., Kyrpides, N.C. \& Konstantinidis, K.T. (2012). Individual genome assembly from complex community short-read metagenomic datasets. The ISME Journal, 6, 898-901.

Macesic, N., Polubriaginof, F. \& Tatonetti, N.P. (2017). Machine learning: novel bioinformatics approaches for combating antimicrobial resistance. Current Opinion in Infectious Diseases, 30, 511-517.

Maertens, H., Van Coillie, E., Millet, S., Van Weyenberg, S., Sleeckx, N., Meyer, E., Zoons, J., Dewulf, J. \& De Reu, K. (2020). Repeated disinfectant use in broiler houses and pig nursery units does not affect disinfectant and antibiotic susceptibility in Escherichia coli field isolates. BMC Veterinary Research, 16, 140. 
Manageiro, V., Ferreira, E., Pinto, M., Fonseca, F., Ferreira, M., Bonnet, R. \& Caniça, M. (2015). Two novel CMY-2-type $\beta$-lactamases encountered in clinical Escherichia coli isolates. Annals of Clinical Microbiology and Antimicrobials, 14.

Manyi-Loh, C., Mamphweli, S., Meyer, E. \& Okoh, A. (2018). Antibiotic Use in Agriculture and Its Consequential Resistance in Environmental Sources: Potential Public Health Implications. Molecules : A Journal of Synthetic Chemistry and Natural Product Chemistry, 23.

Márquez, M.L.F., Burgos, M.J.G., Pulido, R.P., Gálvez, A. \& López, R.L. (2018). Correlations among Resistances to Different Antimicrobial Compounds in Salmonella Strains from Hen Eggshells. Journal of Food Protection, 81, 178-185.

Martin, L.C., Weir, E.K., Poppe, C., Reid-Smith, R.J. \& Boerlin, P. (2012). Characterization of blaCMY-2 Plasmids in Salmonella and Escherichia coli Isolates from Food Animals in Canada. Applied and Environmental Microbiology, 78, 1285-1287.

Martínez-Suárez, J.V., Ortiz, S. \& López-Alonso, V. (2016). Potential impact of the resistance to quaternary ammonium disinfectants on the persistence of Listeria monocytogenes in food processing environments. Frontiers in Microbiology, 7, 638.

Mataseje, L.F., Baudry, P.J., Zhanel, G.G., Morck, D.W., Read, R.R., Louie, M. \& Mulvey, M.R. (2010). Comparison of CMY-2 plasmids isolated from human, animal, and environmental Escherichia coli and Salmonella spp. from Canada. Diagnostic Microbiology and Infectious Disease, 67, 387-391.

Maury, M.M., Tsai, Y.-H., Charlier, C., Touchon, M., Chenal-Francisque, V., Leclercq, A., Criscuolo, A., Gaultier, C., Roussel, S., Brisabois, A., Disson, O., Rocha, E.P.C., Brisse, S. \& Lecuit, M. (2016). Uncovering Listeria monocytogenes hypervirulence by harnessing its biodiversity. Nature genetics, 48, 308-313.

McArthur, A.G., Waglechner, N., Nizam, F., Yan, A., Azad, M.A., Baylay, A.J., Bhullar, K., Canova, M.J., De Pascale, G., Ejim, L., Kalan, L., King, A.M., Koteva, K., Morar, M., Mulvey, M.R., O’Brien, J.S., Pawlowski, A.C., Piddock, L.J.V., Spanogiannopoulos, P., Sutherland, A.D., Tang, I., Taylor, P.L., Thaker, M., Wang, W., Yan, M., Yu, T. \& Wright, G.D. (2013). The Comprehensive Antibiotic Resistance Database. Antimicrobial Agents and Chemotherapy, 57, 3348-3357.

McDermott, P.F., Tyson, G.H., Kabera, C., Chen, Y., Li, C., Folster, J.P., Ayers, S.L., Lam, C., Tate, H.P. \& Zhao, S. (2016). Whole-Genome Sequencing for Detecting Antimicrobial Resistance in Nontyphoidal Salmonella. Antimicrobial Agents and Chemotherapy, 60, 5515-5520.

Meier, A.B., Guldimann, C., Markkula, A., Pöntinen, A., Korkeala, H. \& Tasara, T. (2017). Comparative phenotypic and genotypic analysis of Swiss and Finnish 
Listeria monocytogenes isolates with respect to benzalkonium chloride resistance. Frontiers in Microbiology, 8, 397.

Merlin, C. (2020). Reducing the Consumption of Antibiotics: Would That Be Enough to Slow Down the Dissemination of Resistances in the Downstream Environment? Frontiers in Microbiology, 11.

Mikheil, D.M., Shippy, D.C., Eakley, N.M., Okwumabua, O.E. \& Fadl, A.A. (2012). Deletion of gene encoding methyltransferase ( $\mathrm{gidB}$ ) confers high-level antimicrobial resistance in Salmonella. The Journal of Antibiotics, 65, 185-192.

Miller, R.R., Montoya, V., Gardy, J.L., Patrick, D.M. \& Tang, P. (2013). Metagenomics for pathogen detection in public health. Genome Medicine, 5.

Minarovičová, J., Véghová, A., Mikulášová, M., Chovanová, R., Šoltýs, K., Drahovská, H. \& Kaclíková, E. (2018). Benzalkonium chloride tolerance of Listeria monocytogenes strains isolated from a meat processing facility is related to presence of plasmid-borne bcrABC cassette. Antonie van Leeuwenhoek, 111, 1913-1923.

Moffat, J., Chalmers, G., Reid-Smith, R., Mulvey, M.R., Agunos, A., Calvert, J., Cormier, A., Ricker, N., Weese, J.S. \& Boerlin, P. (2020). Resistance to extended-spectrum cephalosporins in Escherichia coli and other Enterobacterales from Canadian turkeys. PLOS ONE, 15, e0236442.

Molina, L., Udaondo, Z., Duque, E., Fernández, M., Molina-Santiago, C., Roca, A., Porcel, M., Torre, J. de la, Segura, A., Plesiat, P., Jeannot, K. \& Ramos, J.-L. (2014). Antibiotic Resistance Determinants in a Pseudomonas putida Strain Isolated from a Hospital. PLOS ONE, 9, e81604.

Monstein, H.-J., Östholm-Balkhed, Å., Nilsson, M.V., Nilsson, M., Dornbusch, K. \& Nilsson, L.E. (2007). Multiplex PCR amplification assay for the detection of blaSHV, blaTEM and blaCTX-M genes in Enterobacteriaceae. APMIS, 115, 1400-1408.

Monte, D.F., Lincopan, N., Fedorka-Cray, P.J. \& Landgraf, M. (2019). Current insights on high priority antibiotic-resistant Salmonella enterica in food and foodstuffs: a review. Current Opinion in Food Science, Food Microbiology • Functional Foods and Nutrition, 26, 35-46.

Moradigaravand, D., Martin, V., Peacock, S.J. \& Parkhill, J. (2017). Population Structure of Multidrug-Resistant Klebsiella oxytoca within Hospitals across the United Kingdom and Ireland Identifies Sharing of Virulence and Resistance Genes with K. pneumoniae. Genome Biology and Evolution, 9, 574-584.

Møretrø, T. \& Langsrud, S. (2004). Listeria monocytogenes: biofilm formation and persistence in food-processing environments. Biofilms, 1, 107-121. 
Møretrø, T., Schirmer, B.C.T., Heir, E., Fagerlund, A., Hjemli, P. \& Langsrud, S. (2017). Tolerance to quaternary ammonium compound disinfectants may enhance growth of Listeria monocytogenes in the food industry. International Journal of Food Microbiology, 241, 215-224.

Moriyama, K., Wiener-Kronish, J.P. \& Sawa, T. (2009). Protective effects of affinitypurified antibody and truncated vaccines against Pseudomonas aeruginosa Vantigen in neutropenic mice. Microbiology and Immunology, 53, 587-594.

Moura, A., Criscuolo, A., Pouseele, H., Maury, M.M., Leclercq, A., Tarr, C., Björkman, J.T., Dallman, T., Reimer, A., Enouf, V., Larsonneur, E., Carleton, H., BracqDieye, H., Katz, L.S., Jones, L., Touchon, M., Tourdjman, M., Walker, M., Stroika, S., Cantinelli, T., Chenal-Francisque, V., Kucerova, Z., Rocha, E.P.C., Nadon, C., Grant, K., Nielsen, E.M., Pot, B., Gerner-Smidt, P., Lecuit, M. \& Brisse, S. (2016). Whole genome-based population biology and epidemiological surveillance of Listeria monocytogenes. Nature Microbiology, 2, 16185.

Müller, A., Rychli, K., Zaiser, A., Wieser, C., Wagner, M. \& Schmitz-Esser, S. (2014). The Listeria monocytogenes transposon Tn6188 provides increased tolerance to various quaternary ammonium compounds and ethidium bromide. FEMS microbiology letters, 361, 166-173.

Mulvey, M.R., Soule, G., Boyd, D., Demczuk, W., Ahmed, R. \& Group, the M.S.T.C.C.S. (2003). Characterization of the First Extended-Spectrum BetaLactamase-Producing Salmonella Isolate Identified in Canada. Journal of Clinical Microbiology, 41, 460-462.

Muñoz, N., Diaz-Osorio, M., Moreno, J., Sánchez-Jiménez, M. \& Cardona-Castro, N. (2010). Development and Evaluation of a Multiplex Real-Time Polymerase Chain Reaction Procedure to Clinically Type Prevalent Salmonella enterica Serovars. The Journal of Molecular Diagnostics, 12, 220-225.

Münz, M., Mahamdallie, S., Yost, S., Rimmer, A., Poyastro-Pearson, E., Strydom, A., Seal, S., Ruark, E. \& Rahman, N. (2018). CoverView: a sequence quality evaluation tool for next generation sequencing data. Wellcome Open Research, 3.

Muurinen, J., Karkman, A. \& Virta, M. (2017). High Throughput Method for Analyzing Antibiotic Resistance Genes in Wastewater Treatment Plants. In: Antimicrobial Resistance in Wastewater Treatment Processes. Pp. 253-262. John Wiley \& Sons, Ltd.

Naditz, A.L., Dzieciol, M., Wagner, M. \& Schmitz-Esser, S. (2019). Plasmids contribute to food processing environment-associated stress survival in three Listeria monocytogenes ST121, ST8, and ST5 strains. International Journal of Food Microbiology, 299, 39-46.

Navarro, F., Perez-Trallero, E., Marimon, J.M., Aliaga, R., Gomariz, M. \& Mirelis, B. (2001). CMY-2-producing Salmonella enterica, Klebsiella pneumoniae, 
Klebsiella oxytoca, Proteus mirabilis and Escherichia coli strains isolated in Spain (October 1999-December 2000). The Journal of Antimicrobial

Chemotherapy, 48, 383-389.

Navon-Venezia, S., Kondratyeva, K. \& Carattoli, A. (2017). Klebsiella pneumoniae: a major worldwide source and shuttle for antibiotic resistance. FEMS Microbiology Reviews, 41, 252-275.

Nayfach, S. \& Pollard, K.S. (2016). Toward Accurate and Quantitative Comparative Metagenomics. Cell, 166, 1103-1116.

Nelson, M.T., Pope, C.E., Marsh, R.L., Wolter, D.J., Weiss, E.J., Hager, K.R., Vo, A.T., Brittnacher, M.J., Radey, M.C., Hayden, H.S., Eng, A., Miller, S.I., Borenstein, E. \& Hoffman, L.R. (2019). Human and Extracellular DNA Depletion for Metagenomic Analysis of Complex Clinical Infection Samples Yields Optimized Viable Microbiome Profiles. Cell Reports, 26, 2227-2240.e5.

Netikul, T. \& Kiratisin, P. (2015). Genetic Characterization of Carbapenem-Resistant Enterobacteriaceae and the Spread of Carbapenem-Resistant Klebsiella pneumoniae ST340 at a University Hospital in Thailand. PLoS ONE, 10.

Ng, C., Tay, M., Tan, B., Le, T.-H., Haller, L., Chen, H., Koh, T.H., Barkham, T.M.S., Thompson, J.R. \& Gin, K.Y.-H. (2017). Characterization of Metagenomes in Urban Aquatic Compartments Reveals High Prevalence of Clinically Relevant Antibiotic Resistance Genes in Wastewaters. Frontiers in Microbiology, 8.

Ni, J., Yan, Q. \& Yu, Y. (2013). How much metagenomic sequencing is enough to achieve a given goal? Scientific Reports, 3, 1968.

Nishimura, K., Johansen, S.K., Inaoka, T., Hosaka, T., Tokuyama, S., Tahara, Y., Okamoto, S., Kawamura, F., Douthwaite, S. \& Ochi, K. (2007). Identification of the $R s m G$ Methyltransferase Target as 16S rRNA Nucleotide G527 and Characterization of Bacillus subtilis rsmG Mutants. Journal of Bacteriology, 189, 6068-6073.

Noble, W.C., Virani, Z. \& Cree, R.G. (1992). Co-transfer of vancomycin and other resistance genes from Enterococcus faecalis NCTC 12201 to Staphylococcus aureus. FEMS microbiology letters, 72, 195-198.

Nordmann, P., Naas, T. \& Poirel, L. (2011). Global Spread of Carbapenemase-producing Enterobacteriaceae. Emerging Infectious Diseases, 17, 1791-1798.

Noyes, N.R., Weinroth, M.E., Parker, J.K., Dean, C.J., Lakin, S.M., Raymond, R.A., Rovira, P., Doster, E., Abdo, Z., Martin, J.N., Jones, K.L., Ruiz, J., Boucher, C.A., Belk, K.E. \& Morley, P.S. (2017). Enrichment allows identification of diverse, rare elements in metagenomic resistome-virulome sequencing. Microbiome, 5, 142. 
Noyes, N.R., Yang, X., Linke, L.M., Magnuson, R.J., Dettenwanger, A., Cook, S., Geornaras, I., Woerner, D.E., Gow, S.P., McAllister, T.A., Yang, H., Ruiz, J., Jones, K.L., Boucher, C.A., Morley, P.S. \& Belk, K.E. (2016). Resistome diversity in cattle and the environment decreases during beef production. eLife, $\mathbf{5}$, e13195.

Okamoto, S., Tamaru, A., Nakajima, C., Nishimura, K., Tanaka, Y., Tokuyama, S., Suzuki, Y. \& Ochi, K. (2007). Loss of a conserved 7-methylguanosine modification in 16S rRNA confers low-level streptomycin resistance in bacteria. Molecular Microbiology, 63, 1096-1106.

Okonechnikov, K., Conesa, A. \& García-Alcalde, F. (2016a). Qualimap 2: advanced multi-sample quality control for high-throughput sequencing data. Bioinformatics (Oxford, England), 32, 292-294.

Okonechnikov, K., Conesa, A. \& García-Alcalde, F. (2016b). Qualimap 2: advanced multi-sample quality control for high-throughput sequencing data. Bioinformatics, 32, 292-294.

Olaitan, A.O., Morand, S. \& Rolain, J.-M. (2014). Mechanisms of polymyxin resistance: acquired and intrinsic resistance in bacteria. Frontiers in Microbiology, $\mathbf{5}$.

Oniciuc, E.-A., Likotrafiti, E., Alvarez-Molina, A., Prieto, M., López, M. \& AlvarezOrdóñez, A. (2019). Food processing as a risk factor for antimicrobial resistance spread along the food chain. Current Opinion in Food Science, Food Toxicology • Food Safety, 30, 21-26.

Orlek, A., Stoesser, N., Anjum, M.F., Doumith, M., Ellington, M.J., Peto, T., Crook, D., Woodford, N., Walker, A.S., Phan, H. \& Sheppard, A.E. (2017). Plasmid Classification in an Era of Whole-Genome Sequencing: Application in Studies of Antibiotic Resistance Epidemiology. Frontiers in Microbiology, 8.

Ortiz, S., López-Alonso, V., Rodríguez, P. \& Martínez-Suárez, J.V. (2016). The connection between persistent, disinfectant-resistant Listeria monocytogenes strains from two geographically separate Iberian pork processing plants: evidence from comparative genome analysis. Applied and Environmental Microbiology, 82, 308-317.

Paradis, E. \& Schliep, K. (2019). ape 5.0: an environment for modern phylogenetics and evolutionary analyses in R. Bioinformatics, 35, 526-528.

Paradise, M.R., Cook, G., Poole, R.K. \& Rather, P.N. (1998). Mutations in aarE, the ubiA Homolog of Providencia stuartii, Result in High-Level Aminoglycoside Resistance and Reduced Expression of the Chromosomal Aminoglycoside 29 - N -Acetyltransferase. Antimicrobial Agents and Chemotherapy, 42, 959-962.

Pardi, F. \& Goldman, N. (2005). Species Choice for Comparative Genomics: Being Greedy Works. PLOS Genetics, 1, e71. 
Park, M.-J., Kim, T.-K., Song, W., Kim, J.-S., Kim, H.-S. \& Lee, J. (2013). An Increase in the Clinical Isolation of Acquired AmpC $\beta$-Lactamase-Producing Klebsiella pneumoniae in Korea from 2007 to 2010. Annals of Laboratory Medicine, 33, $353-355$.

Pärnänen, K.M.M., Narciso-da-Rocha, C., Kneis, D., Berendonk, T.U., Cacace, D., Do, T.T., Elpers, C., Fatta-Kassinos, D., Henriques, I., Jaeger, T., Karkman, A., Martinez, J.L., Michael, S.G., Michael-Kordatou, I., O’Sullivan, K., RodriguezMozaz, S., Schwartz, T., Sheng, H., Sørum, H., Stedtfeld, R.D., Tiedje, J.M., Giustina, S.V.D., Walsh, F., Vaz-Moreira, I., Virta, M. \& Manaia, C.M. (2019). Antibiotic resistance in European wastewater treatment plants mirrors the pattern of clinical antibiotic resistance prevalence. Science Advances, 5, eaau9124.

Paterson, D.L., Hujer, K.M., Hujer, A.M., Yeiser, B., Bonomo, M.D., Rice, L.B., Bonomo, R.A. \& Acknowledgments, the I.K.S.G. of the I.K.S.G. are listed in. (2003). Extended-Spectrum $\beta$-Lactamases in Klebsiella pneumoniae Bloodstream Isolates from Seven Countries: Dominance and Widespread Prevalence of SHVand CTX-M-Type $\beta$-Lactamases. Antimicrobial Agents and Chemotherapy, 47, 3554-3560.

Paulsen, I.T., Brown, M.H., Dunstan, S.J. \& Skurray, R.A. (1995). Molecular characterization of the staphylococcal multidrug resistance export protein QacC. Journal of Bacteriology, 177, 2827-2833.

Payne, A., Holmes, N., Clarke, T., Munro, R., Debebe, B. \& Loose, M. (2020). Nanopore adaptive sequencing for mixed samples, whole exome capture and targeted panels. bioRxiv, 2020.02.03.926956.

Pearce, H., Messager, S. \& Maillard, J.-Y. (1999). Effect of biocides commonly used in the hospital environment on the transfer of antibiotic-resistance genes in Staphylococcus aureus. Journal of Hospital Infection, 43, 101-107.

Pelt, W. van, Zee, H. van der, Wannet, W.J.B., Giessen, A.W. van de, Mevius, D.J., Bolder, N.M., Komijn, R.E. \& Duynhoven, Y.T.H.P. van. (2003). Explosive increase of Salmonella Java in poultry in the Netherlands: consequences for public health. Euro Surveillance: Bulletin Europeen Sur Les Maladies Transmissibles = European Communicable Disease Bulletin, 8, 31-35.

Pereira-Marques, J., Hout, A., Ferreira, R.M., Weber, M., Pinto-Ribeiro, I., Doorn, L.-J. van, Knetsch, C.W. \& Figueiredo, C. (2019). Impact of Host DNA and Sequencing Depth on the Taxonomic Resolution of Whole Metagenome Sequencing for Microbiome Analysis. Frontiers in Microbiology, 10.

Perez, M.F., Kurth, D., Farías, M.E., Soria, M.N., Castillo Villamizar, G.A., Poehlein, A., Daniel, R. \& Dib, J.R. (2020). First Report on the Plasmidome From a HighAltitude Lake of the Andean Puna. Frontiers in Microbiology, 11. 
Petkau, A., Mabon, P., Sieffert, C., Knox, N.C., Cabral, J., Iskander, M., Iskander, M., Weedmark, K., Zaheer, R., Katz, L.S., Nadon, C., Reimer, A., Taboada, E., Beiko, R.G., Hsiao, W., Brinkman, F., Graham, M. \& Van Domselaar, G. (2017). SNVPhyl: a single nucleotide variant phylogenomics pipeline for microbial genomic epidemiology. Microbial Genomics, 3, e000116.

Peymani, A., Naserpour-Farivar, T., Yeylagh-Beygi, M. \& Bolori, S. (2016). Emergence of CMY-2- and DHA-1-type AmpC $\beta$-lactamases in Enterobacter cloacae isolated from several hospitals of Qazvin and Tehran, Iran. Iranian Journal of Microbiology, 8, 168-174.

Philippon, A., Arlet, G. \& Jacoby, G.A. (2002). Plasmid-Determined AmpC-Type $\beta$ Lactamases. Antimicrobial Agents and Chemotherapy, 46, 1-11.

Philippon, A., Slama, P., Dény, P. \& Labia, R. (2016). A Structure-Based Classification of Class A $\beta$-Lactamases, a Broadly Diverse Family of Enzymes. Clinical Microbiology Reviews, 29, 29-57.

Phillips, I., Casewell, M., Cox, T., De Groot, B., Friis, C., Jones, R., Nightingale, C., Preston, R. \& Waddell, J. (2004). Does the use of antibiotics in food animals pose a risk to human health? A critical review of published data. Journal of Antimicrobial Chemotherapy, 53, 28-52.

Piddock, L.J., Ricci, V., McLaren, I. \& Griggs, D.J. (1998). Role of mutation in the gyrA and parC genes of nalidixic-acid-resistant Salmonella serotypes isolated from animals in the United Kingdom. Journal of Antimicrobial Chemotherapy, 41, 635-641.

Pierce, V.M., Simner, P.J., Lonsway, D.R., Roe-Carpenter, D.E., Johnson, J.K., Brasso, W.B., Bobenchik, A.M., Lockett, Z.C., Charnot-Katsikas, A., Ferraro, M.J., Thomson, R.B., Jenkins, S.G., Limbago, B.M. \& Das, S. (2017). Modified Carbapenem Inactivation Method for Phenotypic Detection of Carbapenemase Production among Enterobacteriaceae. Journal of Clinical Microbiology, 55, 2321-2333.

Pirone-Davies, C., Chen, Y., Pightling, A., Ryan, G., Wang, Y., Yao, K., Hoffmann, M. \& Allard, M.W. (2018). Genes significantly associated with lineage II food isolates of Listeria monocytogenes. BMC Genomics, 19, 708.

Plumb, I.D. (2019). Outbreak of Salmonella Newport Infections with Decreased Susceptibility to Azithromycin Linked to Beef Obtained in the United States and Soft Cheese Obtained in Mexico — United States, 2018-2019. MMWR. Morbidity and Mortality Weekly Report, 68.

Poirel, L., Dortet, L., Bernabeu, S. \& Nordmann, P. (2011). Genetic Features of blaNDM-1-Positive Enterobacteriaceae. Antimicrobial Agents and Chemotherapy, 55, 5403-5407. 
Poole, K. (2005). Efflux-mediated antimicrobial resistance. Journal of Antimicrobial Chemotherapy, 56, 20-51.

Poole, K. (2012). Bacterial stress responses as determinants of antimicrobial resistance. Journal of Antimicrobial Chemotherapy, 67, 2069-2089.

Pornsukarom, S. \& Thakur, S. (2017). Horizontal Dissemination of Antimicrobial Resistance Determinants in Multiple Salmonella Serotypes following Isolation from the Commercial Swine Operation Environment after Manure Application. Applied and Environmental Microbiology, 83, e01503-17.

Pornsukarom, S., Vliet, A.H.M. van \& Thakur, S. (2018). Whole genome sequencing analysis of multiple Salmonella serovars provides insights into phylogenetic relatedness, antimicrobial resistance, and virulence markers across humans, food animals and agriculture environmental sources. BMC Genomics, 19, 801.

Porse, A., Gumpert, H., Kubicek-Sutherland, J.Z., Karami, N., Adlerberth, I., Wold, A.E., Andersson, D.I. \& Sommer, M.O.A. (2017). Genome Dynamics of Escherichia coli during Antibiotic Treatment: Transfer, Loss, and Persistence of Genetic Elements In situ of the Infant Gut. Frontiers in Cellular and Infection Microbiology, 7, 126.

Porse, A., Schønning, K., Munck, C. \& Sommer, M.O.A. (2016). Survival and Evolution of a Large Multidrug Resistance Plasmid in New Clinical Bacterial Hosts. Molecular Biology and Evolution, 33, 2860-2873.

Qi, C., Stratton, C.W. \& Zheng, X. (2006). Phenotypic Testing of Bacterial Antimicrobial Susceptibility. In: Advanced Techniques in Diagnostic Microbiology (edited by Y.-W. Tang \& C.W. Stratton). Pp. 63-83. Boston, MA: Springer US.

Queenan, A.M. \& Bush, K. (2007). Carbapenemases: the Versatile $\beta$-Lactamases. Clinical Microbiology Reviews, 20, 440-458.

R Core Team. (2014). R: A language and environment for statistical computing. Vienna, Austria: R Foundation for Statistical Computing.

Ragon, M., Wirth, T., Hollandt, F., Lavenir, R., Lecuit, M., Le Monnier, A. \& Brisse, S. (2008). A new perspective on Listeria monocytogenes evolution. PLoS Pathogens, 4, e1000146.

Rakic-Martinez, M., Drevets, D.A., Dutta, V., Katic, V. \& Kathariou, S. (2011). Listeria monocytogenes strains selected on ciprofloxacin or the disinfectant benzalkonium chloride exhibit reduced susceptibility to ciprofloxacin, gentamicin, benzalkonium chloride, and other toxic compounds. Applied and Environmental Microbiology, 77, 8714-8721. 
Ramirez, M.S. \& Tolmasky, M.E. (2010). Aminoglycoside Modifying Enzymes. Drug resistance updates : reviews and commentaries in antimicrobial and anticancer chemotherapy, 13, 151-171.

Randall, L.P., Cooles, S.W., Osborn, M.K., Piddock, L.J.V. \& Woodward, M.J. (2004). Antibiotic resistance genes, integrons and multiple antibiotic resistance in thirtyfive serotypes of Salmonella enterica isolated from humans and animals in the UK. Journal of Antimicrobial Chemotherapy, 53, 208-216.

Reche, M.P., Ríos, J.E.G. de los, Jiménez, P.A., Rojas, A.M. \& Rotger, R. (2002). gyrA Mutations Associated with Nalidixic Acid-Resistant Salmonellae from Wild Birds. Antimicrobial Agents and Chemotherapy, 46, 3108-3109.

Rhouma, M., Romero-Barrios, P., Gaucher, M.-L. \& Bhachoo, S. (2020). Antimicrobial resistance associated with the use of antimicrobial processing aids during poultry processing operations: cause for concern? Critical Reviews in Food Science and Nutrition, 0, 1-18.

Ricker, N., Spoja, B.S., May, N. \& Chalmers, G. (2020). Incorporating the plasmidome into antibiotic resistance surveillance in animal agriculture. Plasmid, 102529.

Riley, L.W. (2020). Extraintestinal Foodborne Pathogens. Annual Review of Food Science and Technology, 11, 275-294.

Robertson, J. \& Nash, J.H.E. (2018). MOB-suite: software tools for clustering, reconstruction and typing of plasmids from draft assemblies. Microbial Genomics, 4, e000206.

Robertson, J., Yoshida, C., Kruczkiewicz, P., Nadon, C., Nichani, A., Taboada, E.N. \& Nash, J.H.E. (2018). Comprehensive assessment of the quality of Salmonella whole genome sequence data available in public sequence databases using the Salmonella in silico Typing Resource (SISTR). Microbial Genomics, 4.

Rocha, J., Fernandes, T., Riquelme, M.V., Zhu, N., Pruden, A. \& Manaia, C.M. (2019). Comparison of Culture- and Quantitative PCR-Based Indicators of Antibiotic Resistance in Wastewater, Recycled Water, and Tap Water. International Journal of Environmental Research and Public Health, 16.

Rodríguez-Baño, J., Miró, E., Villar, M., Coelho, A., Gozalo, M., Borrell, N., Bou, G., Conejo, M.C., Pomar, V., Aracil, B., Larrosa, N., Agüero, J., Oliver, A., Fernández, A., Oteo, J., Pascual, A. \& Navarro, F. (2012). Colonisation and infection due to Enterobacteriaceae producing plasmid-mediated AmpC $\beta$ lactamases. Journal of Infection, 64, 176-183.

Romanova, N., Favrin, S. \& Griffiths, M.W. (2002). Sensitivity of Listeria monocytogenes to sanitizers used in the meat processing industry. Applied and Environmental Microbiology, 68, 6405-6409. 
Romero, J.L., Grande Burgos, M.J., Pérez-Pulido, R., Gálvez, A. \& Lucas, R. (2017). Resistance to Antibiotics, Biocides, Preservatives and Metals in Bacteria Isolated from Seafoods: Co-Selection of Strains Resistant or Tolerant to Different Classes of Compounds. Frontiers in Microbiology, 8.

Rosengren, L.B., Waldner, C.L. \& Reid-Smith, R.J. (2009). Associations between Antimicrobial Resistance Phenotypes, Antimicrobial Resistance Genes, and Virulence Genes of Fecal Escherichia coli Isolates from Healthy Grow-Finish Pigs. Applied and Environmental Microbiology, 75, 1373-1380.

Rossolini, G.M., Zanchi, A., Chiesurin, A., Amicosante, G., Satta, G. \& Guglielmetti, P. (1995). Distribution of cphA or related carbapenemase-encoding genes and production of carbapenemase activity in members of the genus Aeromonas. Antimicrobial Agents and Chemotherapy, 39, 346-349.

Routh, J.A., Pringle, J., Mohr, M., Bidol, S., Arends, K., Adams-Cameron, M., Hancock, W.T., Kissler, B., Rickert, R., Folster, J., Tolar, B., Bosch, S., Behravesh, C.B., Williams, I.T. \& Gieraltowski, L. (2015). Nationwide outbreak of multidrugresistant Salmonella Heidelberg infections associated with ground turkey: United States, 2011. Epidemiology \& Infection, 143, 3227-3234.

Rozwandowicz, M., Brouwer, M.S.M., Fischer, J., Wagenaar, J.A., Gonzalez-Zorn, B., Guerra, B., Mevius, D.J. \& Hordijk, J. (2018). Plasmids carrying antimicrobial resistance genes in Enterobacteriaceae. Journal of Antimicrobial Chemotherapy, 73, 1121-1137.

Ruiz, J. (2003). Mechanisms of resistance to quinolones: target alterations, decreased accumulation and DNA gyrase protection. Journal of Antimicrobial Chemotherapy, 51, 1109-1117.

Ruiz, J., Castro, D., Goñi, P., Santamaria, J.A., Borrego, J.J. \& Vila, J. (1997). Analysis of the mechanism of quinolone resistance in nalidixic acid-resistant clinical isolates of Salmonella serotype Typhimurium. Journal of Medical Microbiology, 46, 623-628.

Saavedra, M.J., Peixe, L., Sousa, J.C., Henriques, I., Alves, A. \& Correia, A. (2003). SfhI, a Subclass B2 Metallo- $\beta$-Lactamase from a Serratia fonticola Environmental Isolate. Antimicrobial Agents and Chemotherapy, 47, 2330-2333.

Saliu, E.-M., Zentek, J. \& Vahjen, W. (2020). In vitro conjugation kinetics of AmpC, broad spectrum and extended-spectrum beta-lactamase-producing Escherichia coli donors and various Enterobacteriaceae recipients. BMC microbiology, 20, 133.

Sarowska, J., Drulis-Kawa, Z., Guz, K., Jankowski, S. \& Wojinicz, D. (2009). Conjugative Transfer of Plasmid Encoding Extended-Spectrum Beta-Lactamase to Recipient Salmonella Strains. Advances in Clinical and Experimental Medicine, 18, 63-70. 
Schaenzer, A.J. \& Wright, G.D. (2020). Antibiotic Resistance by Enzymatic Modification of Antibiotic Targets. Trends in Molecular Medicine, 26, 768-782.

Schmieder, R. \& Edwards, R. (2011). Insights into antibiotic resistance through metagenomic approaches. Future Microbiology, 7, 73-89.

Schneider, I. \& Bauernfeind, A. (2015). Intrinsic Carbapenem-Hydrolyzing Oxacillinases from Members of the Genus Pandoraea. Antimicrobial Agents and Chemotherapy, 59, 7136-7141.

Schneider, J.L., White, P.L., Weiss, J., Norton, D., Lidgard, J., Gould, L.H., Yee, B., Vugia, D.J. \& Mohle-Boetani, J. (2011). Multistate Outbreak of MultidrugResistant Salmonella Newport Infections Associated with Ground Beef, October to December 2007. Journal of Food Protection, 74, 1315-1319.

Schwartz, T., Kohnen, W., Jansen, B. \& Obst, U. (2003). Detection of antibiotic-resistant bacteria and their resistance genes in wastewater, surface water, and drinking water biofilms. FEMS Microbiology Ecology, 43, 325-335.

Scornec, H., Bellanger, X., Guilloteau, H., Groshenry, G. \& Merlin, C. (2017). Inducibility of Tn916 conjugative transfer in Enterococcus faecalis by subinhibitory concentrations of ribosome-targeting antibiotics. The Journal of Antimicrobial Chemotherapy, 72, 2722-2728.

Seemann, T. (2014). Prokka: rapid prokaryotic genome annotation. Bioinformatics (Oxford, England), 30, 2068-2069.

Segatore, B., Massidda, O., Satta, G., Setacci, D. \& Amicosante, G. (1993). High specificity of $c p h A$-encoded metallo-beta-lactamase from Aeromonas hydrophila AE036 for carbapenems and its contribution to beta-lactam resistance. Antimicrobial Agents and Chemotherapy, 37, 1324-1328.

Seier-Petersen, M.A., Jasni, A., Aarestrup, F.M., Vigre, H., Mullany, P., Roberts, A.P. \& Agersø, Y. (2014). Effect of subinhibitory concentrations of four commonly used biocides on the conjugative transfer of Tn916 in Bacillus subtilis. Journal of Antimicrobial Chemotherapy, 69, 343-348.

Seiffert, S.N., Hilty, M., Perreten, V. \& Endimiani, A. (2013). Extended-spectrum cephalosporin-resistant gram-negative organisms in livestock: An emerging problem for human health? Drug Resistance Updates, 16, 22-45.

Seltmann, G. \& Holst, O. (2013). The Bacterial Cell Wall. Springer Science \& Business Media.

Shaikh, S., Fatima, J., Shakil, S., Rizvi, S.Mohd.D. \& Kamal, M.A. (2015). Antibiotic resistance and extended spectrum beta-lactamases: Types, epidemiology and treatment. Saudi Journal of Biological Sciences, 22, 90-101. 
Sheng, W.-H., Badal, R.E., Hsueh, P.-R., \& SMART Program. (2013). Distribution of extended-spectrum $\beta$-lactamases, AmpC $\beta$-lactamases, and carbapenemases among Enterobacteriaceae isolates causing intra-abdominal infections in the Asia-Pacific region: results of the study for Monitoring Antimicrobial Resistance Trends (SMART). Antimicrobial Agents and Chemotherapy, 57, 2981-2988.

Shipp, C.R. \& Rowe, B. (1980). A mechanised microtechnique for Salmonella serotyping. Journal of Clinical Pathology, 33, 595-597.

Sidjabat, H.E., Seah, K.Y., Coleman, L., Sartor, A., Derrington, P., Heney, C., Faoagali, J., Nimmo, G.R. \& Paterson, D.L. (2014). Expansive spread of IncI1 plasmids carrying blaCMY-2 amongst Escherichia coli. International Journal of Antimicrobial Agents, 44, 203-208.

Sievert, D.M., Ricks, P., Edwards, J.R., Schneider, A., Patel, J., Srinivasan, A., Kallen, A., Limbago, B., Fridkin, S. \& Facilities, for the N.H.S.N. (NHSN) T. and P.N. (2013). Antimicrobial-Resistant Pathogens Associated with HealthcareAssociated Infections: Summary of Data Reported to the National Healthcare Safety Network at the Centers for Disease Control and Prevention, 2009-2010. Infection Control and Hospital Epidemiology, 34, 1-14.

Sims, D., Sudbery, I., Ilott, N.E., Heger, A. \& Ponting, C.P. (2014). Sequencing depth and coverage: key considerations in genomic analyses. Nature Reviews Genetics, 15, 121-132.

Sims, N. \& Kasprzyk-Hordern, B. (2020). Future perspectives of wastewater-based epidemiology: Monitoring infectious disease spread and resistance to the community level. Environment International, 139, 105689.

Singer, A.C., Shaw, H., Rhodes, V. \& Hart, A. (2016). Review of Antimicrobial Resistance in the Environment and Its Relevance to Environmental Regulators. Frontiers in Microbiology, 7.

Singtohin, S., Chanawong, A., Lulitanond, A., Sribenjalux, P., Auncharoen, A., Kaewkes, W., Songsri, J. \& Pienthaweechai, K. (2010). CMY-2, CMY-8b, and DHA-1 plasmid-mediated AmpC $\beta$-lactamases among clinical isolates of Escherichia coli and Klebsiella pneumoniae from a university hospital, Thailand. Diagnostic Microbiology and Infectious Disease, 68, 271-277.

Sirous, M., Khosravi, A.D., Tabandeh, M.R., Salmanzadeh, S., Ahmadkhosravi, N. \& Amini, S. (2018). Molecular detection of rifampin, isoniazid, and ofloxacin resistance in Iranian isolates of Mycobacterium tuberculosis by high-resolution melting analysis. Infection and Drug Resistance, 11, 1819-1829.

Sköld, O. (2000). Sulfonamide resistance: mechanisms and trends. Drug Resistance Updates: Reviews and Commentaries in Antimicrobial and Anticancer Chemotherapy, 3, 155-160. 
Smalla, K., Heuer, H., Götz, A., Niemeyer, D., Krögerrecklenfort, E. \& Tietze, E. (2000). Exogenous Isolation of Antibiotic Resistance Plasmids from Piggery Manure Slurries Reveals a High Prevalence and Diversity of IncQ-Like Plasmids. Applied and Environmental Microbiology, 66, 4854-4862.

Soballe, B. \& Poole, R.K. (1999). Microbial ubiquinones: multiple roles in respiration, gene regulation and oxidative stress management. Microbiology, 145, 1817-1830.

Sogin, M.L., Morrison, H.G., Huber, J.A., Welch, D.M., Huse, S.M., Neal, P.R., Arrieta, J.M. \& Herndl, G.J. (2006). Microbial diversity in the deep sea and the underexplored "rare biosphere." Proceedings of the National Academy of Sciences, 103, 12115-12120.

Somboro, A.M., Sekyere, J.O., Amoako, D.G., Essack, S.Y. \& Bester, L.A. (2018). Diversity and Proliferation of Metallo- $\beta$-Lactamases: a Clarion Call for Clinically Effective Metallo- $\beta$-Lactamase Inhibitors. Applied and Environmental Microbiology, 84.

Souvorov, A., Agarwala, R. \& Lipman, D.J. (2018). SKESA: strategic k-mer extension for scrupulous assemblies. Genome Biology, 19, 153.

Speer, B.S., Shoemaker, N.B. \& Salyers, A.A. (1992). Bacterial resistance to tetracycline: mechanisms, transfer, and clinical significance. Clinical Microbiology Reviews, 5, 387-399.

Springer, B., Kidan, Y.G., Prammananan, T., Ellrott, K., Böttger, E.C. \& Sander, P. (2001). Mechanisms of streptomycin resistance: selection of mutations in the $16 \mathrm{~S}$ rRNA gene conferring resistance. Antimicrobial Agents and Chemotherapy, 45, $2877-2884$.

Stanton, I.C., Bethel, A., Leonard, A.F.C., Gaze, W.H. \& Garside, R. (2020). What is the research evidence for antibiotic resistance exposure and transmission to humans from the environment? A systematic map protocol. Environmental Evidence, $\mathbf{9}$, 12.

Stecher, B., Denzler, R., Maier, L., Bernet, F., Sanders, M.J., Pickard, D.J., Barthel, M., Westendorf, A.M., Krogfelt, K.A., Walker, A.W., Ackermann, M., Dobrindt, U., Thomson, N.R. \& Hardt, W.-D. (2012). Gut inflammation can boost horizontal gene transfer between pathogenic and commensal Enterobacteriaceae. Proceedings of the National Academy of Sciences, 109, 1269-1274.

Steel, M. (2005). Phylogenetic Diversity and the Greedy Algorithm. Systematic Biology, $\mathbf{5 4}, 527-529$.

Steen, A.D., Crits-Christoph, A., Carini, P., DeAngelis, K.M., Fierer, N., Lloyd, K.G. \& Cameron Thrash, J. (2019). High proportions of bacteria and archaea across most biomes remain uncultured. The ISME Journal, 13, 3126-3130. 
Stiefel, P., Mauerhofer, S., Schneider, J., Maniura-Weber, K., Rosenberg, U. \& Ren, Q. (2016). Enzymes Enhance Biofilm Removal Efficiency of Cleaners. Antimicrobial Agents and Chemotherapy, 60, 3647-3652.

Su, L.-H., Chen, H.-L., Chia, J.-H., Liu, S.-Y., Chu, C., Wu, T.-L. \& Chiu, C.-H. (2006). Distribution of a transposon-like element carrying blaCMY-2 among Salmonella and other Enterobacteriaceae. Journal of Antimicrobial Chemotherapy, 57, 424429.

Su, L.-H., Chiu, C.-H., Chu, C. \& Ou, J.T. (2004). Antimicrobial Resistance in Nontyphoid Salmonella Serotypes: A Global Challenge. Clinical Infectious Diseases, 39, 546-551.

Suttner, B., Johnston, E.R., Orellana, L.H., Rodriguez-R, L.M., Hatt, J.K., Carychao, D., Carter, M.Q., Cooley, M.B. \& Konstantinidis, K.T. (2020). Metagenomics as a Public Health Risk Assessment Tool in a Study of Natural Creek Sediments Influenced by Agricultural and Livestock Runoff: Potential and Limitations. Applied and Environmental Microbiology, 86.

Suzuki, H., Yano, H., Brown, C.J. \& Top, E.M. (2010). Predicting Plasmid Promiscuity Based on Genomic Signature. Journal of Bacteriology, 192, 6045-6055.

Suzuki, M., Suzuki, S., Matsui, M., Hiraki, Y., Kawano, F. \& Shibayama, K. (2014). A subclass B3 metallo- $\beta$-lactamase found in Pseudomonas alcaligenes. Journal of Antimicrobial Chemotherapy, 69, 1430-1432.

Tacão, M., Correia, A. \& Henriques, I.S. (2015). Low Prevalence of CarbapenemResistant Bacteria in River Water: Resistance Is Mostly Related to Intrinsic Mechanisms. Microbial Drug Resistance, 21, 497-506.

Tamma, P.D., Opene, B.N.A., Gluck, A., Chambers, K.K., Carroll, K.C. \& Simner, P.J. (2017). Comparison of 11 Phenotypic Assays for Accurate Detection of Carbapenemase-Producing Enterobacteriaceae. Journal of Clinical Microbiology, 55, 1046-1055.

Tang, K.L., Caffrey, N.P., Nóbrega, D.B., Cork, S.C., Ronksley, P.E., Barkema, H.W., Polachek, A.J., Ganshorn, H., Sharma, N., Kellner, J.D. \& Ghali, W.A. (2017). Restricting the use of antibiotics in food-producing animals and its associations with antibiotic resistance in food-producing animals and human beings: a systematic review and meta-analysis. The Lancet. Planetary Health, 1, e316e327.

Testerman, T.L., Conn, P.B., Mobley, H.L.T. \& McGee, D.J. (2006). Nutritional Requirements and Antibiotic Resistance Patterns of Helicobacter Species in Chemically Defined Media. Journal of Clinical Microbiology, 44, 1650-1658.

Thaller, M.C., Borgianni, L., Di Lallo, G., Chong, Y., Lee, K., Dajcs, J., Stroman, D. \& Rossolini, G.M. (2011). Metallo- $\beta$-Lactamase Production by Pseudomonas 
otitidis: a Species-Related Trait. Antimicrobial Agents and Chemotherapy, 55, $118-123$.

Thomas, M., Webb, M., Ghimire, S., Blair, A., Olson, K., Fenske, G.J., Fonder, A.T., Christopher-Hennings, J., Brake, D. \& Scaria, J. (2017). Metagenomic characterization of the effect of feed additives on the gut microbiome and antibiotic resistome of feedlot cattle. Scientific Reports, 7, 12257.

Thomson, K.S., Sanders, C.C. \& Washington, J.A. (1993). Ceftazidime resistance in Hafnia alvei. Antimicrobial Agents and Chemotherapy, 37, 1375-1376.

Threlfall, J., Levent, B., Hopkins, K.L., Pinna, E. de, Ward, L.R. \& Brown, D.J. (2005). Multidrug-resistant Salmonella Java. Emerging Infectious Diseases, 11, 170-171.

Tran, T., Checkley, S., Caffrey, N., Cassis, R., Mainali, C., Gow, S., Agunos, A. \& Liljebjelke, K. (2020). Genetic characterization of AmpC and extended-spectrum beta-lactamase (ESBL) phenotypes in Escherichia coli and Salmonella from Alberta poultry. bioRxiv, 2020.08.11.246645.

Treangen, T.J., Ondov, B.D., Koren, S. \& Phillippy, A.M. (2014). The Harvest suite for rapid core-genome alignment and visualization of thousands of intraspecific microbial genomes. Genome Biology, 15, 524.

Trudel, M.V., Vincent, A.T., Attéré, S.A., Labbé, M., Derome, N., Culley, A.I. \& Charette, S.J. (2016). Diversity of antibiotic-resistance genes in Canadian isolates of Aeromonas salmonicida subsp. salmonicida: dominance of pSN254b and discovery of pAsa8. Scientific Reports, 6, 35617.

Turner, P.E., Cooper, V.S. \& Lenski, R.E. (1998). Tradeoff Between Horizontal and Vertical Modes of Transmission in Bacterial Plasmids. Evolution, 52, 315-329.

Tyson, G.H., Li, C., Ayers, S., McDermott, P.F. \& Zhao, S. (2016). Using whole-genome sequencing to determine appropriate streptomycin epidemiological cutoffs for Salmonella and Escherichia coli. FEMS microbiology letters, 363, fnw009.

Tyson, G.H., McDermott, P.F., Li, C., Chen, Y., Tadesse, D.A., Mukherjee, S., BodeisJones, S., Kabera, C., Gaines, S.A., Loneragan, G.H., Edrington, T.S., Torrence, M., Harhay, D.M. \& Zhao, S. (2015). WGS accurately predicts antimicrobial resistance in Escherichia coli. Journal of Antimicrobial Chemotherapy, 70, $2763-$ 2769.

Tzouvelekis, L.S., Tzelepi, E., Tassios, P.T. \& Legakis, N.J. (2000). CTX-M-type $\beta$ lactamases: an emerging group of extended-spectrum enzymes. International Journal of Antimicrobial Agents, 14, 137-142.

Udikovic-Kolic, N., Wichmann, F., Broderick, N.A. \& Handelsman, J. (2014). Bloom of resident antibiotic-resistant bacteria in soil following manure fertilization. Proceedings of the National Academy of Sciences, 111, 15202-15207. 
Uelze, L., Borowiak, M., Deneke, C., Szabó, I., Fischer, J., Tausch, S.H. \& Malorny, B. (2020). Performance and Accuracy of Four Open-Source Tools for In Silico Serotyping of Salmonella spp. Based on Whole-Genome Short-Read Sequencing Data. Applied and Environmental Microbiology, 86.

Uzunović, S., Ibrahimagić, A. \& Bedenić, B. (2016). Molecular epidemiology and antimicrobial susceptibility of AmpC- and/or extended-spectrum (ESBL) $\beta$ lactamase- producing Proteus spp. clinical isolates in Zenica-Doboj Canton, Bosnia and Herzegovina. International Journal of Infectious Diseases, 53, 43.

Van Camp, P.-J., Haslam, D.B. \& Porollo, A. (2020). Prediction of Antimicrobial Resistance in Gram-Negative Bacteria From Whole-Genome Sequencing Data. Frontiers in Microbiology, 11.

Van Meervenne, E., Van Coillie, E., Kerckhof, F.-M., Devlieghere, F., Herman, L., De Gelder, L.S.P., Top, E.M. \& Boon, N. (2012). Strain-Specific Transfer of Antibiotic Resistance from an Environmental Plasmid to Foodborne Pathogens. Journal of Biomedicine and Biotechnology, 2012.

Varela, A.R., Nunes, O.C. \& Manaia, C.M. (2016). Quinolone resistant Aeromonas spp. as carriers and potential tracers of acquired antibiotic resistance in hospital and municipal wastewater. Science of The Total Environment, 542, 665-671.

Verdet, C., Arlet, G., Redjeb, S.B., Hassen, A.B., Lagrange, P.H. \& Philippon, A. (1998). Characterisation of CMY-4, an AmpC-type plasmid-mediated $\beta$-lactamase in a Tunisian clinical isolate of Proteus mirabilis. FEMS Microbiology Letters, 169, $235-240$.

Vijayakumar, R. \& Sandle, T. (2019). A review on biocide reduced susceptibility due to plasmid-borne antiseptic-resistant genes-special notes on pharmaceutical environmental isolates. Journal of Applied Microbiology, 126, 1011-1022.

Vlieghe, E.R., Huang, T.-D., Phe, T., Bogaerts, P., Berhin, C., De Smet, B., Peetermans, W.E., Jacobs, J.A. \& Glupczynski, Y. (2015). Prevalence and distribution of betalactamase coding genes in third-generation cephalosporin-resistant

Enterobacteriaceae from bloodstream infections in Cambodia. European Journal of Clinical Microbiology \& Infectious Diseases: Official Publication of the European Society of Clinical Microbiology, 34, 1223-1229.

Wales, A.D. \& Davies, R.H. (2015). Co-Selection of Resistance to Antibiotics, Biocides and Heavy Metals, and Its Relevance to Foodborne Pathogens. Antibiotics, 4, 567-604.

Walker, B.J., Abeel, T., Shea, T., Priest, M., Abouelliel, A., Sakthikumar, S., Cuomo, C.A., Zeng, Q., Wortman, J., Young, S.K. \& Earl, A.M. (2014). Pilon: an integrated tool for comprehensive microbial variant detection and genome assembly improvement. PloS One, 9, e112963. 
Walther-Rasmussen, J. \& Høiby, N. (2007). Class A carbapenemases. The Journal of Antimicrobial Chemotherapy, 60, 470-482.

Wang, R., Kalchayanand, N., King, D.A., Luedtke, B.E., Bosilevac, J.M. \& Arthur, T.M. (2014). Biofilm Formation and Sanitizer Resistance of Escherichia coli O157:H7 Strains Isolated from "High Event Period" Meat Contamination. Journal of Food Protection, 77, 1982-1987.

Webber, M.A. \& Piddock, L.J.V. (2003). The importance of efflux pumps in bacterial antibiotic resistance. Journal of Antimicrobial Chemotherapy, 51, 9-11.

Webber, M.A., Whitehead, R.N., Mount, M., Loman, N.J., Pallen, M.J. \& Piddock, L.J.V. (2015). Parallel evolutionary pathways to antibiotic resistance selected by biocide exposure. Journal of Antimicrobial Chemotherapy, 70, 2241-2248.

Wegener, H.C. (2003). Antibiotics in animal feed and their role in resistance development. Current Opinion in Microbiology, 6, 439-445.

Westhoff, S., Leeuwe, T.M. van, Qachach, O., Zhang, Z., Wezel, G.P. van \& Rozen, D.E. (2017). The evolution of no-cost resistance at sub-MIC concentrations of streptomycin in Streptomyces coelicolor. The ISME journal, 11, 1168-1178.

WHO. (2017a). WHO | Antimicrobial resistance [Internet document]. WHO. URL http://www.who.int/antimicrobial-resistance/en/. Accessed 06/04/2017.

WHO. (2019). WHO | Critically important antimicrobials for human medicine 6th Revision [Internet document]. WHO. URL https://www.who.int/foodsafety/publications/antimicrobials-sixth/en/. Accessed $16 / 02 / 2017$.

WHO. (2020). WHO | Priority pathogens and the antibiotic pipeline: an update. Bulletin of the World Health Organization, 98.

WHO, (World Health Organization). (2020b). Antibiotic resistance [Internet document] URL https://www.who.int/news-room/fact-sheets/detail/antibiotic-resistance. Accessed 23/11/2020.

Wiegand, I., Hilpert, K. \& Hancock, R.E.W. (2008). Agar and broth dilution methods to determine the minimal inhibitory concentration (MIC) of antimicrobial substances. Nature Protocols, 3, 163-175.

Winokur, P.L., Vonstein, D.L., Hoffman, L.J., Uhlenhopp, E.K. \& Doern, G.V. (2001). Evidence for Transfer of CMY-2 AmpC $\beta$-Lactamase Plasmids between Escherichia coli and Salmonella Isolates from Food Animals and Humans. Antimicrobial Agents and Chemotherapy, 45, 2716-2722.

Wintersdorff, C.J.H. von, Penders, J., Niekerk, J.M. van, Mills, N.D., Majumder, S., Alphen, L.B. van, Savelkoul, P.H.M. \& Wolffs, P.F.G. (2016). Dissemination of 
Antimicrobial Resistance in Microbial Ecosystems through Horizontal Gene Transfer. Frontiers in Microbiology, 7.

Wintersdorff, V., H, C.J., Penders, J., Niekerk, V., M, J., Mills, N.D., Majumder, S., Alphen, V., B, L., Savelkoul, P.H.M. \& Wolffs, P.F.G. (2016). Dissemination of Antimicrobial Resistance in Microbial Ecosystems through Horizontal Gene Transfer. Frontiers in Microbiology, 7.

Wong, M.H.Y., Wan, H.Y. \& Chen, S. (2013). Characterization of Multidrug-Resistant Proteus mirabilis Isolated from Chicken Carcasses. Foodborne Pathogens and Disease, 10, 177-181.

Wong, S.Y., Lee, J.S., Kwak, H.K., Via, L.E., Boshoff, H.I.M. \& Barry, C.E. (2011). Mutations in gidB Confer Low-Level Streptomycin Resistance in Mycobacterium tuberculosis. Antimicrobial Agents and Chemotherapy, 55, 2515-2522.

Wyres, K.L. \& Holt, K.E. (2018). Klebsiella pneumoniae as a key trafficker of drug resistance genes from environmental to clinically important bacteria. Current Opinion in Microbiology, Antimicrobials * Microbial systems biology, 45, 131139.

Xu, D., Nie, Q., Wang, W., Shi, L. \& Yan, H. (2016). Characterization of a transferable bcr $A B C$ and $c a d A C$ genes-harboring plasmid in Listeria monocytogenes strain isolated from food products of animal origin. International Journal of Food Microbiology, 217, 117-122.

Yachison, C.A., Yoshida, C., Robertson, J., Nash, J.H.E., Kruczkiewicz, P., Taboada, E.N., Walker, M., Reimer, A., Christianson, S., Nichani, A., Committee, T.P.C.S., Nadon, C., Paccagnella, A., Hoang, L., Chui, L., Levett, P., McDonald, R., Wylie, J., Alexander, D., Allen, V., Maki, A., Bekal, S., Davidson, R., Nickerson, E., Reid, J., Gilbert, L., German, G., Elmufti, M., Quinlan, S., Carrillo, C., Allain, R., Pagotto, F., Tschetter, L. \& Ziebell, K. (2017). The Validation and Implications of Using Whole Genome Sequencing as a Replacement for Traditional Serotyping for a National Salmonella Reference Laboratory. Frontiers in Microbiology, 8, 1044.

Yamasaki, K., Komatsu, M., Abe, N., Fukuda, S., Miyamoto, Y., Higuchi, T., Ono, T., Nishio, H., Sueyoshi, N., Kida, K., Satoh, K., Toyokawa, M., Nishi, I., Sakamoto, M., Akagi, M., Nakai, I., Kofuku, T., Orita, T., Wada, Y., Jikimoto, T., Kinoshita, S., Miyamoto, K., Hirai, I. \& Yamamoto, Y. (2010). Laboratory Surveillance for Prospective Plasmid-Mediated AmpC $\beta$-Lactamases in the Kinki Region of Japan. Journal of Clinical Microbiology, 48, 3267-3273.

Ye, J., Coulouris, G., Zaretskaya, I., Cutcutache, I., Rozen, S. \& Madden, T.L. (2012). Primer-BLAST: a tool to design target-specific primers for polymerase chain reaction. BMC bioinformatics, 13, 134. 
Yigit, H., Queenan, A.M., Rasheed, J.K., Biddle, J.W., Domenech-Sanchez, A., Alberti, S., Bush, K. \& Tenover, F.C. (2003). Carbapenem-Resistant Strain of Klebsiella oxytoca Harboring Carbapenem-Hydrolyzing $\beta$-Lactamase KPC-2. Antimicrobial Agents and Chemotherapy, 47, 3881-3889.

Yoon, K.-B., Song, B.-J., Shin, M.-Y., Lim, H.-C., Yoon, Y.-H., Jeon, D.-Y., Ha, H., Yang, S.-I. \& Kim, J.-B. (2017). Antibiotic Resistance Patterns and Serotypes of Salmonella spp. Isolated at Jeollanam-do in Korea. Osong Public Health and Research Perspectives, 8, 211-219.

Yoshida, C.E., Kruczkiewicz, P., Laing, C.R., Lingohr, E.J., Gannon, V.P.J., Nash, J.H.E. \& Taboada, E.N. (2016). The Salmonella In Silico Typing Resource (SISTR): An Open Web-Accessible Tool for Rapidly Typing and Subtyping Draft Salmonella Genome Assemblies. PLOS ONE, 11, e0147101.

Yu, G., Smith, D.K., Zhu, H., Guan, Y. \& Lam, T.T.-Y. (2017). ggtree: an r package for visualization and annotation of phylogenetic trees with their covariates and other associated data. Methods in Ecology and Evolution, 8, 28-36.

Zaheer, R., Noyes, N., Ortega Polo, R., Cook, S.R., Marinier, E., Van Domselaar, G., Belk, K.E., Morley, P.S. \& McAllister, T.A. (2018). Impact of sequencing depth on the characterization of the microbiome and resistome. Scientific Reports, 8.

Zankari, E. (2014). Comparison of the Web Tools ARG-ANNOT and ResFinder for Detection of Resistance Genes in Bacteria. Antimicrobial Agents and Chemotherapy, 58, 4986-4986.

Zankari, E., Allesøe, R., Joensen, K.G., Cavaco, L.M., Lund, O. \& Aarestrup, F.M. (2017). PointFinder: a novel web tool for WGS-based detection of antimicrobial resistance associated with chromosomal point mutations in bacterial pathogens. The Journal of Antimicrobial Chemotherapy, 72, 2764-2768.

Zankari, E., Hasman, H., Cosentino, S., Vestergaard, M., Rasmussen, S., Lund, O., Aarestrup, F.M. \& Larsen, M.V. (2012a). Identification of acquired antimicrobial resistance genes. The Journal of Antimicrobial Chemotherapy, 67, 2640-2644.

Zhan, Z., Hu, L., Jiang, X., Zeng, L., Feng, J., Wu, W., Chen, W., Yang, H., Yang, W., Gao, B., Yin, Z. \& Zhou, D. (2018). Plasmid and chromosomal integration of four novel blaIMP-carrying transposons from Pseudomonas aeruginosa, Klebsiella pneumoniae and an Enterobacter sp. The Journal of Antimicrobial Chemotherapy, 73, 3005-3015.

Zhang, S., Bakker, H.C. den, Li, S., Chen, J., Dinsmore, B.A., Lane, C., Lauer, A.C., Fields, P.I. \& Deng, X. (2019). SeqSero2: Rapid and Improved Salmonella Serotype Determination Using Whole-Genome Sequencing Data. Applied and Environmental Microbiology, 85. 
Zhang, S., Yin, Y., Jones, M.B., Zhang, Z., Kaiser, B.L.D., Dinsmore, B.A., Fitzgerald, C., Fields, P.I. \& Deng, X. (2015). Salmonella Serotype Determination Utilizing High-throughput Genome Sequencing Data. Journal of Clinical Microbiology, JCM.00323-15.

Zhang, T., Zhang, X.-X. \& Ye, L. (2011). Plasmid metagenome reveals high levels of antibiotic resistance genes and mobile genetic elements in activated sludge. PloS One, 6, e26041.

Zhao, S., McDermott, P.F., White, D.G., Qaiyumi, S., Friedman, S.L., Abbott, J.W., Glenn, A., Ayers, S.L., Post, K.W., Fales, W.H., Wilson, R.B., Reggiardo, C. \& Walker, R.D. (2007). Characterization of multidrug resistant Salmonella recovered from diseased animals. Veterinary Microbiology, 123, 122-132.

Zhao, S., Tyson, G.H., Chen, Y., Li, C., Mukherjee, S., Young, S., Lam, C., Folster, J.P., Whichard, J.M. \& McDermott, P.F. (2016). Whole-Genome Sequencing Analysis Accurately Predicts Antimicrobial Resistance Phenotypes in Campylobacter spp. Applied and Environmental Microbiology, 82, 459-466.

Zurfluh, K., Hächler, H., Nüesch-Inderbinen, M. \& Stephan, R. (2013). Characteristics of Extended-Spectrum $\beta$-Lactamase- and Carbapenemase-Producing Enterobacteriaceae Isolates from Rivers and Lakes in Switzerland. Applied and Environmental Microbiology, 79, 3021-3026. 